Ph.D. Thesis

\title{
RIDER - A RECOMMENDATION FRAMEWORK FOR EXPLOITING EVALUATION RESULTS AND UsER QUALITY REQUIREMENTS
}

Author: Filip Radulović

Supervisor: Dr. Raúl García Castro

Supervisor: Prof. Dr. Asunción Gómez Pérez

June 2016 

Tribunal nombrado por el Sr. Rector Magfco. de la Universidad Politécnica de Madrid, el día 12 de mayo de 2016.

Presidente: Dr. Oscar Corcho

Vocal: $\quad$ Dr. Antonio Bahamonde Rionda

Vocal: Dr. Jérôme Euzenat

Vocal: $\quad$ Dr. Milan Stanković

Secretario: Dr. Jacinto González-Pachón

Suplente: Dr. Mariano Fernández López

Suplente: Dr. Adolfo Lozano-Tello

Realizado el acto de defensa y lectura de la Tesis el día 27 de junio de 2016 en la Escuela Técnica Superior de Ingenieros Informáticos

Calificación:

EL PRESIDENTE

VOCAL 1

VOCAL 2

VOCAL 3

EL SECRETARIO 

To measure is to know.

Lord Kelvin 



\section{Acknowledgements}

A long-awaited moment in life for every Ph.D. student is the one when the time comes to write the last page of his or her thesis: this page. But one should not be easily confused; this last written page is actually the beginning of it all.

This work is the result of a lot of devotion, hard work and sweat. And renunciation, but not only by its author. I wish to thank to my family, my parents Dragana and Momčilo and my brother Marko. For their support and all the moments missed, good ones and bad ones.

I owe my extensive gratitude to Raúl and Asun, my supervisors on this long but fruitful journey, for making all of this possible and for guiding me through such an important part of my professional life. For the time and effort devoted, patience and, above all, their trust in me and this work.

I owe my special gratitude to Jelena and Milan, who ignited an initial spark in my love and devotion to research. For being an inspiration and teaching me to always strive for excellence.

Finally, I wish to mention a number of great people who, in one way or another, helped me and were part of this work. I have to thank to my OEG colleagues, María, Esther, Idafen and Dani Garijo for being kind and friendly to me from my first day in the group. Also, many thanks to Miguel Angel, Andres, Olga, Pablo, Julia, Carlos Badenes, Freddy, Nandana, Dani Vila, Mari-Carmen, Elena, Victor, Jorge, Ahmad, Fran, Alejandro Llaves, Almudena, Pierre, and Carlos Buil. I also have to mention people who, at some point in time, were visitors in the group, Jose, Rosangelis, Adrian Lopez, and Deisy. Thanks to Laura and Fric. Last, but certainly not least, I owe my thanks to Nikola, Dragan, and Vladan.

The work in this thesis has been supported by the FPU grant (FPU2012/04084) of the Spanish Ministry of Education, Culture and Sport.

In Madrid, $13^{\text {th }}$ of March 2016. 



\section{Abstract}

Analytic Network Process and Analytic Hierarchy Process are Multiple Criteria Decision Making methods which are often used for providing recommendations in terms of rankings in order to help with the problem of selecting one product, service or action among a group of possible alternatives. The usage of the Analytic Network Process and of the Analytic Hierarchy Process implies a definition of a model of a recommendation, and involvement of persons (users or experts in the field related to the alternatives to be recommended) for the manual comparison of alternatives according to their quality by specifying preferences (importance) of one alternative over another.

However, different users have different quality requirements over alternatives and every specific set of quality requirements implies repeated definition of a model of a recommendation adapted to that set of quality requirements, which is a resource consuming task. Furthermore, relying on user or expert comparison of alternatives is often expensive since it requires knowledge about all the alternatives and about the comparison method used with the Analytic Hierarchy or Network Process. On the other hand, in some fields in which the selection of the best alternative is a difficult task, such as the field of Semantic Web and semantic technologies, there exist a number of alternatives' quality evaluation results that can be used for the comparison of alternatives and deriving preferences between these alternatives. Such evaluation results are usually obtained in the process of evaluation driven by the guidelines defined in a quality model.

This thesis aims to advance the current state of the art in the Multiple Criteria Decision Making field and in the semantic technologies field. In particular, the main goals of this thesis are: i) to provide a Multiple Criteria Decision Making framework that extends the Analytic Network Process and that takes advantage of evaluation results of alternatives and of user quality requirements over such alternatives; and ii) to apply this MCDM framework in the Semantic Web field for semantic tools recommendation. To achieve these goals, the following contributions are delivered in this thesis:

- A set of domain-independent algorithms for the automatic comparison of 
alternatives according to alternatives' evaluation results, which can be used in the Analytic Network Process and in the Analytic Hierarchy Process.

- A set of methods for the dynamic extraction of the Analytic Network Process and Analytic Hierarchy Process models, that are based on user quality requirements.

- Software that supports the proposed MCDM framework.

- SemQuaRE, a quality model for semantic tools.

- The application of the proposed MCDM framework for the recommendation of semantic technologies, including a web application for the recommendation of such technologies.

The MCDM framework proposed in this thesis is generic and can be instantiated in any domain by defining a quality model, an AHP or ANP model of the domain, criteria pairwise comparisons (as required by the AHP or the ANP) made by experts, and taking evaluation results of alternatives as inputs. Once instantiated, the proposed framework takes a set of quality requirements specified by a user as input and produces as output a ranked list of alternatives that best suit the specified user quality requirements. 


\section{Resumen}

El Proceso Analítico en Red y el Proceso Analítico Jerárquico son métodos de toma de decisiones multicriterio que a menudo se utilizan para proporcionar recomendaciones (en términos de un ranking) con el fin de ayudar en el problema de selección de un producto, servicio o acción entre un grupo de posibles alternativas. El uso del Proceso Analítico en Red y del Proceso Analítico Jerárquico implica la definición de un modelo de recomendación y la participación de personas (usuarios o expertos en el ámbito relacionado con las alternativas a ser recomendadas) en la comparación manual de alternativas de acuerdo a su calidad mediante la especificación de preferencias (importancias) de una alternativa frente a otra.

Sin embargo, distintos usuarios tienen diferentes requisitos de calidad para dichas alternativas y cada conjunto de requisitos de calidad específico implica la repetición de la definición del modelo de recomendación adaptado a ese conjunto de requisitos de calidad, que es una tarea que consume muchos recursos. Por otra parte, basar la comparación de alternativas en el usuario o experto es a menudo costoso, ya que requiere conocimientos sobre todas las alternativas y sobre el método de comparación utilizado en el Proceso Analítico Jerárquico o en el Proceso Analítico en Red. Por otra parte, en algunos campos en los que la selección de la mejor alternativa es una tarea difícil, como el campo de la Web Semántica y de las tecnologías semánticas, existen una serie de resultados de la evaluación de calidad de alternativas que pueden ser utilizados para la comparación y derivación de preferencias entre estas alternativas. Tales resultados de evaluación normalmente se obtienen mediante un proceso de evaluación impulsado por las guías definidas en un modelo de calidad.

Esta tesis tiene como objetivo avanzar el estado del arte en el campo de métodos de toma de decisiones multicriterio y en el campo de las tecnologías semánticas. En particular, los principales objetivos de esta tesis son: i) proporcionar un marco de recomendación basado en un método de toma de decisiones multicriterio que extiende el Proceso Analítico en Red y que se aprovecha de los resultados de evaluación de alternativas y de los requisitos de calidad de los usuarios relacionados con estas alternativas; y ii) aplicar este marco de recomendación en el campo de la Web Semántica para la recomendación de tecnologías semánticas. Para alcanzar estos objetivos, las siguientes contribuciones se presentan en esta tesis: 
- Un conjunto de los algoritmos independientes de dominio para la comparación automática de alternativas en función de sus resultados de evaluación, que se pueden utilizar en el Proceso Analítico en Red y en el Proceso Analítico Jerárquico.

- Un conjunto de métodos para la extracción dinámica de los modelos del Proceso Analítico en Red y del Proceso Analítico Jerárquico, que se basan en los requisitos de calidad del usuario.

- El software que soporta el marco de recomendación propuesto.

- SemQuaRE, un modelo de calidad para las tecnologías semánticas.

- La aplicación del propuesto marco de recomendación para la recomendación de tecnologías semánticas, incluyendo una aplicación web para la recomendación de este tipo de tecnologías.

El marco de recomendación propuesto en esta tesis es genérico y puede ser instanciado en cualquier dominio mediante la definición de un modelo de calidad, un modelo de AHP o ANP del dominio, la comparación de los criterios (según los requisitos del AHP o el ANP) hecha por los expertos, y tomando como entrada los resultados de la evaluación de alternativas. Una vez instanciado, el marco propuesto toma como entrada un conjunto de requisitos de calidad especificados por un usuario y produce como salida un ranking de las alternativas que mejor se adapten a dichos requisitos de calidad. 


\section{Contents}

Acknowledgements $\quad$ i

Abstract $\quad$ iii

Resumen $\quad$ v

List of Figures xiii

List of Tables $\quad$ xvii

List of Algorithms $\quad$ xxi

1 Introduction $\mathbf{1}$

1.1 Multiple Criteria Decision Making . . . . . . . . . . . . . . . 1

1.1.1 Multiple Criteria Decision Making Methods . . . . . . . . . . 2

1.1.2 Quality Models in Multiple Criteria Decision Making . . . . . 3

1.1.3 Need for Exploiting Evaluation Results and User Quality Re-

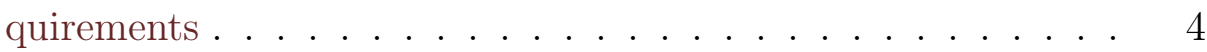

1.2 Semantic Technology Recommendation . . . . . . . . . . . . . . 5

1.2.1 Semantic Technologies . . . . . . . . . . . . . 5

1.2.2 The Need for Semantic Technology Recommendation . . . . . 6

1.2.3 The Need for a Semantic Technology Quality Model . . . . . . 7

1.3 Main Contributions . . . . . . . . . . . . . . . . . . 7

1.4 Structure of the Document . . . . . . . . . . . . . . . . 8

1.5 Derived Publications . . . . . . . . . . . . . . . . . 9 9

1.6 Research Stays . . . . . . . . . . . . . . . . . . . . . . 11

1.7 Work in Research and Innovation Projects . . . . . . . . . . . . . 11

2 State of the Art $\quad 13$

2.1 Multiple Criteria Decision Making . . . . . . . . . . . . . . . . . 13

2.1.1 General MCDM Recommendation Framework . . . . . . . . . 15 
$2.1 .2 \quad$ ELECTRE . . . . . . . . . . . . . . . . . . . 15

2.1 .3 PROMETHEE . . . . . . . . . . . . . . . . . 16

2.1.4 Analytic Hierarchy Process . . . . . . . . . . . . . . . . . . . . 19

2.1.5 Analytic Network Process . . . . . . . . . . . . . . . 23

2.1.6 Applications of Multiple Criteria Decision Making Methods in the Software Engineering Field _ . . . . . . . . . . . 26

2.2 Software Quality Models . . . . . . . . . . . . . . . . . . 34

2.2 .1 McCall's Model . . . . . . . . . . . . . . . . . . . . . 35

2.2 .2 Boehm's Model . . . . . . . . . . . . . . . . . . . . . 35

2.2 .3 ISO 9126's Model . . . . . . . . . . . . . . . . . . . . . . . 36

2.2 .4 SQuaRE's Model . . . . . . . . . . . . . . . . . . . 37

2.2.5 Quality Model Terminology . . . . . . . . . . . . . . . . 39

2.2.6 Approaches for Extending Software Quality Models . . . . . . 39

2.3 Semantic Technology Quality . . . . . . . . . . . . . . . 42

2.3.1 Semantic Technology Quality Specification . . . . . . . . . . 42

2.3.2 Semantic Technology Evaluation Results . . . . . . . . . . . . 47

2.4 Semantic Technology Recommendation . . . . . . . . . . . . 49

2.5 Conclusions to the State of the Art . . . . . . . . . . . . 50

3 Work Objectives

3.1 Thesis Objectives and Open Research Problems . . . . . . . . . 53

3.2 Contributions to the State of the Art . . . . . . . . . . . . 55

3.3 Work Assumptions, Hypotheses, and Restrictions . . . . . . . . 57

3.4 Evaluation $\operatorname{Plan} \ldots \ldots \ldots \ldots \ldots$

3.5 Research Methodology . . . . . . . . . . . . . . . . . . . . 62

3.5.1 Description of the Research Process . . . . . . . . . . . . . 64

4 The RIDER Recommendation Framework $\quad 67$

4.1 Quality Model . . . . . . . . . . . . . . . . . . 68

4.1.1 Quality Model Ontology . . . . . . . . . . . . . 72

4.2 Evaluation Results of Alternatives . . . . . . . . . . . . . . . 74

4.2.1 Evaluation Result Ontology . . . . . . . . . . . . . 75

4.3 Comparison Algorithm . . . . . . . . . . . . . . . . 77

4.4 User Quality Requirements . . . . . . . . . . . . . . . . . . . 85

4.5 Model Extraction Methods . . . . . . . . . . . . . . . . . . 93

4.6 Criteria Pairwise Comparisons . . . . . . . . . . . . . . . . . 98

4.7 Instantiation of the RIDER Recommendation Framework . . . . . . . 102

4.8 RIDER Software Support _. . . . . . . . . . . . . . . . . 104

4.8.1 The jRIDER Java Library . . . . . . . . . . . . . . . . 104

4.8.2 The RIDER Web Application . . . . . . . . . . . . . . 106

4.9 Summary . . . . . . . . . . . . . . . . . . . . . . . . 109 
5 SemQuaRE - A Quality Model for Semantic Technologies 111

5.1 Bottom-up Method for Extending Software Quality Models . . . . . . 112

5.2 Identifying Base Measures . . . . . . . . . . . . . . . . . 115

5.3 Identifying Derived Measures . . . . . . . . . . . . . . . . . . 118

5.4 Identifying Indicators . . . . . . . . . . . . . . . . . . . . . . . . . . . 119

5.5 Specifying Relationships Between Measures . . . . . . . . . . . . 120

5.6 Defining Domain-Specific Quality Sub-characteristics . . . . . . . . . 123

5.7 Aligning Quality Sub-Characteristics with a Quality Model . . . . . . 127

5.8 Summary . . . . . . . . . . . . . . . . 127

6 RIDER for Semantic Technologies 131

6.1 Semantic Technology Evaluation Results . . . . . . . . . . . . . . 131

6.2 ANP Network for Semantic Technologies . . . . . . . . . . . . . 136

6.3 Pairwise Comparisons of Criteria . . . . . . . . . . . . . 139

6.4 Using RIDER for Semantic Technology Recommendation . . . . . . . 144

6.5 Semantic Technology Recommendation System . . . . . . . . . . . . . 148

6.6 Summary . . . . . . . . . . . . . . . . . . 151

7 Evaluation $\quad 153$

7.1 Evaluation of the Comparison Algorithm without Thresholds . . . . . 153

7.1.1 Evaluation Datasets . . . . . . . . . . . . . . . . 155

7.1.2 Uniform Dataset without Thresholds . . . . . . . . . . . . . . 157

7.1.3 Normal Dataset without Thresholds . . . . . . . . . . . . . . . 159

7.1.4 Erlang Dataset without Thresholds . . . . . . . . . . . . . . . 159

7.1.5 Discussion on the Evaluation without Thresholds . . . . . . . 159

7.2 Evaluation of the Comparison Algorithm with Thresholds . . . . . . . 161

7.2.1 Uniform Dataset with Thresholds . . . . . . . . . . . . . . . . 162

7.2.2 Normal Dataset with Thresholds . . . . . . . . . . . . . . 164

7.2.3 Erlang Dataset with Thresholds . . . . . . . . . . . . . . . . 165

7.2.4 Discussion on the Evaluation with Thresholds . . . . . . . . . 165

7.3 Evaluation of the Model Extraction Methods . . . . . . . . . . . . . 166

7.3.1 Discussion on the Model Extraction Methods Evaluation . . . 171

7.4 Evaluation of the SemQuaRE Quality Model . . . . . . . . . . . . . . 172

7.4.1 Representativeness . . . . . . . . . . . . . . . 172

7.4 .2 Flexibility ... . . . . . . . . . . . . . . . 173

7.4.3 Discussion on the SemQuaRE Quality Model Evaluation . . . 174

7.5 Evaluation of the RIDER Framework with User Feedback . . . . . . . 177

7.5.1 Setup of the Evaluation with User Feedback . . . . . . . . . . 179

7.5.2 Results of the Evaluation with User Feedback . . . . . . . . . 180

7.5.3 Discussion on the Evaluation with User Feedback . . . . . . . 182 
Contents

8 Conclusions and Future Work

A List of Publications Analysed in the Semantic Technology Quality Specification Review 205

B Detailed Description of the SemQuaRE Quality Model 217

B.1 Ontology Engineering Tools . . . . . . . . . . . . . 217

B.1.1 Test Data . . . . . . . . . . . . . . . . 217

B.1.2 Base Measures . . . . . . . . . . . . . . . . . . 219

B.1.3 Derived Measures . . . . . . . . . . . . . . . . . 219

B.1.4 Ontology Engineering Tools Indicators . . . . . . . . . . . . . 223

B.1.5 SquaRE Quality Characteristics . . . . . . . . . . . . 225

B.1.6 Ontology Engineering Tools Quality Characteristics . . . . . . 225

B.2 Reasoning Systems . . . . . . . . . . . . . . . . . . 227

B.2.1 Test Data . . . . . . . . . . . . . . . . . . 227

B.2.2 Base Measures . . . . . . . . . . . . . . . . . . 230

B.2.3 Reasoning Systems Indicators . . . . . . . . . . . . . . . . 232

B.2.4 SQuaRE Quality Characteristics . . . . . . . . . . . . . 236

B.2.5 Reasoning Systems Quality Characteristics . . . . . . . . . . 236

B.3 Ontology Matching Tools . . . . . . . . . . . . . . . 237

B.3.1 Test Data . . . . . . . . . . . . . . . . . . . . 239

B.3.2 Base Measures . . . . . . . . . . . . . . . . 239

B.3.3 Derived Measures . . . . . . . . . . . . . . . . . . 239

B.3.4 Ontology Matching Tools Indicators . . . . . . . . . . . . . . . 240

B.3.5 SQuaRE Quality Characteristics . . . . . . . . . . . . 241

B.3.6 Ontology Matching Tools Quality Characteristics . . . . . . . 241

B.4 Semantic Search Tools . . . . . . . . . . . . . . . . . . 243

B.4.1 Test Data . . . . . . . . . . . . . . . . . . 245

B.4.2 Base Measures . . . . . . . . . . . . . . . . . . 245

B.4.3 Derived Measures . . . . . . . . . . . . . . . . 246

B.4.4 Semantic Search Tools Indicators . . . . . . . . . . . . . . 248

B.4.5 SQuaRE Quality Characteristics . . . . . . . . . . . . . . . 251

B.4.6 Semantic Search Tools Quality Characteristics . . . . . . . . . 252

B.5 Semantic Web Service Tools . . . . . . . . . . . . . . . 253

B.5.1 Test Data . . . . . . . . . . . . . . . . . . 253

B.5.2 Base Measures . . . . . . . . . . . . . . . 254

B.5.3 Derived Measures . . . . . . . . . . . . . . 255

B.5.4 Semantic Web Service Tools Indicators . . . . . . . . . . . . . 256

B.5.5 SQuaRE Quality Characteristics . . . . . . . . . . . . 257 
B.5.6 Semantic Web Service Tools Quality Characteristics . . . . . . 257

B.6 Ontology Annotation Tools . . . . . . . . . . . . . . . 258

B.6.1 Test Data . . . . . . . . . . . . . . . . . . . . 258

B.6.2 Base Measures . . . . . . . . . . . . . . . . . 260

B.6.3 Derived Measures . . . . . . . . . . . . . . . . . . . 260

B.6.4 Ontology Annotation Tools Indicators . . . . . . . . . . . . . 261

B.6.5 SQuaRE Quality Characteristics . . . . . . . . . . . . . 261

B.6.6 Ontology Annotation Tools Quality Characteristics . . . . . . 262

B.7 Complete Overview of the Quality Model . . . . . . . . . . . . . 262 



\section{List of Figures}

2.1 General MCDM recommendation framework. . . . . . . . . . . . . 15

2.2 Six types of preference functions in PROMETHEE methods [15]. $p$ and $q$ are thresholds of indifference and strict preference, respectively. 18

2.3 The hierarchical model of the AHP. . . . . . . . . . . . . . 21

2.4 ANP workflow. . . . . . . . . . . . . . . . . . . . . . 24

2.5 An example of an ANP network, with criteria $\left(\mathrm{C}_{i j}\right)$ and alternatives $\left(\mathrm{A}_{k}\right)$ as elements that are grouped into clusters $\left(\mathrm{CL}_{i}\right)$ and the dependencies between them. . . . . . . . . . . . . . . . 25

2.6 Recommendation framework based on the AHP/ANP. . . . . . . . . 27

2.7 ISO 9126 internal and external quality characteristics and sub-characteristics [61]. . . . . . . . . . . . . . . . . . 36

2.8 SQuaRE quality model - Product quality [65]. . . . . . . . . . . . . . 38

2.9 SQuaRE quality model - Quality in use [65]. . . . . . . . . . . . . . . 38

2.10 Quality model elements. . . . . . . . . . . . . . . . . 39

3.1 Mapping between the objectives, contributions, assumptions, hypotheses, and restrictions. . . . . . . . . . . . . . . 60

3.2 Inputs taken into account for delivering thesis' contributions. . . . . . 62

3.3 Phases of the thesis development. . . . . . . . . . . . 65

4.1 Overview of the RIDER framework. . . . . . . . . . . . . . . . 69

4.2 RIDER framework with the quality models component. . . . . . . . . 70

4.3 Sample subset of the semantic technology ANP network. . . . . . . . 72

4.4 Graphical representation of the Quality Model ontology. . . . . . . . . 73

4.5 RIDER framework with the evaluation results component. . . . . . . 75

4.6 Graphical representation of the Evaluation Result ontology. . . . . . . 76

4.7 RIDER framework with the comparison algorithm component. . . . . 78

4.8 Inputs and outputs of the comparison algorithm without threshold. . 79

4.9 Scale distance algorithm visualization. . . . . . . . . . . . . . . . . . . 82

4.10 Maximum distance algorithm visualization. . . . . . . . . . . . . . . 84

4.11 RIDER framework with the user quality requirements component. . . 86 
4.12 Inputs and outputs of the comparison algorithm with threshold. . . . 86

4.13 RIDER framework with the model extraction method component. . . 94

4.14 An example of the ANP network of a domain. . . . . . . . . . . . . 97

4.15 The ANP network after the network extraction process. . . . . . . . . 97

4.16 RIDER framework with criteria pairwise comparison component. . . . 98

4.17 ANP/AHP and RIDER use case diagram. . . . . . . . . . . . . . . . 103

4.18 jRIDER architecture. . . . . . . . . . . . . . . . . 105

4.19 Architecture of the RIDER web application. . . . . . . . . . . . . 107

5.1 Structure of the quality model for semantic technologies. . . . . . . . 112

5.2 The method for extending software quality models based on a bottomup approach. . . . . . . . . . . . . . . . . 113

5.3 Conformance test execution of ontology engineering tools [38]. . . . . 116

5.4 Accuracy test execution of ontology matching tools. . . . . . . . . . . 117

5.5 Entities in the conformance scenario of ontology engineering tools. . . 124

5.6 Entities in the accuracy scenario of ontology matching tools. . . . . . 125

5.7 Quality characteristics and sub-characteristics for semantic technologies defined based on the SEALS evaluation results. . . . . . . . . . . . . 129

6.1 Calculation of quality indicators from the SEALS evaluation results. . 132

6.2 Semantic technology ANP network. . . . . . . . . . . . . . 138

6.3 The ANP network for the ontology matching tools use case. . . . . . 145

6.4 The first page of the semantic technology recommendation system. . . 149

6.5 Results for the recommendation. . . . . . . . . . . . . . . . . 150

6.6 Results for all the tools with respect to the quality requirements. . . . 150

6.7 Results for a particular tool. . . . . . . . . . . . . . . . 151

6.8 Part of the weighted supermatrix related to quality indicators (criteria). 151

7.1 Evaluation of the comparison algorithm without thresholds. . . . . . 154

7.2 Evaluation datasets. . . . . . . . . . . . . . . . . 156

7.3 Comparisons with respect to result distances for different datasets without threshold. . . . . . . . . . . . . . . . . . . 158

7.4 Evaluation of the comparison algorithm with thresholds. . . . . . . . 162

7.5 Comparisons with respect to result distances for different datasets with threshold. . . . . . . . . . . . . . . . . . . 163

7.6 Evaluation of the model extraction methods. . . . . . . . . . . 167

7.7 ANP network used in the evaluation. . . . . . . . . . . . . . 168

7.8 ANP network for the first quality requirements example obtained using the Requirements network and the Partial network extraction methods.170

7.9 Quality characteristics and sub-characteristics for semantic technologies upon completing the evaluation process. . . . . . . . . . . 176 
7.10 Evaluation of RIDER framework with user feedback. . . . . . . . . . 178

B.1 Conformance scenario of ontology engineering tools. . . . . . . . . . 218

B.2 Interoperability scenario of ontology engineering tools. . . . . . . . . 218

B.3 Scalability scenario of ontology engineering tools. . . . . . . . . . . 218

B.4 Quality characteristics of ontology engineering tools. . . . . . . . . . 227

B.5 Classification scenario of reasoning systems. . . . . . . . . . . . . 228

B.6 Class satisfiability scenario of reasoning systems. . . . . . . . . . . . . 228

B.7 Ontology satisfiability scenario of reasoning systems. . . . . . . . . 229

B.8 Entailment scenario of reasoning systems. . . . . . . . . . . . . . 229

B.9 Non-entailment scenario of reasoning systems. . . . . . . . . . . . 230

B.10 Quality characteristics of reasoning systems. . . . . . . . . . . . 238

B.11 Accuracy scenario of ontology matching tools. . . . . . . . . . . . 238

B.12 Quality characteristics of ontology matching tools. . . . . . . . . . . . 242

B.13 Automated scenario (I) of semantic search tools. . . . . . . . . . . . . 243

B.14 Automated scenario (II) of semantic search tools. . . . . . . . . . . 243

B.15 User-in-the-loop scenario (I) of semantic search tools. . . . . . . . . . 244

B.16 User-in-the-loop scenario (II) of semantic search tools. . . . . . . . . . 244

B.17 Quality characteristics of semantic search tools. . . . . . . . . . 253

B.18 Accuracy scenario of semantic web service tools. . . . . . . . . . . . 254

B.19 Quality characteristics of semantic web service tools. . . . . . . . 259

B.20 Accuracy scenario of ontology annotation tools. . . . . . . . . . . . 259

B.21 Quality characteristics of ontology annotation tools. . . . . . . . . . . 262

B.22 Quality characteristics of semantic technologies. . . . . . . . . . 263 



\section{List of Tables}

2.1 The AHP fundamental scale [118]. . . . . . . . . . . . . . 20

2.2 Example of the pairwise comparison in the AHP. . . . . . . . 20

2.3 Random index [118]. . . . . . . . . . . . . . . . . . . . 22

2.4 Example of an ANP supermatrix, related to the network on Figure 2.5. 25

2.5 Example of an ANP cluster matrix, related to the network on Figure 2.5. 26

2.6 Use of MCDM methods in software engineering (ELECTRE, PROMETHEE, and $\mathrm{ANP}) \ldots \ldots \ldots \ldots \ldots \ldots \ldots$

2.7 Use of MCDM methods in software engineering (AHP) . . . . . . . 31

2.8 Elements of the described quality models. . . . . . . . . . . . 34

2.9 ISO 9126 internal and external measures for the Functionality quality

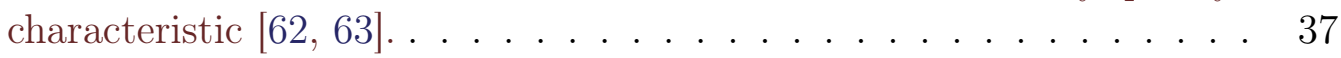

2.10 Quality model terminology. . . . . . . . . . . . . . . . 40

2.11 Characteristics and measures used in conference publications for ontology engineering tools. . . . . . . . . . . . . . . . 45

2.12 Characteristics and measures used in conference publications for ontology matching tools. . . . . . . . . . . . . . . . 46

2.13 Characteristics and measures used in conference publications for reasoning systems. . . . . . . . . . . . . . . 46

2.14 Characteristics and measures used in conference publications for semantic search tools. . . . . . . . . . . . . . . . . 46

2.15 Characteristics and measures used in conference publications for semantic web services. . . . . . . . . . . . . . . . 46

2.16 Measures used in the first and the second SEALS evaluation campaigns. 49

4.1 Comparison algorithms depending on the type of scale and their inputs. 79

4.2 An example of alternatives comparison using the Maximum distance algorithm. . . . . . . . . . . . . . . . . 84

4.3 An example of alternatives comparison using the Maximum distance algorithm with threshold. . . . . . . . . . . . . . . . . . 92

4.4 An example of criteria pairwise comparison with respect to Average alignment F-measure ${ }^{1}$. . . . . . . . . . . . . . . . . . . . . 99 
5.1 Number of measures obtained for semantic technologies based on the SEALS evaluation results. . . . . . . . . . . . . . . . . . . 119

5.2 Semantic quality sub-characteristics and indicators defined based on the SEALS evaluation results. . . . . . . . . . . . . . . . 128

6.1 Quality indicators calculated from the SEALS evaluation results. . . . 133

6.2 Tools and quality indicators for ontology engineering tools [43] . . . . 134

6.3 Tools and quality indicators for reasoning systems [142]. . . . . . . . 135

6.4 Tools and quality indicators for ontology matching tools $[88]^{2}$. . . . 135

6.5 Tools and quality indicators for semantic search tools [138] . . . . . . 136

6.6 Tools and quality indicators for semantic web service tools [78]. . . . 136

6.7 Pairwise comparisons of indicators with respect to Average alignment F-measure . . . . . . . . . . . . . . . . . . . . . . . 139

6.8 Pairwise comparisons of indicators with respect to Average alignment H-measure ${ }^{3}$. . . . . . . . . . . . . . . . . . . . . . . . . . . . 140

6.9 Pairwise comparisons of measures with respect to Average search F-measure ${ }^{4}$. . . . . . . . . . . . . . . . . . . . . 140

6.10 Pairwise comparisons of measures with respect to Entailment correct-

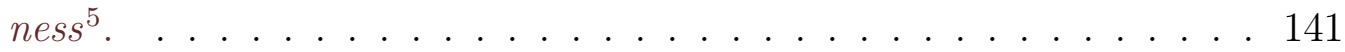

6.11 Pairwise comparisons of clusters with respect to Ontology language model interoperability ${ }^{6}$. . . . . . . . . . . . . . . . . . . . 141

6.12 Pairwise comparisons of clusters with respect to Ontology interchange

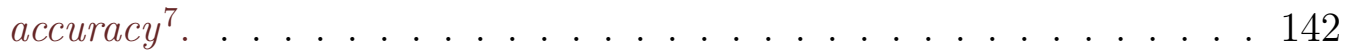

6.13 Pairwise comparisons of clusters with respect to Semantic search time behavior $^{8}$. . . . . . . . . . . . . . . . . . . . 142

6.14 Pairwise comparisons of measures with respect to Ontology processing maturity ${ }^{9}$. . . . . . . . . . . . . . . . . . . . . 142

6.15 Pairwise comparisons of clusters with respect to Reasoning accuracy ${ }^{10} .143$

6.16 Pairwise comparisons of measures with respect to Ontology interchange

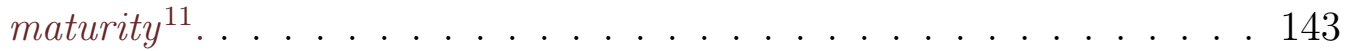

6.17 Pairwise comparisons of clusters with respect to Reasoning maturity ${ }^{12} .143$

6.18 Evaluation results for the ontology matching tools use case. . . . . . . 144

6.19 Supermatrix structure for the ontology matching tools use case ${ }^{13}$. . . 145

6.20 Comparison of the alternatives with respect to Average alignment precision using the Maximum distance algorithm with threshold. . . . 146

6.21 Comparison of the alternatives with respect to Ontology alignment errors using the Maximum distance algorithm with threshold. . . . . 146

6.22 Supermatrix for the ontology matching tools use case filled with example alternatives comparison. . . . . . . . . . . . . . 147 
6.23 Supermatrix for the ontology matching tools use case filled with criteria dependencies. . . . . . . . . . . . . . . . . . 147

6.24 Cluster matrix for the ontology matching tools use case ${ }^{14}$. . . . . . 148

6.25 Limit supermatrix for the ontology matching tools use case. . . . . . 148

7.1 Quality metrics for the different datasets without threshold. . . . . . 157

7.2 Quality metrics for the different datasets with threshold. . . . . . . . 164

7.3 Evaluation results used in the evaluation. . . . . . . . . . . . . . 168

7.4 User quality requirements and number of satisfying quality requirements. 169

7.5 Evaluation of network extraction methods for the Maximum distance algorithm. . . . . . . . . . . . . . . . . . . . . 169

7.6 Semantic quality sub-characteristics added to SemQuaRE during the flexibility evaluation. . . . . . . . . . . . . . . . 175

7.7 Evaluation results used in the evaluation with user feedback. . . . . . 180

7.8 Quality requirements and RIDER rankings for each use case. . . . . . 180

7.9 Rankings obtained by RIDER and by the users. . . . . . . . . . . . . 181 



\section{List of Algorithms}

1 General algorithm for filling a pairwise comparison matrix without threshold . . . . . . . . . . . . . . . . . . . . . . 79

2 Nominal scale algorithm . . . . . . . . . . . . . . . . 80

3 Simple algorithm . . . . . . . . . . . . . . . . . . . 81

4 Scale distance algorithm . . . . . . . . . . . . . . . . . . . . . . . . . . 82

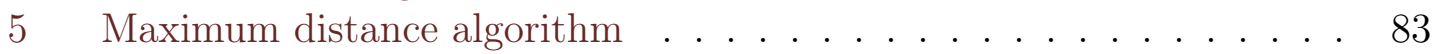

6 General algorithm for filling a pairwise comparison matrix with threshold 87

7 Nominal scale algorithm with threshold . . . . . . . . . . . . . . 88

8 Simple algorithm with threshold . . . . . . . . . . . . . . . . 89

9 Scale distance algorithm with threshold . . . . . . . . . . . . . 90

10 Maximum distance algorithm with threshold . . . . . . . . . . . . . . 91

11 Requirements hierarchy . . . . . . . . . . . . . . . . . . 95

12 Complete hierarchy . . . . . . . . . . . . . . . . . . . . . . . . . . . . . . . . . . . 95

13 Requirements network . . . . . . . . . . . . . . . . 95

14 Partial network . . . . . . . . . . . . . . . . . . 96

15 Complete network . . . . . . . . . . . . . . 96 



\section{Chapter 1}

\section{Introduction}

In their private and professional lives people often face a decision making situation in which they have to select one product, service, or action among a group of possible alternatives. They need to select which car to buy, which software to use, which doctor to see, or which investment to make.

In a decision making situation, selecting the best alternative can be a difficult task due to the uncertainty of alternative's quality and to the users' lack of experience and expertise. Because of this, decisions on the selection of the most suitable alternative in many fields require either advise from experts for assessing these alternatives or using techniques that can provide recommendations. Furthermore, expert engagement and a thorough analysis of alternatives is needed in many cases in which the selection of wrong alternatives can have a negative impact upon the usage of such wrongly selected alternatives.

An example of a field in which selecting an appropriate alternative is not an easy task and is regarded as a very important problem is the software engineering field [67]. For example, it is a well known fact that the selection of Enterprise Resource Planning (ERP) systems is a highly sophisticated process [52] which requires deep analysis of the available software and where a wrong decision can lead not just to negative influences on a company business, but also to a significant financial loss and failed investment.

\subsection{Multiple Criteria Decision Making}

Multiple Criteria Decision Making (MCDM) is a field that deals with those situations in which people need to select the right alternative that they need to use in order to solve a certain problem. In such situations, alternatives represent concrete products, services or actions that will help users in solving a specific problem and that can be assessed and compared according to different conflicting criteria, while criteria 
Chapter 1. Introduction

represent the characteristics of these alternatives that are important for making a decision.

\subsubsection{Multiple Criteria Decision Making Methods}

With the goal of making better decisions when selecting the right alternative, Multiple Criteria Decision Making methods are used. Such methods are designed to provide recommendations in terms of a ranking of alternatives from best to worse or in terms of choosing one or several best alternatives, and have been successfully applied across different fields and decision making situations.

A large number of MCDM methods has been developed to this date, which can be classified according to the three main MCDM approaches described in the literature [34, 49, 134]: outranking, single synthesizing criterion, and interactive. However, in spite of the number of different methods, no method is considered to be the best to be applied in every decision making situation [49, 126].

Among the well known and widely used MCDM methods are the Analytic Hierarchy Process [113] (AHP) and its generalization, the Analytic Network Process [114] (ANP), which is specially designed to take into account dependencies and feedbacks in the decision process [116]. The scope of this thesis lies within the ANP and the AHP MCDM methods.

Regarded as one of the main strengths [69] of the ANP and the AHP methods is that they require a formalization of a model that consists of a set of alternatives and of a set of often conflicting criteria related to the quality and performance of such alternatives, and which help in better structuring the recommendation problem. For providing recommendations in terms of alternatives ranking, the AHP and the ANP rely on comparisons of alternatives according to a set of criteria defined in a model. In these comparisons, preferences (importances) of one alternative over another alternative are defined with respect to a set of criteria.

The key concepts in both the AHP and the ANP are the use of a pairwise comparison matrix and of Saaty's ${ }^{1}$ fundamental comparison scale, which provide the possibility of measuring both tangible and intangible criteria; these two concepts together provide a mechanism for the comparison of alternatives in which alternatives are compared with respect to a certain criterion by specifying preferences (importances) of one alternative over another alternative in order to rank the alternatives with respect to that criterion. Furthermore, as both methods require the weights (importances) of the criteria to be specified, the pairwise comparison technique is also used for weight calculations.

The header elements in the rows and columns of a pairwise comparison matrix are identical, and they represent alternatives that are compared with respect to a criterion,

\footnotetext{
${ }^{1}$ Thomas L. Saaty, the developer of the AHP and the ANP.
} 
or criteria for which the weights are calculated. Each element in a pairwise comparison matrix is filled with a value from the fundamental scale [118] by answering a question "how much the alternative/criterion in the corresponding row of the observed element is better/more important/preferred than the alternative/criterion in the corresponding column" [118]. The importance of the alternatives/criteria can then be determined by calculating the average values in each row, after the matrix is normalized.

One of the advantages of pairwise comparison matrices is that they provide a method for the calculation of the consistency of comparisons, which is regarded as one of the main advantages of the AHP [55]. The matrix consistency is denoted with a consistency ratio, which is the ratio of the consistency index of a matrix and a random consistency index. In those situations where the consistency ratio is lower than 0.1 , the pairwise comparison is considered to be consistent [118]. However, in real world scenarios it can be expected that comparisons are not always consistent and, furthermore, consistency is not a necessary condition in order for a comparison matrix to be used [48].

\subsubsection{Quality Models in Multiple Criteria Decision Making}

To this date, quality modes are used with the AHP method for the formal specification of quality criteria according to which the alternatives are compared in a recommendation process $[22,68,79,97]$. To this extent, quality models help in structuring the AHP model and in defining criteria that are important in a recommendation process in a specific field.

AHP is a method that has been widely used in software engineering [67], a field in which the need for quality models has been identified. Quality models can be defined as a set of quality characteristics, sub-characteristics, and quality measures of a product and the relationships between them [6]. They provide a common framework for quality specification and evaluation by specifying a consistent terminology for quality and by providing guidance for its measurement [8]; by following the guidance in a quality model, an evaluation is performed and evaluation results are produced according to it.

The International Organization for Standardization (ISO) identified the need for a unique and complete software quality standard and, therefore, produced the ISO 9126 standard for software quality, which was later replaced by the SQuaRE one. The SQuaRE quality model is a hierarchical quality model that describes a set of quality characteristics and their sub-characteristics related to the quality of a software product and its quality in use. The standard offers a complete view of software quality with definitions for all the quality characteristics and sub-characteristics that the standard describes.

Apart from this, various generic software quality models have been described in 
Chapter 1. Introduction

the literature: McCall's quality model [20]; Boehm's quality model [11]; the ISO 9126 quality model [61]; and the ISO SQuaRE [65] quality model. Furthermore, for various types of applications different authors have extended general quality models, such as those of the ISO, and proposed software quality models in the fields of B2B [8], mail servers [19], web-based applications [144], e-learning systems [99], ERP systems [13], web services [1], or software evolution [12].

\subsubsection{Need for Exploiting Evaluation Results and User Qual- ity Requirements}

In Multiple Criteria Decision Making, in order to provide a recommendation on the alternatives to use in a specific problem the users face, these alternatives have to be assessed and compared with respect to a set of criteria. This is usually performed by experts in the field or by users themselves. In some cases, however, it is difficult for ordinary users to make comparisons because of their lack of experience and expertise. Furthermore, although expert opinion is highly valuable in many cases, it can be expensive, and not every expert may have expertise in every alternative. Additionally, those cases when new alternatives are added require additional expert comparisons.

In some fields such as software engineering, evaluation results are available for a set of alternatives which have been evaluated either manually or automatically according to different criteria. The position adopted in this thesis is that instead of relying on expert opinions, evaluation results can be used for the automatic comparison of the alternatives, which would result in reducing the need for expensive expert opinions. Additionally, quality models, apart from being important for the specification of criteria, are also important for the evaluation of alternatives in the context of guiding in obtaining evaluation results that can be used for the comparison of such alternatives.

As mentioned in the previous section, some MCDM methods such as the AHP and the ANP require a formalized model of criteria and alternatives. In some fields, this model might contain a large number of different criteria (e.g., simulation software [54]). Arguably, not all the criteria related to alternatives are relevant for a specific user [54] and, hence, different criteria can be important for different problems that users face. This implies that for every set of criteria that are important to a specific user, a new model has to be constructed by experts, while constructing a model that is based on specific user quality requirements in terms of the most important criteria that each user is interested in can be a resource consuming task. Furthermore, any change in the model requires an effort from the experts to perform additional comparisons. 


\subsection{Semantic Technology Recommendation}

Recommendation is an important aspect in many fields in which people need to make decisions of selecting the alternative that best suites their needs. The scope of this thesis lies within one particular topic, which is that of recommendation in the semantic technology field.

\subsubsection{Semantic Technologies}

With the idea of creating a simple information and knowledge sharing system, Tim Berners-Lee proposed the creation of the World Wide Web (WWW). During the first years of the Web, sometimes referred to as Web 1.0, users were just able to consume the existing content (read-only) which consisted of documents and links that connect them. However, since its birth, the Web has significantly evolved giving its users a whole spectrum of new possibilities and usage aspects.

The term Web 2.0 has started to be widely used after the first Web 2.0 conference [96]. Although in computer science the numbers generally mark new versions of software, Web 2.0 does not mark a new version of the Web and is not related to its technical specification or new technology. It rather marks a new way in which the users use and consume the Web. It is a new generation of services that enable collaboration and content sharing, where every user has a possibility to create his or her own content (read/write Web). Some types of new services that emerged include social networks, social bookmarking, wikis, and others. But as the Web was evolving and there were more and more data present, various problems arose, like information overload and the fact that it was hard to find the right information and that information was not easy interoperable between various sources.

The Web 1.0 and Web 2.0 were designed primarily for humans. They are also called the Web of documents because they consist of a huge number of connected documents in form of HTML pages. A user that browses a Web page knows if it is about music or sports, but machines are not able to process and understand the data and their meaning. That is why search is often difficult and it is based upon keywords. In the Web (1.0 and 2.0), if a search engine is searched for a particular keyword (e.g., Madagascar) with an intention of getting information about a specific topic, there is an overload in the search results with pages about different topics that are related to that same keyword (e.g., destination and cartoon).

The Semantic Web [9] is a new generation of the Web which deals with the meaning of data in such a way that data can be understood by software agents. It is about moving from the Web of documents to a Web of data and, while the original Web was designed for humans, the Semantic Web is designed for machines (software agents). That is why in the Semantic Web a search engine is able to distinguish the 
Chapter 1. Introduction

search for a specific topic related to a particular keyword and to show relevant search results.

Semantic technologies promise to enable the development of the Semantic Web by providing new ways to express knowledge and data in machine-processable formats that can be exploited by software agents. There are different types of semantic technologies ${ }^{2}[40,95]$ which are basic components of the organization and development of Semantic Web applications [40].

One of the important characteristics of semantic technologies is that different tools belonging to various technology types can be used in different stages of the Semantic Web application development process (e.g., for ontological engineering [46] or ontology matching [32]). Furthermore, some tools also share a number of functionalities related to different technology types, as is the case of ontology engineering environments. An example of such tool is Protégé ${ }^{3}$, an ontology editor that provides additional functionalities such as reasoning (HermiT plug-in ${ }^{4}$ ) or querying (SWRL-IQ plug-in ${ }^{5}$ ).

\subsubsection{The Need for Semantic Technology Recommendation}

In the semantic technology field, different types of semantic technologies exist. For each type of semantic technology, there are various technologies (tools) that users can use for developing Semantic Web applications and, in some cases such as ontology matching tools, the number of tools can be significant with more than fifteen different tools available [33]. Because of this, the selection of semantic technologies can sometimes be a difficult task.

As previously mentioned, when developing Semantic Web applications it is often the case that tools that belong to more than one semantic technology type have to be used. This makes the selection of semantic technologies even more difficult, and in many cases users have to rely on recommendations by experts which do not have expertise in every semantic technology type.

When it comes to the AHP and the ANP methods, a number of research articles found in the literature suggest that these methods have been successfully used in the software engineering field for the recommendation of software [67]. The position adopted in this thesis is that MCDM methods, especially the ANP, can be used for the recommendation of semantic technologies. Additionally, semantic technology is a field in which plenty of evaluation results giving insight into the quality of a large number of tools are already available [41]; therefore, the exploitation of these results could help in order to provide better semantic technology recommendations. However,

\footnotetext{
${ }^{2}$ In this thesis, the term "technology" is used as a synonym for tool.

${ }^{3}$ http://protege.stanford.edu/

${ }^{4}$ http://protegewiki.stanford.edu/wiki/HermiT

${ }^{5}$ http://protegewiki.stanford.edu/wiki/SWRL-IQ
} 
one obstacle in this scenario is that, in order to provide every particular user with recommendations, experts in the MCDM field are needed for constructing an ANP model related to user-specific quality requirements.

\subsubsection{The Need for a Semantic Technology Quality Model}

Since quality models have been previously used with the AHP method [22, 68, 79, 97], the position adopted in this thesis is that a quality model for semantic technologies can be used with the ANP method for the recommendation of semantic technologies. Such quality model could help in specifying the criteria that are important in the semantic technology field and in constructing the ANP model, as well as in guiding users in the specification of the quality requirements important for their use case. However, no quality model for semantic technologies has been defined to this date.

Apart from being useful for the recommendation of semantic technologies, a quality model for semantic technologies can be a valuable asset for semantic technology quality specification and evaluation. For example, in order to benchmark different tools, which is an important aspect of evaluation [127], evaluation results from various sources need to be integrated and compared. However, since no consistent terminology for describing semantic technology quality exists, the comparison of evaluation results sometimes requires additional effort. For example, the results of a specific reasoning task obtained by Baader et al. [7] and by Glimm et al. [44] can be compared, but the fact that such results are presented with different terminology, "labeling time" and "classification time" respectively, requires a deeper analysis of the evaluation process. Furthermore, in some cases different characteristics are evaluated, and the analysis of these results or their reuse can be misleading or impossible. An example of this occurs in the analysis of reasoning times by Meditskos and Bassiliades [87] and by Urbani et al. [133]; in both cases the results are impossible to integrate because when they refer to reasoning times, they refer to different reasoning processes.

\subsection{Main Contributions}

The state of the art in MCDM, as well as the need for semantic technology recommendation, has driven the definition of the following objectives and contributions developed in this thesis:

1. To provide a Multiple Criteria Decision Making framework that extends the Analytic Network Process and that takes advantage of evaluation results of alternatives and of user quality requirements over such alternatives. To achieve this goal, the following contributions are developed in this thesis: 
Chapter 1. Introduction

- Domain-independent algorithms for the automatic comparison of alternatives according to their evaluation results. These algorithms, which can be used both in the AHP an in the ANP, provide their outputs in Saaty's pairwise comparison scale and take into account user quality requirements in terms of the most important criteria and quality thresholds.

- A set of methods for the dynamic construction of the AHP and ANP models that are based on user quality requirements. These methods and the comparison algorithms allow taking into account user quality requirements which are specified in terms of those criteria that are important for a particular user and of the desired "good to have" quality to be satisfied for the specified criteria (threshold), and together they form a base for a new MCDM recommendation framework built on top of the AHP and the ANP.

- Software that supports the proposed comparison algorithms and methods for model extraction, as well as the new MCDM framework. This software includes both a generic programming interface and a web application for performing recommendations.

2. To apply this MCDM framework in the Semantic Web field for semantic technology recommendation using existing semantic technology evaluation results. To achieve this goal, the following contributions are developed in this thesis:

- A quality model for semantic technologies defined starting from existing semantic technology evaluation results provided by the SEALS project. This quality model extends the well-known ISO SQuaRE quality model [65], describes the quality characteristics and measures that are relevant for different types of semantic technologies, and provides instructions on how to obtain those measures.

- The application of the proposed MCDM framework in the semantic technology field, including a web application for the recommendation of semantic technologies that takes into account users quality requirements and existing semantic technology evaluation results provided by the SEALS project.

\subsection{Structure of the Document}

This document is organized as follows: 
- Chapter 2 analyses the state of the art in the fields related to this thesis. First, several widely used recommendation techniques are presented and, second, a state of the art in Multiple Criteria Decision Making is described. Afterwards, some well-known software quality models are presented, followed by the review of semantic technology evaluation efforts.

- Chapter 3 enumerates the open research problems identified in the state of the art, presents the objectives and contributions of this thesis, and defines the assumptions, hypotheses and the restrictions of this thesis. Furthermore, this chapter also describes the research methodology followed in the development of this thesis.

- Chapter 4 presents the recommendation framework developed in this thesis, which is specially design to automatically exploit evaluation results and to address user quality requirements through comparison algorithms and model extraction methods.

- Chapter 5 presents the quality model for semantic technologies, and how it was developed.

- Chapter 6 presents the instantiation of the developed recommendation framework in the semantic technology field, together with a web application for semantic technology recommendation.

- Chapter 7 presents the evaluation of the main contributions of this thesis.

- Chapter 8 draws some conclusions and presents ideas for future work.

- Appendix A lists the publications used in the analysis of the state of the art of semantic technology specification.

- Appendix B describes in detail the quality model for semantic technologies developed in this thesis.

\subsection{Derived Publications}

Part of the work presented in this Ph.D. thesis was published in various peer-reviewed scientific publications belonging to conferences and journals. This section lists such publications, together with other related outcomes.

\section{- Journal publications}


Chapter 1. Introduction

- F. Radulovic, R. García-Castro and A. Gómez-Pérez. SemQuaRE - An extension of the SQuaRE quality model for the evaluation of semantic technologies. Computer Standards \& Interfaces. Volume 38. February, 2015. pp. 101-112. This publication presents the work described in Chapter 5.

\section{- Conference publications and talks}

- F. Radulovic and R. García-Castro. An ANP Based Recommendation Framework with an Application in the Semantic Technology Domain. In Proceedings of the $26^{\text {th }}$ European Conference on Operational Research (EURO2013). Rome, Italy. July, 2013. This talk presented the work described in Chapter 4 and Chapter 6 and is published in the abstract proceedings; no full publication is associated.

- F. Radulovic and R. García-Castro. Semantic Technology Recommendation Based on The Analytic Network Process. In Proceedings of the $24^{\text {th }}$ International Conference on Software Engineering and Knowledge Engineering (SEKE2012). Redwood City, California, USA. July, 2012. pp. 611-616. This publication presents the work described in Chapter 6.

- F. Radulovic and R. García-Castro. Extending Software Quality Models - A Sample In The Domain of Semantic Technologies. In Proceedings of the $23^{\text {rd }}$ International Conference on Software Engineering and Knowledge Engineering (SEKE2011). Miami, USA. July, 2011. pp. 25-30. This publication presents the work described in Chapter 5.

\section{- Workshop publications}

- F. Radulovic and R. García-Castro. Towards A Quality Model For Semantic Technologies. Software Quality Workshop 2011, within the International Conference on Computational Sciences and Its Applications (ICCSA2011). Santander, Spain. June, 2011. pp. 244-256. This publication presents the work described in Chapter 5.

\section{- Ph.D. Symposium}

- F. Radulovic. A Recommendation Framework Based on the Analytic Network Process and its Application in the Semantic Technology Domain. In Proceedings of the $15^{\text {th }}$ Conference of the Spanish Association for Artificial Intelligence (CAEPIA2013), Doctoral Consortium. Madrid, Spain. September, 2013

\section{- Recognitions}


- $3^{\text {rd }}$ Best Paper Award at the $24^{\text {th }}$ International Conference on Software Engineering and Knowledge Engineering (SEKE2012) for the Semantic Technology Recommendation Based on The Analytic Network Process publication, on July 2012.

- Software registries

- RIDER software has been registered by the Universidad Politénica de Madrid at the registry of Comunidad de Madrid, Spain (registration number M-002699/2016).

Part of the work described in Chapter 5 was also presented in the following Master thesis:

- F. Radulovic, A Software Quality Model for the Evaluation of Semantic Technologies. Master's thesis, Facultad de Informática (Universidad Politécnica de Madrid), 2011.

\subsection{Research Stays}

During the development of this Ph.D. thesis, two research stays took place at the following institutions:

- Sépage in Paris, France, from the $1^{\text {st }}$ of November to the $31^{\text {st }}$ of December of 2015. During this research stay, the author worked on applying the recommendation framework developed in this thesis for the recommendation of travel offers, under the supervision of Dr. Milan Stankovic, Sépage's CEO and Director of research. This research visit was funded by the FPU grant (EST14/00085) of the Spanish Ministry of Education, Culture and Sport (Ministerio de Educación, Cultura y Deporte).

- University of Belgrade in Belgrade, Serbia, from the $15^{\text {th }}$ of March to the $15^{\text {th }}$ of June of 2013. During this time, the author worked in the AI Lab directed by Prof. Dr. Vladan Devedzic on the development of the software implementation of the recommendation framework proposed in this thesis.

\subsection{Work in Research and Innovation Projects}

During the development of this thesis, the author has participated in the following research and innovation projects: 
- SEALS project (FP7- 238975) [1 $1^{\text {st }}$ of June of 2009 to $30^{\text {th }}$ of June of 2012]: during the author's collaboration as a Master student and as a Ph.D. student in this European project he has acquired knowledge about quality modeling, recommendation methods, and semantic technologies.

- EspOnt project (CCG10-UPM/TIC-5794) [1 $1^{\text {st }}$ of January of 2011 to $31^{\text {st }}$ of December of 2011]: during the author's collaboration as a Master student and as a Ph.D. student in this Spanish national project he has acquired knowledge about methodologies for building ontologies and experience with ontology engineering. 


\section{Chapter 2}

\section{State of the Art}

This chapter presents the work that is related to this thesis, with the purpose of providing an overview of the covered fields and of identifying open research problems. Section 2.1 describes the state of the art in the field of Multiple Criteria Decision Making methods, together with a literature review of the applications of MCDM methods in the software engineering field. Then, some well-known software quality models and existing methods for extending them are presented in Section 2.2. Afterwards, Section 2.3 describes the state of the art in semantic technology quality specification and evaluation, and Section 2.4 presents the state of the art in semantic technology recommendation. Finally, Section 2.5 presents the conclusions of the state of the art analysis.

\subsection{Multiple Criteria Decision Making}

When facing a situation in which a decision has to be made, decision makers often rely on their feelings or on experiences they had in similar situations [89]. As defined by Guitouni [49], decisions are characterized by a three angle facet:

- Rational decision is the one that consists of the evaluation of the alternatives and choosing the one that maximizes decision maker's needs.

- Non-rational decision is the one that is based on decision maker's experience and knowledge.

- Irrational decision is the one that considers only decision maker's personal aspirations and aversions.

In the cases when multiple criteria are important for making a decision, the problem becomes rather complex for a decision maker, especially if the criteria are 
Chapter 2. State of the Art

in conflict. In these cases, when facing the complex decision of selecting the best solution between a group of alternatives that can be compared according to different conflicting criteria, decision makers use Multiple Criteria Decision Making methods that help them to better structure the problem and make better choices, mainly in terms of choosing, ranking or sorting the alternatives.

Several approaches of MCDM methods are defined in the literature [34, 49, 134]:

- The outranking approach, also known as the synthesizing preference relational system approach, is an approach in which each alternative is successively compared to every other [34]. Methods based on this approach are methods in which the key concept is a degree of dominance, i.e., every alternative is observed in the context of how much it outperforms other alternatives with respect to a certain criterion. The preference of two alternatives in these methods with respect to a criterion can be defined by several relationships: preference, when one alternative is preferred over the other; indifference, when both alternatives are equally preferred; and incomparability, when two alternatives cannot be compared. These methods are also often referred to as the methods of European (French) inspiration, and include various methods such as ELECTRE, PROMETHEE, QUALIFLEX, REGIME, EVAMIX, and MELCHIOR, among others [85].

- The synthesizing criterion approach is an approach that, unlike the outranking approach, takes into account each alternative separately and assigns to each alternative a numerical value representing the overall performance (synthesizing criterion) of that alternative with respect to a set of criteria. These methods are also often referred to as the methods of American inspiration, and include various methods such as MAUT, UTA, AHP/ANP, MACBETH, TOPSIS and SMART, among others [34].

- Interactive methods are methods in which a decision maker actively participates in a decision process through expressing his or her preferences about different outcomes along various iterations in which the decision maker gains knowledge about the decision problem.

A large number of MCDM methods have been defined to date, and various MCDM methods have been successfully used in decision aiding for recommendation problems in many different fields, such as finance [124], energy planning [25], economics [143], software engineering [67], and others. However, no method is considered to be the best to be applied in every decision making problem [49, 126].

Next, this section describes the general recommendation framework followed by MCDM approaches and, afterward, it presents the most relevant MCDM methods in the literature. 


\subsubsection{General MCDM Recommendation Framework}

This section presents the general recommendation framework followed by MCDM methods (Figure 2.1). The inputs for such recommendation framework are alternatives, criteria, comparisons of alternatives according to criteria, and comparison of criteria; the output is a ranking of alternatives, i.e., an ordered list of the alternatives that are the best in overall performance with respect to the input criteria.

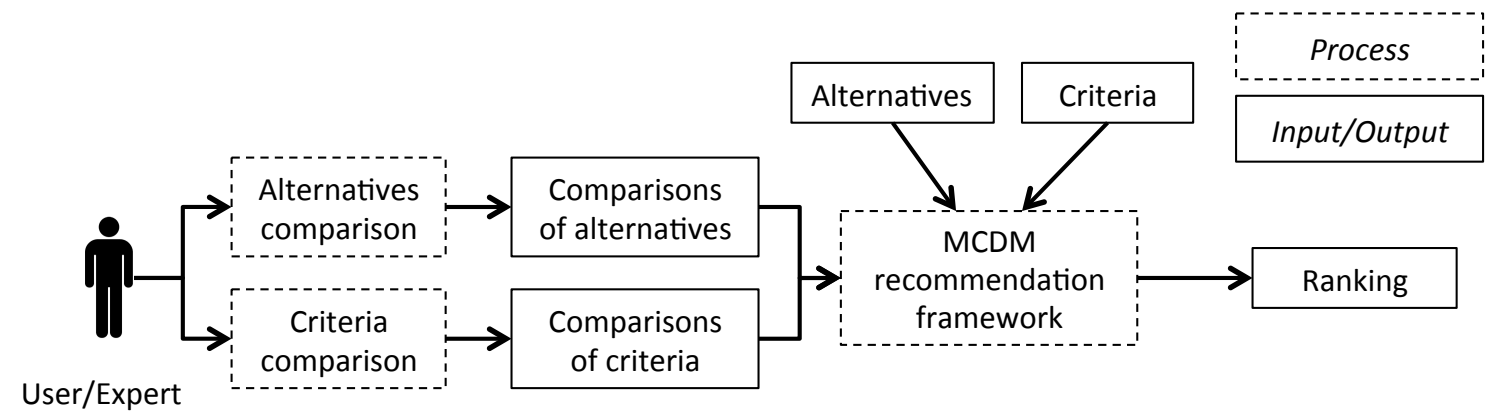

Figure 2.1: General MCDM recommendation framework.

Alternatives as inputs consist of a set of products, services, or actions, each of them suitable for solving some specific problem or for performing some specific task, and each of them belonging to the same product/service/action type. For example, a set of alternatives for the task of browsing the Web would include web browsers, such as Chrome, Firefox, Opera, etc. Criteria as inputs are quality characteristics of the alternatives that are important for the task (e.g., browser speed, memory utilization, etc.), and which are common for all the alternatives.

The comparison of alternatives and criteria is done using a comparison method which, depending on the particular MCDM method, can include either mathematical functions or pairwise comparisons, and which is often based on expert opinions or done by users. Comparison of criteria is done to determine the importance (weight) of each criterion, and comparison of alternatives according to criteria is performed to determine which alternative is the most preferred with respect to each criterion.

Finally, the output is the ranking of the alternatives that are the best according to the defined set of criteria.

\subsubsection{ELECTRE}

The ELECTRE method (ELimination Et Choix Traduisant la REalité) [108] was first developed in the sixties by Bernard Roy, in order to provide a method for helping in choosing the best action from a group of actions, and is known as ELECTRE I. It was later followed by ELECTRE II [110] and ELECTRE III [109], which were designed 
Chapter 2. State of the Art

for the problems of ranking alternatives from best to worse, and by ELECTRE IV [111], which was designed for ranking in problems in which the importance of criteria is not defined.

ELECTRE methods are outranking methods that are suitable for problems in which at least one criterion on which the actions are evaluated belongs to a scale that is not suitable for the comparison of differences (e.g., ordinal scale) [35]. Furthermore, in these methods small differences in evaluations are not significant in terms of the preferences that a decision maker has.

Decision maker's preferences are modeled with binary outranking relations with the meaning "at least as good as". Several possible situations are possible:

- When alternative $a$ is at least as good as alternative $b$ and alternative $b$ is not at least as good as $a$, then $a$ is strictly preferred to $b$.

- When alternative $a$ is at least as good as alternative $b$ and alternative $b$ is also at least as good as $a$, then $a$ and $b$ are indifferent.

- When alternative $a$ is not at least as good as alternative $b$ and alternative $b$ is not at least as good as $a$, then $a$ and $b$ are incomparable.

The outranking relations are constructed based on two key concepts: concordance and non-discordance. Concordance states that, in order for the outranking relation " $a$ is at least as good as $b$ " to hold true, a sufficient majority of criteria have to be in favor of this assertion. Non-discordance states that in the cases when the concordance condition holds, none of the criteria should be strongly opposed to the " $a$ is at least as good as b" assertion. In order to validate the " $a$ is at least as good as $b$ " assertion, both concordance and non-discordance conditions have to hold true.

The ELECTRE methods consist of two procedures: construction of outranking relations is intended to compare each pair of alternatives, while exploitation is intended for elaborating the obtained results. Furthermore, the majority of ELECTRE methods require the importance of criteria to be specified in terms of an importance coefficient, or veto threshold. The veto threshold gives a criterion the possibility to break the " $a$ is at least as good as b" assertion in some predefined situations.

\subsubsection{PROMETHEE}

PROMETHEE is another family of methods based on the outranking approach. In PROMETHEE methods [16], a preference function and a weight are defined for each criterion in the decision process.

A weight of a criterion is a non-negative number which describes its importance. Weights are defined in such a way that, for a set of $n$ criteria $\left(C_{1}, C_{2}, \ldots, C_{n}\right)$ and their corresponding weights $\left(w_{1}, w_{2}, \ldots, w_{n}\right)$, the following condition holds: 


$$
\sum_{i=1}^{n} w_{i}=1
$$

It is also possible for a decision maker to introduce arbitrary numbers as weights, which are then normalized in order to fulfill the necessary condition stated in 2.1.

For each criterion $C_{i}$, a preference function $P_{i}(a, b)$ is defined in terms of a function of a difference in evaluations $\left(F_{i}\left[d_{i}(a, b)\right]\right)$ such that

$$
\begin{gathered}
0 \leq P_{i}(a, b)=F_{i}\left[d_{i}(a, b)\right] \leq 1 \\
d_{i}(a, b)=g_{i}(a)-g_{i}(b)
\end{gathered}
$$

where $g_{i}(a)$ and $g_{i}(b)$ are evaluations of alternatives $a$ and $b$ from the set of alternatives $A$, with respect to criterion $C_{i}$. The preference function defines a degree of preference of one alternative over the other (in this case $a$ over $b$ ) for an observed difference in their evaluations on a specific criterion $\left(g_{i}\right)$. Six possible types of preference functions are defined in the literature (Figure 2.2).

Based on the preference functions, an aggregated preference indice is defined (2.4), which denotes how much one alternative is preferred over the other with respect to all criteria. The aggregated preference indice is in the range $[0,1]$ and a higher number denotes a stronger preference.

$$
\pi(a, b)=\sum_{i=1}^{n} P_{i}(a, b) w_{i}
$$

When aggregated preference indices are computed for each pair of alternatives, the positive outranking flow (2.5) and the negative outranking flow (2.6) of an alternative (e.g., alternative $a$ ) are computed. The positive outranking flow defines the degree to which one alternative outranks all the others, while the negative outranking flow defines the degree to which one alternative is outranked by all the others.

$$
\begin{aligned}
\Phi^{+}(a) & =\frac{1}{n-1} \sum_{x \in A} \pi(a, x) \\
\Phi^{-}(a) & =\frac{1}{n-1} \sum_{x \in A} \pi(x, a)
\end{aligned}
$$

The PROMETHEE I method uses the positive and negative outranking flows for the partial ranking of alternatives. By comparing the outranking flows, the preference, indifference or incomparability between two alternatives is deduced. Equality of both outranking flows implies indifference. If both positive and negative outranking flows 


\begin{tabular}{lll} 
Generalised criterion & Definition & Parameters to fix \\
\hline
\end{tabular}

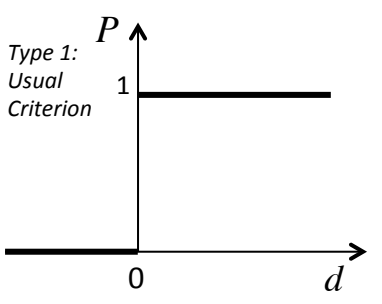

$$
P(d)= \begin{cases}0 & d \leq 0 \\ 1 & d>0\end{cases}
$$

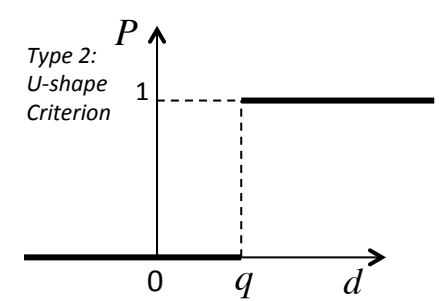

$$
P(d)= \begin{cases}0 & d \leq q \\ 1 & d>q\end{cases}
$$

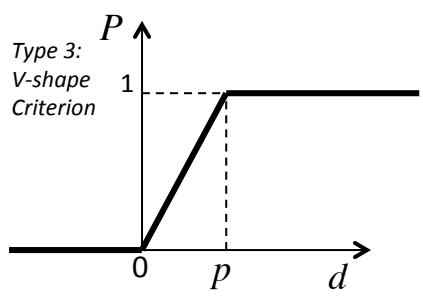

$$
P(d)=\left\{\begin{array}{ll}
0 & d<0 \\
\frac{d}{p} & 0 \leq \mathrm{d} \leq \mathrm{p} \\
1 & d>p
\end{array} \quad p\right.
$$

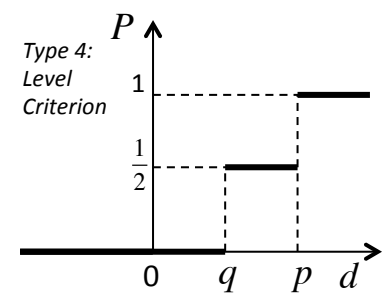

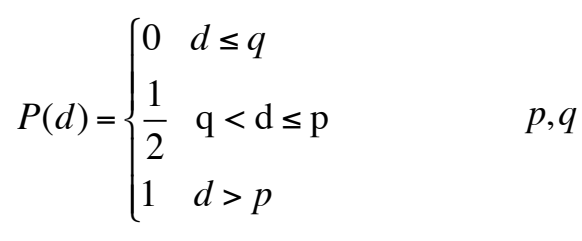

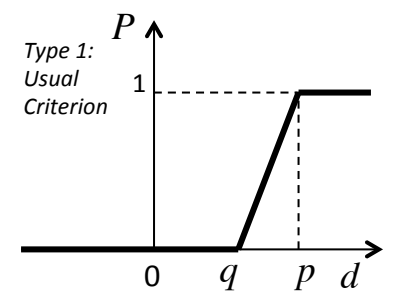

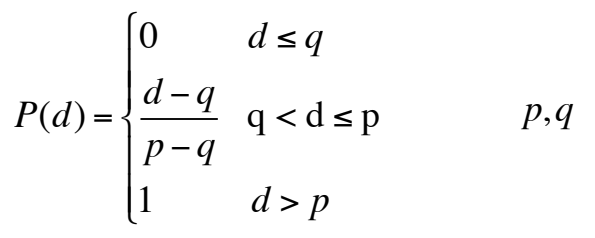

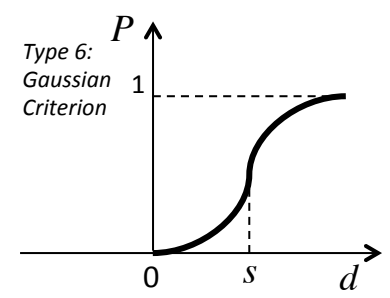

$$
P(d)=\left\{\begin{array}{ll}
0 & d \leq 0 \\
1-e^{-\frac{d^{2}}{2 s^{2}}} & d>0
\end{array} \quad s\right.
$$

Figure 2.2: Six types of preference functions in PROMETHEE methods [15]. $p$ and $q$ are thresholds of indifference and strict preference, respectively. 
of one alternative are greater or less than the outranking flows of the other alternative, then the two alternatives are incomparable. All other situations imply the preference of one alternative over the other, depending on the relation of their ranking flows.

In the PROMETHEE II method, the net outranking flow is calculated as the subtraction of the positive and negative outranking flows. Unlike in PROMETHEE I, in PROMETHEE II all alternatives are comparable, and a higher net outranking flow denotes a better alternative.

The PROMETHEE methods can only be applied when a decision maker can specify his/her preferences about a criterion on a ratio scale [70]. One of the drawbacks of the PROMETHEE methods is that they do not include any particular procedure for the calculation of the importance (weights) of criteria [84], which is an important information needed for obtaining the outranking flows.

\subsubsection{Analytic Hierarchy Process}

The Analytic Hierarchy Process (AHP) is a multiattribute utility theory method developed by Thomas Saaty [113]. The AHP is a mathematically-based method that provides the possibility of measuring both tangible and intangible criteria, and in which decisions are made based on a single score for the best decision.

The key concepts in the AHP are a pairwise comparison matrix and a fundamental comparison scale (Table 2.1); these two concepts together provide a mechanism for the comparison of alternatives in which alternatives are compared with respect to a certain criterion in order to determine which alternative is the best with respect to that criterion. To this extent, the comparison of two alternatives represents a judgment of a person on which alternative is more preferred/important for him or her. Furthermore, as the AHP requires the weights of the criteria to be specified and with the same condition as stated in equation 2.1, a pairwise comparison technique is also used for weight calculations.

An example of a pairwise comparison is shown in Table 2.2, in which three alternatives (e.g., bus, train, and plane) are compared (judged) with respect to one criterion (e.g., transportation speed). The pairwise comparison matrix is then filled with the values from the Saaty's fundamental scale in such a way that each element in the matrix denotes how much the alternative in the corresponding row of the observed

element is more important (preferred) than the alternative in the corresponding column. For example, plane is judged as extremely more important than bus and, therefore, a value of 9 is entered in the position that corresponds to plane in the row and bus in the column. According to the pairwise comparison rule, a reciprocal value is assigned in the opposite comparison. In this case, this means that when comparing bus to plane with respect to their speed, a value of $1 / 9$ is assigned and it is entered in the corresponding position in the matrix. 
Table 2.1: The AHP fundamental scale [118].

\begin{tabular}{|c|c|}
\hline $\begin{array}{c}\text { Intensity of } \\
\text { Importance }\end{array}$ & Definition \\
\hline 1 & Equal importance \\
\hline 2 & Weak \\
\hline 3 & Moderate importance \\
\hline 4 & Strong importance \\
\hline 5 & Strong plus \\
\hline 6 & Very strong importance \\
\hline 7 & Extreme importance \\
\hline 8 & $\begin{array}{c}\text { If alternative } a \text { has one of the above numbers } \\
\text { assigned to it when compared to alternative } b, \text { then } \\
b \text { has the reciprocal value when compared to } a\end{array}$ \\
\hline 9 &
\end{tabular}

Table 2.2: Example of the pairwise comparison in the AHP.

\begin{tabular}{|c|c|c|c|c|}
\hline Transportation speed & Bus & Train & Plane & Importance/Weights \\
\hline Bus & 1 & $1 / 4$ & $1 / 9$ & 0.06 \\
\hline Train & 4 & 1 & $1 / 6$ & 0.19 \\
\hline Plane & 9 & 6 & 1 & 0.75 \\
\hline
\end{tabular}

From the pairwise comparison matrix, the importance of the alternatives/criteria can be determined using various methods, one of which is the calculation of the average $^{1}$ values in each row after the matrix is normalized; this method is widely regarded as an approximation [59] of the principal right eigenvalue method developed by Saaty [112]. In the previous example, the importance of plane with respect to transportation speed is 0.75 .

Pairwise comparisons provide the means to compare alternatives by taking into account both qualitative and quantitative information. For example, in the previous comparison, the speed of transportation is qualitatively compared. On the other hand, quantitative comparison between a plane and a bus, for example, would imply that a plane is 9 times faster than the bus. Regardless of the approach taken, Saaty argues that the highest value in the comparison should not exceed the value of 9 [115], which sometimes can be an obstacle when using the quantitative approach.

\footnotetext{
${ }^{1}$ Even if some authors suggest the usage of the arithmetic mean (e.g., in [59]), due to the nature of the Saaty's scale many authors advise the usage of the geometric mean as a more precise approximation.
} 
One of the characteristics of the AHP is that it requires a decision problem to be formalized in a hierarchy of goal, criteria, and alternatives (Figure 2.3). Once such hierarchy is specified, using the pairwise comparison technique developed by Saaty, the importance of criteria is determined in one comparison and all the alternatives are compared with respect to every criterion in the model. Finally, the overall value for each alternative is calculated using formula 2.7, where $I\left(a, c_{i}\right)$ is value of the observed alternative $a$ with respect to the criterion $c_{i}$, and $w_{i}$ is the weight of the same criterion.

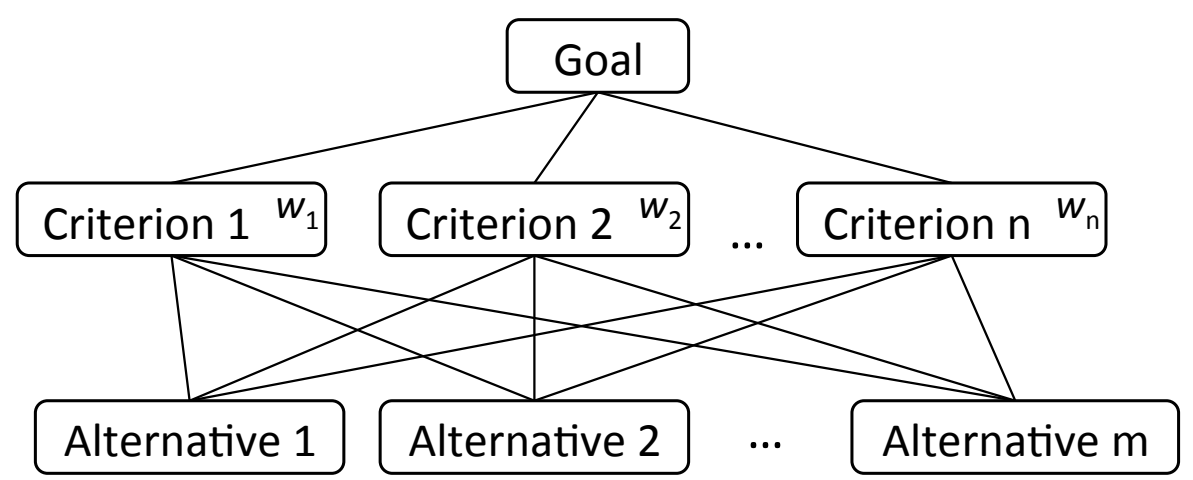

Figure 2.3: The hierarchical model of the AHP.

$$
a=\sum_{i=1}^{n} I\left(a, c_{i}\right) w_{i}
$$

In some cases, criteria in an AHP hierarchy can be present on multiple levels. In such cases, importance of criteria is determined by performing pairwise comparisons for each level of criteria separately and, then, by multiplying the importance of each leaf (lowest level) criterion with the importance of related parent criterion in order to determine the final importance of the leaf criteria. It is important to note that pairwise comparisons of leaf criteria are performed for each parent criteria separately (i.e., with respect to each parent criterion, a pairwise comparison is performed for it's child criteria).

Since pairwise comparisons represent preferences or judgments on importance over compared elements, and since inconsistencies in judgment are a natural characteristic, it can be expected that a pairwise comparison matrix could be inconsistent. In order to measure the inconsistency of pairwise comparison matrices, Saaty provides a method for consistency calculation, which is regarded as one of the main advantages of the AHP [55]. In order to calculate the consistency of a pairwise comparison matrix, a maximum eigenvalue of a comparison matrix and a consistency index (CI) are calculated first. 
The eigenvalue of a comparison matrix $A$ is a scalar $\lambda$ such that $\lambda$ is a solution to the following equation:

$$
A v=\lambda v
$$

where $v$ is a vector called eigenvector. A solution of the previous equation with respect to $v$ is an eigenvector of matrix $A$, and the scalar $\lambda$ is the related eigenvalue. One matrix can have multiple eigenvalues.

The consistency index $(C I)$ of a pairwise comparison matrix $A$ is calculated as follows

$$
C I=\frac{\lambda_{\max }-n}{n-1}
$$

where $\lambda_{\max }$ is the maximum eigenvalue of the matrix $A$ and $n$ is the matrix size (i.e., the number of alternatives/criteria that are compared in the pairwise comparison). The consistency of a pairwise comparison is then expressed through a consistency ratio $(C R)$ with the following formula:

$$
C R=\frac{C I}{R I}
$$

where $R I$ is the random consistency index, i.e., index of the randomly generated pairwise comparison matrix. Various researchers have provided values for such index [4] through simulation experiments in which pairwise comparison matrices of different sizes are randomly filled with the values from the AHP fundamental scale (Figure 2.1). In these simulations, for each matrix size separately, an average consistency index is calculated to obtain the random index. Table 2.3 shows the random indexes depending on the matrix size as calculated by Saaty.

Table 2.3: Random index [118].

\begin{tabular}{|c|c|c|c|c|c|c|c|c|c|c|}
\hline Matrix size & 1 & 2 & 3 & 4 & 5 & 6 & 7 & 8 & 9 & 10 \\
\hline RI & 0 & 0 & 0.52 & 0.89 & 1.11 & 1.25 & 1.35 & 1.4 & 1.45 & 1.49 \\
\hline
\end{tabular}

In those situations where the consistency ratio $(C R)$ is lower than 0.1 , the pairwise comparison is considered to be consistent [118], i.e., the inconsistency is considered tolerable. To deal with the situations in which comparison matrices are inconsistent, various researchers have proposed methods for changing judgments and improving consistency, such as the ones by Harker [51] (see [118]) or González-Pachón and Romero [47]. However, consistency is not considered a necessary condition in order for a comparison matrix to be used [48]. 


\subsubsection{Analytic Network Process}

The Analytic Network Process (ANP) [114] is a method which is a generalization of the AHP. It is specifically designed to take into account dependencies between criteria in the decision process and, unlike the AHP, the ANP requires the model of a problem to be formalized in a network of criteria and alternatives.

In an AHP hierarchy, criteria are mutually independent among themselves. In an ANP network, on the other hand, it is possible to account for mutual dependence between the criteria when one criterion influences some other criteria in terms of output. For example, when hiring a new employee, it could be considered that the employee's salary could depend on his or her experience, since a higher experience could imply a higher salary. Furthermore, in a network, if all candidate employees have similar experience, some criteria other than experience could gain more importance in a decision process. To this extent, the network model provides more possibilities to account for connections between the elements and is more expressive than the hierarchy model.

The ANP method consists of several consecutive steps [34, 117] (Figure 2.4):

1. The first step is to define a model of a problem, which is often referred as the most important step [69]. In the ANP, the model consists of a network of elements (criteria and alternatives) and of the dependencies between them. These elements are organized into several clusters, and dependencies between clusters are also defined in terms of arcs that connect dependent clusters. Cluster dependencies are deduced based on the existing dependencies between elements in the cluster. Different fields of application, and even different problems within

a single field, require different models which are often built by experts in the field.

Figure 2.5 shows an example of an ANP network, with elements grouped into clusters and the dependencies between them. For example, element $C_{11}$ depends on the element $C_{13}$. For the sake of simplicity, the dotted arrow denotes that each element from a cluster depends on every element from another cluster (for example, each element in the $C L_{1}$ cluster depends on both alternatives $A_{i}$ ).

2. Then, influence priorities are calculated by experts or by users through pairwise comparisons, depending on the complexity and required knowledge, and using the same principles as in the AHP.

For every particular element in the ANP network, a pairwise comparison is performed for every cluster in a network separately; each of these comparisons includes only the elements that influence the observed particular element. For example, for the network in Figure 2.5, $C_{11}$ and $C_{13}$ are compared with respect to $C_{12}$. Furthermore, pairwise comparisons are also calculated for clusters in 


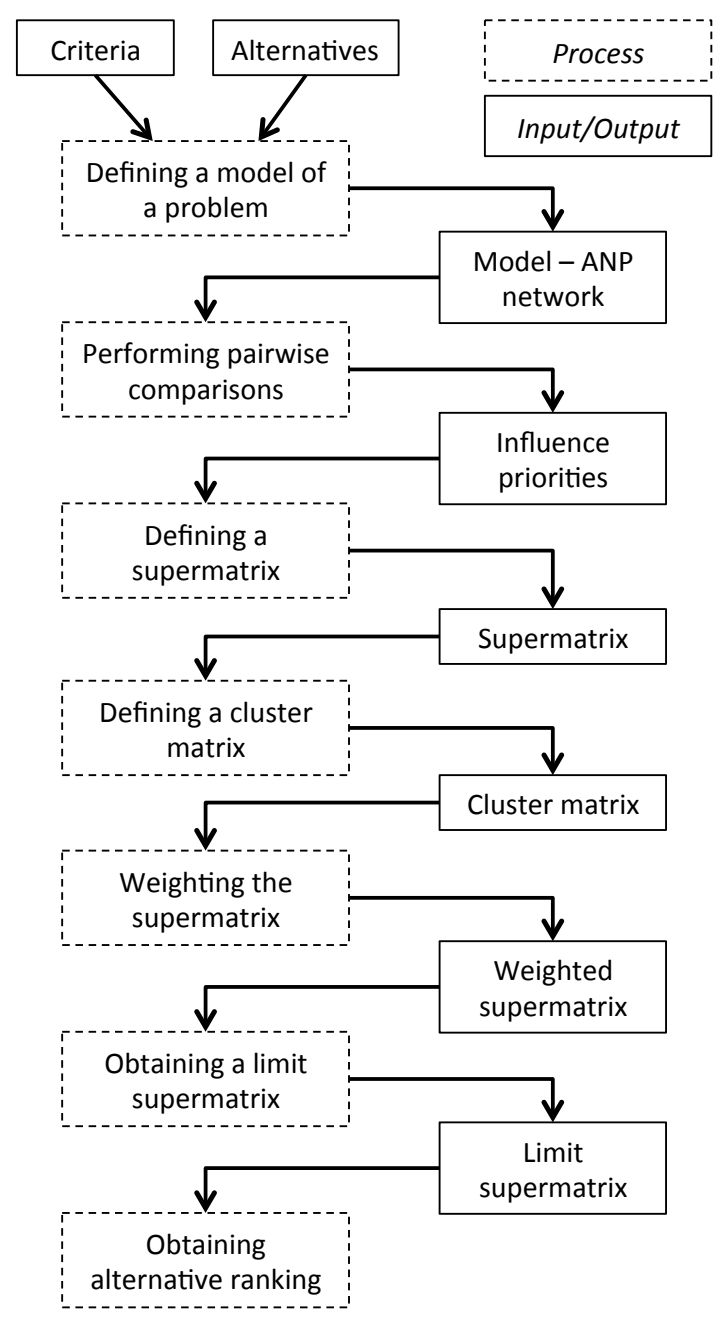

Figure 2.4: ANP workflow.

such a way that, for each cluster, a pairwise comparison is performed containing the clusters that influence it.

In the ANP, criteria can also be compared with respect to each alternative. In this case, every criteria that contributes to a certain alternative is compared to determine the level of contribution to that alternative. This step is particularly significant when observing the influence of criteria on a single alternative.

3. For the defined network, a supermatrix is formulated (see Table 2.4 for an example). The rows and columns of the supermatrix are related to the elements in the network and are grouped into the corresponding clusters. This way, a supermatrix consists of several sub-matrices, each related to two clusters in the 


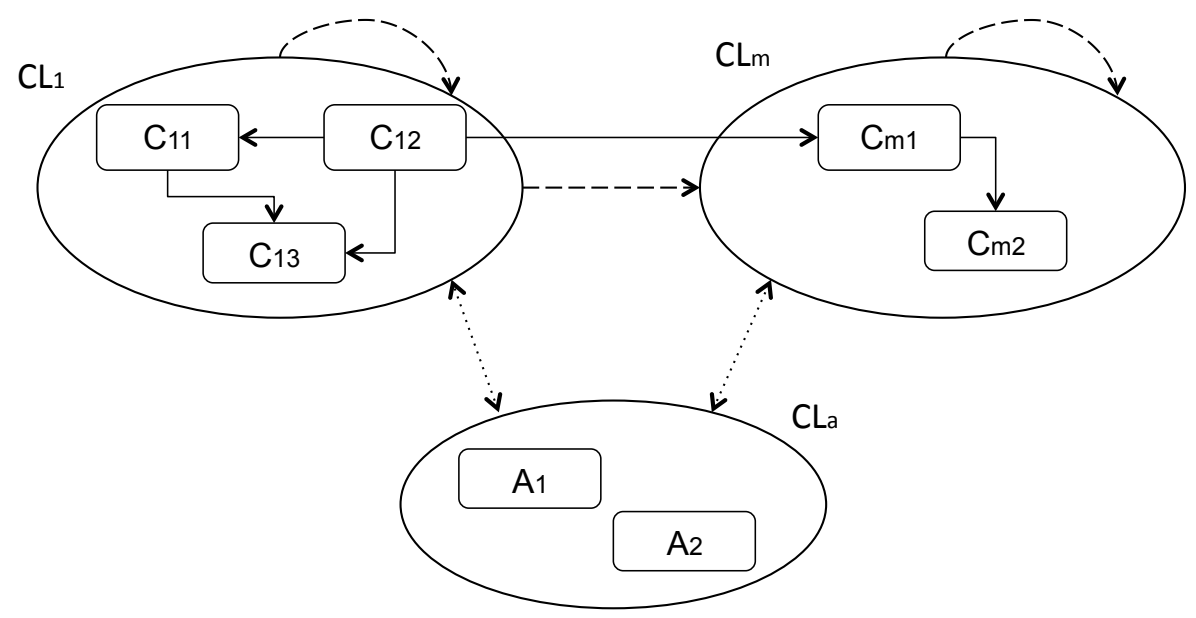

Figure 2.5: An example of an ANP network, with criteria $\left(\mathrm{C}_{i j}\right)$ and alternatives $\left(\mathrm{A}_{k}\right)$ as elements that are grouped into clusters $\left(\mathrm{CL}_{i}\right)$ and the dependencies between them.

network. The entries of the supermatrix represent the influence priorities of one element over another, e.g., the entry in the $i$-th row and the $j$-th column represents the importance of the $i$-th element for the $j$-th element. These influence priorities were calculated in the previous step for each element in every column that contains more than one element from the same cluster that influences the element in the column.

Table 2.4: Example of an ANP supermatrix, related to the network on Figure 2.5.

\begin{tabular}{|c|c|c|c|c|c|c|c|c|}
\cline { 3 - 9 } \multicolumn{2}{c|}{} & \multicolumn{3}{c|}{$C L_{1}$} & \multicolumn{2}{c|}{$C L_{m}$} & \multicolumn{2}{c|}{$C L_{a}$} \\
\cline { 3 - 9 } \multicolumn{2}{c|}{} & $C_{11}$ & $C_{12}$ & $C_{13}$ & $C_{m 1}$ & $C_{m 2}$ & $A_{1}$ & $A_{2}$ \\
\hline \multirow{3}{*}{$C L_{1}$} & $C_{11}$ & 0 & $x_{1}$ & 0 & 0 & 0 & $y_{1}$ & $q_{1}$ \\
\cline { 2 - 9 } & $C_{12}$ & 0 & 0 & 0 & 0 & 0 & $y_{2}$ & $q_{2}$ \\
\cline { 2 - 9 } & $C_{13}$ & 1 & $x_{2}$ & 0 & 0 & 0 & $y_{3}$ & $q_{3}$ \\
\hline \multirow{3}{*}{$C L_{m}$} & $C_{m 1}$ & 0 & 1 & 0 & 0 & 0 & $z_{1}$ & $w_{1}$ \\
\cline { 2 - 9 } & $C_{m 2}$ & 0 & 0 & 0 & 1 & 0 & $z_{1}$ & $w_{2}$ \\
\hline \multirow{2}{*}{$C L_{a}$} & $A_{1}$ & $f_{1}$ & $g_{1}$ & $h_{1}$ & $j_{1}$ & $k_{1}$ & 0 & 0 \\
\cline { 2 - 9 } & $A_{2}$ & $f_{2}$ & $g_{2}$ & $h_{2}$ & $j_{2}$ & $k_{2}$ & 0 & 0 \\
\hline
\end{tabular}

4. In this step the cluster matrix is constructed and contains elements related to clusters (see Table 2.5 for example). This way, each entry in the supermatrix corresponds to exactly one entry in the cluster matrix, in such a way that the clusters in the row and the column of the supermatrix that the entry belongs to determine the row and the column of the entry in the cluster matrix. The 
entries of the cluster matrix represent influence priorities calculated in pairwise comparisons in the second step.

Table 2.5: Example of an ANP cluster matrix, related to the network on Figure 2.5.

\begin{tabular}{|c|c|c|c|}
\cline { 2 - 4 } \multicolumn{1}{c|}{} & $C L_{1}$ & $C L_{m}$ & $C L_{a}$ \\
\hline$C L_{1}$ & $m_{1}$ & 0 & $b_{1}$ \\
\hline$C L_{m}$ & $m_{2}$ & $n_{1}$ & $b_{2}$ \\
\hline$C L_{a}$ & $m_{3}$ & $n_{2}$ & 0 \\
\hline
\end{tabular}

5. As the supermatrix has to be stochastic (i.e., the sum in every column has to be one), it has to be weighted. In order to weight the supermatrix, its entries are multiplied with the appropriate entries from the cluster matrix. For example, the entry related to $C_{m 1}$ in a row and $C_{12}$ in a column of the supermatrix (i.e., 1) will be multiplied with the entry related to $C L_{m}$ in a row and $C L_{1}$ in a column of the cluster matrix (i.e., $m_{2}$ ).

6. The next step is the convergence of the weighted supermatrix. The weighted supermatrix is put to a power of an increasing number, until the limit supermatrix is obtained, i.e., that in which the values in every column are equal.

7. Finally, the ranking of alternatives is obtained from the limit supermatrix. The value in every row that corresponds to an alternative represents the result for that alternative in the decision process, which is used to determine the order of alternatives. A higher value denotes a better result and is used for sorting the alternatives from best to worst.

Figure 2.6 shows a general recommendation framework that is based on the AHP/ANP methods. The ANP process showed on the figure encompasses parts of the ANP workflow (Figure 2.4).

\subsubsection{Applications of Multiple Criteria Decision Making Meth- ods in the Software Engineering Field}

This section presents a literature review of the application of MCDM methods, which has been performed following the procedure described by Kitchenham [71]. Since one of the objectives of this thesis is to provide recommendations in the semantic technology field (see Section 3.1), the focus of this literature review is on the software engineering field.

Next, the outcomes of the each step described in [71] are presented: 


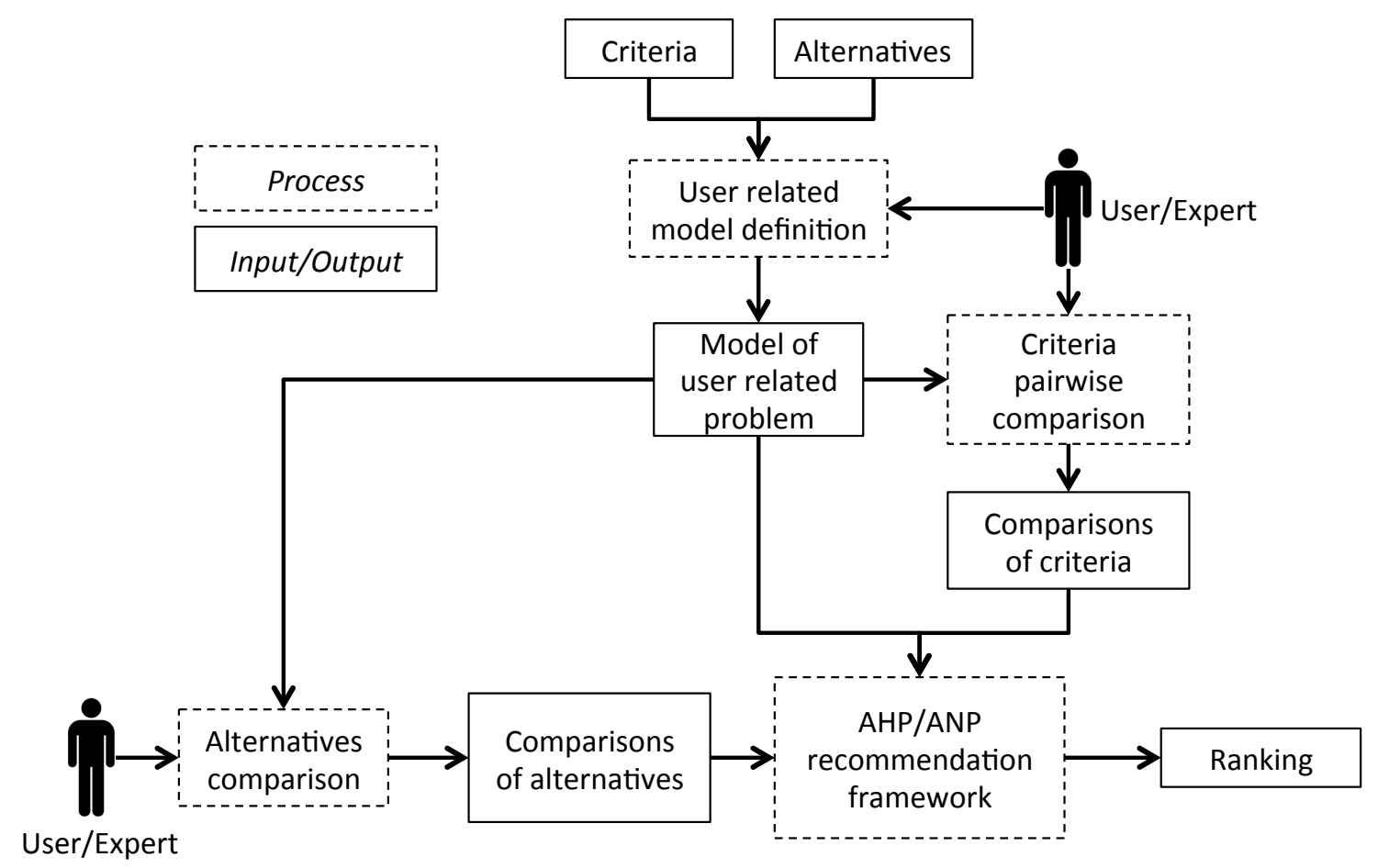

Figure 2.6: Recommendation framework based on the AHP/ANP.

- Background. The purpose of the research is to review the applications in the software engineering field of the MCDM methods described in Section 2.1, in order to obtain an overview of the current state in recommendation and selection in the software engineering field. Furthermore, the goal is to observe how each of these methods is used in practice.

- Research questions. Several research questions were stated:

- RQ1: Which MCDM methods are applied for the recommendation and selection problems in the software engineering field? This literature review will allow to observe which of the MCDM methods described Section 2.1 are most frequently used and what are their practical characteristics.

- RQ2: Which method would be the most suitable for the problem of recommendation in the semantic technology field? The goal is to observe if any of the MCDM methods can be directly used for the semantic technology recommendation problem.

- RQ3. Within the method adopted from the previous research question, is there a formal approach for the automatic exploitation of software 
evaluation results and user quality requirements? The goal is to see whether any of the existing methods, as well as solutions developed in practice, allows for the automatic exploitation of evaluation results over different scales while taking into account user quality requirements in a decision process, i.e., specific sets of criteria that are important for different users and desired "good to have" quality values over these criteria.

- RQ4: Is there an adequate software support for the exploitation of software evaluation results and user quality requirements? The goal is to observe whether there exists software support for the method to be applied in the semantic technology recommendation problem.

- Strategy for searching previous studies. In the search for research relevant to the research questions, and in order to identify those publications that deal with the application of MCDM methods in software engineering, a search was performed for publications in the leading scientific databases that offer papers from a wide number of journals, books, conferences, and workshops related to computer science. Those databases include:

- ScienceDirect ${ }^{2}$.

- IEEE Explore ${ }^{3}$.

- ACM Digital Library ${ }^{4}$.

The keywords that were used are the following: software selection, software recommendation, ELECTRE, PROMETHEE, AHP, and ANP. In the first phase of the search, all the papers were inspected with respect to their title and abstract in order to identify those publications that could help answering the stated research questions. This phase resulted in 95 publications.

- Study selection criteria and procedures. All the publications that were identified in the previous step were thoroughly inspected with the intent to select those that helped answering the research questions. The final selection was made with respect to the following criteria:

- Include only publications that deal with the MCDM methods described in Section 2.1: ELECTRE, PROMETHEE, AHP, and ANP, since those are the methods that are mostly used in the literature.

\footnotetext{
${ }^{2}$ http://www.sciencedirect.com/

${ }^{3}$ http://ieeexplore.ieee.org/Xplore/home.jsp

${ }^{4}$ http://dl.acm.org/
} 
- Include only publications that exploit one of the previously mentioned methods for the problem of recommendation, selection, or evaluation of various types of software.

- Include already conducted literature reviews that deal with the MCDM methods in software engineering.

- Data extraction strategy. While inspecting the publications selected for this literature review, the following data were extracted:

- The MCDM method that is applied.

- The type of the software that the publication deals with.

- Information of whether software support is provided.

- Information of whether real evaluation results are used for the comparison of alternatives, since evaluation results can already be found in various disciplines of software engineering, like for example in the case of semantic technologies [41, 139].

- Information of whether user quality requirements are taken into account in the recommendation.

- Any additional information that can be important for any of the MCDM methods is also extracted. This information includes whether more than one MCDM method is used, or whether a formal approach for criteria specification (i.e., a quality model) is used.

These data allowed to analyse the publications and to be able to answer the stated research questions.

- Synthesis of the extracted data. To be able to answer the research questions, the extracted data were synthesised. The synthesis was performed in the following way:

- All software types that a publication deals with are classified according to the MCDM method that is applied. This helps to observe which method is the most used one.

- Special focus is put on any specific characteristic regarding the MCDM that is applied. This helps to identify important information and possible extensions of the MCDM methods that cannot be found in books or publications with their general descriptions.

In total, forty publications were analysed, and Tables 2.6 and 2.7 show the results of this analysis. For each MCDM method that is used, the type of software 
that a publication deals with is listed together with the information about software implementations and additional notes that are important. Furthermore, existence of real evaluation results and user quality requirements are also indicated.

Table 2.6: Use of MCDM methods in software engineering (ELECTRE, PROMETHEE, and ANP).

\begin{tabular}{|c|c|c|c|c|}
\hline $\begin{array}{l}\text { Software type se- } \\
\text { lected }\end{array}$ & $\begin{array}{l}\text { Software } \\
\text { support } \\
\text { provided }\end{array}$ & $\begin{array}{l}\text { Evaluation } \\
\text { results } \\
\text { used }\end{array}$ & $\begin{array}{l}\text { User } \\
\text { require- } \\
\text { ments } \\
\text { in- } \\
\text { cluded }\end{array}$ & Notes \\
\hline \multicolumn{5}{|c|}{ ELECTRE } \\
\hline software in general [91] & no & no & yes & user-driven \\
\hline $\begin{array}{l}\text { software in general } \\
{[135]}\end{array}$ & $\begin{array}{l}\text { yes - expert } \\
\text { system }\end{array}$ & yes & yes & $\begin{array}{l}\text { use of the ISO } 9126 \text { stan- } \\
\text { dard, user-driven }\end{array}$ \\
\hline e-learning platform [86] & no & no & no & \\
\hline \multicolumn{5}{|c|}{ PROMETHEE } \\
\hline web service $[53,122]$ & no & \begin{tabular}{l|l} 
no \\
\end{tabular} & no & \\
\hline web service [103] & no & no & yes & $\begin{array}{l}\text { use of the ANP for weights } \\
\text { calculation, hard require- } \\
\text { ments }\end{array}$ \\
\hline ERP systems [105] & no & no & no & $\begin{array}{l}\text { combined with TOPSIS } \\
\text { method }\end{array}$ \\
\hline \multicolumn{5}{|c|}{ ANP } \\
\hline web services [45] & no & no & no & \\
\hline ERP systems [26] & no & no & no & \\
\hline software in general [92] & no & no & no & \\
\hline
\end{tabular}

Next, the results of the literature review with respect to each research question are discussed:

- RQ1. Which MCDM methods are applied for recommendation and selection problems in the software engineering field?

It was found that all of the methods described in Section 2.1 have been successfully applied in the software engineering field, where the AHP is the method that is used in the majority of the publications ${ }^{5}$.

- RQ2. Which method would be the most suitable for the problem of recommendation in the semantic technology field?

Although the AHP is the method that is used the most, because of the existence of dependencies between the criteria in the semantic technology field (see

\footnotetext{
${ }^{5}$ A literature review by Jadhav and Sonar [66] has similarly concluded that the AHP is the most widely applied method for software packages selection.
} 
Table 2.7: Use of MCDM methods in software engineering (AHP).

\begin{tabular}{|c|c|c|c|c|}
\hline Software type selected & $\begin{array}{l}\text { Software } \\
\text { support } \\
\text { provided }\end{array}$ & $\begin{array}{l}\text { Evaluation } \\
\text { results } \\
\text { used }\end{array}$ & $\begin{array}{l}\text { User } \\
\text { require- } \\
\text { ments } \\
\text { in- } \\
\text { cluded }\end{array}$ & Notes \\
\hline office systems [120] & no & no & yes & hard requirements \\
\hline $\begin{array}{l}\text { accounting information } \\
\text { systems [121] }\end{array}$ & no & yes & no & \\
\hline $\begin{array}{l}\text { computer operating sys- } \\
\text { tems [107] }\end{array}$ & yes & no & no & user-driven \\
\hline AHP software [97] & no & no & no & $\begin{array}{l}\text { use of the ISO } 9126 \text { stan- } \\
\text { dard }\end{array}$ \\
\hline $\begin{array}{l}\text { commercial off the shelf } \\
\text { [68] }\end{array}$ & no & no & no & $\begin{array}{l}\text { use of the ISO } 9126 \text { stan- } \\
\text { dard, AHP for calculating } \\
\text { weights }\end{array}$ \\
\hline $\begin{array}{l}\text { commercial off the shelf } \\
{[73,90]}\end{array}$ & no & no & no & \\
\hline $\begin{array}{l}\text { multimedia authorizing } \\
\text { systems }[75,76]\end{array}$ & no & no & no & \\
\hline software components [83] & yes & no & yes & user-driven \\
\hline $\begin{array}{l}\text { decision support systems } \\
{[100]}\end{array}$ & no & yes & no & \\
\hline e-commerce [119] & no & no & no & $\begin{array}{l}\text { combined with goal pro- } \\
\text { gramming }\end{array}$ \\
\hline CRM packages [22] & no & no & no & $\begin{array}{l}\text { use of the ISO } 9126 \text { stan- } \\
\text { dard }\end{array}$ \\
\hline ERP systems [136] & no & no & no & \\
\hline $\begin{array}{l}\text { knowledge management } \\
\text { tools [93] }\end{array}$ & no & no & no & \\
\hline $\begin{array}{l}\text { data warehouse systems } \\
{[82]}\end{array}$ & no & no & no & \\
\hline ERP systems [79] & no & no & no & $\begin{array}{l}\text { use of the ISO } 9126 \text { stan- } \\
\text { dard, fuzzy approach }\end{array}$ \\
\hline web services $[128,145]$ & no & no & no & \\
\hline web services [130] & yes & yes & yes & \\
\hline $\begin{array}{l}\text { online shopping platform } \\
{[57]}\end{array}$ & no & no & no & \\
\hline software architecture [24] & no & no & no & $\begin{array}{l}\text { combined with goal pro- } \\
\text { gramming }\end{array}$ \\
\hline ERP systems [80, 123] & no & no & no & fuzzy approach \\
\hline simulation software [5] & no & no & no & fuzzy approach \\
\hline $\begin{array}{l}\text { project management tools } \\
{[2]}\end{array}$ & no & no & no & \\
\hline $\begin{array}{l}\text { business process modeling } \\
\text { software [56] }\end{array}$ & no & no & no & \\
\hline simulation software [98] & no & no & no & \\
\hline management systems [30] & yes & no & yes & $\begin{array}{l}\text { fuzzy approach, user- } \\
\text { driven }\end{array}$ \\
\hline software in general [129] & no & no & no & \\
\hline
\end{tabular}


assumption 2, Section 3.3), the ANP is the method that would be the most suitable to apply in that field. Among the analysed methods, the ANP is specifically designed to tackle the problem in which criteria are dependent.

- RQ3. Within the method adopted from the previous research question, is there a formal approach for the automatic exploitation of software evaluation results and user quality requirements?

With respect to this research question, several conclusions can be outlined:

- In the large majority of publications, evaluation results are not considered and pairwise comparisons (for both ANP and AHP) are performed by experts or users. This is also the case for most cases in which some form of evaluation results is used. In their work, Seidmann and Arbel [107] used the combination of their quality assessment of alternatives and alternatives' specification in order to compare them. However, no formal approach is described and the comparisons relied on the authors' judgments. In order to make a decision on whether to use a particular decision support system or not, Phillips-Wren et al. [100] evaluate user performance for specific tasks achieved with and without the use of the decision support system in question. Based on this evaluation, a decision is made using the AHP. However, no details about the comparisons are given and about how the results are exploited in the comparisons. Finally, in some cases (e.g., [120]), authors provide use cases or illustrative examples with specified values for some criteria. However, the comparisons in these cases also rely on expert or user judgments.

In the context of the automatic exploitation of evaluation results, the work by Tran et al. [130] specified a set of general rules for comparing web services in their framework for web service ranking. Among others, the rules cover boolean results, numerical results and enumerated lists (ordinal results), and provide a mechanism for addressing user quality requirements through incorporating thresholds:

* For boolean and enumerated lists, the rules recognize which result is the same as the specified threshold and the output in the comparison is assigned based on the weight of the criterion with respect to the two alternatives being compared (e.g., when comparing two alternatives with respect to a specific criterion, if the result of one alternative is equal to the threshold, the weight of that criterion or its reciprocal value are produced as the output of the comparison, depending on which alternative is equal to the threshold). For enumerated lists, in all other cases the output is based on the ratio of the order of the 
results in the list.

* For the comparison of numerical results however, the approach relies on ratios without taking into account thresholds.

* Also, for numerical results, the specified rules distinguish the case in which the closest value to the threshold is desired. In such cases, whichever alternative that has the result that is the same as the threshold will be denoted as more preferred, assigning the reciprocal value of the weight of the criterion as the output. If two results are different than the threshold, the alternative that has the result that is closer to the threshold (regardless whether it is greater than or equal to the threshold) will be denoted as more preferred.

- User quality requirements are not always taken into account when recommending software, and the majority of applications rely on experts or users themselves to address the requirements through the definition of criteria or through judgments when performing pairwise comparisons. Addressing user quality requirements in terms of thresholds is taken into account by Seidmann and Arbel [120] through the elimination of all alternatives that do not satisfy the thresholds (hard requirements).

Tran et al. [130] provide a mechanism for addressing user quality requirements through several predefined AHP hierarchies specific to web services that can be used depending on user quality requirements. However, this approach relies on the AHP, and does not take into account dependencies between the criteria (i.e., the ANP).

The literature review showed that within the current state of the art regarding the ANP and AHP methods and their applications in the software engineering field, there is only one formal approach for exploiting real evaluation results (that of Tran et al. [130]), which produces outputs in terms of criteria weights and relies on ratios for the comparison of numerical results. Furthermore, user quality requirements are not always considered in the recommendations.

- RQ4. Is there an adequate software support for the exploitation of software evaluation results and user quality requirements?

The software that has been found in the review related to the AHP method is domain-specific and/or user-driven, i.e., it is designed to aid users of the software in exploiting the AHP method. On the other hand, the literature review showed that there is no adequate generic software support for the exploitation of software evaluation results and user quality requirements within the ANP method. 


\subsection{Software Quality Models}

It is well known that software quality is a crucial need across domains (e.g., security and health) and technologies (e.g., operating systems and databases), and that to obtain high-quality software products, the specification and evaluation of quality is of pivotal importance [8].

According to the ISO/IEC 9126 standard [61], one of the processes in the software development lifecycle is the evaluation of software products in order to satisfy software quality needs, where software quality is evaluated by measuring software attributes. Those attributes can be grouped into categories that are called quality characteristics, and these quality characteristics can be latter decomposed into quality sub-characteristics [65]. Furthermore, the ISO/IEC 9126 standard states that software product quality should be evaluated using a quality model.

The way software quality is defined depends on the approach taken [72]; software quality means different things to different people and therefore defining and measuring quality will depend on the viewpoint. Similarly, choosing one software quality model or another will also depend on the intended users and uses of such model in concrete evaluations.

Quality models provide a common framework for quality specification and evaluation by specifying a consistent terminology for quality and by providing guidance for its measurement [8]. Following the guidance in a quality model, an evaluation is performed and evaluation results are produced.

Various generic software quality models have been described in the literature. Next, this section describes some well-known software quality models and identifies their elements.

As a summary of this overview, Table 2.8 presents the elements of the described quality models.

Table 2.8: Elements of the described quality models.

\begin{tabular}{|l|l|l|l|}
\hline Structure & McCall & Boehm & ISO 9126/SQuaRE \\
\hline First level & Factor & High level characteristic & Characteristic \\
\hline Second level & Criteria & Intermediate characteristic & Sub-characteristic \\
\hline Third level & Metrics & Primitive characteristics & Measures \\
\hline Fourth level & $/$ & Metrics & $/$ \\
\hline $\begin{array}{l}\text { Relationships } \\
\text { between entities }\end{array}$ & $\begin{array}{l}\text { Factor- } \\
\text { Metric }\end{array}$ & $/$ & Measure-Measure \\
\hline
\end{tabular}




\subsubsection{McCall's Model}

McCall's software quality model is represented as a hierarchy of factors, criteria and metrics [20]. Factors are at the highest level in the hierarchy and represent the characteristics of the software product. Criteria are at the middle layer and are considered to be the attributes of the factors, so that for every factor a set of criteria is defined. At the bottom level, metrics provide measures for software attributes.

McCall's model predefines a set of eleven software quality factors that are classified according to the product life cycle into three different groups:

- Product transition: portability, reusability, and interoperability.

- Product revision: maintainability, flexibility, and testability.

- Product operations: correctness, reliability, efficiency, integrity, and usability.

McCall's quality model also gives the relationships between quality factors and metrics in the form of linear equations based on regression analyses. This is considered one of the major contributions of this model; however, the omission of the functionality aspect is regarded as the main lack [8].

\subsubsection{Boehm's Model}

Like McCall's model, Boehm's one has a hierarchical structure. In Boehm's quality model [11], there are three layers related to quality characteristics, which are also specified in the model, and one layer related to metrics:

1. High-level characteristics: as-is utility, portability, maintainability.

2. Intermediate characteristics: device-independence, self-containedness, reliability, efficiency, human engineering, testability, understandability, modifiability.

3. Primitive characteristics: accuracy, completeness, robustness, consistency, accountability, device efficiency, accessibility, communicativeness, self-descriptiveness, structuredness, conciseness, legibility, augmentability.

4. Metrics.

In total, Boehm's model describes twenty four quality characteristics and one hundred and fifty one metrics; however, the model does not provide any description of the relationships between entities. Furthermore, although Boehm's model, as McCall's one, includes aspects related to both product and users [8], McCall's model is more related to the product view while quality characteristics in the Boehm's model start from utility and are concentrated more on the users' point of view [27]. 


\subsubsection{ISO 9126's Model}

The International Organization for Standardization (ISO) identified the need for a unique and complete software quality standard and, therefore, produced the ISO 9126 standard for software quality [61]. The ISO 9126 standard gives the complete view of software quality with definitions for all the quality characteristics and subcharacteristics that the standard describes.

The ISO 9126 standard defines three types of quality:

- Internal quality: characteristics of a software product from an internal view, which are reflected in the implementation, reviewing and testing.

- External quality: characteristics of a software product from an external view, which are reflected in the execution of the software.

- Quality in use: the user's view of the software quality in terms of providing the ability for achieving specific goals.

Six main software quality characteristics for external and internal quality are specified: Functionality, Reliability, Usability, Efficiency, Maintainability, and Portability, which are further decomposed into sub-characteristics (see Figure 2.7) that are manifested externally when the software is used and are the result of internal software attributes [61]. The standard also provides the internal and external measures for sub-characteristics (see Table 2.9 for an example for sub-characteristics of Functionality).

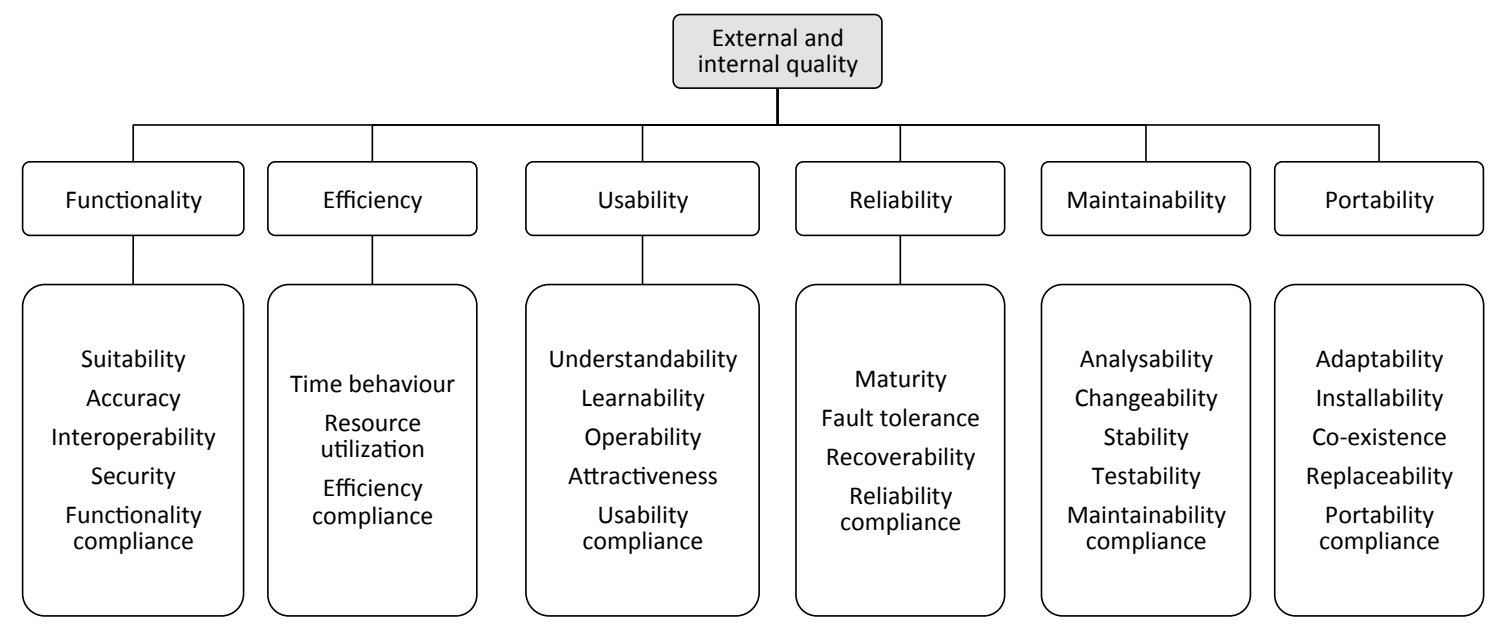

Figure 2.7: ISO 9126 internal and external quality characteristics and subcharacteristics [61]. 
Table 2.9: ISO 9126 internal and external measures for the Functionality quality characteristic $[62,63]$.

\begin{tabular}{|c|c|c|}
\hline $\begin{array}{l}\text { Quality Sub- } \\
\text { Characteristic }\end{array}$ & External Measure & Internal Measure \\
\hline \multirow{3}{*}{ Accuracy } & Computational accuracy & Computational accuracy \\
\hline & Precision & \multirow{2}{*}{ Precision } \\
\hline & $\begin{array}{l}\text { Accuracy relative to ex- } \\
\text { pectations }\end{array}$ & \\
\hline \multirow[t]{2}{*}{ Interoperability } & $\begin{array}{l}\text { Data exchangeability } \\
\text { (data format-based) }\end{array}$ & $\begin{array}{l}\text { Data exchangeability } \\
\text { (data format-based) }\end{array}$ \\
\hline & $\begin{array}{l}\text { Data exchangeabil- } \\
\text { ity (user's success, } \\
\text { attempt-based) }\end{array}$ & $\begin{array}{ll}\text { Interface } & \text { consistency } \\
(\text { protocol) } & \end{array}$ \\
\hline \multirow{4}{*}{ Security } & Access controllability & Access controllability \\
\hline & Access auditability & Access auditability \\
\hline & \multirow[t]{2}{*}{$\begin{array}{l}\text { Data corruption preven- } \\
\text { tion }\end{array}$} & $\begin{array}{l}\text { Data corruption preven- } \\
\text { tion }\end{array}$ \\
\hline & & Data encryption \\
\hline \multirow[t]{4}{*}{ Suitability } & $\begin{array}{l}\text { Functional implementa- } \\
\text { tion completeness }\end{array}$ & $\begin{array}{l}\text { Functional implementa- } \\
\text { tion completeness }\end{array}$ \\
\hline & Functional adequacy & Functional adequacy \\
\hline & $\begin{array}{l}\text { Functional implementa- } \\
\text { tion coverage }\end{array}$ & $\begin{array}{l}\text { Functional implementa- } \\
\text { tion coverage }\end{array}$ \\
\hline & $\begin{array}{l}\text { Functional specification } \\
\text { stability (volatility) }\end{array}$ & $\begin{array}{l}\text { Functional specification } \\
\text { stability (volatility) }\end{array}$ \\
\hline \multirow{2}{*}{$\begin{array}{l}\text { Functionality } \\
\text { compliance }\end{array}$} & Functional compliance & Functional compliance \\
\hline & $\begin{array}{l}\text { Interface standard com- } \\
\text { pliance }\end{array}$ & $\begin{array}{l}\text { Intersystem standard } \\
\text { compliance }\end{array}$ \\
\hline
\end{tabular}

Regarding quality in use, the model proposes four characteristics: Effectiveness, Productivity, Safety, and Satisfaction.

\subsubsection{SQuaRE's Model}

Although the ISO 9126 standard has been accepted and used successfully, some problems for its further use and improvement have been identified. They eventually arise mainly because of advances in technologies and changes of users needs. As pointed out by Azuma [6], the main problem was due to "issues on metrics" (e.g., existence of metrics that have no verified direct co-relation with quality characteristics, 
but are generally recognized as related with product quality; distribution of metrics information in several parts of the standard series, etc.).

With the object of unifying the ISO 9126 and ISO 14598 standards [60] as well as of harmonizing the content in the ISO 15939 standard [64] and taking into account the advancement in technology and environmental changes [6, 28], along with the previously described issues, the ISO 9126 standard has been redesigned and named SQuaRE (Systems and software Quality Requirements and Evaluation). The SQuaRE quality model [65] has the same hierarchical structure as the ISO 9126 one, with the difference that internal and external quality models are combined into a product quality model, which describes eight quality characteristics and thirty one quality sub-characteristics (Figure 2.8). For quality in use, SQuaRE describes five quality characteristics and nine quality in use sub-characteristics (Figure 2.9).

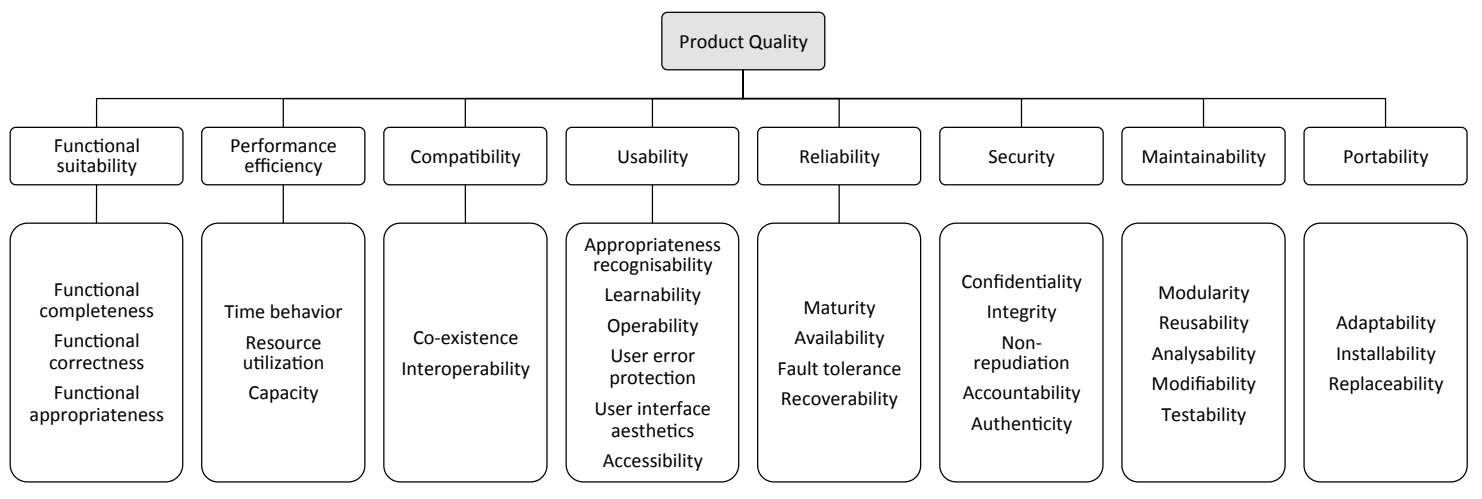

Figure 2.8: SQuaRE quality model - Product quality [65].

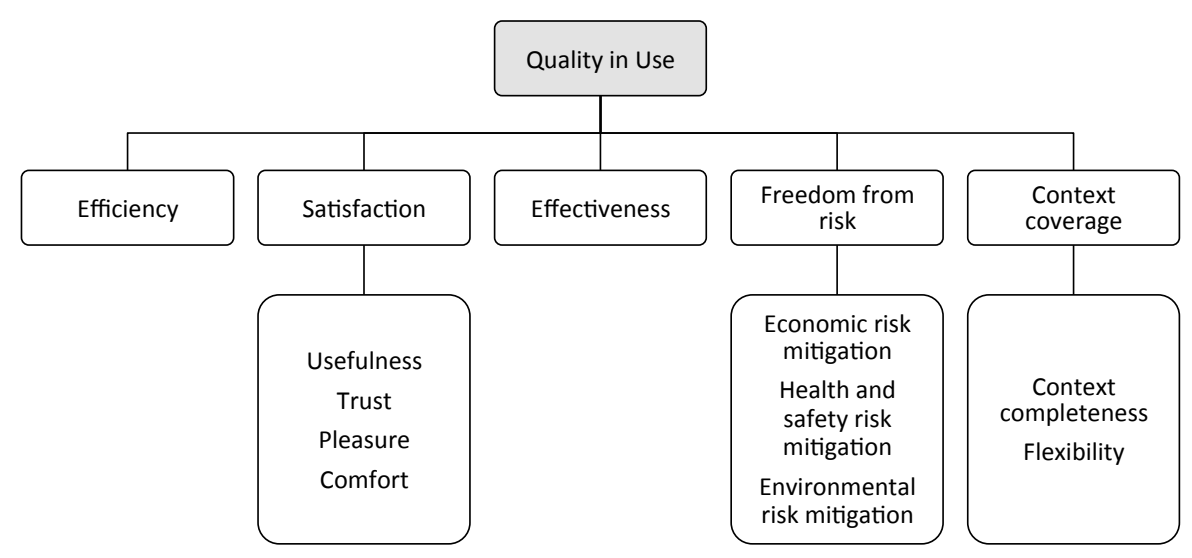

Figure 2.9: SQuaRE quality model - Quality in use [65]. 


\subsubsection{Quality Model Terminology}

According to the ISO 15939 standard [64], software evaluation is performed with the goal of capturing some information about an attribute of a software product. In this process, a measurement method is applied, and a result is a base measure (Figure 2.10), which captures some information about an attribute. Two or more base measures can be combined in a measurement function, in order to obtain derived measures. Finally, base and/or derived measures are later combined in order to obtain indicators, which are measures that provide evaluations of specific attributes. Therefore, the output of an evaluation process is a set of measures that, according to the SQuaRE standard, have to be specified in a quality model.

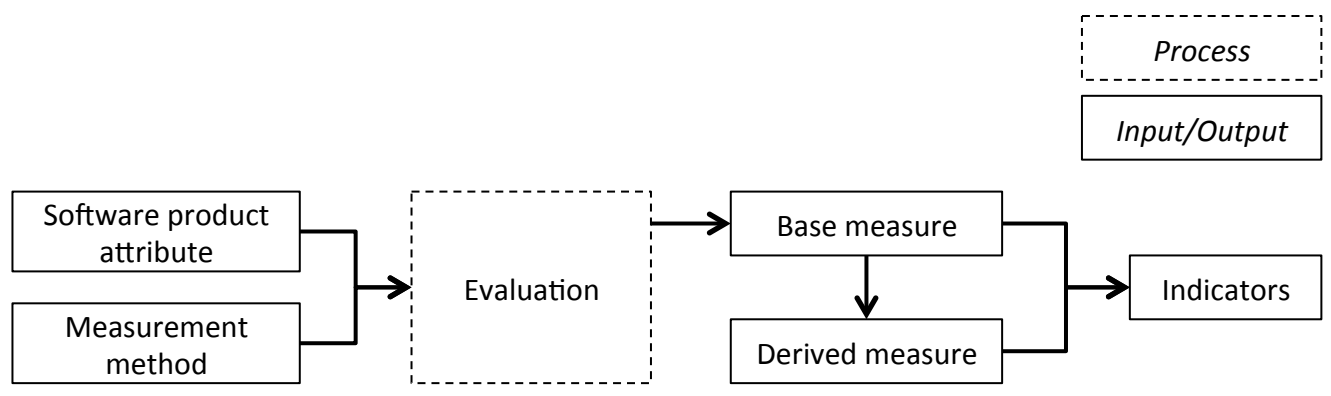

Figure 2.10: Quality model elements.

Finally, for any of those measures a measurement method and measurement scale can be defined [61]. Furthermore, a unit of measurement should be defined for all measures as well.

As different quality models use different terminology for their elements [37], the terminology adopted in this thesis is based on the SQuaRE and ISO/IEC 15939 standards. Table 2.10 presents the terminology used in this thesis.

\subsubsection{Approaches for Extending Software Quality Models}

As pointed out by Al-Kilidar et al. [3], some practical problems with ISO 9126 arise, namely, the ambiguity in metric definitions and usability interpretation. Furthermore, the authors argue that a number of attributes and measures are missing, that some characteristics are too abstract, and that the standard itself is open to interpretations which, according to the authors, questions its purpose.

Software quality models (e.g., the ISO 9126 or SQuaRE ones) provide insight into characteristics that are generic [10] and, since different types of software products have characteristics specific to them, the actual application of these software quality models usually requires refining the generic characteristics to conform with a specific software product or domain [10, 14]. To that extent, some authors suggest that, 
Table 2.10: Quality model terminology.

\begin{tabular}{|c|c|}
\hline Element & Definition \\
\hline Attribute & $\begin{array}{l}\text { Property or characteristic of an entity that can be dis- } \\
\text { tinguished quantitatively or qualitatively by human or } \\
\text { automated means [64]. }\end{array}$ \\
\hline Measurement method & $\begin{array}{l}\text { Logical sequence of operations, described generically, } \\
\text { used in quantifying an attribute with respect to a speci- } \\
\text { fied scale [64]. }\end{array}$ \\
\hline Quality measure & $\begin{array}{l}\text { Variable to which a value is assigned as the result of mea- } \\
\text { surement [64]. Quality measure includes base measures, } \\
\text { derived measures, and indicators. }\end{array}$ \\
\hline Base measure & $\begin{array}{l}\text { Quality measure defined in terms of an attribute and the } \\
\text { method for quantifying it [64]. }\end{array}$ \\
\hline Derived measure & $\begin{array}{l}\text { Quality measure that is defined as a function of two or } \\
\text { more values of base measures [64]. }\end{array}$ \\
\hline Indicator & $\begin{array}{l}\text { Quality measure that provides an estimate or evaluation } \\
\text { of specified attributes derived from a model with respect } \\
\text { to defined information needs [64]. }\end{array}$ \\
\hline Quality characteristic & $\begin{array}{l}\text { Category of software quality attributes that bears on } \\
\text { software quality [65]. }\end{array}$ \\
\hline Measurement scale & $\begin{array}{l}\text { Ordered set of values, continuous or discrete, or a set of } \\
\text { categories to which the attribute is mapped [64]. }\end{array}$ \\
\hline Unit of measurement & $\begin{array}{l}\text { Particular quantity, defined and adopted by convention, } \\
\text { with which other quantities of the same kind are com- } \\
\text { pared in order to express their magnitude relative to that } \\
\text { quantity [64]. }\end{array}$ \\
\hline
\end{tabular}

according to the nature of the product itself, some new sub-characteristics can be added, the definitions of existing ones can be changed, or some sub-characteristics can be eliminated from the model [13]. By following these suggestions, the previously mentioned problems can be overcome.

For various types of applications, different authors have proposed software quality models in the domains of B2B [8], mail servers [19], web-based applications [144], e-learning systems [99], and ERP systems [13]. All those authors have used the ISO 9126 standard as the basis software quality model and have extended it to conform with their particular domain. Additionally, some authors have extended the SQuaRE quality model in the domains of web services [1] and software evolution [12].

Software quality model extensions can be performed following two main approaches [29]: 
- A top-down approach that starts from the quality characteristics and continues towards the quality measures.

- A bottom-up approach that starts from the quality measures and defines the quality sub-characteristics that are related to each specific measure.

To this date, only two methods for extending software quality models have been found in the literature and both follow a top-down approach. These two methods are described next, using the original terminology employed by their authors.

1. Franch and Carvallo proposed a method based on a top-down approach for customising the ISO 9126 quality model [36], which they latter applied in constructing the quality model for mail servers [19]. After defining and analysing the domain of interest, their method proposes six steps:

(a) To determine quality sub-characteristics. In this first step, according to the domain, some new quality sub-characteristics are added while others are excluded, or their definitions are changed.

(b) To define a hierarchy of sub-characteristics. If needed, sub-characteristics are further decomposed according to some criteria.

(c) To decompose sub-characteristics into attributes. In this step, abstract sub-characteristics are decomposed into more concrete concepts that refer to some particular software attribute (i.e., observable feature).

(d) Decomposing derived attributes into basic ones. Attributes not directly measurable are further decomposed into basic ones.

(e) To state relationships between quality entities. Relationships between quality entities are explicitly defined. Three possible types of relationships are identified:

- Collaboration means that increasing the value of one entity implies increasing the value of another entity.

- Damage means that increasing the value of one entity implies decreasing the value of another entity.

- Dependency implies that some values of one entity require that another entity fulfills some conditions.

(f) To determine metrics for attributes. To be able to compare and evaluate quality, it is necessary to define metrics for all attributes in the model.

2. In building their quality model for B2B applications, Behkamal et al. proposed a method to customize the ISO 9126 quality model in five steps [8]. The main difference with the previous method is that in Behkamal's approach, the quality 
characteristics are ranked by experts; thus, experts should provide weights for all quality characteristics and sub-characteristics and these weights are later used to establish their importance, which can be time consuming and resource demanding. Besides, Behkamal's approach does not contemplate defining relationships between quality entities (quality characteristics and attributes).

\subsection{Semantic Technology Quality}

Quality is an important concern in every domain and with the increasing adoption of semantic technologies in recent years multiple evaluations of such technologies have been proposed in order to assess their quality, from general and qualitative evaluation frameworks [95] to tool-specific evaluations [50, 77] and even to characteristic-specific evaluations [39]. This section describes the current state regarding the semantic technology quality specification, as well as regarding the quality characteristics and quality measures evaluated in the semantic technology field.

\subsubsection{Semantic Technology Quality Specification}

Different types of semantic technologies have been described in the literature [40, 95]; the scope of this thesis is limited to five types of semantic technologies, which do not exhaustively cover every type of semantic technology. Next, a short description of the types that are relevant to this thesis is given:

- Ontology engineering tools are software for managing ontologies and their components either through a user or a programming interface.

- Ontology matching tools are software programs for finding correspondences (i.e., alignments) between the components of two ontologies.

- Reasoning systems are software programs for inferring logical consequences from ontology instances.

- Semantic search tools are software programs that provide answers to natural language queries by exploiting data semantics.

- Semantic web service tools are software programs that support the use of semantic web services (i.e., web services enriched with semantic descriptions) for different tasks (e.g., discovery, composition and mediation, among others).

Multiple evaluations of semantic technologies have been performed to this date, both conducted by individuals and by the evaluation campaigns driven by the 
community. In order to obtain the current state of specification of semantic technology quality, a literature review has been performed.

This section presents such a review on semantic technology evaluations, which was performed according to the procedure described by Kitchenham [71].

Next, the outcomes of the each step described in the procedure are presented:

- Background. The purpose of the research is to review previous evaluations of semantic technologies, including their evaluation results, in order to obtain the overview of the current state in specification of semantic technology quality for the five selected types of tools.

- Research questions. Two research questions were stated:

- RQ1: Is there a common approach for specifying the quality of semantic technologies? This literature review will allow to observe if there is a unique reference or guideline followed by researchers to specify semantic technology quality.

- RQ2: Which semantic technology characteristics are evaluated and which quality measures are used to evaluate them? The goal is to observe the quality characteristics and measures used in different evaluations, as well as the terminology that is used.

- Strategy for searching previous studies. In the search for the relevant research to the stated research questions, and in order to identify those publications that deal with semantic technology evaluation, an analysis of the proceedings of the two most relevant conferences and of the three workshops in the Semantic Web area that are focused on the topic of evaluation has been performed. Those proceedings are:

- International Semantic Web Conference (ISWC) - eleven editions (20022012).

- European/Extended Semantic Web Conference (ESWC) - nine editions (2004-2012).

- International workshop on Evaluation of Ontology-based Tools (EON) all six editions (2002-2004;2006-2008).

- International Workshop on Evaluation of Semantic Technologies (IWEST) - all two editions (2010, 2012).

- International workshop on Scalable Semantic Web Knowledge Base Systems (SSWS) - eight editions (2005-2012). 
In the first phase of the search, all papers were inspected with respect to their title and abstract, in order to identify those publications that can help answering the stated research questions. This phase resulted in 179 publications.

- Study selection criteria and procedures. All publications that were identified in the previous step were thoroughly inspected with the intent to select those that help answering the research questions. The final selection was made with respect to following criteria:

- Include only publications that deal with the following types of semantic technologies: ontology engineering tools, reasoning systems, ontology matching tools, semantic search tools, and semantic web service tools.

- Include publications that discuss or present measures for semantic technology evaluation.

- Include publications that report on evaluations of existing semantic technologies.

- Include publications that suggest new algorithms (e.g., for reasoning or semantic web service discovery), which are evaluated in the publication.

- Data extraction strategy. While inspecting the publications taken for this literature review, the following data related to research questions have been extracted:

- Type of semantic technology that is evaluated.

- Measures used in the evaluation. Measures are extracted in their original terminology, which means that the different terminologies that different authors use for the same measure is taken into account.

These data allowed to analyse the publications and to be able to answer the stated research questions.

- Synthesis of the extracted data. To be able to answer the research questions, extracted data have been synthesised. The synthesis has been performed in the following way:

- Measures that are extracted in their original terminology are classified according to measure that is evaluated. This helps to observe the use of terminology and to see whether different terms are used for the same measure.

- Each extracted measure is aligned to a quality characteristic from SQuaRE, and all the measures extracted in the review are classified according to 
those characteristics and according to the types of semantic technologies. This helps to observe which quality characteristics are evaluated.

- Frequencies of the number of times each measure and characteristic appears are also taken into account. This helps to observe which measures and characteristics are the most common.

In total, one hundred publications were analysed ${ }^{6}$; the list of publications is given in Appendix A. Tables 2.11, 2.12, 2.13, 2.14, and 2.15 show the results of the analysis for ontology engineering tools, ontology matching tools, reasoning systems, semantic search tools and semantic web service tools, respectively. Each table shows the number of papers in which a specific type of semantic technology is evaluated, together with the quality characteristics (shown in bold), which were extracted from the SQuaRE standard, that are evaluated for the related technology type. For every characteristic found in the literature for particular semantic technology type, a set of measures that can be used for measuring such characteristic is presented and, in those cases where different terminology was used, measures are referred to by the names used in the literature for them and are grouped under an arbitrary common name (shown in italics) that semantically denotes the measure. For example, classification correctness for reasoner systems is found in one publication as "number of successes" and in another publication as "number of solved tasks" and, therefore, these two measures are grouped together under "classification correctness". In some cases, however, it was not possible to determine the exact classification (e.g., in the case of time behavior of reasoning systems). The number of papers in which a specific measure was found and the number of papers that evaluated a specific characteristic are shown in brackets. Numbers are omitted when only one occurrence appears.

The analysis of the current state in semantic technology evaluation helped in answering the research questions stated in the literature review, which are shown next:

- RQ1. Is there a common approach for specifying the quality of semantic technologies?

Table 2.11: Characteristics and measures used in conference publications for ontology engineering tools.

\begin{tabular}{l}
\hline Ontology engineering tools (8) \\
\hline Functional correctness (4) - precision (2), recall (2), information added, information lost, bench- \\
marks passed \\
Interoperability (3) - information addition/loss (3) [changes performed, import/export, knowl- \\
edge preserved, knowledge lost] \\
Time behavior (2) - execution time (2) \\
Maturity (1) - execution
\end{tabular}

\footnotetext{
${ }^{6}$ The complete analysis of publications is available for download at http://goo.gl/LzWuOT
} 
Table 2.12: Characteristics and measures used in conference publications for ontology matching tools.

Ontology matching tools (35)

Functional correctness (32) - precision (31), recall (30), f-measure (22) [f-measure (19), f-score

(3)], weighted symmetric difference, found correspondences

Time behavior (4) - matching time (4) [execution time, time, mapping time, runtime]

Maturity (1) - error rate

Resource utilization (1) - memory consumption

Table 2.13: Characteristics and measures used in conference publications for reasoning systems.

Reasoning systems (31)
Time behavior (26) - classification time (11) [classification time (5), time (3), classification perfor-
mance, total time, labeling time, lattice operation time, query time], query execution time (5) [query
time (2), query response time, query answering time, time], entailment time (5) [time for entailment,
time, justification time, reasoning time, kernel running time], data loading time (2) [loading time
(2)], ontology loading time [loading time], closure time (2) [runtime, closure time], execution time,
cpu time, response time, runtime, time cost, loading time
Functional correctness (11) - classification correctness (4) [number of successes, number of cor-
rect classifications, number of wrong classifications, number of solved tasks, classifications], entail-
ment correctness (3) [number of solutions per second, soundness, logical entailment], satisfiability
correctness (2) [number of SAT instances solved, class satisfiability, ontology satisfiability], number
of matches (2), number of refinements, effectiveness, gain, answer completeness
Resource utilization (2) - memory usage, network traffic
Maturity (2) - resources exceeded, errors, failure

Table 2.14: Characteristics and measures used in conference publications for semantic search tools.

Semantic search tools (15)
Functional correctness (11) - precision (9), recall (8), NDCG (2), f-measure (3) [f-measure (2),
f-score], reciprocal rank, system accuracy, relevance, number of correct queries and answers
Time behavior (10) - query execution time (5) [time (2), query response time, running time, exe-
cution time], question time (2) [time, question time], input time (2), system speed, task completion
time, execution time, response time
Operability (5) - system usability scale (SUS) questionnaire (4), search experience
Satisfaction (3) - extended questionnaire (2), user satisfaction
Efficiency (2) - answer found rate (2), number of attempts (2), experiment time (2)
Learnability (1) - learning

Table 2.15: Characteristics and measures used in conference publications for semantic web services.

Semantic web service tools (11)

Functional correctness (8) - precision (8), recall (8), f-measure, number of target sources returned, BPREF, reciprocal rank, success at cut-off point, cumulated gain

Time behavior (5) - discovery time (5) [processing time, query answering time, query response time, execution time, discovery time] 
It has been found that no common approach for specifying the quality of semantic technologies exists and that neither quality model nor guideline has been developed in this domain. Furthermore, of all the publications analysed, it has been observed that only one publication provides instructions on the measures to be evaluated. In this publication [94], the authors propose a set of novel measures for ontology matching tools: comprehensive F-measure, STD score and ROC-AUR score, which have never been used before in evaluations. Five publications provide some discussion on existing measures that have been used before, of which three are related to reasoning systems [21, 127, 141], one to semantic web services [74], and another to ontology matching tools [131].

- RQ2. Which semantic technology characteristics are evaluated and which quality measures are used to evaluate them?

Tables 2.11 to 2.15 directly show all the information that is related to the characteristics that are evaluated and to the quality measures used. The following conclusions can be outlined:

- When observing a specific type of semantic technology, it can bee seen that there is a variety of quality characteristics that are evaluated; i.e., in different publications different characteristics are evaluated. For example, while in some publications the time behavior of semantic web services is evaluated, in others only correctness is. Furthermore, different quality characteristics are evaluated across different types of semantic technologies.

- When studying specific quality characteristics, it can be seen that there is a variety of measures for measuring them. This means that, although some publications evaluate the same quality characteristic, they measure it with different measures. An example of this can be the classification and satisfiability evaluation of reasoning systems.

- Different publications use different terminologies for measures so their names are different even when they evaluate the same aspect. This can be directly observed in the previously shown tables (e.g., in Table 2.13 for the case of classification correctness of reasoning systems).

\subsubsection{Semantic Technology Evaluation Results}

The Semantic Evaluation At Large Scale (SEALS) project was an FP7 $7^{7}$ project [139] which created the infrastructure for semantic technology evaluation and performed evaluations of semantic technologies through public evaluation campaigns.

\footnotetext{
${ }^{7}$ http://cordis.europa.eu/fp7/home_en.html
} 
Two public evaluation campaigns of the SEALS project have produced evaluation results for several types of semantic technologies. For each type of semantic technology, several tools were evaluated according to common test data in one or several evaluation scenarios:

- Three evaluation scenarios were defined for ontology engineering tools:

- Conformance scenario.

- Interoperability scenario.

- Scalability scenario.

In total, six ontology engineering tools were evaluated in the first evaluation campaign [42] and nine ontology engineering tools were evaluated in the second evaluation campaign [43].

- Five evaluation scenarios were defined for ontology reasoning systems:

- Classification scenario.

- Class satisfiability scenario.

- Ontology satisfiability scenario.

- Entailment scenario.

- Non-entailment scenario.

In total, three ontology reasoning systems were evaluated in the first evaluation campaign[140] and four ontology reasoning systems were evaluated in the second evaluation campaign [142].

- One evaluation scenario was defined for ontology matching tools:

- Accuracy scenario.

In total, fifteen matching tools were evaluated in the first evaluation campaign [132] and nineteen matching tools were evaluated in the second evaluation campaign [88].

- Two evaluation scenarios were defined for semantic search tools:

- User-in-the-loop scenario (accuracy and performance).

- Automatic scenario (accuracy, performance, usability).

In total, five semantic search tools were evaluated in the first evaluation campaign [137] and eight semantic search tools were evaluated in the second evaluation campaign [138]. 
- One evaluation scenario was defined for semantic web service tools:

- Semantic web service discovery.

In total, four semantic web service tools were evaluated in the first evaluation campaign [18] and two semantic web service tools were evaluated in the second evaluation campaign [78].

Table 2.16 shows an overview of the measures used in the first and the second SEALS evaluation campaigns.

Table 2.16: Measures used in the first and the second SEALS evaluation campaigns.

\begin{tabular}{|l|}
\hline Ontology engineering tools \\
\hline $\begin{array}{l}\text { execution problems, importing-exporting duration, information added, information } \\
\text { lost, structural equivalence, semantical equivalence }\end{array}$ \\
\hline Ontology matching tools \\
\hline precision, recall, f-measure, runtime, errors \\
\hline Reasoning and storage systems \\
\hline classification time, class satisfiability time, ontology satisfiability time, entailment \\
time, non-entailment time, loading time, classification correctness, class satis- \\
fiability correctness, ontology satisfiability correctness, entailment correctness, \\
non-entailment correctness, classification errors, class satisfiability errors, ontology \\
satisfiability errors, entailment errors, non-entailment errors \\
\hline Semantic search tools \\
\hline $\begin{array}{l}\text { loading time, load successful, query time, number of completed queries, number } \\
\text { of results, time per result, precision, recall, f-measure, experiment time, usability } \\
\text { value, satisfaction value, number of attempts, answer found rate, input time }\end{array}$ \\
\hline Semantic web service tools \\
\hline $\begin{array}{l}\text { precision, recall, binary preference, reciprocal rank, number of retrieved documents, } \\
\text { number of relevant documents, number of relevant documents retrieved, normalized } \\
\text { discounted cumulative gain }\end{array}$ \\
\hline
\end{tabular}

\subsection{Semantic Technology Recommendation}

In the semantic technology domain, there are different types of technologies that users can use in order to solve a problem. Furthermore, for some problems users often have to use more than one type of semantic technology. Therefore, the quality requirements that users might have with respect to semantic technologies cover criteria across tools which can be satisfied either by a single tool or by a combination of them. 
Selecting the best technologies among those that users can use in order to solve a specific problem can be a difficult task, especially for users with no or little expertise in the semantic technology field. In such field, to the best of the knowledge of the author of this thesis there is only one work related to providing a recommendation framework, that of Mochól [89]. However, her work deals with ontology matching tools, which is only one type of semantic technologies.

In her work, Mochól takes into account matching approaches and algorithms, employed by different tools, and provides a framework for their manual or semiautomatic selection. The manual approach relies on the Analytic Network Process, and requires manual pairwise comparisons of matching approaches (i.e., alternatives) to be performed by experts, and the manual definition of the model and pairwise comparisons of criteria by users. The semi-automatic approach, on the other hand, is based on rules that compare whether the characteristics of a certain matching approach match the characteristics of the input ontologies.

\subsection{Conclusions to the State of the Art}

A large variety of MCDM methods have been developed to date and they have been successfully applied in many different fields for recommendation problems, including software engineering.

In MCDM recommendation problems, comparisons between alternatives are usually performed by experts in the field or by users themselves. In some cases, however, it is difficult for ordinary users to make comparisons because of their lack of expertise. Furthermore, although expert opinion is highly valuable in many cases, it can be expensive and not every expert may have expertise in every alternative. Also, those cases when new alternatives are added or existing alternatives evolve require additional expert comparisons.

In those cases where the evaluation of alternatives has already been performed and evaluation results are available, it can be useful to ground the comparisons of alternatives in such evaluation results. In AHP pairwise comparisons, as suggested by Saaty, the comparison of numerical results that belong to ratio scale can be based on ratios. Such approach for numerical results is also adopted in the rules defined by Tran et al. [130]. However, a possible problem is that in the case of largely separated values, the comparisons would fall out of the Saaty's scale. Also, in the case of values that are not significantly separated, the comparisons might not provide enough differentiation between the alternatives. Furthermore, the rules defined by Tran et al. rely on criteria weights as outputs instead of on the Saaty's scale. Such approach implies that the strength of preference between two alternatives will be greater if the criterion is less important, and vice versa. To this extent, no formal approach based on Saaty's fundamental scale for the automatic exploitation of real 
evaluation results across different scales has been found. Finally, different users might have different quality requirements in a recommendation problem and user quality requirements are not always taken into account.

As discussed before, the quality of software products is of high importance; therefore, the specification and evaluation of quality is of great concern. Software quality models provide the basis for quality specification and evaluation; however, since there is neither a unique specification nor a quality model for semantic technologies, the current state of semantic technology evaluations is characterized by a large variety of characteristics, measures, and terminology of the quality evaluated. A possible consequence of this variety is that the integration and analysis of different evaluation results (e.g., when performing benchmarks or comparisons), which can be particularly significant for the selection of tools according to their quality, might require additional effort or lead to misleading and wrong conclusions. For example, the results of a specific reasoning task obtained in Baader et al. [7] and in Glimm et al. [44] can be compared, but the fact that such results are presented with different terminology ("labeling time" and "classification time", respectively) requires a deeper analysis of the evaluation process. Also, the comparison of reasoning times in Meditskos and Bassiliades [87] and in Urbani et al. [133] can be misleading because when they refer to "reasoning time", they refer to different reasoning processes. Therefore, without a common ground to specify the quality of semantic technologies, it is very difficult to compare them and to assess their quality.

Using a software quality model may provide a good common basis for evaluation, since software quality models support the specification of quality and instruct on how to measure it. Existing software quality models provide terminology and description of characteristics that are general for almost every kind of software. However, in order to use a quality model in a specific domain, it usually has to be extended to include the particularities of such domain $[10,14]$.

Various methods for extending quality models have been proposed in the literature; they all follow a top-down approach, starting from general characteristics to concrete measures. As reported by Botella et al. [13] in their work with the top-down method, identifying some measures can be a difficult task and it is not always possible to identify all of them. Furthermore, they outline that metrics are also very difficult to define for some measures and having rigorous metrics is a prerequisite for reliable comparisons between products [61].

By the time of writing this thesis, the author has not found any example of a bottom-up approach in the general literature. In some scenarios, however, it can be helpful to base the extension of the quality model on existing software evaluations and evaluation results, when these are available. Furthermore, some authors suggest that a bottom-up approach is important when building quality models [29]. 
Quality models have been successfully used to date with MCDM methods in recommendation problems. In the Software Engineering field, and in the case of the AHP, which requires a hierarchical structure in its model, hierarchical software quality models (e.g., ISO 9126 or SQuaRE) are very convenient, and different authors have used software quality models based on ISO 9126 together with the AHP [22, 68, 79, 97]. 


\section{Chapter 3}

\section{Work Objectives}

This chapter presents the main objectives of this thesis and the open research problems it aims to address (Section 3.1), together with its main contributions (Section 3.2). Furthermore, the chapter also presents the work assumptions, hypotheses, and restrictions of this thesis (Section 3.3), as well as the plan for the evaluation of the stated hypotheses (Section 3.4). Finally, Section 3.5 presents the research methodology and research process followed in this thesis.

\subsection{Thesis Objectives and Open Research Problems}

The objectives of this thesis aim to advance the current state of the art in the Multiple Criteria Decision Making field and in the Semantic Web field; on the one hand, by providing a Multiple Criteria Decision Making framework that extends the Analytic Network Process and that takes advantage of evaluation results of alternatives and of user quality requirements over such alternatives and, on the other hand, by applying this MCDM framework in the Semantic Web field for semantic technology recommendation using evaluation results.

In order to achieve the first objective, from a methodological perspective the following list of open research problems must be solved:

- In some cases, at the time of making a decision over some alternatives (i.e., products, services or actions), these alternatives have already been evaluated according to a set of criteria and evaluation results are available; those results can be useful for the comparison of alternatives in a decision process because they provide an insight into the quality of the alternatives and can be used for deriving preferences between alternatives. However, in the ANP method (and, consequently, in the AHP one) comparisons are usually made manually 
by experts or users, and only one formal approach for comparisons has been found, which is based on criteria weights. To this extent, there is a lack of a method that is based on the Saaty's fundamental scale and that enables the automatic comparison of alternatives based on alternatives' evaluation results across different scales.

- A decision process is usually performed by experts manually, or in some cases semi-manually with the help of appropriate software. In each decision making process, a set of criteria related to alternatives is specified and a decision is made having in mind these criteria. However, since different users have different quality requirements in terms of desired criteria and desired values for these criteria, different alternatives are best suited for each user (i.e., for each different set of user quality requirements). Currently, user quality requirements are not always considered and there is a lack of a generic method in the ANP method that enables automatic recommendations based on different sets of user quality requirements over alternatives.

From a technological perspective the following engineering problems must be solved:

- Current software implementations of the ANP and of the AHP are domain-specific or do not support the automatic exploitation of evaluation results and user quality requirements. Furthermore, current generic software implementations require previous knowledge on these methods. Also, the comparison of alternatives has to be made manually by the users of the software, and every user needs to construct the model (network or hierarchy) based on his or her quality requirements.

In order to achieve the second objective, from a methodological perspective the following open research problem must be solved:

- Quality models provide a specification of quality characteristics and the quality measures needed for the evaluation of such characteristics. Such models are used with decision making methods as a reference for the specification of user quality requirements. However, in the current state of the art a quality model for semantic technologies has not been defined yet.

From a technological perspective the following engineering problem must be solved:

- With a large number of semantic technologies that belong to different types of semantic technologies, and with the common need to use more than one technology to solve a certain problem, choosing the best semantic technology 
or set of technologies (e.g., tools when developing an application) is a difficult task for users. Currently, there is a lack of an application that provides recommendations over semantic technologies based on user quality requirements and on the available evaluation results of semantic technologies.

\subsection{Contributions to the State of the Art}

This thesis aims at giving solutions to the previous open research and engineering problems. Chapter 4 describes the solution proposed for the first objective (the development of a MCDM framework that exploits alternative evaluation results and user quality requirements) and Chapters 5 and 6 present the solutions related to the second one (semantic technology recommendation).

With regard to the first objective, this thesis has contributed to the advance of the current state of the art by providing RIDER: a MCDM framework based on the ANP, that takes into account evaluation results of alternatives and user quality requirements defined over such alternatives. In particular, this thesis presents new advances by providing the following:

C1. Domain-independent algorithms for the automatic comparison of alternatives according to alternatives' evaluation results, based on Saaty's scale. These algorithms, which can be used both in the AHP and in the ANP, take evaluation results and user quality requirements as inputs and provide their outputs in Saaty's pairwise comparison scale. To this extent, these algorithms represent the simulation of alternative comparisons performed by the experts or users. Furthermore, the algorithms are based on the Euclidean distance (for numerical results), which is often used for the calculation of similarity between values, and on values matching (for nominal and ordinal results).

With respect to the current state of the art, this contribution provides advancement in terms of new comparison algorithms, based on Saaty's pairwise comparison scale, that enable the automatic exploitation of evaluation results.

C2. A set of methods for the dynamic extraction of the AHP and ANP models that are based on user quality requirements. These methods and the comparison algorithms $(C 1)$ allow taking into account user quality requirements in the decision process.

In this thesis, the term user quality requirement denotes a criterion important for a particular user and a desired value (threshold) to be satisfied for this criterion. Therefore, user quality requirements and thresholds denote a "good 
to have" and not a hard condition or a must that would exclude alternatives if not satisfied.

With respect to the current state of the art, this contribution provides advancement in terms of a formalized approach for addressing user quality requirements, through the AHP and the ANP model extraction, while taking into account dependencies.

C3. Software that supports the proposed MCDM framework. This software includes both a generic programming interface (jRIDER) and a generic web application (RIDER-WebApp) for performing recommendations.

With respect to the current state of the art, this contribution provides advancement in terms of a generic software support that automatically delivers recommendations by exploiting the AHP and the ANP methods and is, therefore, able to take into account dependencies.

The second objective of this thesis is to apply the previous MCDM framework for semantic technology recommendation. To this extent, this thesis presents new advances by providing the following:

C4. SemQuaRE, a quality model for semantic technologies that has been defined starting from existing semantic technology evaluation results. This quality model extends the well-known ISO SQuaRE quality model, describes the quality characteristics and measures that are relevant for different types of semantic technologies, and provides instructions on how to obtain those measures.

With respect to the current state of the art, this contribution provides advancement in terms of a quality model for semantic technologies which, to the best of the author's knowledge, has not been previously defined.

C5. The application of the RIDER framework in the semantic technology domain, including a web application for the recommendation of semantic technologies that takes into account user quality requirements and existing semantic technology evaluation results obtained in the SEALS project (Section 2.3.2).

With respect to the current state of the art, this contribution provides advancement in terms of a system for delivering semantic technology recommendations to users which, to the best of the author's knowledge, has not been previously developed.

Contributions $C 1, C 2$, and $C_{4}$ are related to the methodological perspective, while contributions $C 3$ and $C 5$ are related to the technological perspective. 


\subsection{Work Assumptions, Hypotheses, and Restrictions}

This thesis has been developed under a set of assumptions that help explain the decisions taken for the achievement of the thesis goals; such assumptions are listed bellow. As it can be observed, assumption A1 is related to the RIDER MCDM framework presented in this thesis, while assumptions A2, A3, and A4 deal with the application of the RIDER framework in the semantic technology domain:

A1. Quality models provide a good basis for the specification of user quality requirements in a decision process.

Since quality models formally specify evaluation guidelines and the characteristics to be evaluated, they can be useful as a reference for the possible quality requirements that users take into account in a decision process.

A2. Some quality characteristics of semantic technologies are dependent on each other.

This assumption is expected because dependencies between some quality characteristics in software products are common and some researchers have already addressed dependencies in the Software Engineering field [26, 45, 92].

A3. The ANP is the most suitable among all the MCDM methods that can be applied for the recommendation of semantic technologies.

Since the ANP is particularly useful for the recommendations in which dependencies exist, and since dependencies are present in the semantic technology domain (see A2), the ANP can be considered as the most suitable among all the MCDM methods for the application in the semantic technology domain.

A4. The evaluation results for semantic technologies produced in the SEALS project are correct.

All the tools evaluated in the SEALS project were evaluated against the same execution environment and using the common input data. Therefore, it can be assumed that the evaluation results produced in SEALS are correct.

Once the assumptions have been identified and presented, the set of hypotheses of this thesis are described. Hypotheses $\mathrm{H} 1$ to $\mathrm{H} 5$ are related to the RIDER MCDM framework, while hypotheses H6 to H9 deal with the application of the RIDER framework in the semantic technology domain:

H1. The outputs of the algorithms for the comparison of alternatives that exploit evaluation results do not depend either on the distribution or the range (distance between the minimum and maximum values in the results) of such evaluation results. 
H2. The quality of the algorithms for the comparison of alternatives that exploit evaluation results does not improve when more evaluation results are available.

H3. User quality requirements (in terms of thresholds) do not influence the comparison of alternatives performed by the algorithms.

H4. User quality requirements (in terms of thresholds) do not help discriminating between alternatives.

H5. Those alternatives that satisfy a higher number of specified user quality requirements are ranked better in an extracted model which only contains those criteria appearing in specified user quality requirements.

H6. The SemQuaRE quality model for semantic technologies is representative, which means that it covers the majority of the quality characteristics that are evaluated in practice.

H7. The SemQuaRE quality model for semantic technologies is flexible, which means that it can be extended with new quality characteristics or new types of tools.

H8. The RIDER framework in the ontology matching domain produces recommendations that are accurate in the context of users' needs.

H9. The RIDER framework in the ontology matching domain produces recommendations that satisfy users.

Finally, the following set of restrictions defines the limits of the contributions of this thesis and allows determining future research objectives. These restrictions delimit the research problem and allow the incremental improvement of research. Restrictions $\mathrm{R} 1$ and R2 are related to the RIDER MCDM framework, while restrictions R3, R4 and R5 deal with the application of the RIDER framework in the semantic technology domain:

R1. The algorithms for the comparison of alternatives are based on Saaty's pairwise comparison method and a fundamental scale, which is used in the AHP and ANP methods.

R2. The methods for the dynamic extraction of the AHP and ANP models that are based on user quality requirements only cover hierarchical and network models. Other types of models are not taken into account.

R3. User quality requirements that can be used for semantic technology recommendation cover only those quality characteristics evaluated in the SEALS project, i.e., those characteristics for which the evaluation results are available. 
R4. With respect to the web application for semantic technology recommendation, the available alternatives and their evaluation results cover only those alternatives and results provided by the SEALS project.

R5. The evaluation of the RIDER framework in the semantic technology domain only covers ontology matching tools.

Figure 3.1 shows the mappings between the objectives described in Section 3.1, the contributions described in Section 3.2, and the assumptions, hypotheses and restrictions described in this section.

\subsection{Evaluation Plan}

With respect to the hypotheses defined in this thesis, in order to accept or reject them, the following evaluations have been performed (Chapter 7):

E1 (for H1). The goal of this evaluation is to determine the influence of the distribution and range of evaluation results on the comparisons of alternatives provided by the comparison algorithms. To this end, the results of comparing alternatives using different variations of the comparison algorithm, by varying the distribution and range of input results, can be compared to one another to observe how the distribution and range of results influence the outputs of the algorithm.

E2 (for H2). The goal of this evaluation is to determine the influence of the quantity of evaluation results on the quality of the comparison algorithms. To this end, the results of comparing alternatives using different variations of the comparison algorithm (i.e., using partial or full sets of evaluation results) can be compared.

E3 (for H3). The goal of this evaluation is to determine the influence of user quality requirements on the comparison of alternatives provided by the comparison algorithms. To this end, a set of comparisons obtained without specifying any quality requirements can be analysed and compared to a set of comparisons obtained with respect to a number of quality requirements, in order to determine the influence of user quality requirements (in terms of thresholds) on comparisons.

E4 (for H4). The goal of this evaluation is to determine the influence of user quality requirements on discrimination between alternatives. To this end, similarly as with the previous evaluation, a set of comparisons obtained without specifying any quality requirements can be analysed and compared to a set of comparisons 


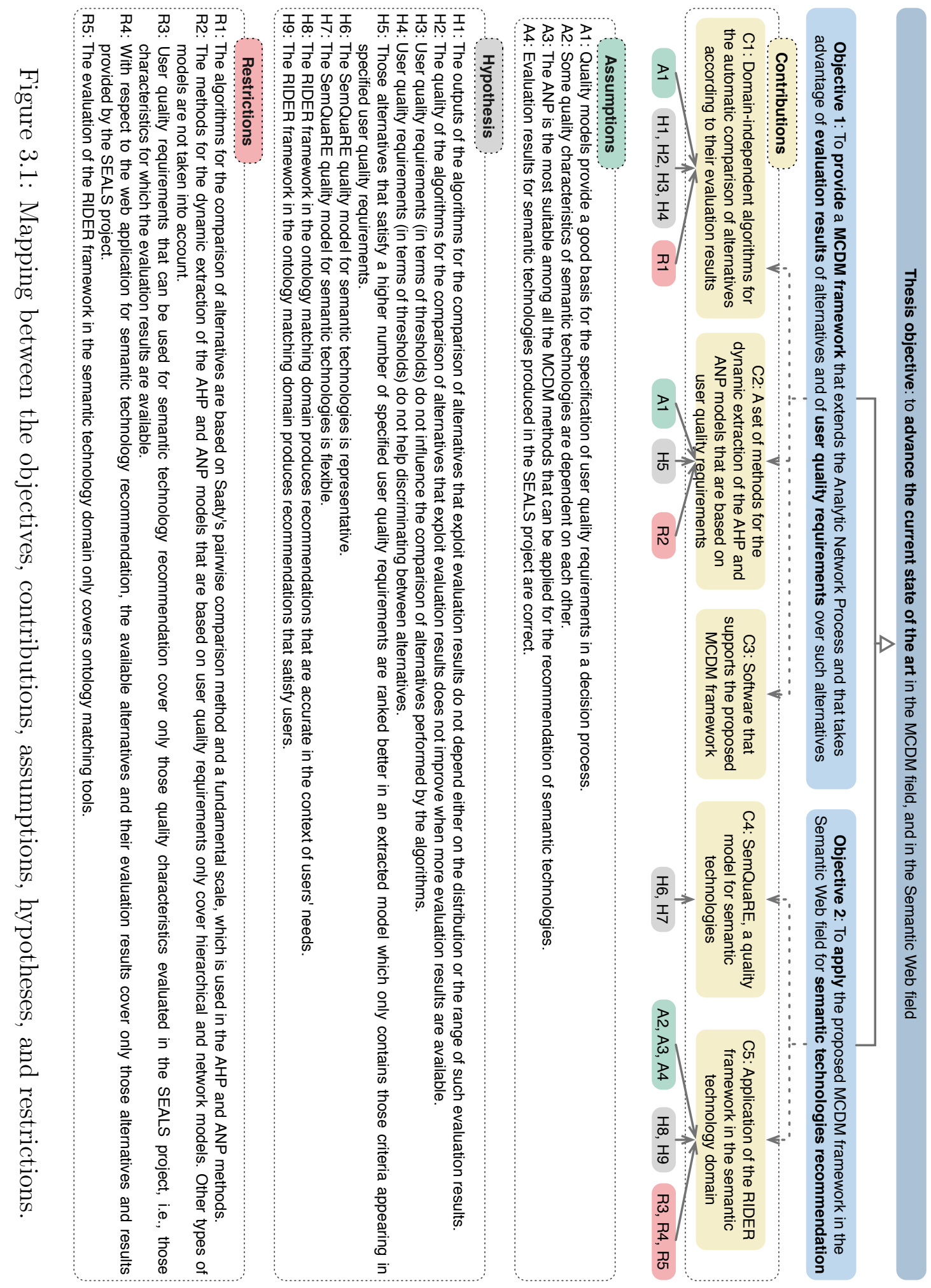


obtained with respect to a number of quality requirements, in order to determine the differences in discrimination between alternatives.

E5 (for H5). The goal of this evaluation is to determine how model extraction methods influence the recommendations provided by the RIDER framework. To this end, recommendations obtained with different extraction methods can be compared, in order to determine how the extraction methods influence the recommendations.

E6 (for H6). The goal of this evaluation is to determine the representativeness of the quality model for semantic technologies. To this end, a literature review on semantic technology evaluation can show which quality characteristics are evaluated, and the results of such review can be compared to the quality characteristics described in the quality model.

E7 (for H7). The goal of this evaluation is to determine the flexibility of the quality model for semantic technologies. To this end, the flexibility of the quality model can be examined through its extensions with both new quality characteristics and with new types of semantic technologies.

E8 (for H8). The goal of this evaluation is to determine whether the application of the RIDER framework to the semantic technology domain, in particular its application to the ontology matching domain, can produce recommendations that are accurate in the context of users' needs. To this end, the ontology matching tool recommendations obtained with the instantiation of the RIDER framework in the ontology matching domain can be compared with respect to the recommendations provided by users themselves.

The reason for limiting this evaluation to the ontology matching tools domain is because it is the domain in which the largest number of tools is evaluated (nineteen tools evaluated with respect to five criteria) and with the highest variability in evaluation results. This allowed for choosing a subset of five tools in order to design the evaluation in such a way that the number of tools and criteria does not require high complexity in order for the interviewed users to perform recommendations.

E9 (for H9). The goal of this evaluation is to determine whether the application of the RIDER framework to the semantic technology domain, in particular its application to the ontology matching domain, can produce recommendation that are satisfactory to users. To this end, the ontology matching tool recommendations obtained with the instantiation of the RIDER framework in the ontology matching domain can be presented to users, which would be asked to provide feedback on their satisfaction with the presented recommendations. 


\subsection{Research Methodology}

This section presents an overview of the research methodology followed during the development of this thesis, as well as the followed research process (Section 3.5.1).

The approach for achieving the objectives of this thesis (Section 3.1) consists of providing several contributions to open research problems, as described in Section 3.2. These contributions are then combined together in order to achieve each specified goal.

In order to deliver the contributions of this thesis, methodological and technological inputs are taken into account as presented in Figure 3.2. Furthermore, in developing this thesis' contributions, community based feedback has been taken into account. Figure 3.2 also shows that the work in this thesis is framed by a set of objectives, assumptions and restrictions presented in this chapter.

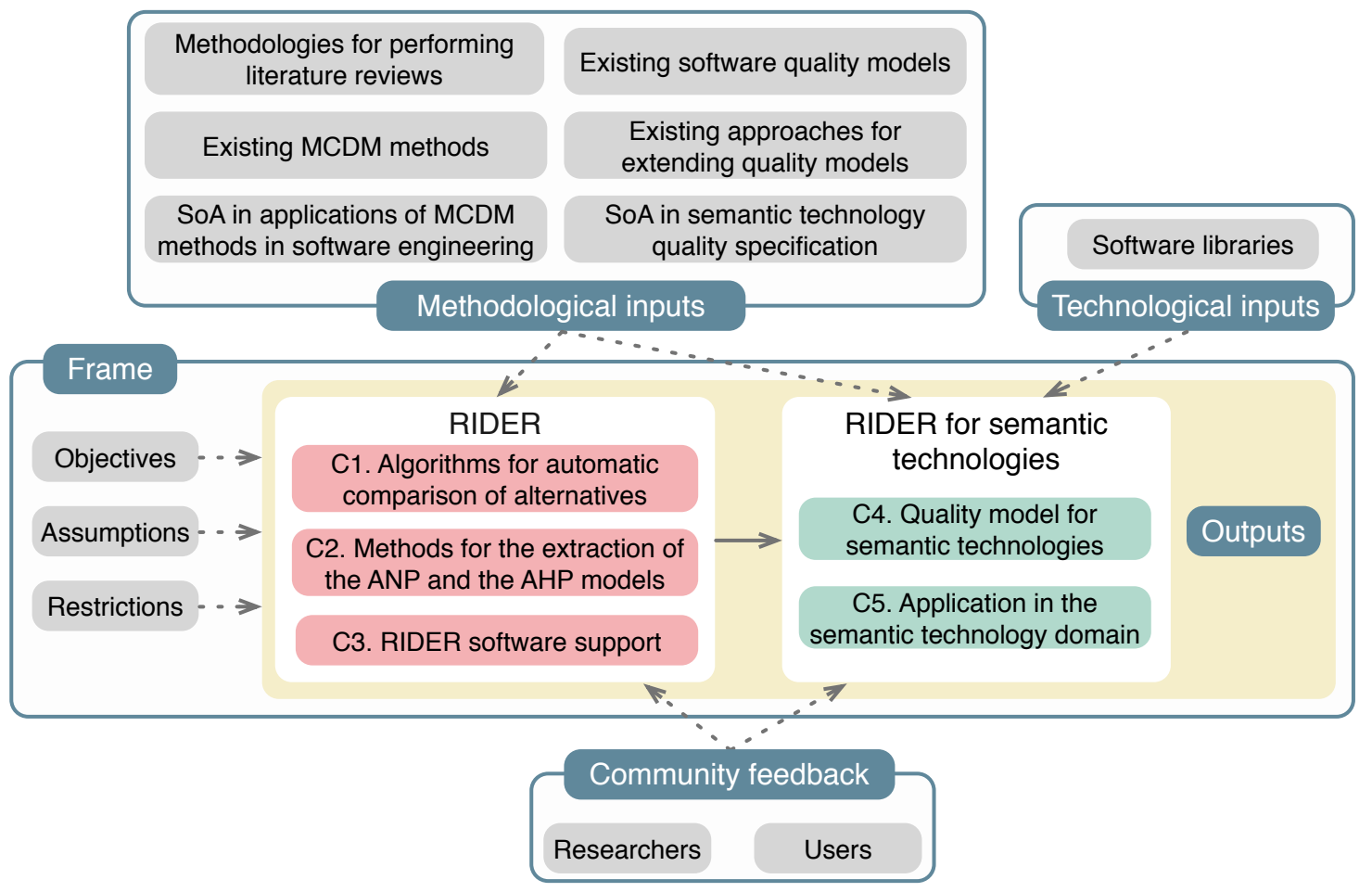

Figure 3.2: Inputs taken into account for delivering thesis' contributions.

In order to deliver contributions related to the objective of providing a Multiple Criteria Decision Making framework that extends the Analytic Network Process and that takes advantage of evaluation results of alternatives and of user quality requirements over such alternatives, which is called RIDER, the following inputs were taken into account: 
1. Methodological guidelines for performing literature reviews. Part of the methodology followed in the thesis relies on the literature reviews performed in the fields of interest. For performing such literature reviews, methodological guidelines by Kitchenham [71] have been followed.

2. Existing Multiple Criteria Decision Making methods. Existing MCDM methods, presented in Section 2.1, were analysed in order to obtain an overview of the MCDM field and methods.

3. State of the art analysis in Multiple Criteria Decision Making applications in the Software Engineering field. This analysis, presented in Section 2.1.6, together with the previous input helped in identifying open research problems related to the MCDM field, and the most suitable MCDM method for the Software Engineering field to be used as a basis for the new recommendation framework presented in this thesis.

4. Software libraries. For developing software to support the MCDM framework proposed in this thesis, existing software libraries such as Jena, Spring and Tapestry were used.

5. Community feedback. In the development of this goal, feedback from researchers was taken into account, mostly through dissemination of the results as described in Section 1.5 and through participations at various international and national conferences and workshops.

In order to provide contributions related to the objective of applying the proposed MCDM framework in the Semantic Web field for semantic technology recommendation, i.e., of providing RIDER for semantic technologies, the following inputs were taken into account:

1. Existing software quality models. Existing software quality models, presented in Section 2.2, were analysed in order to obtain an overview of generic software quality models used in Software Engineering.

2. Existing approaches for extending software quality models. Existing approaches for extending software quality models, presented in Section 2.2.6, were analysed in order to obtain an overview of the process and steps in the specification of domain-specific quality models.

3. State of the art analysis in semantic technology quality specification. This analysis, presented in Section 2.3.1, helped in identifying open research problems related to quality specification and evaluation in the semantic technology field.

4. Community feedback. In the development of this goal, feedback from users of the semantic technology recommendation framework was taken into account. 


\subsubsection{Description of the Research Process}

In the development of this thesis, an incremental process has been followed leading to four different phases: initialisation, development, implementation, and refinement. Figure 3.3 shows a chronological overview of each phase, including derived contributions and obtained publications. Figure 3.3 pretends to be self-contained in the sense that the citations represented by integers between brackets correspond to the enumerated bibliographical resources at the bottom of the figure.

In each phase of the development of this thesis, various methodological inputs have been used as described in Figure 3.2. Next, each of the four phases of development are described:

1. Initialisation. During this phase, existing software quality models and approaches for extending software quality models have been analysed, and an initial analysis of the state of the art in semantic technology quality specification have been performed. As a result, the initial version of the quality model for semantic technologies has been developed $\left(C_{4}\right)$. It is important to note that this phase took place during the Master studies, before the student enrolled in the Ph.D. program.

2. Development. During this phase, existing MCDM methods and a state of the art in the application of MCDM methods in the Software Engineering field have been analysed. As a result, the methodological contributions of this thesis as described in Section 3.2 have been developed, which include algorithms for automatic comparison of alternatives $(C 1)$ and methods for the extraction of the ANP and the AHP models (C2). Furthermore, in this phase the initial version of the quality model for semantic technologies developed in the previous phase has been improved and extended $\left(C_{4}\right)$ and an application of the RIDER framework in the semantic technology domain (C5) has been defined.

The results of this phase have been combined into RIDER, a Multiple Criteria Decision Making framework that extends the Analytic Network Process and that takes advantage of evaluation results of alternatives and of user quality requirements over such alternatives, and into an application of RIDER in the Semantic Web field for semantic technology recommendation.

3. Implementation. During this phase, existing software libraries have been used in order to provide the technological contributions of this thesis, which include software support for the RIDER framework (C3) and a web application for the recommendation of semantic technologies $(C 5)$.

4. Refinement. During this phase, continuous refinements and improvements have been performed over the main contributions of this thesis. 


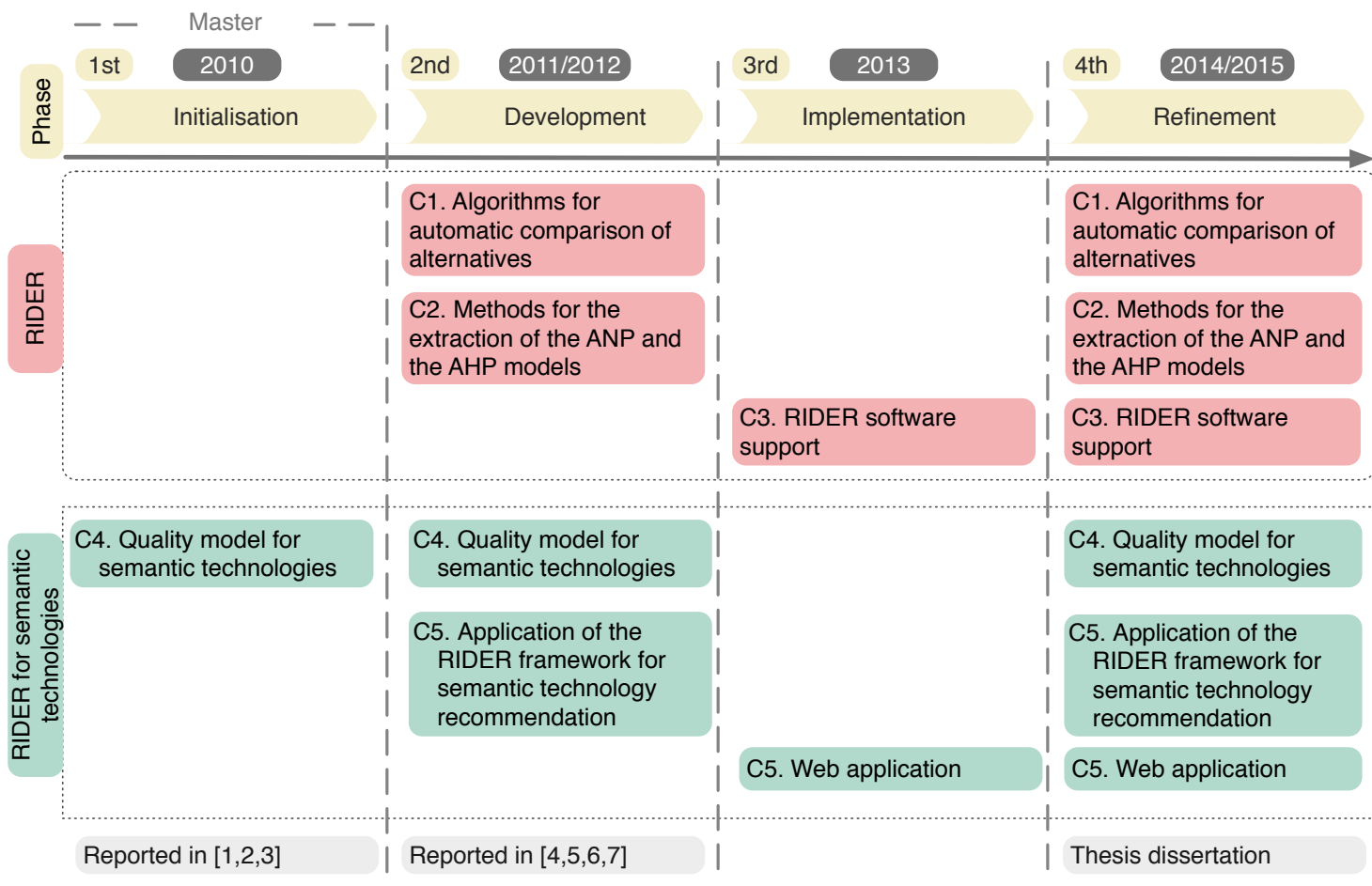

[1] F. Radulovic. A Software Quality Model for the Evaluation of Semantic Technologies. Master's thesis, Facultad de Informática (Universidad Politécnica de Madrid), 2011.

[2] F. Radulovic and R. García-Castro. Towards A Quality Model For Semantic Technologies. Software Quality Workshop 2011, within the International Conference on Computational Sciences and Its Applications (ICCSA2011). Santander, Spain. June, 2011. pp. 244-256

[3] F. Radulovic and R. García-Castro. Extending Software Quality Models - A Sample In The Domain of Semantic Technologies. In Proceedings of the 23rd International Conference on Software Engineering and Knowledge Engineering (SEKE2011). Miami, USA. July, 2011. pp. 25-30

[4] F. Radulovic and R. García-Castro. Semantic Technology Recommendation Based on The Analytic Network Process. In Proceedings of the 24th International Conference on Software Engineering and Knowledge Engineering (SEKE2012). Redwood City, California, USA. July, 2012. pp. 611-616

[5] F. Radulovic and R. García-Castro. An ANP Based Recommendation Framework with an Application in the Semantic Technology Domain. In Proceedings of the 26th European Conference on Operational Research (EURO2013). Rome, Italy. July, 2013

[6] F. Radulovic. A Recommendation Framework Based on the Analytic Network Process and its Application in the Semantic Technology Domain. In Proceedings of the 15th Conference of the Spanish Association for Artificial Intelligence (CAEPIA2013), Doctoral Consortium. Madrid, Spain. September, 2013

[7] F. Radulovic, R. García-Castro and A. Gómez-Pérez. SemQuaRE - An extension of the SQuaRE quality model for the evaluation of semantic technologies. Computer Standards \& Interfaces. Volume 38. February, 2015. pp. 101-112

Figure 3.3: Phases of the thesis development. 



\section{Chapter 4}

\section{The RIDER Recommendation Framework}

This chapter presents RIDER, a domain-independent and modular MCDM recommendation framework based on the AHP and the ANP methods that takes into account evaluation results of alternatives ${ }^{1}$ and user quality requirements defined over such alternatives, together with artefacts needed for its instantiation and a typical use case. RIDER improves the general AHP and ANP MCDM recommendation framework presented in Section 2.1.5 and brings solutions to the open research and engineering problems stated in Section 3.1 in several ways. First, RIDER provides domain-independent comparison algorithms for the automatic comparison of alternatives according to their evaluation results, as well as an extension of these algorithms for taking into account user quality requirements. Second, it provides a set of methods for the dynamic extraction of the AHP and the ANP models depending on user quality requirements.

RIDER consists of several separate components that can be exploited independently one from another:

- Quality model (Section 4.1). Quality models provide guidance in evaluations of alternatives and are a valuable resource for obtaining evaluation results. Furthermore, quality models can be useful as a reference for user quality requirements specification and for the definition of the AHP and ANP models. RIDER also provides an ontological representation for the quality model component.

- Evaluation results of alternatives (Section 4.2). In some domains, evaluation results of alternatives are available and these results are used in RIDER for the

\footnotetext{
${ }^{1}$ An alternative represents a concrete product, service or action to be recommended in a particular domain.
} 
comparison of alternatives. RIDER also provides an ontological representation for the evaluation results component.

- Comparison algorithms (Section 4.3) and the extensions of these algorithms that make use of user quality requirements (Section 4.4). In RIDER, evaluation results and user quality requirements are exploited by using algorithms for the automatic comparison of alternatives.

- A set of methods for extracting the AHP and the ANP models (Section 4.5). Another mechanism for exploiting user quality requirements in RIDER is through the definition of a model specifically related to a particular set of user quality requirements. This is achieved by extracting such model from the initial model of a domain that contains all domain-related criteria, using the RIDER network extraction methods.

- Pairwise comparisons of criteria (Section 4.6). In RIDER, pairwise comparisons are performed for the initial model of a domain that contains all domain-related criteria. These comparisons are used for deriving priorities between criteria in the models related to specific sets of user quality requirements, which are extracted using model extraction methods.

Figure 4.1 shows an overview of the RIDER framework with the previously explained components. The processes are represented with dash-lined rectangles while inputs and outputs in a process are represented with solid-lined rectangles.

RIDER is a generic recommendation framework independent of any particular domain. This means that RIDER can be instantiated in many different domains for delivering recommendations. The artefacts needed for the instantiation of RIDER and the workflow of a typical use case of using RIDER in a particular domain are described in Section 4.7.

RIDER has been implemented in Java in order to provide software support for the framework. This support consists of a Java library that provides RIDER functionalities and of a generic web application, which are described in Section 4.8.

The RIDER framework has been instantiated in one concrete domain, which is the domain of semantic technologies. Chapters 5 and 6 describe this instantiation.

Next, each component of the RIDER framework is described in detail.

\subsection{Quality Model}

Quality models provide a common framework for quality specification and evaluation by specifying a consistent terminology for quality and by providing guidance for its 


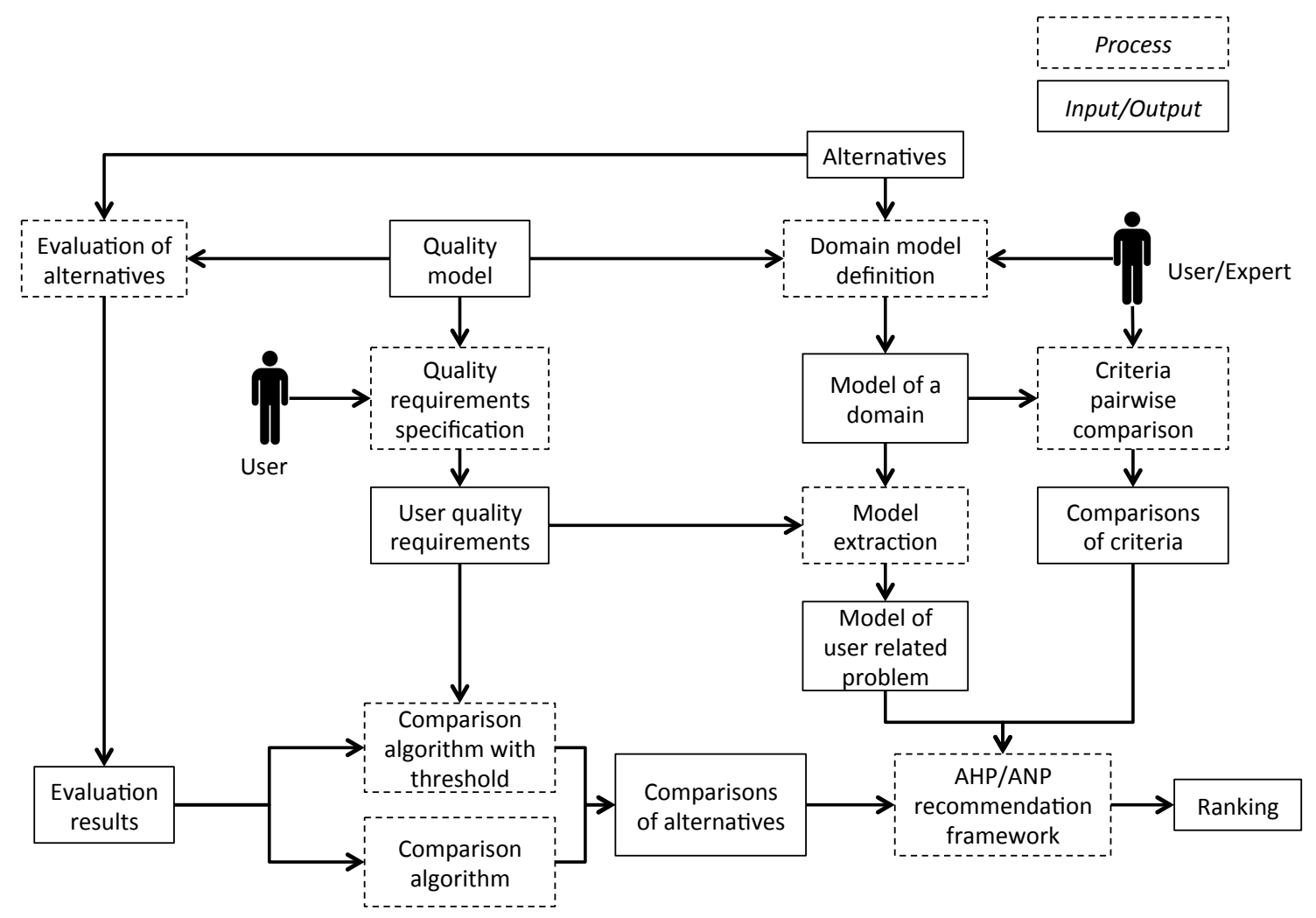

Figure 4.1: Overview of the RIDER framework.

measurement [8]. Furthermore, as seen in Section 2.5, quality models have been successfully used to date with MCDM methods.

Quality models are part of the RIDER recommendation framework (Figure 4.2) since they define the quality criteria to be used in the framework. Particularly, quality models are significant for:

1. Providing information about quality criteria that are useful in the creation of the AHP hierarchy or the ANP network for a particular domain. This information can be particularly significant because the identification of relevant criteria in a domain is identified as a resource-consuming task [104]. To this extent, instead of referring to an ordinary list of criteria, criteria can be combined and grouped in order to create different models.

2. Providing a reference for the evaluation of alternatives and for obtaining evaluation results.

3. Serving as a basis for the specification of user quality requirements. 


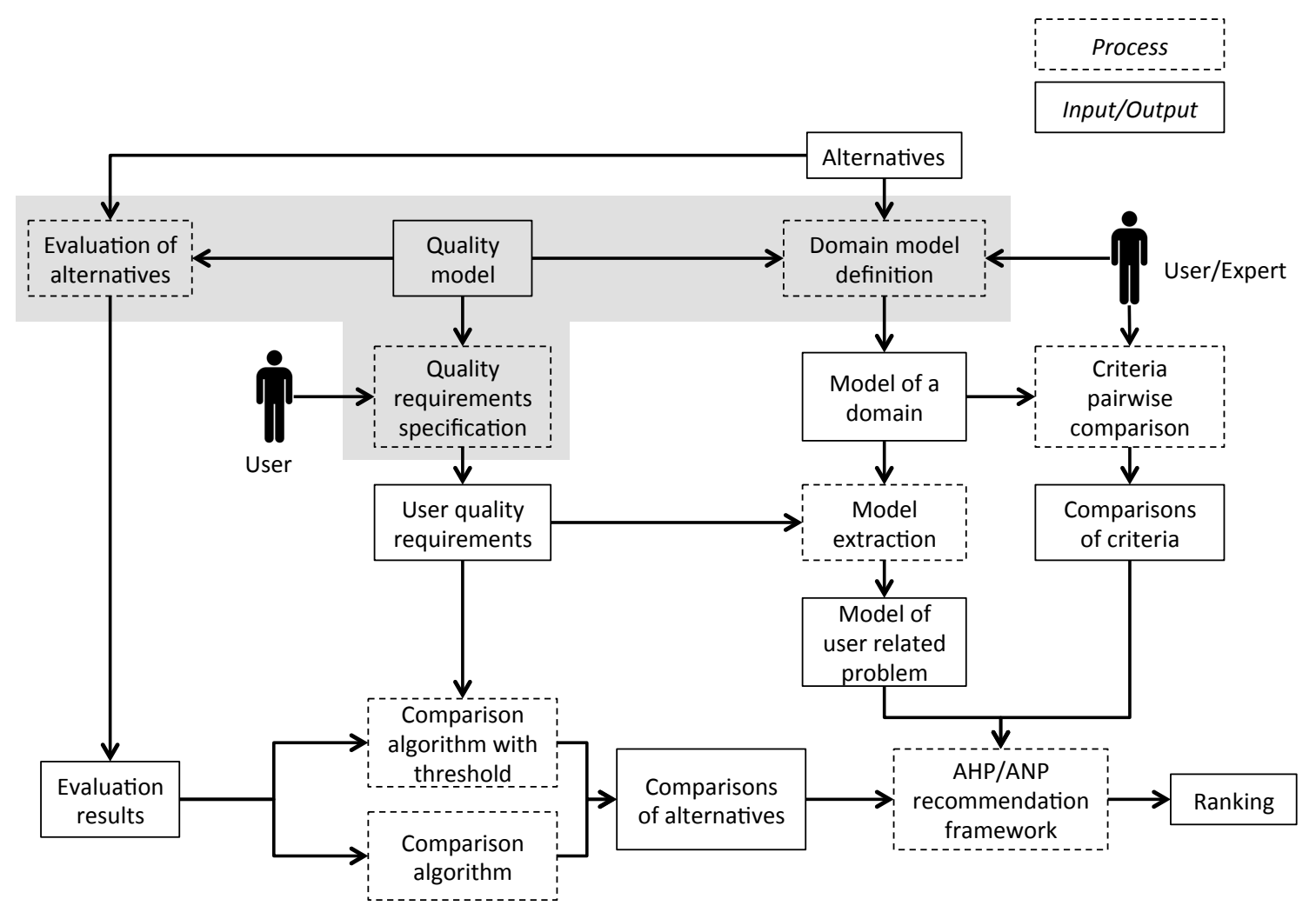

Figure 4.2: RIDER framework with the quality models component.

Regarding the first benefit, starting from a quality model, the corresponding AHP hierarchy of a domain can be defined in two consecutive steps:

1. Every indicator from the quality model becomes a leaf element of the criteria hierarchy. As seen in Section 2.1.1, in MCDM terminology those elements are called criteria.

2. Since every indicator is used for measuring a sub-characteristic, every subcharacteristic becomes a parent element of an indicator in a hierarchy.

In the case of the ANP, the ANP network of a domain can be defined in several consecutive steps:

1. Every indicator from the quality model becomes an element of the network, and those elements are related to criteria.

2. Since every indicator is used for measuring a sub-characteristic, the network elements are grouped into clusters, each containing those indicators that are 
related to a certain sub-characteristic. This way, each sub-characteristic becomes a cluster in a network.

3. Since a formula for the calculation of a measure (derived measure or indicator) can contain other measures as variables, the formulas imply that one measure is dependent on another (e.g., precision is used in the calculation of f-measure and, therefore, f-measure depends on precision). Therefore, based on formulas the dependencies between the indicators are deduced. Furthermore, dependencies between the quality indicators in a model can be defined based on expert opinions.

4. Every two dependent elements are connected with an arc; the element where the arc begins depends on the element where the arc ends.

5. Based on the dependencies between elements, dependencies between clusters are defined in such a way that two dependent elements imply a dependence between their clusters.

Regarding the second benefit, quality models provide the basis and instructions for obtaining evaluation results in a particular domain. The evaluation results that are obtained using a quality model can be useful when comparing alternatives in a recommendation process.

Regarding the third benefit, quality models provide the basis for the specification of user quality requirements since user quality requirements in a recommendation process are related to quality indicators in a quality model, i.e., to criteria in the AHP hierarchy or in the ANP network.

\section{Illustrative example.}

Let us consider as an example a part of the quality model for ontology matching tools. For measuring the Ontology alignment accuracy sub-characteristic, the quality model defines several quality indicators that can be used: Average alignment precision, Average alignment recall, Average alignment F-measure, and Average alignment $H$ measure. Because of this, these indicators are defined as elements in the Ontology alignment accuracy cluster of the ANP network (Figure 4.3).

The formulas for the F-measure and H-measure derived measures used for obtaining the indicators, shown below, suggest that these two measures depend on the precision and recall derived measures. Because of this, the Average alignment F-measure and Average alignment H-measure indicators are dependent on the Average alignment precision and Average alignment recall indicators. Furthermore, since $H$-measure is a special case of F-measure, Average alignment H-measure and Average alignment F-measure are also dependent on each other. Finally, because of these dependencies, the Ontology alignment accuracy cluster also depends on itself. 


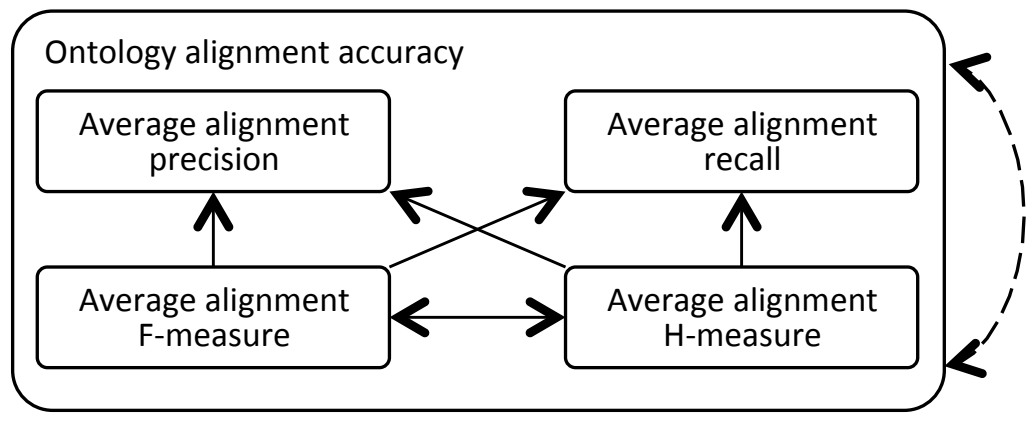

Figure 4.3: Sample subset of the semantic technology ANP network.

$$
\begin{gathered}
\text { F-measure }=\frac{\text { precision } \times \text { recall }}{(1-\alpha) \times \text { precision }+\alpha \times \text { recall }}, \alpha=[0 . .1] \\
\text { H-measure }=\frac{\text { precision } \times \text { recall }}{\text { precision }+ \text { recall }} \times 2
\end{gathered}
$$

\subsubsection{Quality Model Ontology}

The Quality Model ontology defines the vocabulary for representing quality models and their resources in any particular domain, and is based on the state of the art in quality models (Section 2.2). The Quality Model ontology has been published online ${ }^{2}$, and further development of the ontology is available for any interested parties ${ }^{3}$.

Figure 4.4 shows the graphical representation of the Quality Model ontology. The QualityMeasure class represents the quality measure, which can be either a BaseMeasure, a DerivedMeasure, or a QualityIndicator (see Section 2.2.5). One quality measure can be used to obtain some other quality measure (isUsedToObtain), and two quality measures can be in one of several types of relationships (collaboratesWith, dependsOn, or damages, see Section 2.2.6). Furthermore, a quality measure measures a quality characteristic, represented by the QualityCharacteristic class, and the two classes are connected with the measuresCharacteristic and isMeasuredWith properties.

The Quality Model ontology reuses the Ontology of units of Measure and related concepts (om) [106]. A quality measure is described with a unit of measure (om:Unit_of_measure) and with a scale (om:Measurement_scale). There are several possible types of scales, each represented by a class in the $O M$ ontology: ratio scale, interval scale, nominal scale, and ordinal scale [125]. Furthermore, for each scale it is possible to describe a ranking function (RankingFunction), which denotes whether a

\footnotetext{
${ }^{2}$ http://purl.org/net/QualityModel\#

${ }^{3}$ https://github.com/filiprd/QualityModel
} 


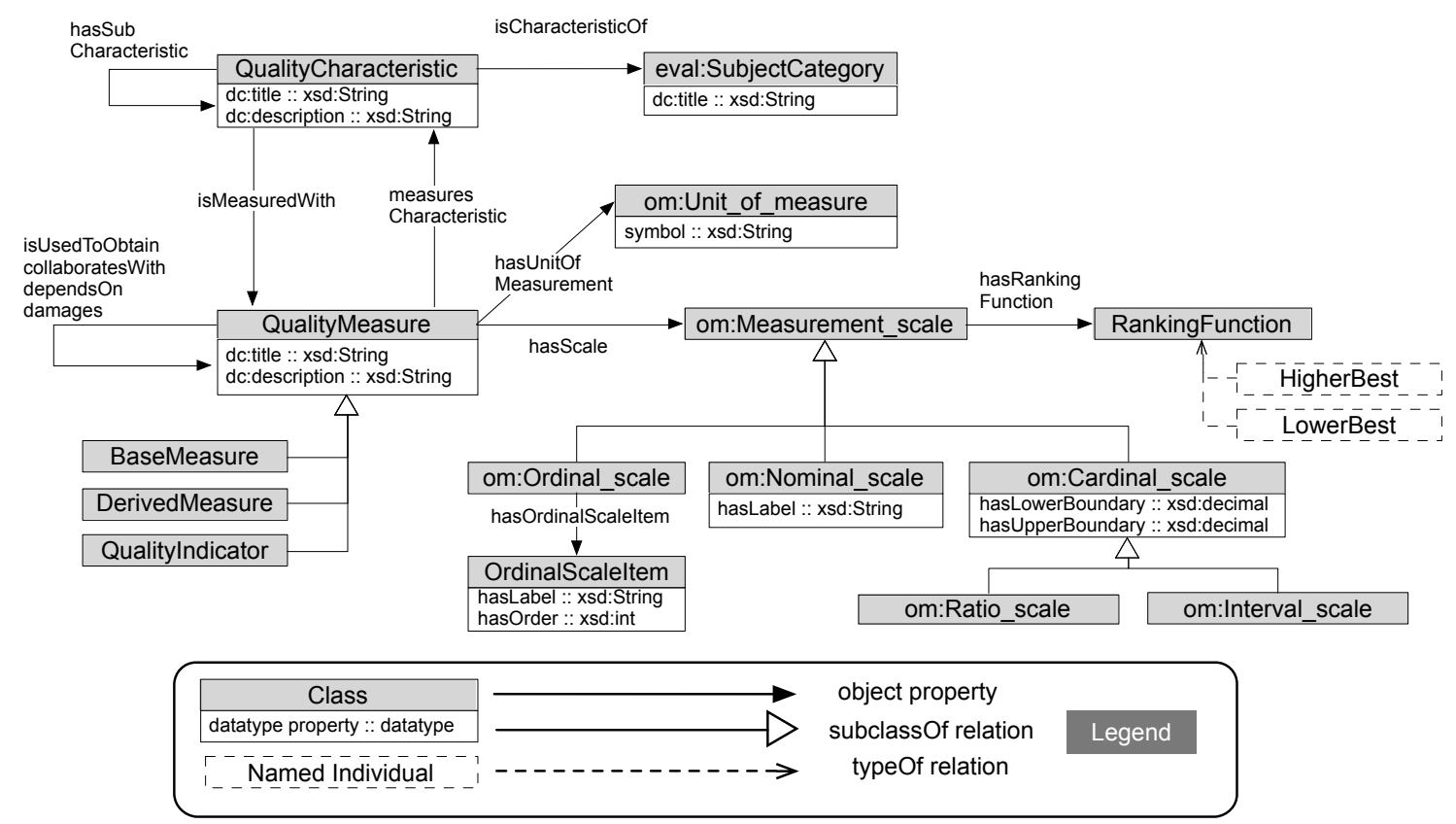

Figure 4.4: Graphical representation of the Quality Model ontology.

greater value on the scale is better or worse (HigherBest or LowerBest).

Finally, a quality characteristic can be related to a category of products, services or actions, which is represented by the eval:SubjectCategory class from the Evaluation Result ontology (Section 4.2.1). In the context of RIDER, a subject category represents a type of an alternative to be recommended.

The Quality Model ontology has been evaluated using the OOPS! pitfall scanner ${ }^{4}$ [101, 102]; the syntax of the ontology was also validated and the Pellet ${ }^{5}$ reasoner was used in order to evaluate the logical consistency of the ontology.

Listing 4.1 presents a short illustrative example of quality model data related to ontology matching tools. For the sake of simplicity, datatype declarations are omitted.

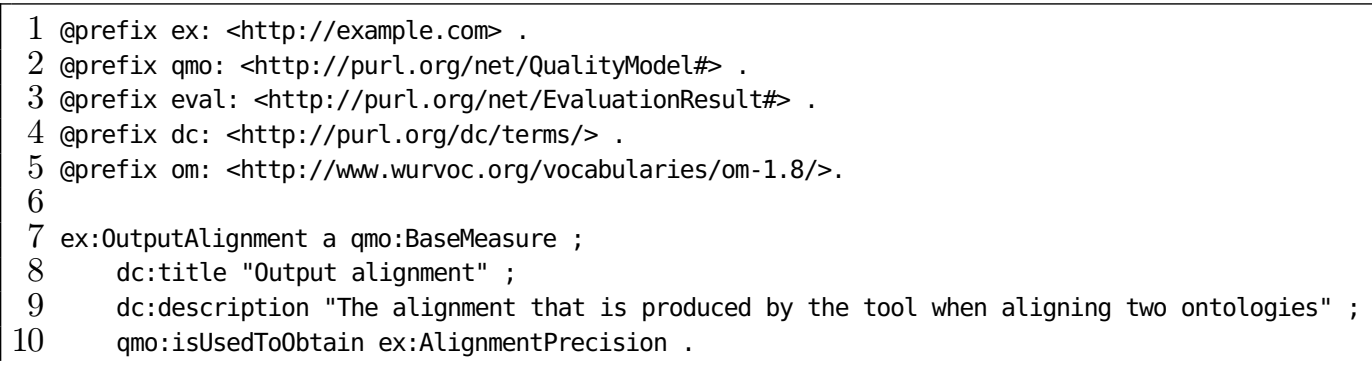

\footnotetext{
${ }^{4}$ http://oeg-upm.net/oops/

${ }^{5}$ http://clarkparsia.com/pellet/
} 


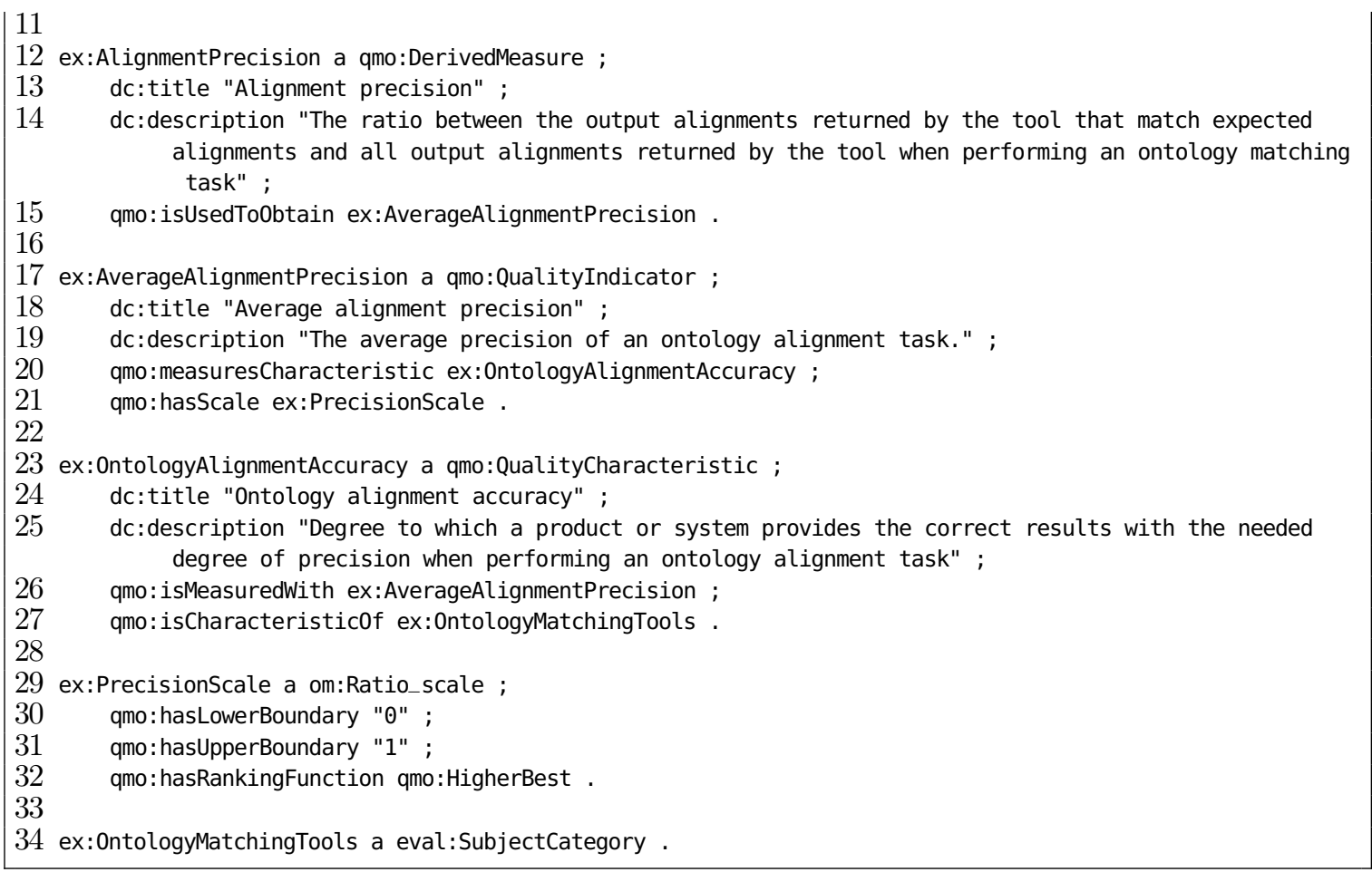

Listing 4.1: Example of using the Quality Model ontology for capturing data about quality models.

\subsection{Evaluation Results of Alternatives}

Evaluation processes are highly important, as discussed in Section 2.5. For example, according to ISO 9126, the evaluation of software is considered to be the integral part of the software development lifecycle [61]. To this date, a huge number of evaluations have been performed in many domains producing evaluation results of various alternatives. An example of this is the field of software engineering and the semantic technology domain (see Section 2.3). When evaluation results are available, it can be useful to base the comparison of alternatives in those results instead of relying on user or expert opinions and, because of this, evaluation results that are already available are part of the RIDER framework. Figure 4.5 shows the RIDER framework with the evaluation results component highlighted.

The values of evaluation results can belong to one of the four types of scale: nominal, ordinal, interval, or ratio [125]. The nominal scale is a type of scale in which results are descriptive labels with no significance of order. The ordinal, interval, and ratio scales are types of scale in which results can be ordered from best to worse, i.e., in which the best and worst values can be known. 


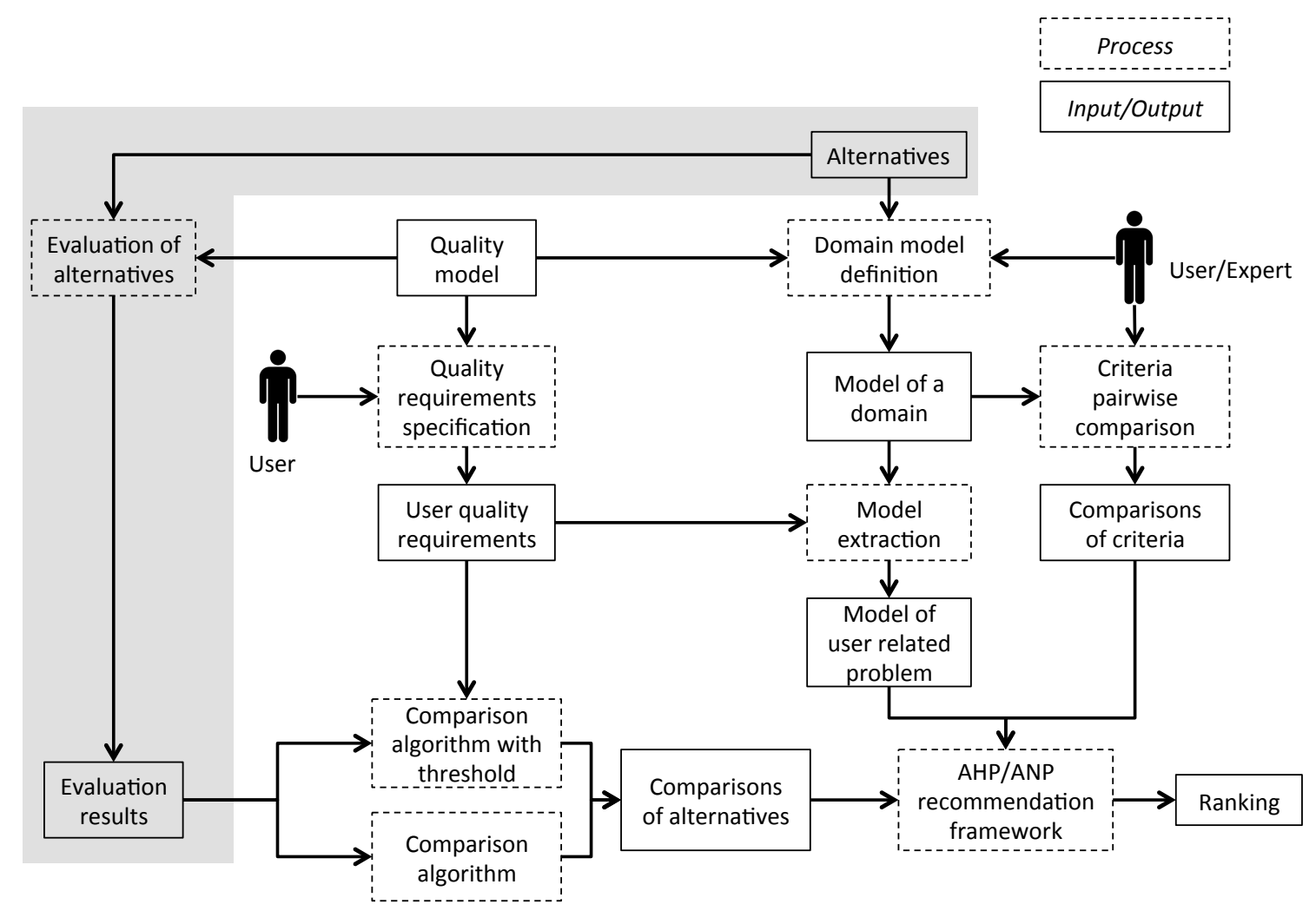

Figure 4.5: RIDER framework with the evaluation results component.

The difference between the ordinal, interval, and ratio scales is in the statistical analyses that can be applied to values on these scales [125]. The relevant characteristic of these three types of scale is that in some cases the lower and higher possible values are known (e.g., the age of a person can be from 0 to 99 years), and the distance between two values on any of these three scales can be precisely calculated. Additionally, depending on the semantics of the values, in some cases a higher value obtained in the evaluation denotes a better result (e.g., 0.8 in precision is better than 0.6 ), while in other cases a better result is denoted by a lower value (e.g., runtime of 2 seconds is better than the runtime of 6 seconds).

\subsubsection{Evaluation Result Ontology}

The Evaluation Result ontology defines the vocabulary for representing results obtained in an evaluation process; it extends the Quality Model ontology (QMO) (Section 4.1.1). The Evaluation Result ontology has been published online ${ }^{6}$, and

\footnotetext{
${ }^{6}$ http://purl.org/net/EvaluationResult\#
} 
Chapter 4. The RIDER Recommendation Framework

further development of the ontology is available for any interested parties ${ }^{7}$.

Figure 4.6 shows the graphical representation of the Evaluation Result ontology. The Evaluation class represents the process of evaluation, in which a particular alternative (i.e., product, service or action) is evaluated (EvaluationSubject class). Each evaluation produces a quality value (represented by the QualityValue class) which is related to a particular quality measure (qmo:QualityMeasure), and uses some input data (e.g., a document) which is represented by the EvaluationData class. Finally, each evaluation subject belongs to a certain category of products, services or actions, which is represented by the SubjectCategory class.

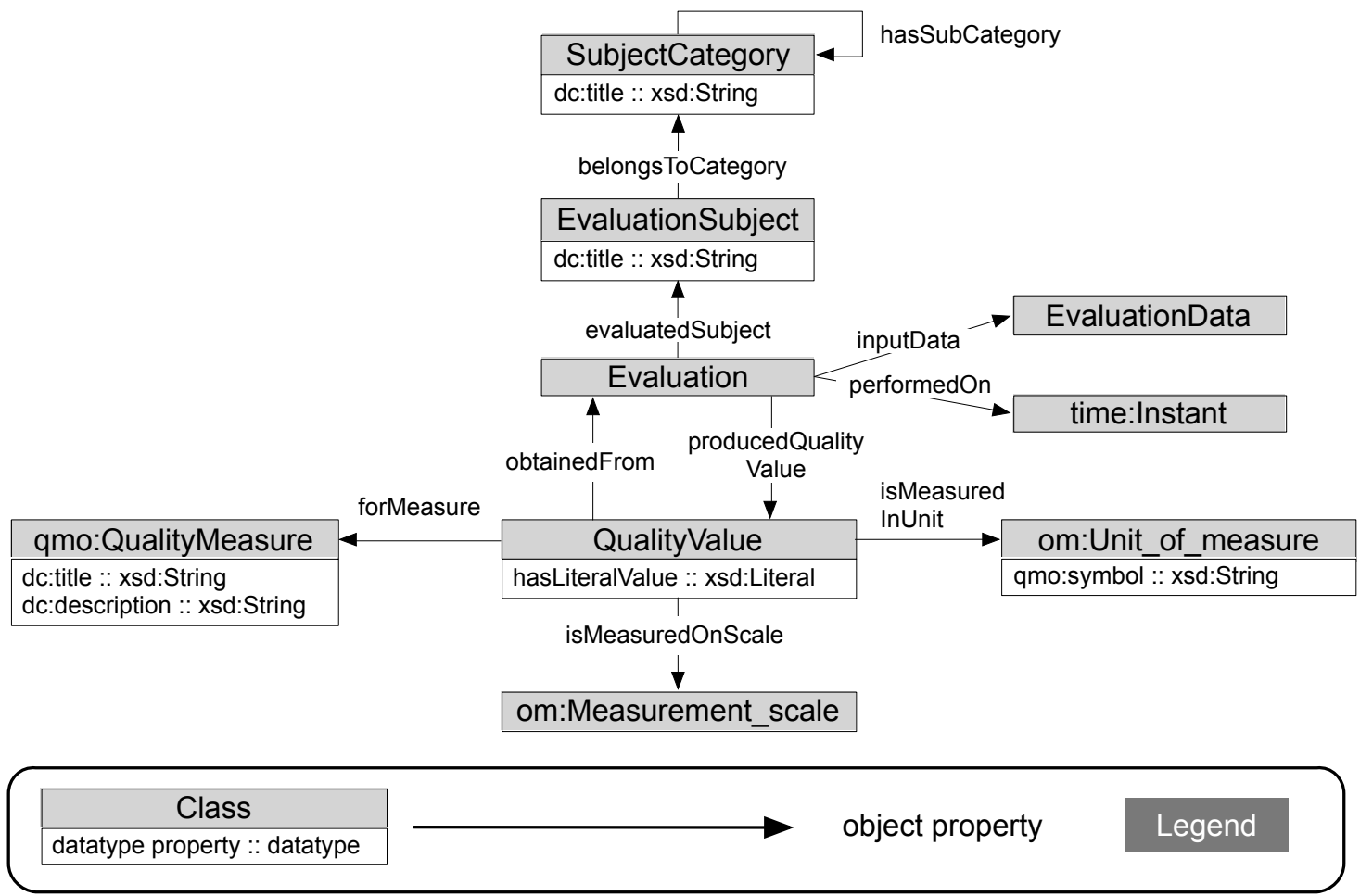

Figure 4.6: Graphical representation of the Evaluation Result ontology.

The Evaluation Result ontology reuses the Ontology of units of Measure and related concepts (om) and the W3C Time Ontology. Each evaluation is performed in a specific moment in time represented by the time:Instant class, and each quality value is expressed in a unit of measure (om:Unit_of_measure) and belongs to a particular scale (om:Measurement_scale), as defined in the Quality Model ontology.

Similar as with the Quality Model ontology, the Evaluation Result ontology has been evaluated using OOPS! and the syntax and logical consistency of the ontology

\footnotetext{
${ }^{7}$ https://github.com/filiprd/EvaluationResult
} 
have been checked.

Listing 4.2 presents a short illustrative example of evaluation result data related to one ontology matching tool $(\mathrm{YAM}++)$, which continues from the example in Listing 4.1. For the sake of simplicity, datatype declarations are omitted.

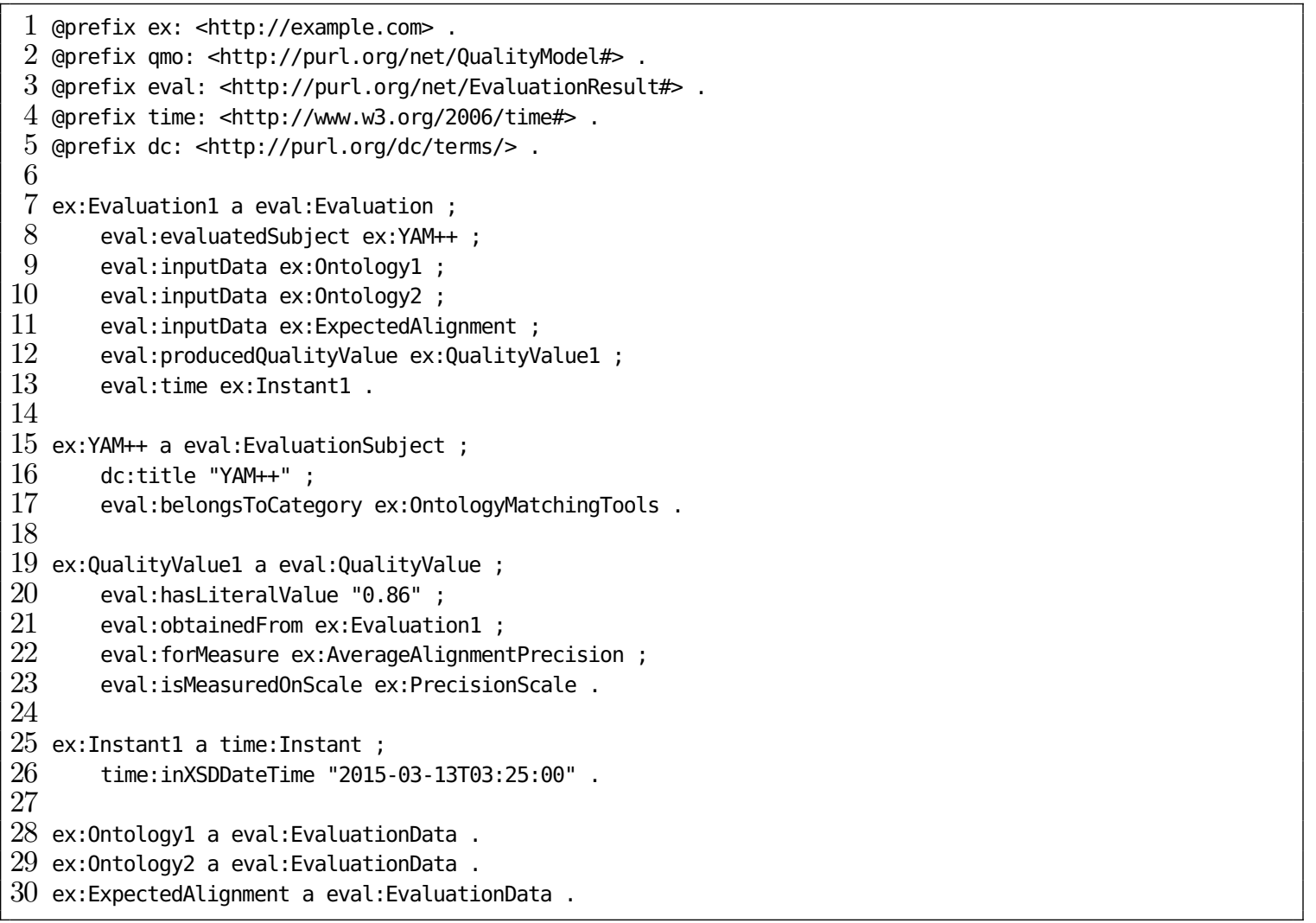

Listing 4.2: Example of using the Evaluation Result ontology for capturing data about quality evaluation results.

\subsection{Comparison Algorithm}

The RIDER recommendation framework is designed to exploit evaluation results of alternatives and to enable the automatic comparison of such alternatives with the goal of removing the need for user or expert opinions. In order to enable the automatic comparison of alternatives, a set of four comparison algorithms based on the standard Saaty's 1-9 comparison scale [118] has been defined. Apart from the automatic comparison of alternatives, these algorithms also help in exploiting alternative evaluation results and, hence, remove the need for expert or user opinions. Figure 4.7 shows the RIDER framework with the comparison algorithm component highlighted. 


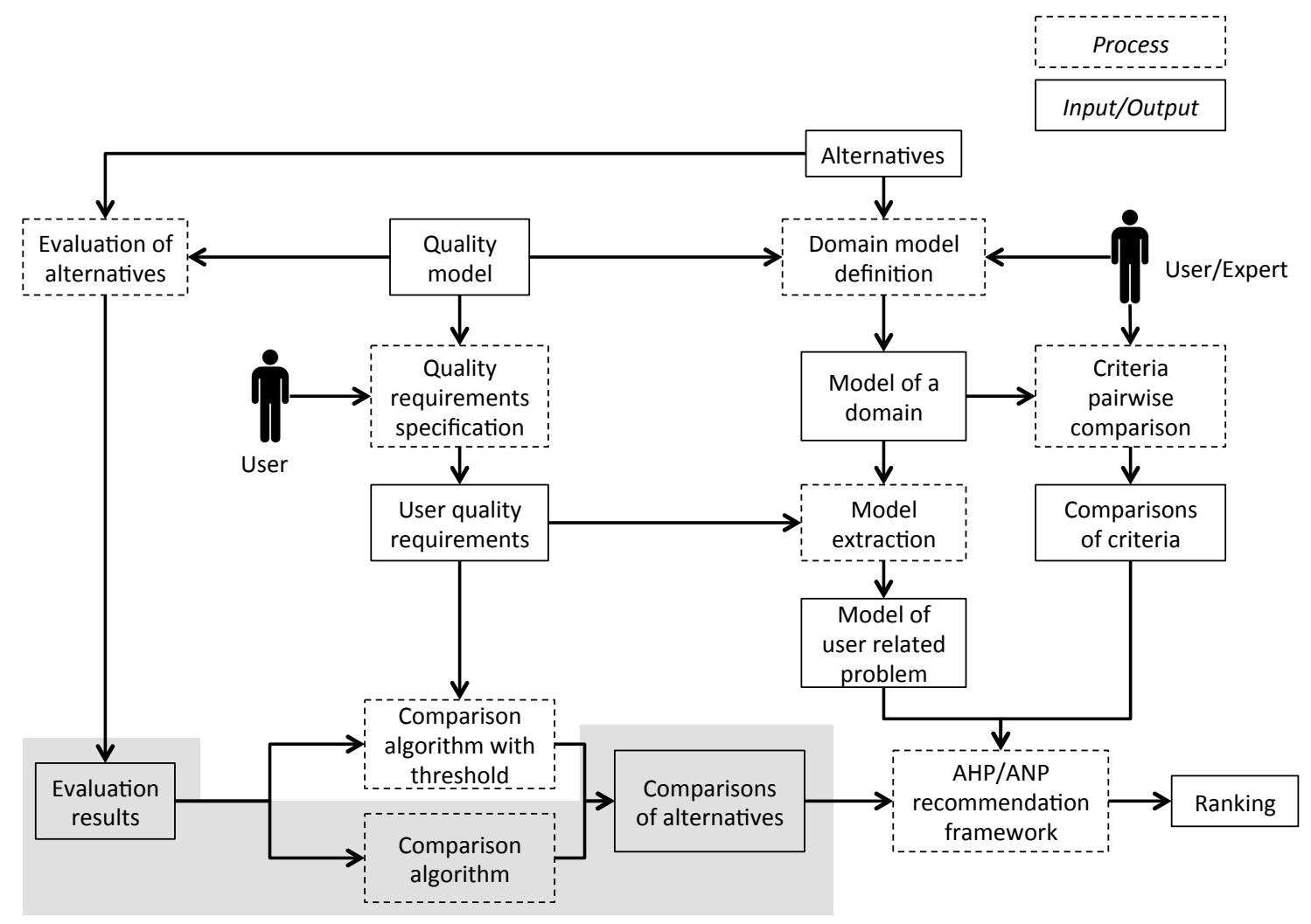

Figure 4.7: RIDER framework with the comparison algorithm component.

Comparison algorithms can be used to fill the values of a pairwise comparison matrix with alternative comparisons, i.e., with preferences of one alternative over another, after which the consistency of that matrix can be calculated independently of the comparison algorithm by using the consistency ratio proposed by Saaty (Section 2.1.4).

The comparison algorithm to be used in a specific case depends on the type of scale and on the availability of evaluation results (i.e., inputs), as shown in Table 4.1. In the general case, the inputs are evaluation results for two alternatives, result $r_{1}$ of alternative $A_{1}$ and result $r_{2}$ of alternative $A_{2}$, where both results belong to the same type of scale (Figure 4.8). The output is a number on the fundamental Saaty's comparison scale (Section 2.1.4) representing the preference (importance) of alternative $A_{1}$ over alternative $A_{2}$.

Algorithm 1 shows the general algorithm for filling the pairwise comparison matrix with alternative comparisons without threshold. This algorithm uses the comparison algorithms described further in this section (line 3) for deriving preferences between alternatives. In the reciprocal comparison matrix of size $n$, since the entries on the 
Table 4.1: Comparison algorithms depending on the type of scale and their inputs.

\begin{tabular}{|l|l|l|l|l|}
\hline Algorithm & $\begin{array}{l}\text { Nominal } \\
\text { scale }\end{array}$ & Simple & Scale distance & $\begin{array}{l}\text { Maximum dis- } \\
\text { tance }\end{array}$ \\
\hline Scale & Nominal & $\begin{array}{l}\text { Ordinal, } \\
\text { Interval or } \\
\text { Ratio }\end{array}$ & Interval or Ratio & Interval or Ratio \\
\hline Inputs & \multicolumn{2}{|c|}{$r_{1}, r_{2}$} & $\begin{array}{l}r_{1}, r_{2}, \text { min and } \\
\max \text { scale values }\end{array}$ & $\begin{array}{l}r_{1}, r_{2}, \text { all evalua- } \\
\text { tion results } V\end{array}$ \\
\hline
\end{tabular}

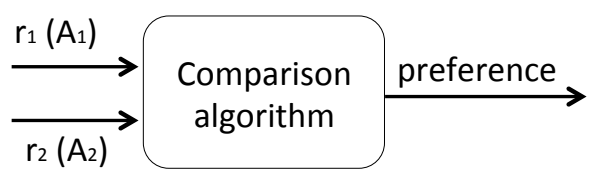

Figure 4.8: Inputs and outputs of the comparison algorithm without threshold.

main diagonal of the matrix are all equal to 1 , there are $n^{*}(n-1) / 2$ entries to be filled. Since all the matrices produced by this algorithm are by default reciprocal, the number of necessary comparisons of the general algorithm for filling a comparison matrix is $n^{*}(n-1) / \mathcal{L}$, where $n$ is the size of the matrix to be filled.

$\overline{\text { Algorithm } 1 \text { General algorithm for filling a pairwise comparison matrix without }}$ threshold

Input: $v_{i} \in V$, a set of $n$ evaluation results for $n$ alternatives all belonging to the same nominal, ordinal, interval or ratio scale

Output: $M=\left[m_{i j}\right]$, a pairwise comparison matrix of size $n$

Complexity: $o\left(n^{2}\right)$

1: for $i=1$ to $n-1$ do

2: $\quad$ for $j=i+1$ to $n$ do

3: $\quad$ preference $\leftarrow$ compare $\left(v_{i}, v_{j}\right)$

4: $\quad m_{i j}=$ preference

5: $\quad m_{j i}=1 /$ preference

6: return $M$

Next, the terminology regarding comparison algorithms used in this section and in the rest of this paper is defined, and the particularities of each comparison algorithm are presented.

Definition 1. Given two evaluation results $r_{1}$ and $r_{2}, r_{1}$ is said to be better than $r_{2}\left(r_{1} \succ r_{2}\right)$ if and only if one of the following conditions is satisfied: 
1. $r_{1}$ and $r_{2}$ are numerical values that belong to interval or ratio scale in which a lower value is preferred, and $r_{1}<r_{2}$.

2. $r_{1}$ and $r_{2}$ are numerical values that belong to interval or ratio scale in which a higher value is preferred, and $r_{1}>r_{2}$.

3. $r_{1}$ and $r_{2}$ belong to an ordinal scale, and $r_{1}$ has higher order than $r_{2}$.

\section{Nominal Scale Algorithm}

The Nominal scale algorithm (Algorithm 2) can be applied in the case of evaluation results that belong to the nominal scale. In this scale, since there is no ordering between the results, there is no way of determining which result is better or more preferred. An example of this is the comparison of the programming language two browsers are written in, where it is not possible to determine which programming language is more preferable. Therefore, if the two results are different, as well as if the two results are the same, a value of 1 (equal importance) is assigned in the comparison (i.e., when comparing $A_{1}$ to $A_{2}$ )

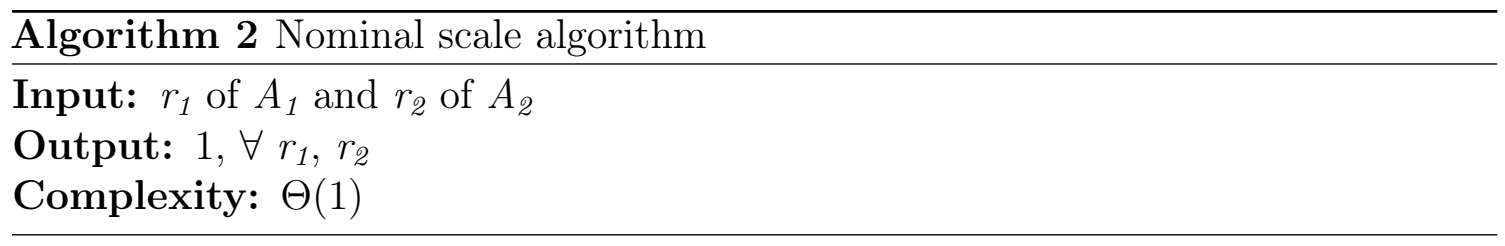

\section{Simple Algorithm}

The Simple algorithm (Algorithm 3) can be applied in the case of evaluation results that belong to the ordinal, interval, or ratio scales. In this algorithm, when comparing two alternatives (i.e., $A_{1}$ to $A_{2}$ ) there are three possible cases:

- If both results are equal, a value of 1 (equal importance) is assigned in the comparison (line 1).

- If the first result $\left(r_{1}\right)$ is better than the second result $\left(r_{2}\right)$, a value of 9 (extreme importance) is assigned in the comparison (line 3).

- If the second result is better than the first result, a value of $1 / 9$ is assigned in the comparison (line 4).

The Simple algorithm is particularly useful when the access to the complete set of evaluation results is restricted. On the other hand, this algorithm only distinguishes which alternative is better and, therefore, produces only two possible outputs. 


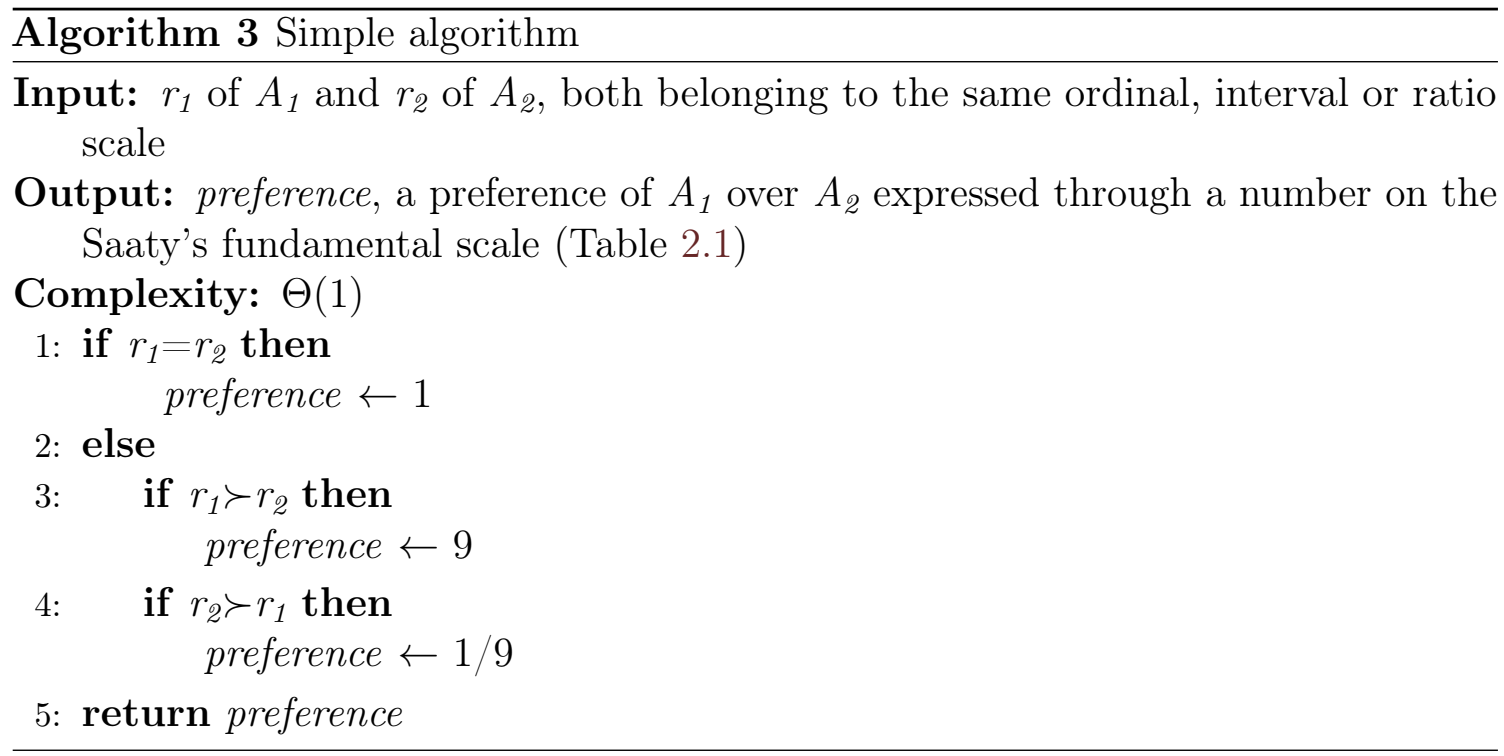

\section{Scale Distance Algorithm}

The Scale distance algorithm (Algorithm 4) can be applied in the cases of evaluation results that belong to the interval or ratio scales, and when the minimum and maximum values on a certain interval or ratio scale are available.

Therefore, the inputs of the algorithm are, besides $r_{1}$ and $r_{2}$, the minimum ( $m i n$ ) and maximum $(\max )$ possible values for the observed scale.

As there are nine possible outputs for the algorithm, there are also nine intervals from the lowest to the highest possible value on the scale, each interval denoting one algorithm output (Figure 4.9). The output of the algorithm is then determined by observing the interval to which the difference between the two results $\left(d_{r}\right)$ belongs. In the Scale distance algorithm, the intervals for each output are calculated based on the distance between the minimum ( $\min$ ) and maximum (max) possible values of the scale; i.e., the nine possible outputs correspond to nine intervals of the same length $(d)$ on the scale, and all the intervals cover the whole scale range. For example, the minimum value of 0 and maximum value of 90 on a scale imply the length of an interval of $d=10(90 / 9)$, and comparison of two results, $r_{1}=15$ and $r_{2}=42$, would imply the output of 3 (in the example it is assumed that a lower value is preferred), since the difference between the two results $\left(d_{r}=27\right)$ lies between the two and three intervals length.

This leads to three possible cases when comparing two alternatives (i.e., $A_{1}$ to $\left.A_{2}\right)$ :

- If both results are equal, a value of 1 is assigned in both comparisons (line 1).

- If the first result $\left(r_{1}\right)$ is better than the second result $\left(r_{2}\right)$, a value using formula 
Algorithm 4 Scale distance algorithm

Input: $r_{1}$ of $A_{1}$ and $r_{2}$ of $A_{2}$, both belonging to the same interval or ratio scale; min, minimum possible value on a scale; $\max$, maximum possible value on a scale

Output: preference, a preference of $A_{1}$ over $A_{2}$ expressed through a number on the Saaty's fundamental scale (Table 2.1)

Complexity: $\Theta(1)$

$$
\begin{aligned}
& \text { 1: if } r_{1}=r_{2} \text { then } \\
& \text { preference } \leftarrow 1 \\
& \text { 2: else } \\
& x \leftarrow \frac{\left|r_{1}-r_{2}\right|}{\frac{\max -\min }{9}}+0.5 \in[1,9] \in \mathbb{N} \\
& \text { 3: } \quad \text { if } r_{1} \succ r_{2} \text { then } \\
& \text { preference } \leftarrow x \\
& \text { preference } \leftarrow 1 / x
\end{aligned}
$$

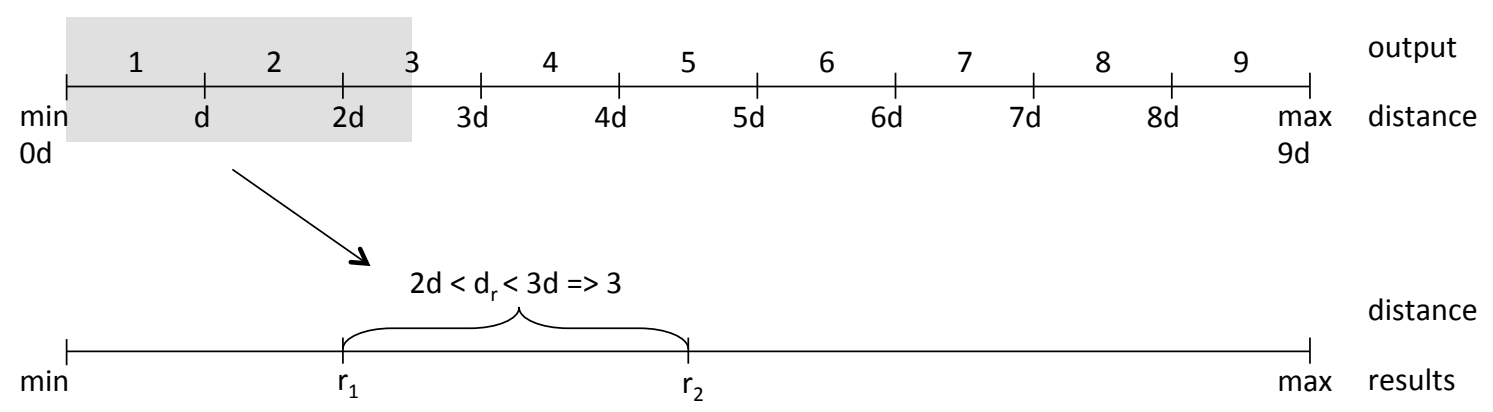

Figure 4.9: Scale distance algorithm visualization.

(4.1) is assigned when comparing the first alternative to the second one (line 3).

$$
\frac{\left|r_{1}-r_{2}\right|}{\frac{\max -\min }{9}}+0.5 \in[1,9] \in \mathbb{N}
$$

- If the second result is better than the first result, a reciprocal value of the one obtained using formula (4.1) is assigned when comparing the first alternative to the second one (line 4).

The Scale distance algorithm tends to produce outputs that cover more values on Saaty's scale and, hence, to provide more variations in the comparisons. However, it requires the minimum and maximum possible scale values to be known. 


\section{Maximum Distance Algorithm}

The Maximum distance algorithm (Algorithm 5) can be applied in the cases of evaluation results that belong to the interval or ratio scales and when all the evaluation results on a certain interval or ratio scale are available.

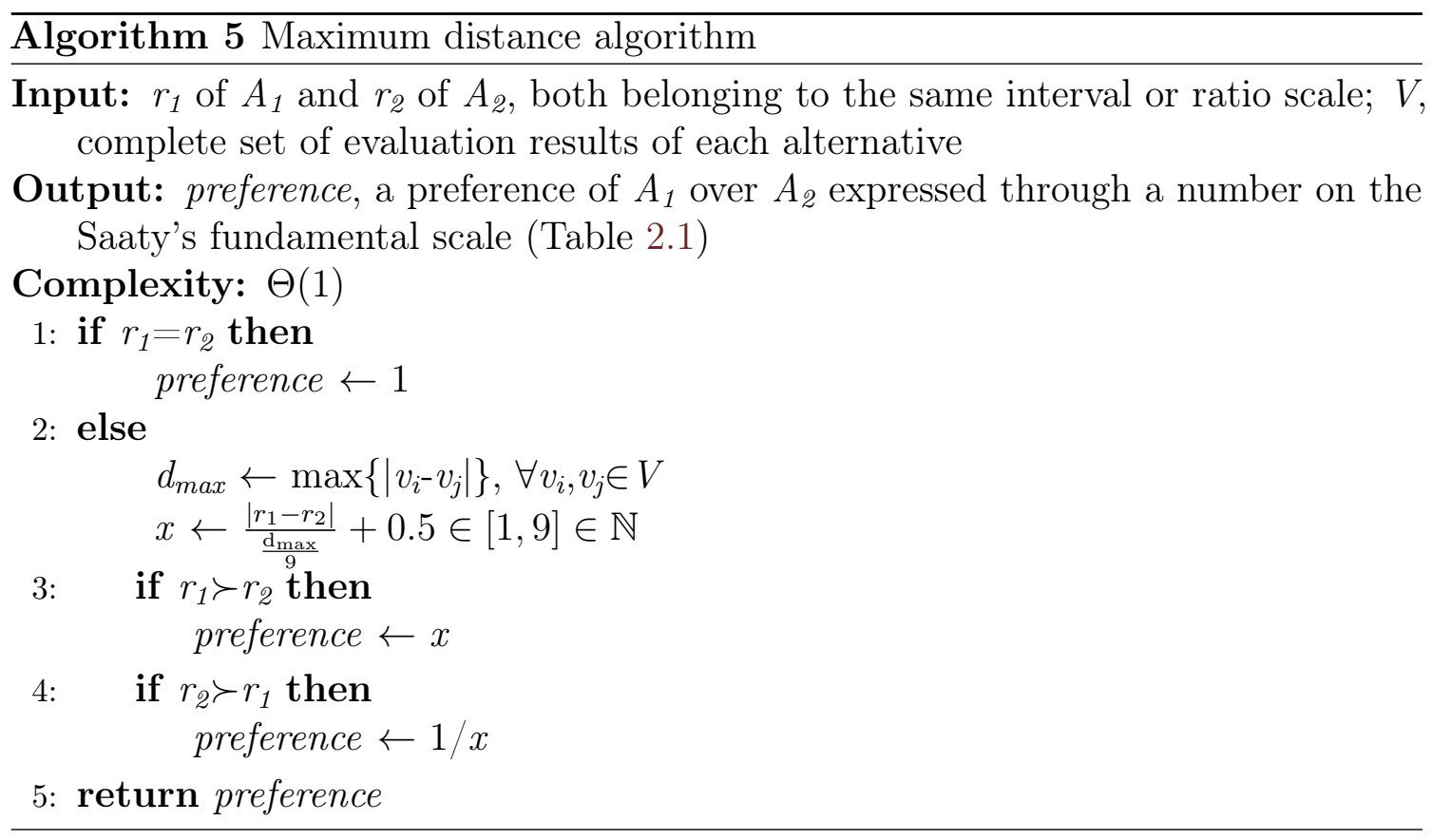

Therefore, the input of the algorithm is the complete set of evaluation results for all the alternatives, including $r_{1}$ and $r_{2}$.

Similarly as in the case of the Scale distance algorithm, the outputs of the Maximum distance algorithm are determined based on the output intervals (Figure 4.10). In the Maximum distance algorithm, however, the output intervals are calculated based on the maximum distance between any two evaluation results in a dataset $\left(d_{\max }\right)$, i.e., on a distance between the maximum $\left(r_{\max }\right)$ and minimum $\left(r_{\min }\right)$ values in evaluation results, and the output of the algorithm is then determined by observing the interval to which the difference between the two results $\left(d_{r}\right)$ belongs. This way, the nine possible outputs correspond to nine intervals of the same length on the scale, and all the intervals cover only the part of the scale to which the evaluation results belong.

This leads to three possible cases when comparing two alternatives (i.e., $A_{1}$ to $\left.A_{2}\right)$ :

- If both results are equal, a value of 1 is assigned in both comparisons (line 1). 


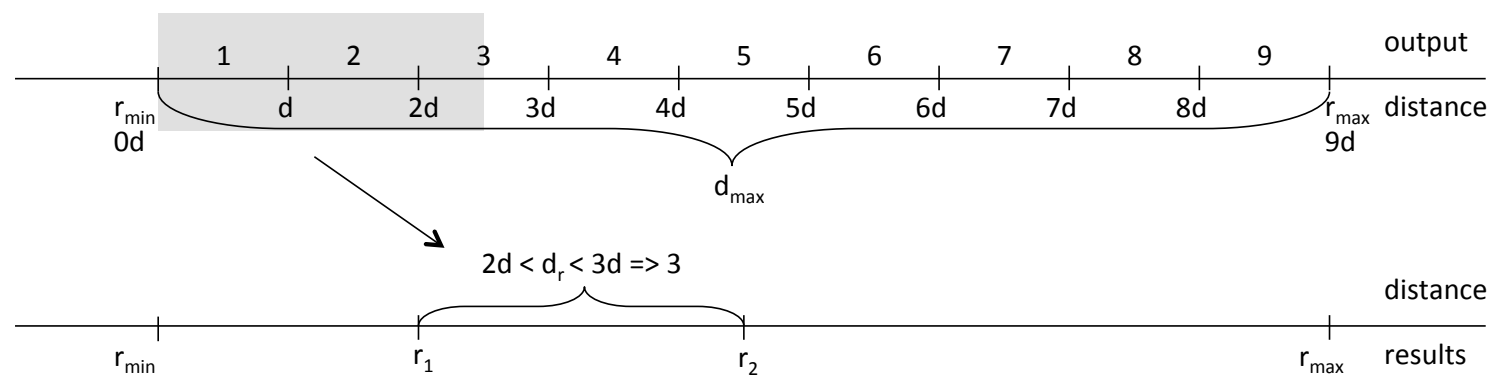

Figure 4.10: Maximum distance algorithm visualization.

- If the first result $\left(r_{1}\right)$ is better than the second result $\left(r_{2}\right)$, a value using formula (4.2) is assigned when comparing the first alternative to the second one (line 3 ).

$$
\frac{\left|r_{1}-r_{2}\right|}{\frac{\mathrm{d}_{\max }}{9}}+0.5 \in[1,9] \in \mathbb{N}
$$

- If the second result is better than the first result, a reciprocal value of the one obtained using formula (4.2) is assigned when comparing the first alternative to the second one (line 4 ).

Compared to the Scale distance algorithm, the Maximum distance algorithm tends to produce outputs that will cover all values on Saaty's scale and, hence, to provide a maximum degree of variation in the comparisons. However, this algorithm requires access to all evaluation results.

Illustrative example. In order to show an example, let us assume that we need to use the comparison algorithm to compare four alternatives $\left(A_{1}-A_{4}\right)$ with respect to a specific criterion (e.g., Ontology language component coverage, where a higher value denotes a better result) for which the following evaluation results are available: $86,82,70$, and 65 respectively.

Table 4.2 shows a comparison of the alternatives using the Maximum distance algorithm.

Table 4.2: An example of alternatives comparison using the Maximum distance algorithm.

\begin{tabular}{|c|c|c|c|c|c|}
\hline Alternatives & $A_{1}$ & $A_{2}$ & $A_{3}$ & $A_{4}$ & Importance \\
\hline$A_{1}$ & 1 & 2 & 7 & 9 & 0.527 \\
\hline$A_{2}$ & $1 / 2$ & 1 & 6 & 8 & 0.348 \\
\hline$A_{3}$ & $1 / 7$ & $1 / 6$ & 1 & 3 & 0.082 \\
\hline$A_{4}$ & $1 / 9$ & $1 / 8$ & $1 / 3$ & 1 & 0.041 \\
\hline
\end{tabular}


When comparing two alternatives, a value is assigned using formula 4.2. The difference between the highest and lowest evaluation result is $86-65=21$ and the interval between the results is divided into nine equal intervals of length $21 / 9=2.33$. When comparing $A_{1}$ to $A_{3}$, for example, the difference between the evaluation results of the observed alternatives is $86-70=16$, which implies that the output of the comparison is $7(16 / 2.33=6.86)$. In the case of comparing $A_{3}$ to $A_{4}$ the difference between the evaluation results of the observed alternatives is $70-65=5$, which implies that the output of the comparison is $3(5 / 2.33=2.14)$.

Finally, as previously explained, the consistency ratio of this comparison is calculated independently of the algorithms using the method proposed by Saaty (Section 2.1.4). The consistency ratio in this case is 0.04 , which implies that the comparison is consistent.

\subsection{User Quality Requirements}

It is often the case that when solving a specific problem not every characteristic is important [54]. For example, some users would prefer faster web browsers, while others would care more about resource utilization. Furthermore, for some of the characteristics users might have specific "good to have" quality requirements to be satisfied (e.g., the good memory utilization of a browser is less than $250 \mathrm{Mb}$ ).

The RIDER framework provides a possibility for a user to, if needed, specify user quality requirements; i.e., to specify criteria that are important for a particular problem that a user has to solve. User quality requirements are specified by users in the form of a criterion (i.e., a quality indicator of a product) and a threshold for that criterion (i.e., a "good to have" quality to be satisfied, or a concrete value in the case of the nominal scale). This approach is identical to other approaches for specifying quality requirements in the literature such as those of Jadhav and Sonar [67] and of Mochól [89]. These quality requirements are used as inputs in a recommendation process and no further inputs, such as alternatives comparison or model construction, are required from users.

As user quality requirements are an important information for the comparison of alternatives, the four comparison algorithms have been extended to take into account thresholds on criteria provided by users. Therefore, comparisons are adapted to user quality requirements by increasing the intensity of preference (importance) for those alternatives that satisfy them. Figure 4.11 shows the RIDER framework with the user quality requirements component highlighted.

Similarly as in the previous case, several possibilities are distinguished, depending on the type of scale and availability of evaluation results (Table 4.1). In the general case, the inputs are evaluation results for two alternatives, result $r_{1}$ of alternative $A_{1}$ and result $r_{2}$ of alternative $A_{2}$, and a threshold value $(t)$ from a user quality 


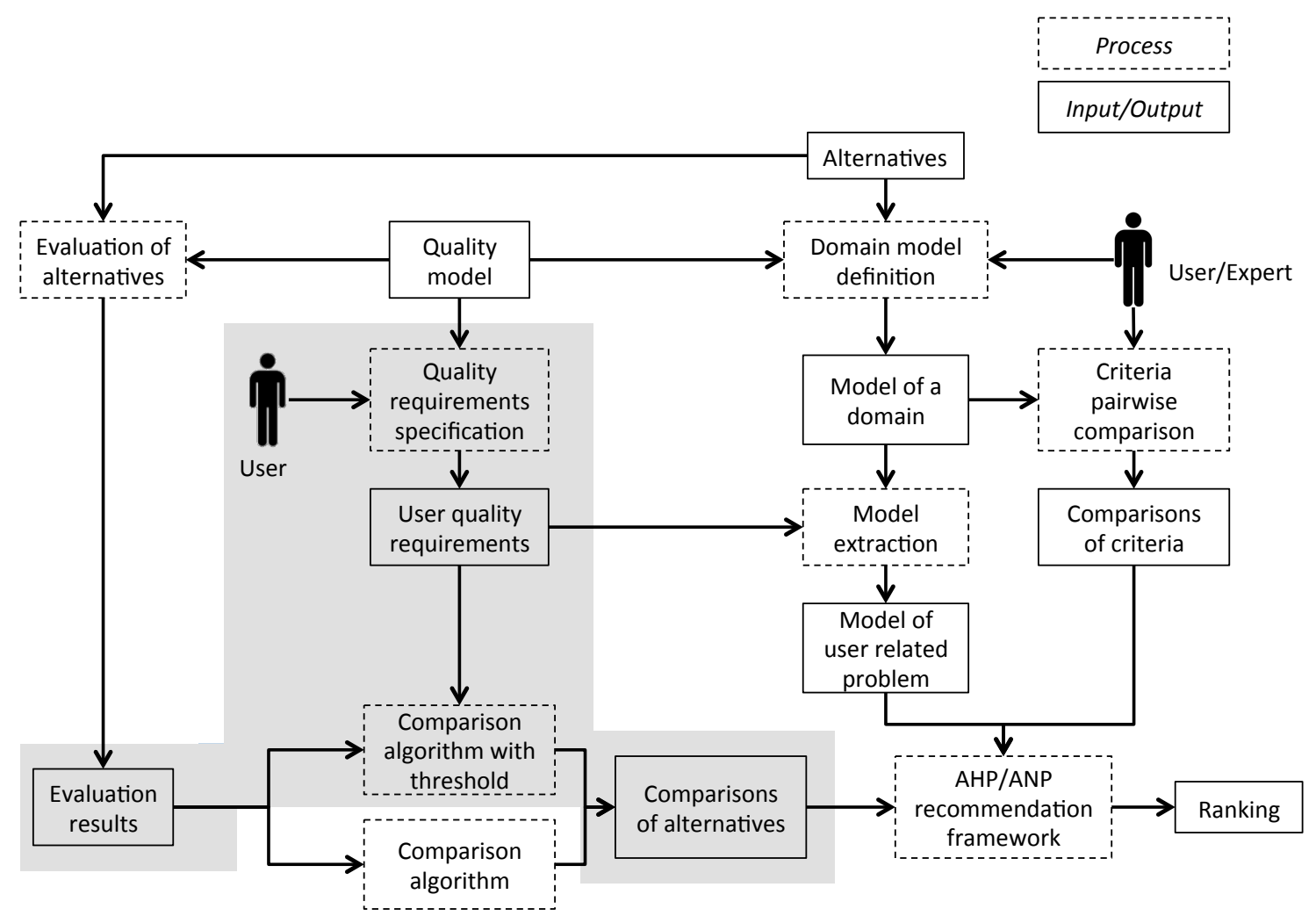

Figure 4.11: RIDER framework with the user quality requirements component.

requirement, where both results and the threshold belong to the same type of scale; the output is a number on standard Saaty's comparison scale (Figure 4.12) representing the preference (importance) of alternative $A_{1}$ over alternative $A_{2}$.

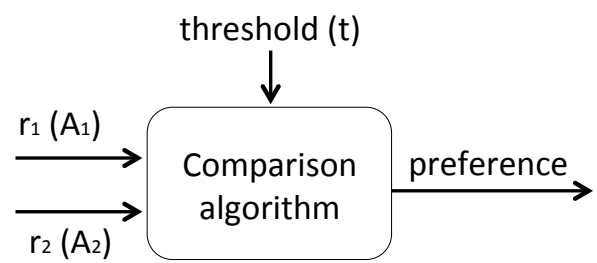

Figure 4.12: Inputs and outputs of the comparison algorithm with threshold.

Algorithm 6 shows the general algorithm for filling the comparison matrix with alternatives comparisons without threshold. This algorithm uses the comparison algorithms described further in this section (line 3) for deriving preferences between alternatives when a threshold from user quality requirements is introduced. Similarly as in the case of the algorithm without threshold, the number of necessary comparisons 


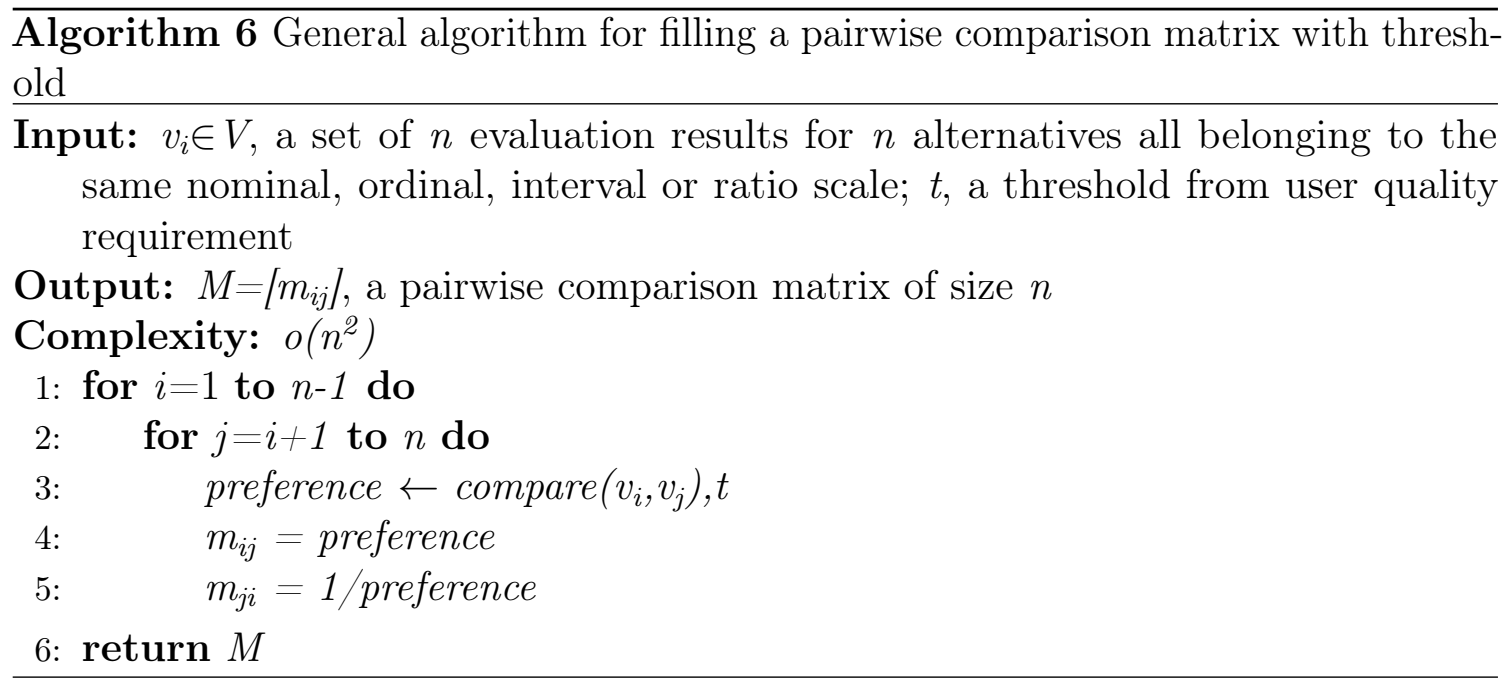

of the general algorithm for filling a comparison matrix is $n^{*}(n-1) / 2$, where $n$ is the size of the matrix to be filled.

Next, the terminology regarding comparison algorithms with thresholds is defined, and the particularities of each comparison algorithm when taking into account the threshold are presented.

Definition 2. Given an evaluation result $r$ and a threshold $t, r$ is said to satisfy threshold $t$ if and only if one of the following conditions is satisfied:

1. $r$ and $t$ belong to a nominal scale, and $r$ is equal to $t$.

2. $r$ and $t$ belong to an ordinal, interval or ratio scale, and $r$ is better than or equal to $t$, i.e., if $r \succeq t$.

\section{Nominal Scale Algorithm with Threshold}

The Nominal scale algorithm with threshold (Algorithm 7) can be applied in the case of evaluation results that belong to the nominal scale. In the case of the nominal scale, a threshold provided by the user is a piece of information which allows the comparison of different values. Therefore, there are three possible cases when comparing two alternatives (i.e., $A_{1}$ to $A_{2}$ ), depending on whether the evaluation results meet the threshold:

- If both results are equal among themselves (regardless whether they are different or the same as the threshold) or if both results are different among themselves and are also both different than the threshold (line 1), both alternatives are of equal importance. Therefore, a value of 1 (equal importance) is assigned in the comparison. 
Chapter 4. The RIDER Recommendation Framework

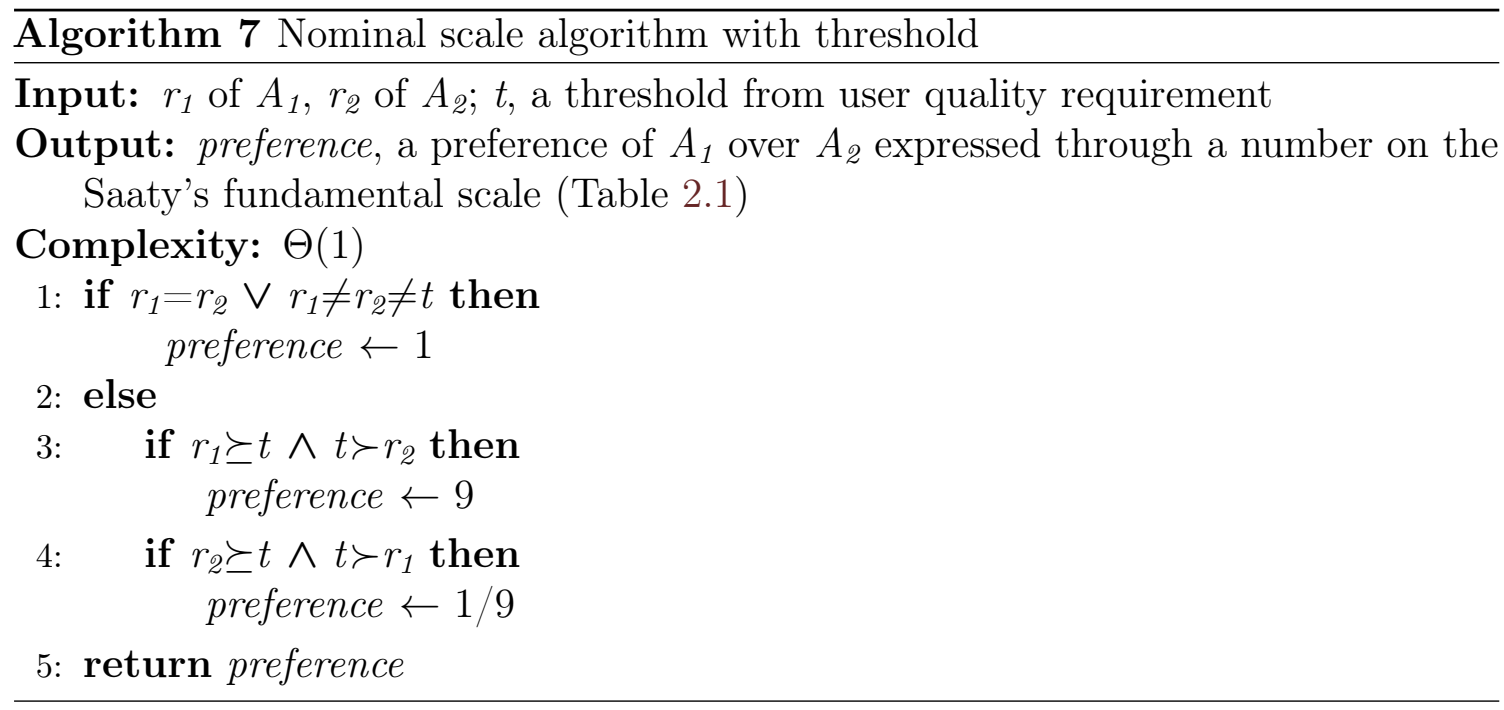

- If only the first result $\left(r_{1}\right)$ satisfies the threshold, a value of 9 (extreme importance) is assigned in the comparison (line 3).

- If only the second result $\left(r_{2}\right)$ satisfies the threshold, a value of $1 / 9$ is assigned in the comparison (line 4).

\section{Simple Algorithm with Threshold}

The Simple algorithm with threshold (Algorithm 8) can be applied in the case of evaluation results that belong to the ordinal, interval, or ratio scales. There are several possible cases when comparing two alternatives (i.e., $A_{1}$ to $A_{2}$ ), depending on whether or not evaluation results meet the threshold:

- If both results are equal, a value of 1 is assigned in the comparison (line 1).

- If both results satisfy the threshold, or none of them does (line 3), and if the first result $\left(r_{1}\right)$ is better than the second result $\left(r_{2}\right)$, a value from the Saaty's comparison scale has to be assigned to define preference; in this case, a value of 5 is chosen because it is in the middle of the Saaty's fundamental scale and divides the scale in two identical parts (line 4).

- If both results satisfy the threshold, or none of them does (line 3), and if the second result is better than the first result (line 5), a value of $1 / 5$ is assigned in the comparison.

- If only the first result satisfies the threshold (line 7), a value of 9 is assigned in the comparison. 


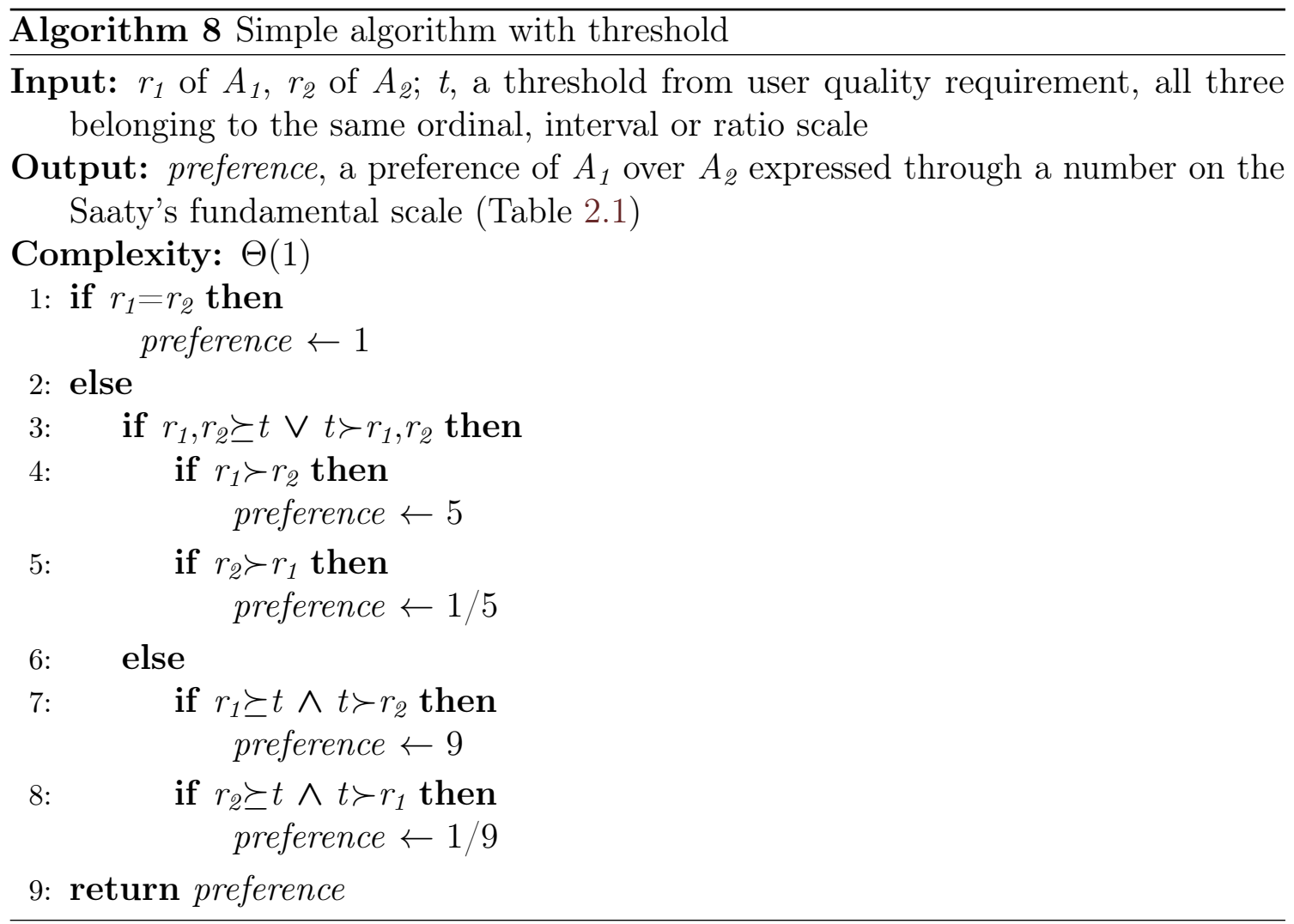

- If only the second result satisfies the threshold (line 8), a value of $1 / 9$ is assigned in the comparison.

\section{Scale Distance Algorithm with Threshold}

The Scale distance algorithm with threshold (Algorithm 9) can be applied in the cases of evaluation results that belong to the interval or ratio scales and when the minimum and maximum values on a certain interval or ratio scale are available.

There are several possible cases when comparing two alternatives (i.e., $A_{1}$ to $A_{2}$ ), depending on whether or not evaluation results meet the threshold:

- If both results are equal, a value of 1 is assigned in the comparison (line 1).

- If both results satisfy the threshold, or none of them does (line 3), and if the first result $\left(r_{1}\right)$ is better than the second result $\left(r_{2}\right)$, a value from formula (4.1) (Section 4.3) is assigned in the comparison (line 4).

- If both results satisfy the threshold, or none of them does (line 3), and if the second result is better than the first result (line 5), a reciprocal value of the one obtained using formula (4.1) is assigned in the comparison. 
Chapter 4. The RIDER Recommendation Framework

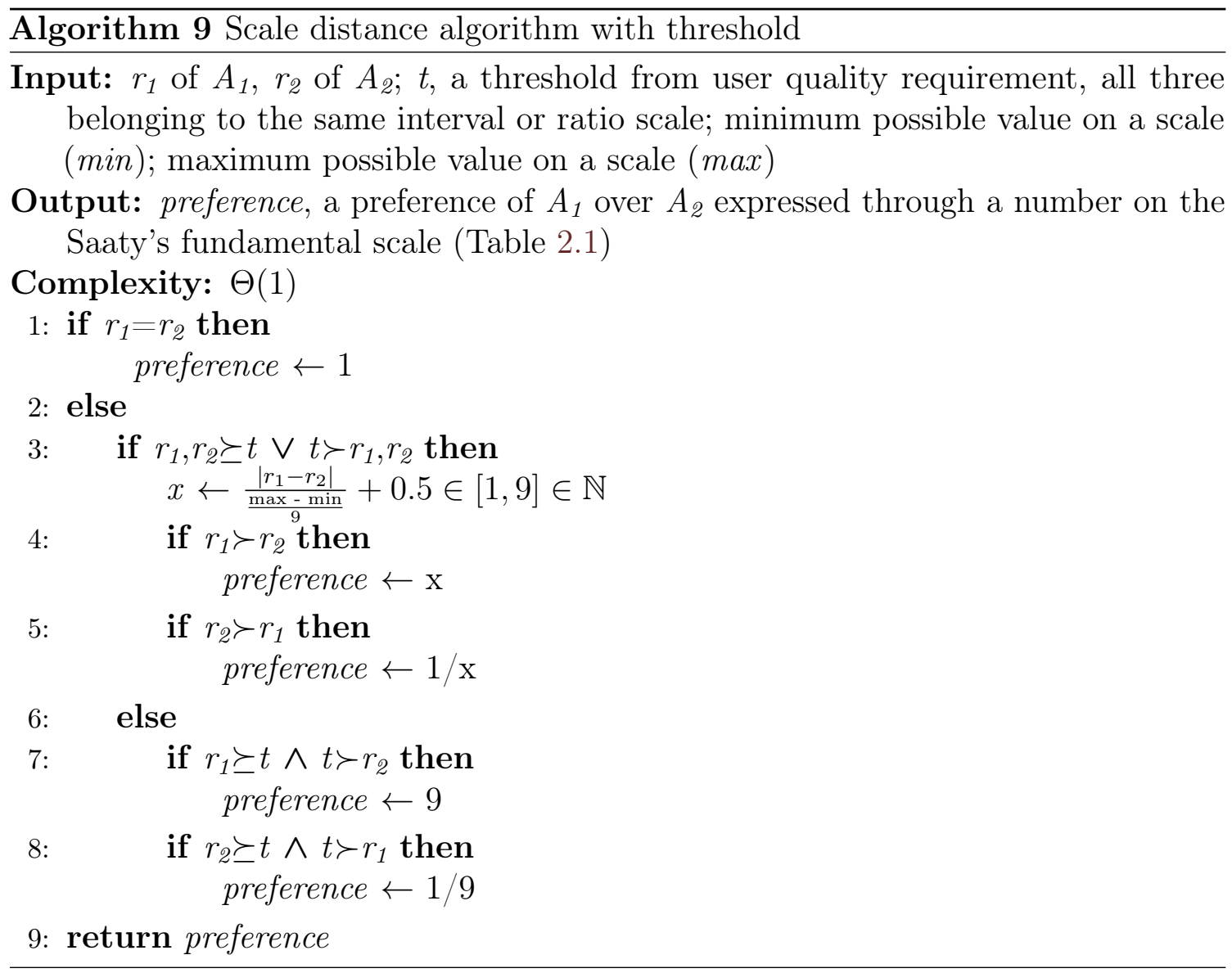

- If only the first result satisfies the threshold (line 7), a value of 9 is assigned in the comparison.

- If only the second result satisfies the threshold (line 8), a value of $1 / 9$ is assigned in the comparison.

\section{Maximum Distance Algorithm with Threshold}

The Maximum distance algorithm with threshold (Algorithm 10) can be applied in the cases of evaluation results that belong to the interval or ratio scales and when all the evaluation results on a certain interval or ratio scale are available.

There are several possible cases when comparing two alternatives (i.e., $A_{1}$ to $A_{2}$ ), depending on whether or not evaluation results meet the threshold:

- If both results are equal, a value of 1 is assigned in the comparison (line 1).

- If both results satisfy the threshold, or none of them does (line 3), and if the 


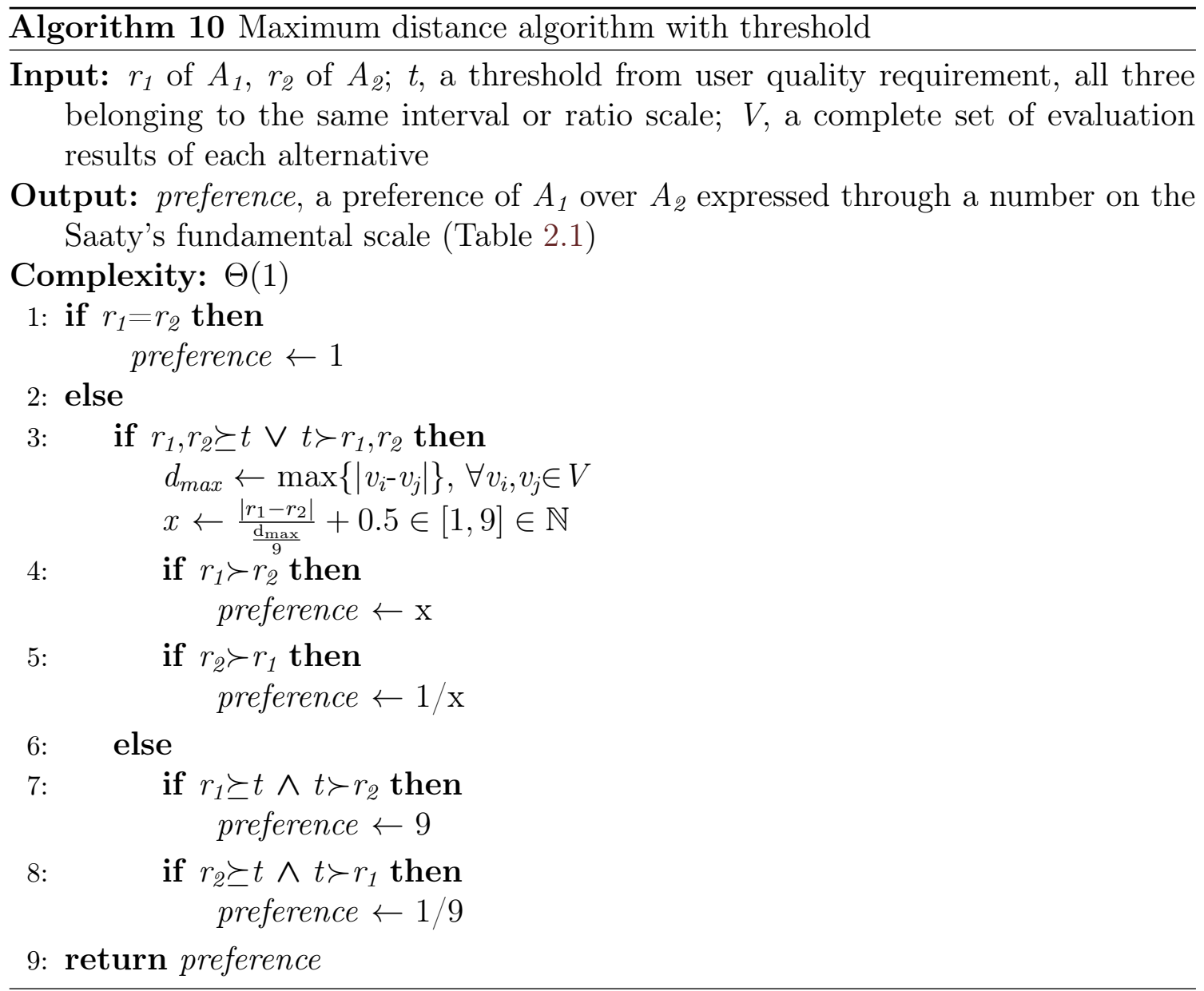

first result $\left(r_{1}\right)$ is better than the second result $\left(r_{2}\right)$, a value from formula (4.2) (Section 4.3) is assigned in the comparison (line 4).

- If both results satisfy the threshold, or none of them does (line 3), and if the second result is better than the first result (line 5), a reciprocal value of the one obtained using formula (4.2) is assigned in the comparison.

- If only the first result satisfies the threshold (line 7), a value of 9 is assigned in the comparison.

- If only the second result satisfies the threshold (line 8), a value of $1 / 9$ is assigned in the comparison.

Illustrative example. In order to show an example, let us refer to the example in the previous section in which four alternatives $\left(A_{1}-A_{4}\right)$ were compared with respect to a specific criterion (e.g., Ontology language component coverage, where a higher 
Chapter 4. The RIDER Recommendation Framework

value denotes a better result) for which the following evaluation results are available: $86,82,70$, and 65 respectively. Let us assume in this example that a user has specified a quality requirement of at least $75 \%$ of the ontology language components to be covered.

Table 4.3 shows a comparison of the alternatives using the Maximum distance algorithm with threshold.

Table 4.3: An example of alternatives comparison using the Maximum distance algorithm with threshold.

\begin{tabular}{|c|c|c|c|c|c|}
\hline Alternatives & $A_{1}$ & $A_{2}$ & $A_{3}$ & $A_{4}$ & Importance \\
\hline$A_{1}$ & 1 & 2 & 9 & 9 & 0.575 \\
\hline$A_{2}$ & $1 / 2$ & 1 & 9 & 9 & 0.328 \\
\hline$A_{3}$ & $1 / 9$ & $1 / 9$ & 1 & 3 & 0.056 \\
\hline$A_{4}$ & $1 / 9$ & $1 / 9$ & $1 / 2$ & 1 & 0.039 \\
\hline
\end{tabular}

In this example, two alternatives satisfy the quality requirement $\left(A_{1}\right.$ and $\left.A_{2}\right)$ while two do not satisfy it $\left(A_{3}\right.$ and $\left.A_{4}\right)$. Therefore, when comparing an alternative that satisfies the threshold to the alternative that does not satisfy it (i.e., when comparing $A_{1}$ or $A_{2}$ to $A_{3}$ or $A_{4}$ ), a value of 9 is assigned.

When comparing $A_{3}$ to $A_{4}$, which both do not satisfy the threshold, or $A_{1}$ to $A_{2}$, which both satisfy the threshold, a value is assigned using formula 4.2. The difference between the highest and lowest evaluation result is $86-65=21$ and the interval between the results is divided into nine equal intervals of length $21 / 9=2.33$. In the case of $A_{3}$ and $A_{4}$ the difference between the evaluation results of the observed alternatives is $70-65=5$, which implies that the output of the comparison is 3 . In the case of $A_{1}$ and $A_{2}$ the difference between the evaluation results of the observed alternatives is $86-82=4$, which implies that the output of the comparison is 2 .

Comparing with the example without threshold in the previous section, it can be observed that the intensity of preferences when comparing those alternatives that satisfy the threshold to those alternatives that do not satisfy it have increased. For example, when comparing $A_{1}$ to $A_{3}$, unlike in the previous example in which the value of 7 has been assigned, in this example a value of 9 has been assigned in the comparison.

Finally, the consistency ratio of this comparison is 0.08 , which implies that the comparison is consistent. 


\subsection{Model Extraction Methods}

The first step in the AHP and the ANP methods is to define the model of a recommendation problem [114], which includes all the identified alternatives and all the criteria with respect to which the alternatives can be compared.

Since the model can contain a large number of criteria, like for example in the case of simulation software [54], and since different users can have different quality requirements, not every criterion contributes equally to a user final decision (as argued in Section 3.1). For example, a user might want to use a fast web browser, but will not care about resource utilization. Therefore, although the initial model provides valuable information about the problem, different sub-models will be suitable to different users.

In this thesis, Model extraction is defined as a process in which, based on the user quality requirements, the initial model in a domain (i.e., the model that contains all the identified criteria) is transformed into a sub-model that contains only those criteria that are relevant for the specified quality requirements. In order to give more importance to user quality requirements in the recommendation process, there are several alternatives for the extraction of the AHP or the ANP model (i.e., hierarchy and network, respectively), depending on the criteria that are included into the model of the user's problem and on the criteria according to which the alternatives are compared to. These alternatives are formalised into model extraction methods; Figure 4.13 shows the RIDER framework with the model extraction method component highlighted.

The inputs for a model extraction method are a set of user quality requirements and the AHP or the ANP model of the domain in which the recommendation is going to be performed, including all the criteria and alternatives. The output is the model that is going to be used in the recommendation which, depending on the extraction method, can be a reduced model with an accent on user quality requirements.

After alternatives are added, with respect to the criteria that can appear in an AHP hierarchy, there are several possibilities; the AHP hierarchy can consist of:

a) Only those criteria that appear in the user quality requirements (Requirements hierarchy, Algorithm 11).

b) All the criteria defined in the initial AHP hierarchy of a domain, regardless of those appearing in the user quality requirements (Complete hierarchy, Algorithm $12)$.

After alternatives are added, with respect to the criteria that can appear in an ANP network, there are several possibilities; the ANP network can consist of:

a) Only those criteria that appear in the user quality requirements (Requirements network, Algorithm 13). 


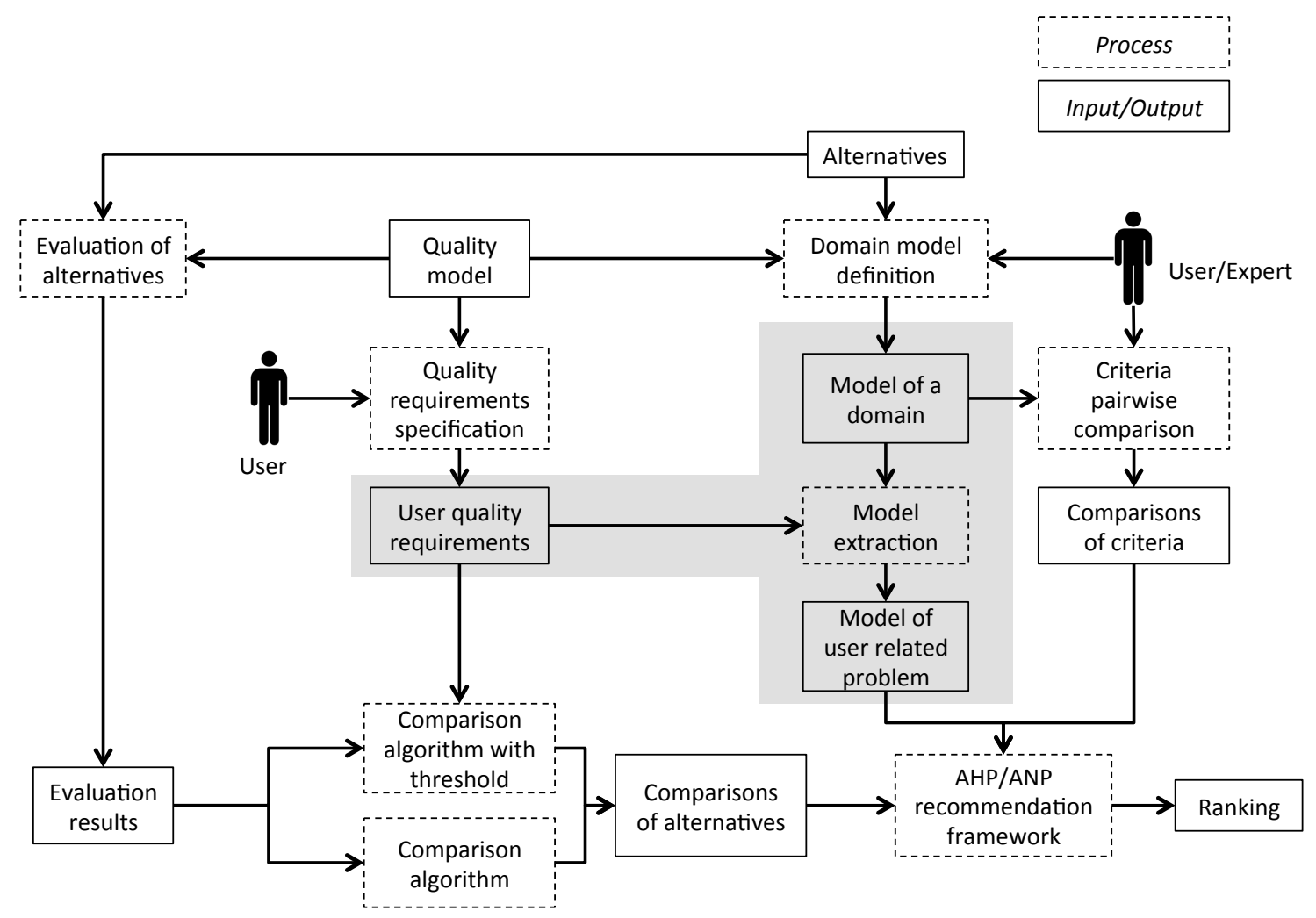

Figure 4.13: RIDER framework with the model extraction method component.

b) Those criteria that appear in the user quality requirements, together with the criteria from the initial ANP network that influence the ones that appear in the user quality requirements (Partial network, Algorithm 14).

c) All the criteria defined in the initial ANP network of a domain, regardless of those appearing in the user quality requirements (Complete network, Algorithm $15)$.

The second aspect of the model extraction methods is a set of criteria according to which the alternatives are compared to, and there are two possible cases; alternatives can be compared with respect to:

a) Only those criteria that appear in the user quality requirements (Requirements comparison).

b) All the criteria in the model (i.e., the AHP hierarchy or the ANP network) of the user's problem (Complete comparison). 


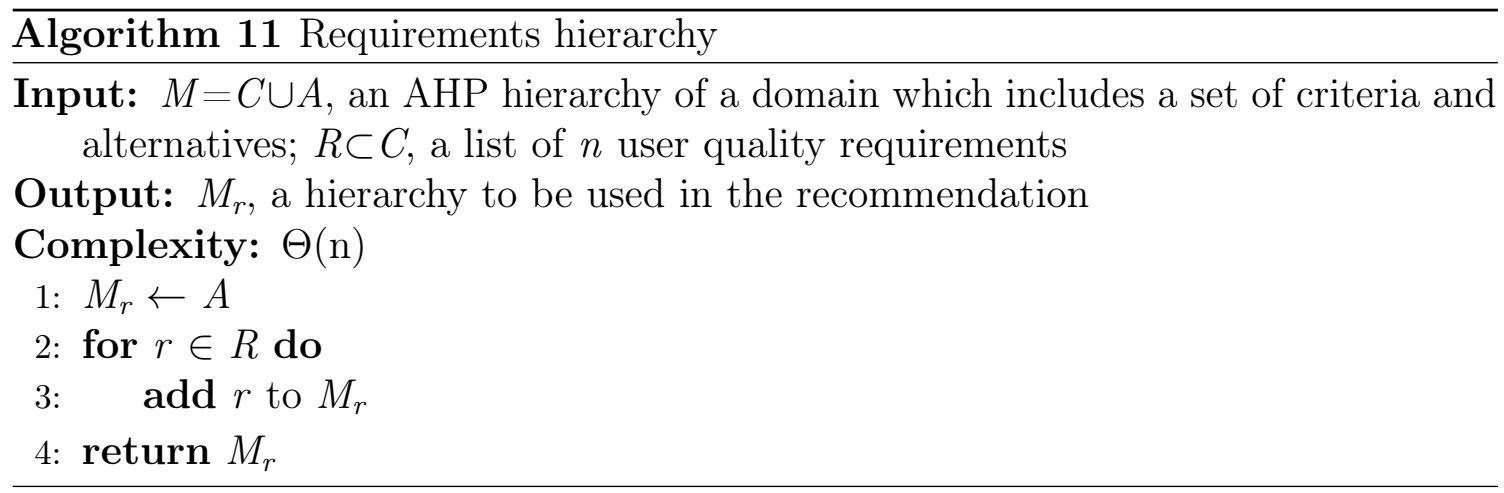

Algorithm 12 Complete hierarchy

Input: $M=C \cup A$, an AHP hierarchy of a domain which includes a set of criteria and alternatives

Output: $M_{r}$, a hierarchy to be used in the recommendation

Complexity: $\Theta(1)$

1: $M_{r} \leftarrow M$

2: return $M_{r}$

For any specific domain, an initial model is defined manually by experts only once starting from a quality model as described in Section 4.1, and it includes all the criteria relevant for that domain. Based on the user quality requirements, a sub-model can be extracted automatically using one of the extraction methods and, therefore, experts are not needed for these tasks. This minimizes the effort needed from experts, thus making the recommendation process less expensive.

Illustrative example. For the purpose of this example, let us assume that a user needs to perform a task which consist in modifying existing ontologies and matching their concepts to other ontologies; that initial ANP model of the domain created by experts is defined on Figure 4.14. For this task, let us assume that there are four

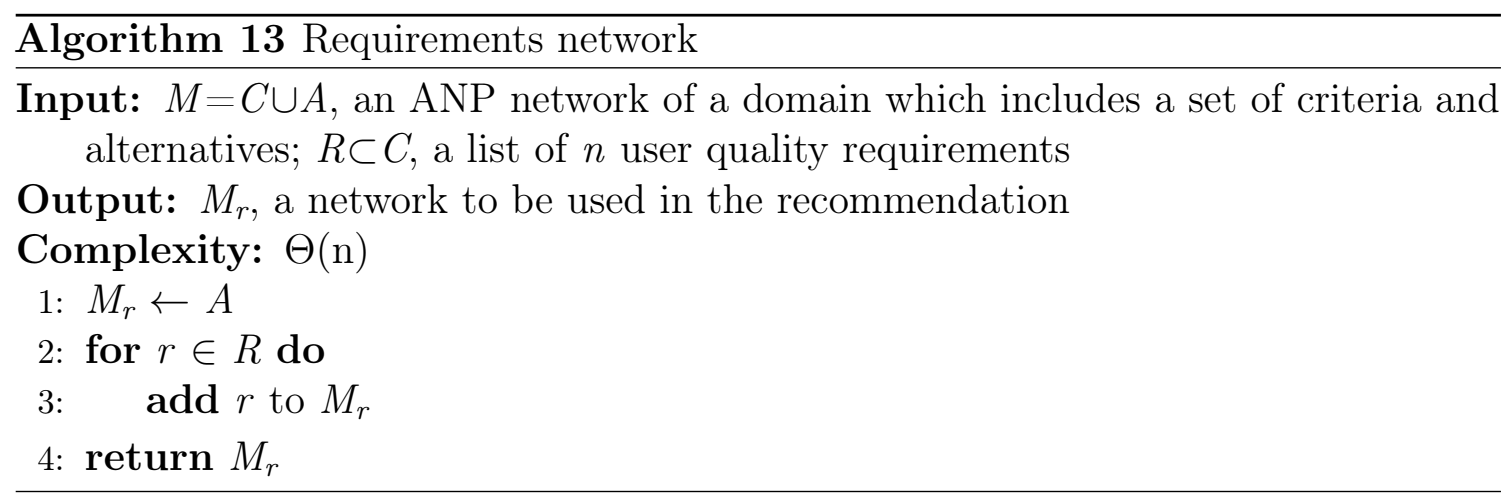


Chapter 4. The RIDER Recommendation Framework
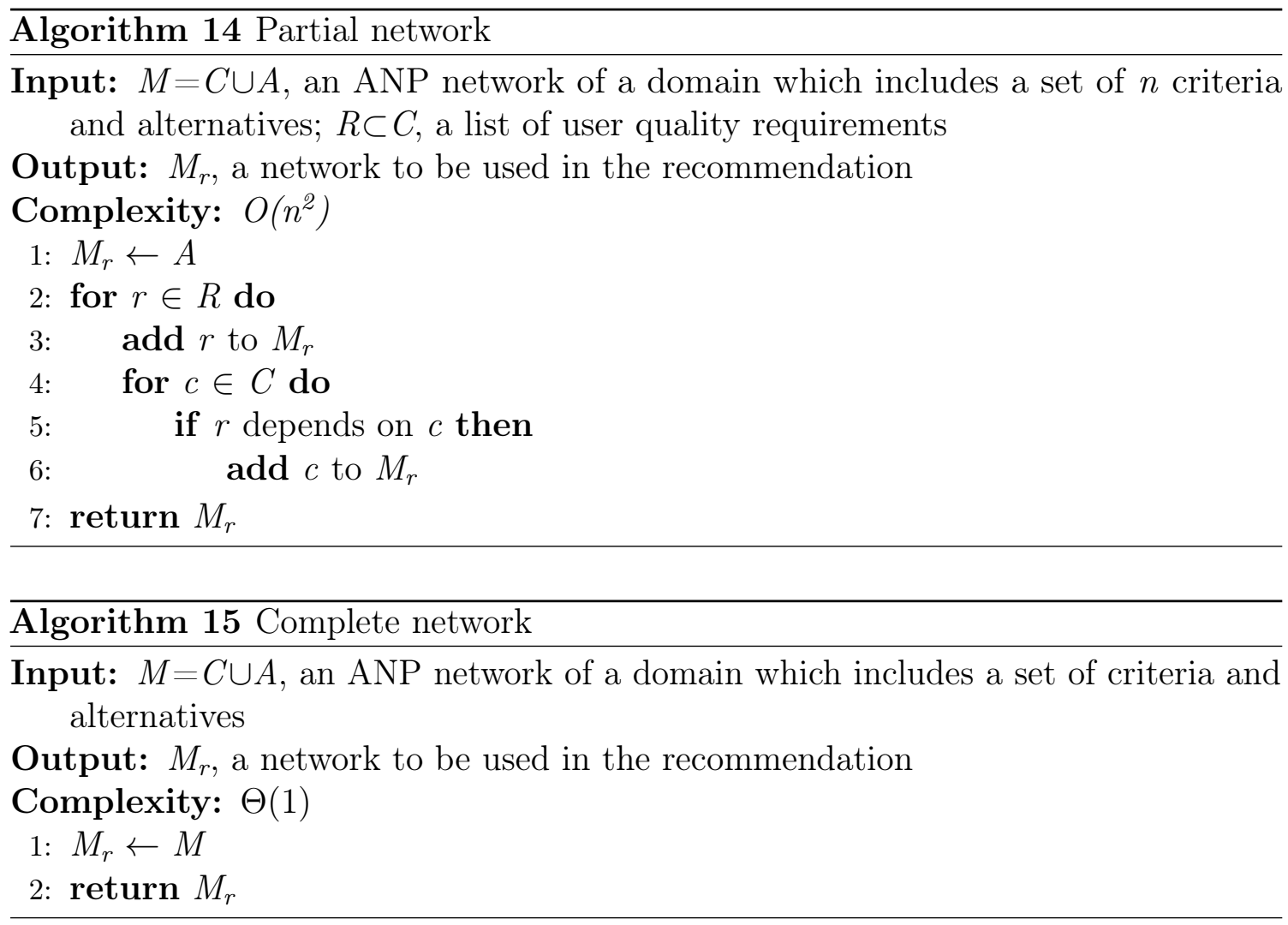

available alternatives $\left(A_{1}-A_{4}\right)$.

Let us also assume that concrete quality requirements by the user are as follows: he or she wants a tool that covers at least $80 \%$ of the ontology language components, has less than $3 \%$ errors when importing and exporting ontologies, and has at least 0.75 F-measure.

Based on these user quality requirements and on the available alternatives, the ANP network for this specific problem can be created based on the initial network (Figure 4.14), using one of the network extraction possibilities. Regarding the criteria that appear in the network, the extraction method of the ANP network chosen for this example is the one that produces a network that consists of user quality requirements and the criteria that influence them (Partial network, method $b)$. Regarding the comparison of alternatives, the chosen option is the comparison of alternatives only according to criteria that appear in user quality requirements (Requirements comparison, option $b$ ). The network produced in this example is presented in Figure $4.15^{8}$.

\footnotetext{
${ }^{8}$ For the sake of simplicity, the arcs representing dependencies between the clusters are omitted.
} 


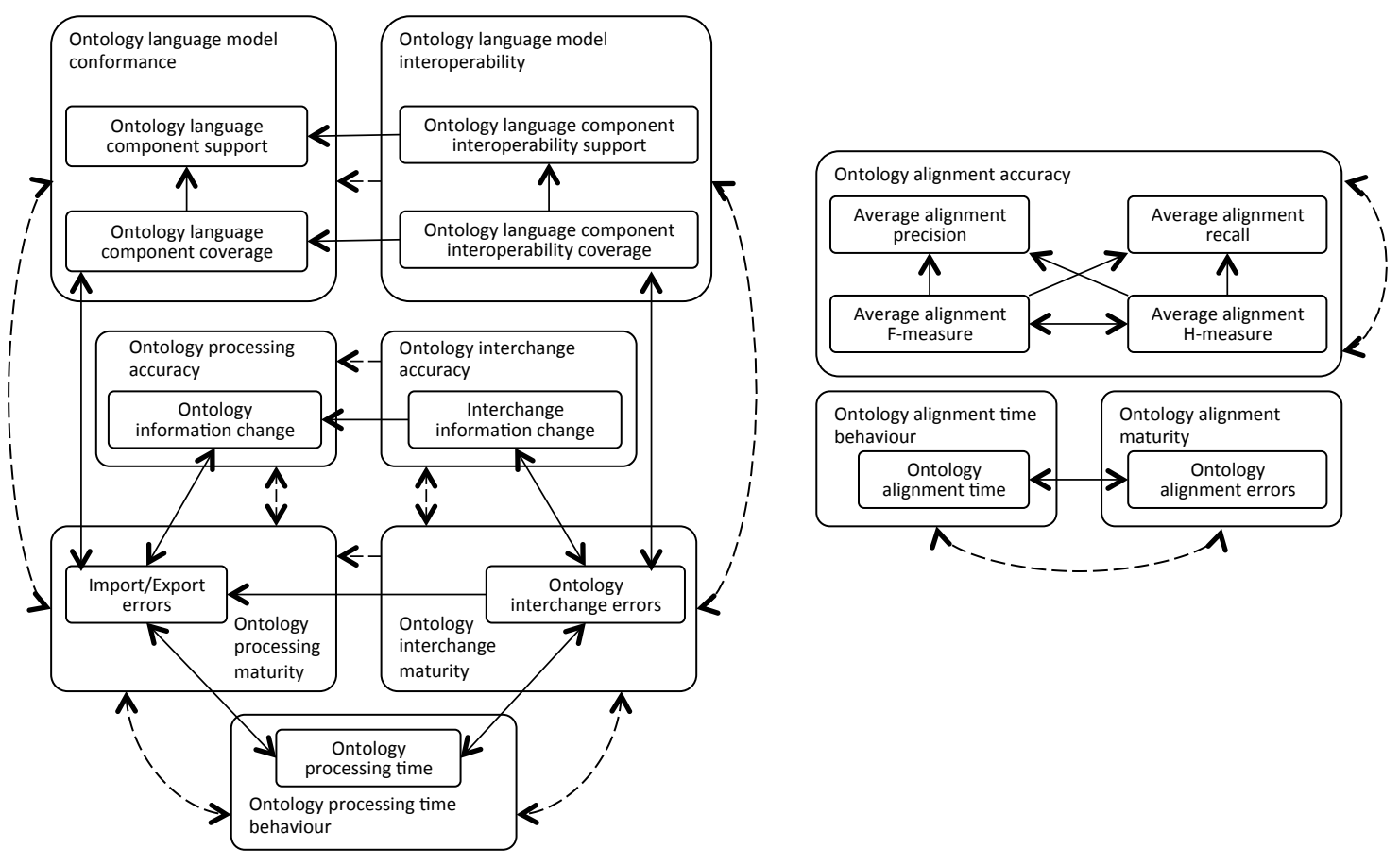

Figure 4.14: An example of the ANP network of a domain.

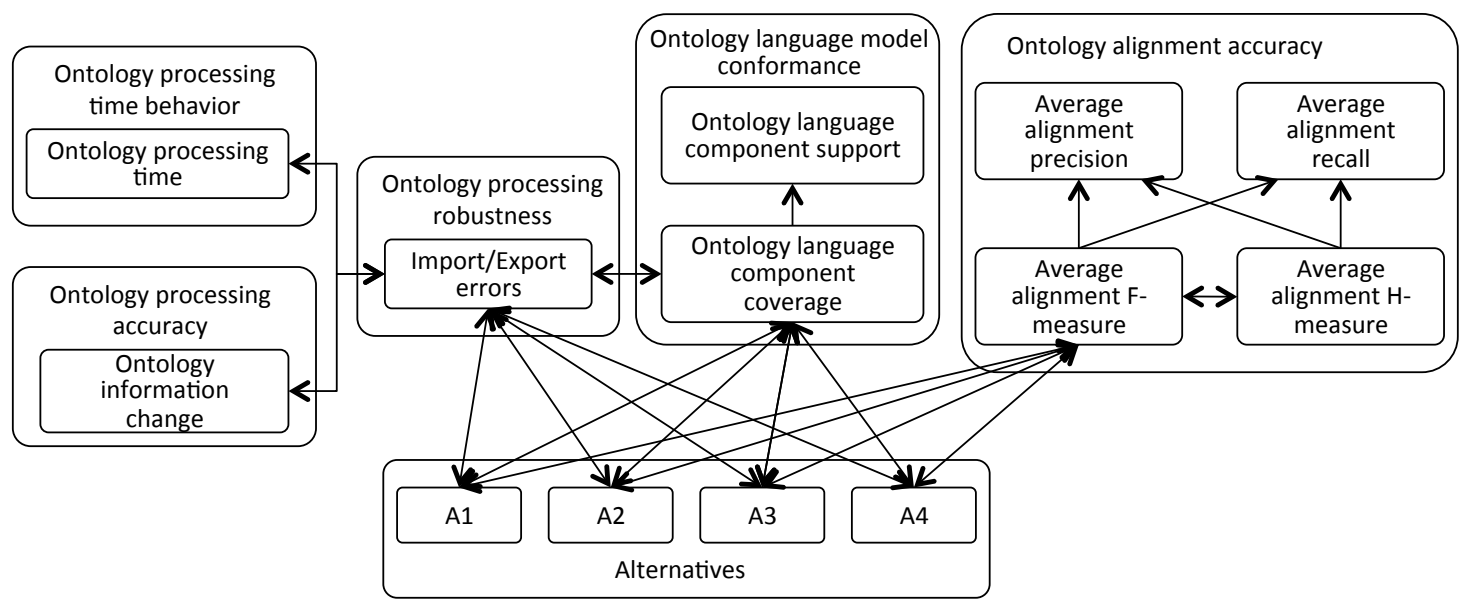

Figure 4.15: The ANP network after the network extraction process. 


\subsection{Criteria Pairwise Comparisons}

The AHP requires pairwise comparisons of all the criteria in order to determine their importance, i.e., weights (Section 2.1.4). In this method, pairwise comparisons are performed by users or by experts. Similarly, in the ANP, after a network is defined a supermatrix is constructed and its entries are filled with influence priorities calculated through pairwise comparisons of criteria and alternatives (as described in Section 2.1.5) that are performed by users or experts as well.

In RIDER, pairwise comparisons for the criteria and clusters (Figure 4.16) in order to determine their importance in both the AHP and the ANP are performed by experts in the field (this is a requirement of the RIDER framework). In the AHP, these pairwise comparisons are used to determine the importance of the criteria, which are then used directly for producing recommendations. In the ANP, these pairwise comparisons are used for the definition of the ANP supermatrix that, in RIDER, is defined in the following way:

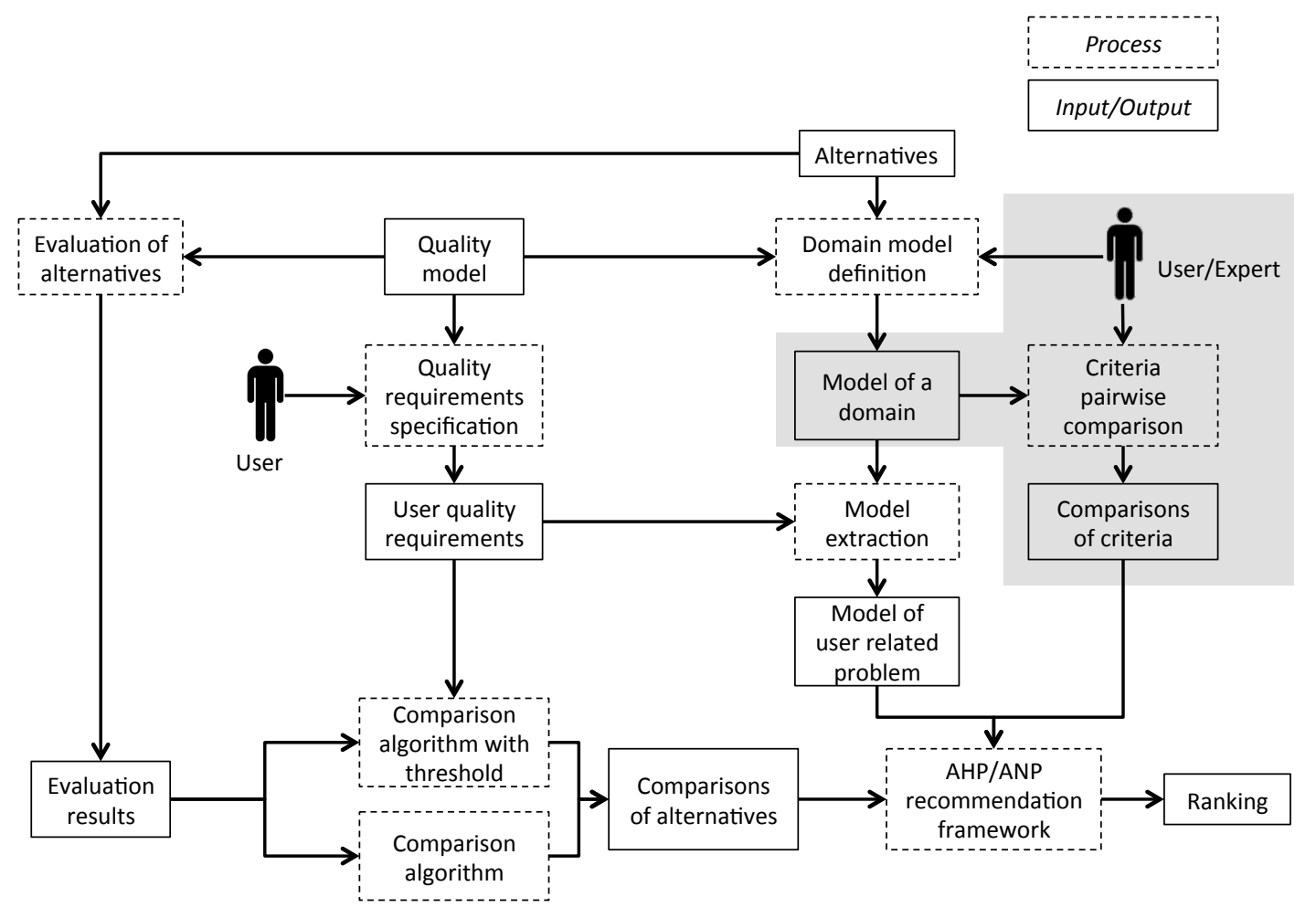

Figure 4.16: RIDER framework with criteria pairwise comparison component.

1. A set of user quality requirements is specified by a user (as described in Section 4.4). 
2. After the ANP network is obtained following a network extraction process (Section 4.5), a supermatrix that is related to the user problem is defined.

3. Entries related to criteria are filled based on the pairwise comparisons performed by experts.

4. Entries related to alternatives are filled based on the pairwise comparisons obtained by the comparison algorithm (Section 4.3 and Section 4.4), which exploits evaluation results of alternatives.

Therefore, based on a model that is defined for any specific domain, RIDER requires all the criteria pairwise comparisons to be performed by experts according to the rules defined by the method to be used in this domain (the AHP or the ANP). Furthermore, the consistency of every pairwise comparison matrix should be checked using the consistency ratio (Section 2.1.4).

Illustrative example. For this illustrative example, let us consider the ANP network represented on Figure 4.14 as the initial domain network. As an example, Table 4.4 presents the pairwise comparisons with respect to Average alignment $F$ measure (AAF). Since Average alignment F-measure depends on three elements (Average alignment precision (AAP), Average alignment recall (AAR), and Average alignment $H$-measure $(A A H)$ ) that all belong to the same cluster (Ontology alignment accuracy), these three elements have to be compared in order to determine their importance with respect to Average alignment F-measure.

Table 4.4: An example of criteria pairwise comparison with respect to Average alignment F-measure ${ }^{9}$.

\begin{tabular}{|c|c|c|c|c|}
\hline $\begin{array}{c}\text { Average } \\
\text { alignment } \\
\text { F-measure }\end{array}$ & $\begin{array}{c}\text { Average } \\
\text { alignment } \\
\text { precision }\end{array}$ & $\begin{array}{c}\text { Average } \\
\text { alignment } \\
\text { recall }\end{array}$ & $\begin{array}{c}\text { Average } \\
\text { alignment } \\
\text { H-measure }\end{array}$ & Importance \\
\hline AAP & 1 & 1 & 6 & 0.462 \\
\hline AAR & 1 & 1 & 6 & 0.462 \\
\hline AAH & $1 / 6$ & $1 / 6$ & 1 & 0.076 \\
\hline
\end{tabular}

In order to show how the ANP supermatrix is formed using the pairwise comparisons and the comparison algorithms, let us consider the comparison of alternatives by using the comparison algorithm as described in the example in Section 4.4, the user quality requirements and the related ANP network described in the example in Section 4.5, as well as the pairwise comparison example presented in this section.

\footnotetext{
${ }^{9} \mathrm{AAP}$ - Average alignment precision, AAR - Average alignment recall, AAH - Average alignment H-measure.
} 
The supermatrix corresponding to the example ANP network (Figure 4.15) is shown in Table 4.5. It can be observed that the supermatrix rows and columns represent the criteria and the alternatives from the ANP network. Therefore, the supermatrix can be divided into four parts (which in the figure are divided by double lines).

The first part of the supermatrix consists of the entries that correspond to those columns and rows that are related to the criteria in the ANP network; these entries are filled with the pairwise comparisons of criteria. For example, Ontology processing time depends only on Import/Export errors and, therefore, the entry that corresponds to the Ontology processing time column and the Import/Export errors (IEE) row is filled with the value of 1. Similarly, since Import/Export errors depends on three elements (Ontology language component coverage, Ontology processing time, and Ontology interchange errors) which all belong to different clusters, the value of 1 is inserted in each corresponding entry in the Import/Export errors column. In those cases when one element in the ANP network depends on more than one other element from the same cluster, the corresponding entries of the supermatrix are filled with the results of pairwise comparisons. For example, since Average alignment F-measure depends on three elements from the same cluster, the pairwise comparison with respect to Average alignment F-measure (Table 4.4) is used to fill the corresponding entries in the Average alignment F-measure column. In an analogous way, the entries in the Average alignment $H$-measure column are filled with the pairwise comparison of criteria with respect to Average alignment H-measure.

The second part of the supermatrix consists of the entries that correspond to those columns that are related to criteria, and to those rows that are related to alternatives; these entries are filled with the pairwise comparisons of alternatives with respect to criteria by using the comparison algorithms and by exploiting evaluation results. For example, the pairwise comparison of alternatives with respect to Ontology language component coverage (Table 4.3) is used to fill the corresponding entries in the supermatrix related to the Ontology language component coverage column. Similarly, the pairwise comparisons of alternatives with respect to Import/Export errors and to Average alignment F-measure are used to fill the corresponding entries related to the Import/Export errors and to the Average alignment F-measure columns, respectively.

The third part of the supermatrix consists of the entries that correspond to those columns that are related to alternatives, and to those rows that are related to criteria; these entries are filled with the pairwise comparison of criteria with respect to alternatives. For example, since $A 1$ depends on three elements which all belong to different clusters, the value of 1 is inserted in each corresponding entry in the $A 1$ column. Similarly, the entries that correspond to the A2, A3, and $A_{4}$ columns are filled. 


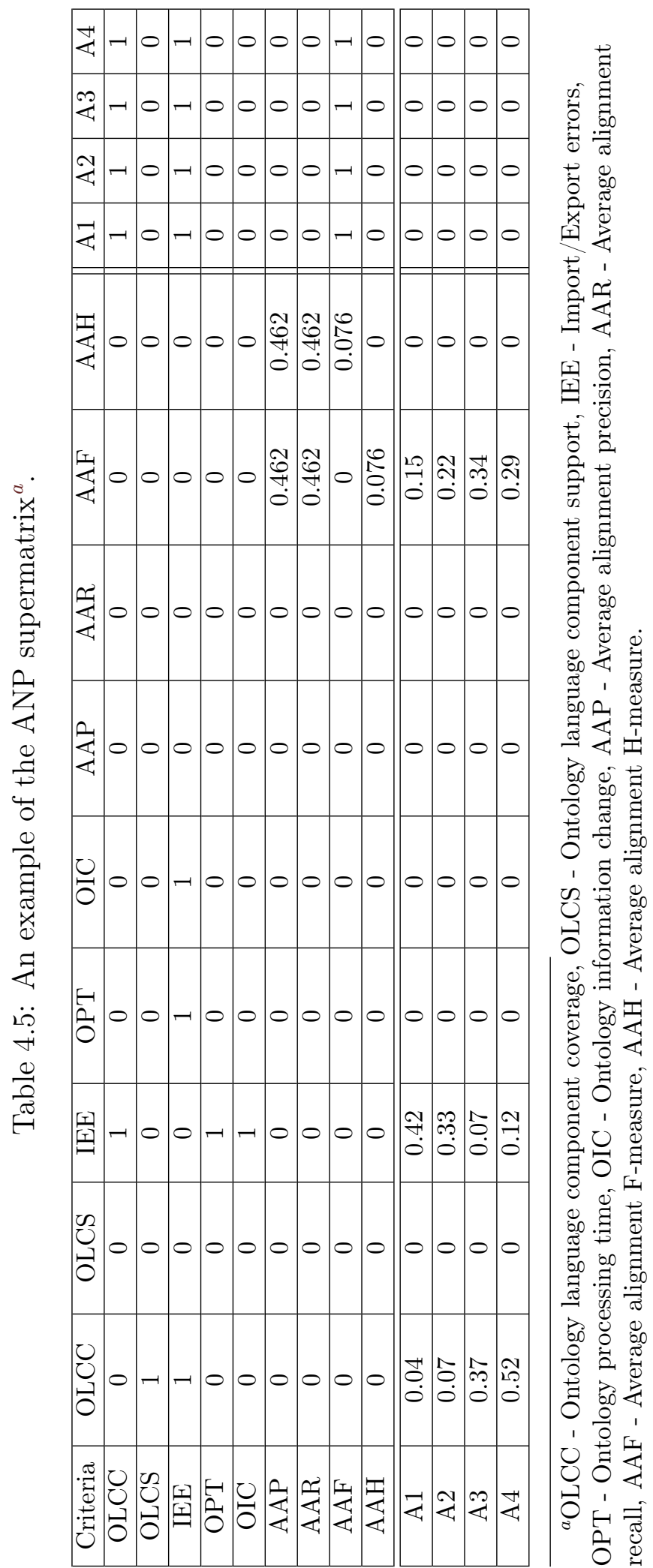


The fourth part of the supermatrix consists of the entries that correspond to those columns and rows that are related to alternatives; these entries are filled zeros, since alternatives do not depend on each other.

\subsection{Instantiation of the RIDER Recommendation Framework}

RIDER is a generic recommendation framework that can be instantiated in any domain and that is useful when evaluation results of different alternatives are available. The framework is instantiated only once for a particular domain; afterwards, it provides recommendations to users based on their quality requirements.

In order to successfully instantiate RIDER, there are several artefacts that must be provided as inputs by the experts in a particular domain, which are specified only once for each domain:

- Quality model in a domain of application. As described in Section 4.1 quality models provide a framework for the evaluation of alternatives and a basis for the creation of the AHP and the ANP model of a domain.

- The model of the domain of application. The model of a domain is the starting point in recommendations using the AHP and the ANP. When instantiating the RIDER framework in a particular domain, an initial model of the complete domain is required (Section 4.5); such model can be obtained using the quality model, as described in Section 4.1. This AHP or ANP model is defined by experts only once for a particular domain.

- Criteria pairwise comparisons related to the criteria in the model of the domain. These comparisons are important because they are used for determining the weights of the criteria and for constructing the ANP supermatrix (Section 4.6). These pairwise comparisons are performed by experts.

- Evaluation results of the set of alternatives in which the recommendations will be based. Evaluation results are important for the RIDER framework because the comparison of alternatives according to criteria is performed automatically, based on these evaluation results, by using comparison algorithms (Section 4.3 and Section 4.4). Furthermore, in the instantiated RIDER evaluation results can be updated as new results become available, or added incrementally to the instantiation.

When using the instantiated RIDER framework, the role of users or experts is to a certain extent reduced compared to the ANP/AHP (Figure 4.17), and a typical use case in a certain domain consists of the following: 
- A user who seeks a recommendation specifies quality requirements, i.e., those characteristics that are important for him or her. These quality requirements are inputs in a recommendation process, and no other input is required from users.

- Based on user quality requirements, the model of the user-related problem is automatically constructed through the model extraction process.

- Alternatives are compared automatically using comparison algorithms, based on user's quality requirements and evaluation results of alternatives.

- Criteria are compared automatically, based on the initial criteria pairwise comparisons provided by the experts upon the RIDER instantiation.

- A ranking of alternatives is obtained automatically and presented to users as output.

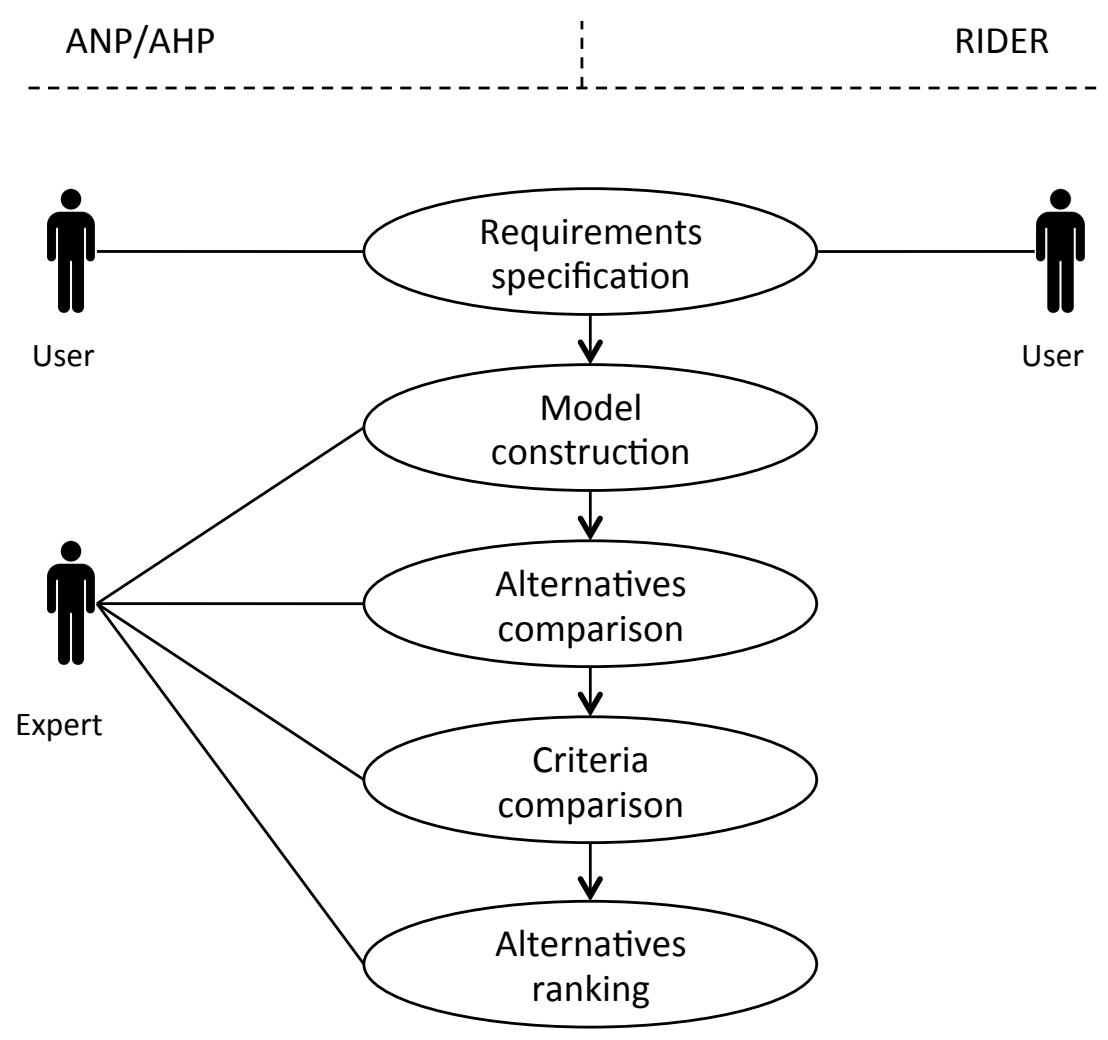

Figure 4.17: ANP/AHP and RIDER use case diagram. 


\subsection{RIDER Software Support}

The RIDER recommendation framework provides a flexible methodology for recommendation based on evaluation results and user quality requirements. The main value of the framework is that it is domain-independent and modular, and that it provides the automatic adaptation of a decision process to any user in a particular domain.

RIDER has been implemented as a Java library, which allows developers to use the framework in their applications. Furthermore, a generic web application for recommendation has been developed, which can be instantiated in any domain and provide users with recommendations based on their quality requirements.

\subsubsection{The jRIDER Java Library}

The jRIDER library is a Java library that implements methods and functionalities of the RIDER framework, which include AHP and ANP-related methods and RIDER extensions.

All the versions of the comparison algorithms without user quality requirements (Section 4.3) and with user quality requirements (Section 4.4) are implemented in the jRIDER library. The four types of scales are supported in both cases, allowing developers to automatically perform comparisons using the algorithms.

The jRIDER library also includes classes designed to utilize evaluation results that are described according to the Evaluation Result ontology (Section 4.2.1), as well as information about quality models described according to the Quality Model ontology (Section 4.1.1). Evaluation results that are described according to this ontology can be loaded into the jRIDER library and be automatically processed. Also, this set of classes provides methods for performing SPARQL queries over evaluation results.

The jRIDER library provides functionalities relevant to the AHP, the ANP, and RIDER related to:

- Performing basic operations over a matrix (normalizing columns and determining whether the matrix is stochastic or convergent, checking the consistency of a matrix (Section 2.1.4)).

- Performing operations related to comparisons (pairwise comparisons of criteria for calculating weights, pairwise comparisons of alternatives using the comparison algorithms (Section 4.3 and Section 4.4)).

- Performing operations related to the supermatrix (instantiating a model according to the described methods (Section 4.5) and constructing the related supermatrix, filling the supermatrix with pairwise comparisons, calculating the limit supermatrix). 
Furthermore, the jRIDER library provides some advanced functionalities which include:

- Automatically constructing a supermatrix based on a dependence matrix, user quality requirements, and a set of criteria pairwise comparisons.

- Automatically comparing alternatives in a given supermatrix according to evaluation results.

- Obtaining recommendations based on the weighted supermatrix.

\section{Architecture}

The jRIDER library is divided into several packages, which correspond to the components in the RIDER framework (Figure 4.18). Therefore, separate packages provide classes for:

- Processing and querying evaluation results stored in the RDF format.

- Comparing the alternatives using the comparison algorithms.

- Instantiating the model according to the model extraction methods.

- Performing the operations related to the ANP and the AHP methods.

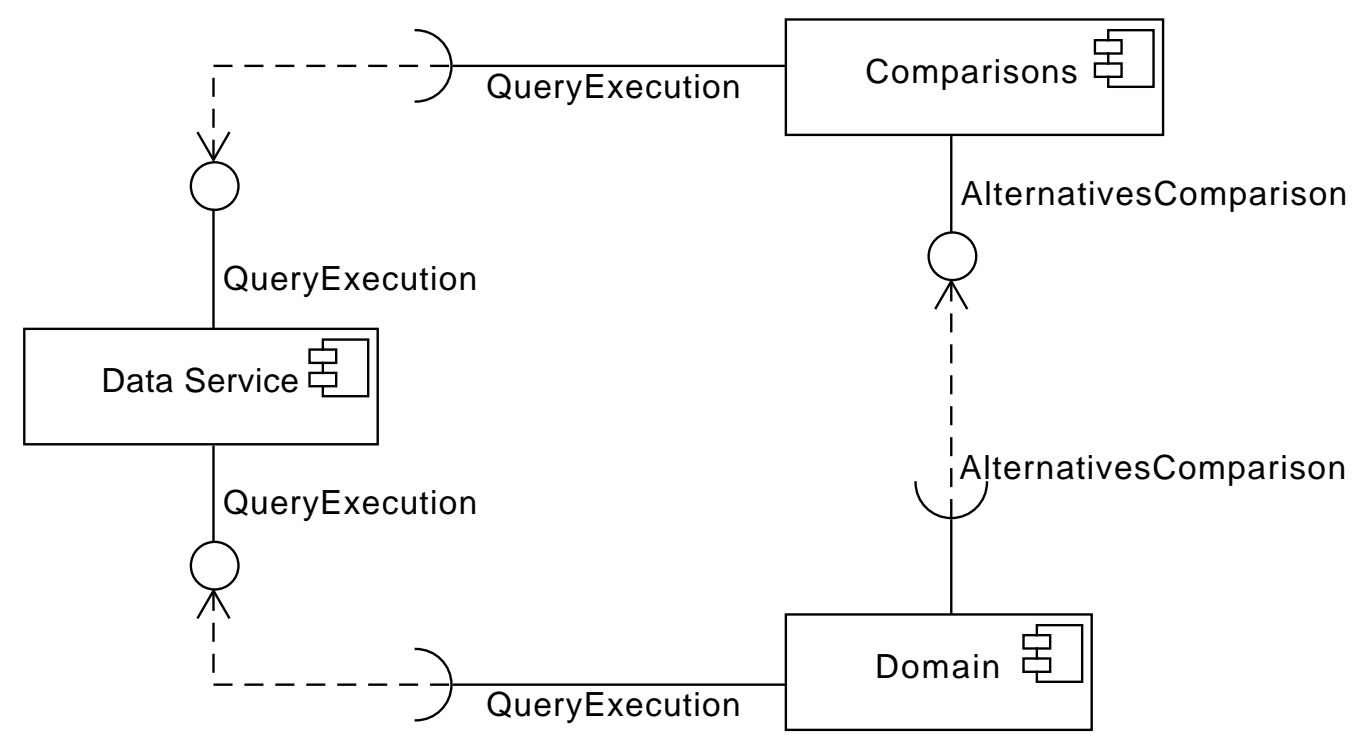

Figure 4.18: jRIDER architecture. 


\section{Dependencies}

In the implementation of the jRIDER library, Maven ${ }^{10}$ is used for project and dependencies management. It has become practically a standard in the software development process and provides easy maintenance and reusability. Maven is licensed under the Apache 2.0 license $^{11}$.

The JAMA ${ }^{12}$ library is used in the development of the library. It provides some functionalities and operations for matrices, which are extended in the jRIDER library. JAMA library has been released to the public domain and can be freely used.

JenaBean $^{13}$ is an annotation-based framework built on top of the Jena ${ }^{14} \mathrm{RD}$ F/OWL API. Using JenaBean, an object-oriented Java model is bound to the ontology that is used for representing evaluation results. That way, RDF data can be easily managed using Java objects. JenaBean and Jena are licensed under the Apache 2.0 license.

\subsubsection{The RIDER Web Application}

The RIDER web application is a generic web application for recommendation that can be instantiated in any domain. It is implemented in Java as a web application, using some well-known open-source frameworks and libraries.

\section{Architecture}

The architecture of the RIDER web application describes several separate components (Figure 4.19):

1. Quality model - quality indicators that are described in the quality model (Section 2.2.5) help in obtaining evaluation results and are used to describe the criteria in the recommendation process. Furthermore, quality indicators are a reference for the description of user quality requirements.

2. Evaluation Result ontology and Quality Model ontology - the ontologies for representing data about evaluation results and quality models. Such ontologies capture data about quality indicators (i.e., criteria in the recommendation process), their evaluation results and alternatives. The Quality Model ontology and the Evaluation Result ontology are described in detail in Sections 4.1.1 and 4.2 .1 , respectively.

\footnotetext{
${ }^{10}$ http ://maven . apache.org/

${ }^{11}$ http : //apache .org/licenses/LICENSE - 2.0.txt

12 http://math.nist.gov/javanumerics/jama/

${ }^{13}$ http://code.google.com/p/jenabean/

${ }^{14}$ http://jena. sourceforge. net/
} 


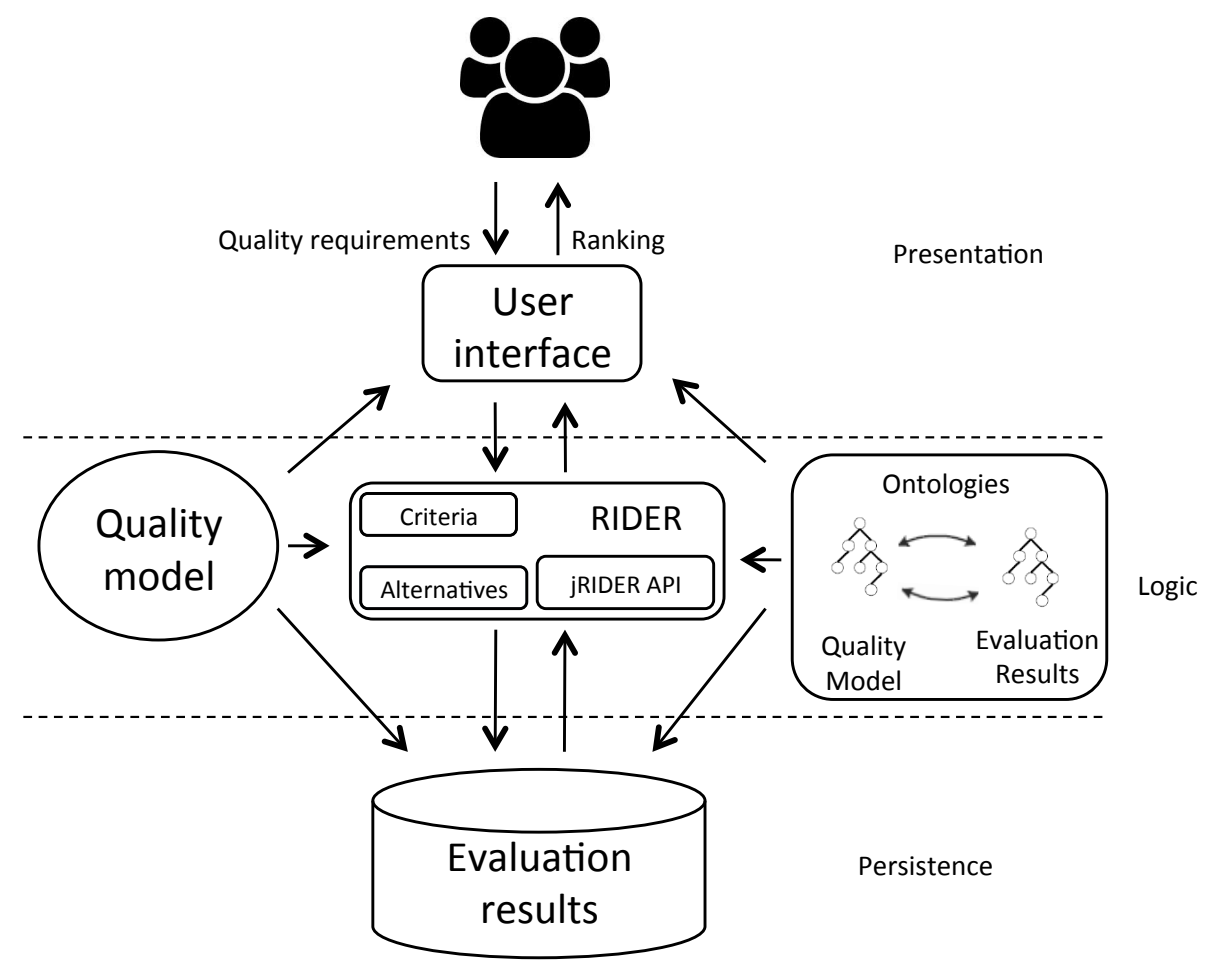

Figure 4.19: Architecture of the RIDER web application.

3. Evaluation results - results of the evaluation of alternatives with respect to quality indicators (criteria) which are obtained using the quality model, and which are described using the Evaluation Result ontology and the Quality Model ontology. These results are used by the RIDER application logic in the comparison of alternatives in the recommendation process, and are stored in the RDF files.

4. RIDER application logic - the RIDER application logic is the key part of the system and is in charge of processing user quality requirements and evaluation results, and of ranking the possible alternatives. It implements the RIDER framework, and uses the jRIDER library described in the previous section.

5. User Interface (UI) - the user interface is the bridge between the user and the system. The user interacts with the system through the UI by specifying his/her quality requirements, which are forwarded to the RIDER application logic. When the recommendation process is finished, the results are presented to the user through the UI. 


\section{Dependencies}

In the implementation of the recommendation system for semantic technologies, similarly as in the case of the jRIDER library, Maven is used for project and dependencies management.

Tapestry ${ }^{15}$ is an open-source framework for building dynamic and robust web applications in Java. It complements and builds upon the standard Java Servlet API and therefore it works in any servlet container or application server. Developing Tapestry applications involves creating HTML templates using plain HTML and combining the templates with small amounts of Java code. In Tapestry, applications are created in terms of objects and the methods and properties of those objects and specifically not in terms of URLs and query parameters. Tapestry is licensed under the Apache 2.0 license.

Spring ${ }^{16}$ is an open-source application framework for the Java platform, and it is used as an Inversion of Control container. The container is responsible for managing object lifecycles, and objects are obtained by means of Dependency Injection where the container provides objects by name and which allows for high flexibility in the implementation. Spring is licensed under the Apache 2.0 license.

\section{Instantiation}

The RIDER web application is generic and can be instantiated in any domain taking into account several requirements. These requirements follow from the general artefacts for RIDER instantiation (see Section 4.7), and include:

- Evaluation results (values for quality indicators obtained in the evaluation, as well as alternatives to be recommended) have to be described according to the Evaluation Result ontology (Section 4.2.1). Furthermore, evaluated quality indicators and related quality model have to be described according to the Quality Model ontology (Section 4.1.1). Data stored in RDF format and according to previously mentioned ontologies can be used in the web application without any code modification.

- A set of pairwise comparisons related to quality indicators, and another set related to quality characteristics has to be described using JSON ${ }^{17}$. These pairwise comparisons have to be performed by experts, and in accordance to the previously specified model (AHP hierarchy or ANP network). Furthermore, in the case that an ANP is used, dependencies between all the indicators have to be described in another JSON file.

\footnotetext{
${ }^{15}$ http: //tapestry.apache.org/

${ }^{16}$ http://Www. springsource.org/

${ }^{17}$ http://www.json.org/
} 
- A configuration file has to be specified, which defines the method to be used in the recommendation (ANP or AHP), as well as the specific comparison algorithm (Sections 4.3 and 4.4) and model extraction method (Section 4.5).

\subsection{Summary}

This chapter has presented RIDER, a recommendation framework built on top of the AHP and the ANP, which is specifically designed to take into account evaluation results of alternatives and user quality requirements. To this extent, this chapter has presented the work carried out in this thesis in order to achieve the first objective, to provide a Multiple Criteria Decision Making framework that extends the Analytic Network Process and that takes advantage of evaluation results of alternatives and of user quality requirements over such alternatives.

In the context of achieving the first objective, this chapter has presented the first contribution of this thesis, C1: domain-independent algorithms for the automatic comparison of alternatives according to alternatives' evaluation results (Sections 4.3 and 4.4). These algorithms take evaluation results and user quality requirements as inputs, and provide their outputs in Saaty's pairwise comparison scale.

This chapter has also presented the second contribution of this thesis, C2: a set of methods for the dynamic extraction of the AHP and ANP models that are based on user quality requirements (Section 4.5). These methods take a set of user quality requirements and an initial AHP or ANP model in a domain of application as inputs, and provide outputs in terms of the AHP or the ANP model specifically adapted to the input set of user quality requirements.

RIDER consists of several components that can be exploited independently one from another, each described in detail in this chapter, and is a generic recommendation framework that can be instantiated in any domain. RIDER enables the automation of a recommendation process, requiring minimal inputs from experts or users in order to deliver recommendations in any domain of application, and can be considered as the backbone of this thesis, providing the foundations for achieving the thesis second objective presented in the following chapters.

Finally, this chapter has also presented the third contribution of this thesis, C3: software that supports the proposed MCDM framework. RIDER software helps in putting RIDER into practice and in delivering recommendations to users in the domain of instantiation. 



\section{Chapter 5}

\section{SemQuaRE - A Quality Model for Semantic Technologies}

RIDER is a general recommendation framework that can be applied to any domain provided that it is correctly instantiated (Section 4.7). The next two chapters in this thesis describe the instantiation of the RIDER framework in the semantic technology domain.

Since quality models are significant for the evaluation of alternatives and are a basis for the creation of the AHP or the ANP model of a domain, one of the artefacts that is needed for the instantiation of RIDER is a quality model in the domain of application. Although a quality model is an important part of the RIDER framework, it is also a valuable resource independent of RIDER (Section 2.2); therefore, this chapter describes SemQuaRE, a software quality model in the domain of semantic technologies.

SemQuaRE is a hierarchical quality model which extends the ISO SQuaRE one. It describes base measures, derived measures and quality indicators at the lower level, and quality sub-characteristics and quality characteristics adopted from SQuaRE at the higher level (Figure 5.1). SemQuaRE describes six different types of semantic technologies: ontology engineering tools (Section B.1), reasoning systems (Section B.2), ontology matching tools (Section B.3), semantic search tools (Section B.4), and semantic web service tools (Section B.5) have been defined using evaluation results from the SEALS project (Section 2.3.2), while ontology annotation tools (Section B.6) have been added in the process of evaluation (Section 7.4.2).

As discussed in Section 2.5, existing software quality models provide terminology and descriptions of characteristics that are general. Therefore, in order to apply them in a particular domain, these quality models usually have to be extended by applying one method for extending quality models (Section 2.2.6). The existing methods for extending quality models follow the top-down approach and, although in some cases it 


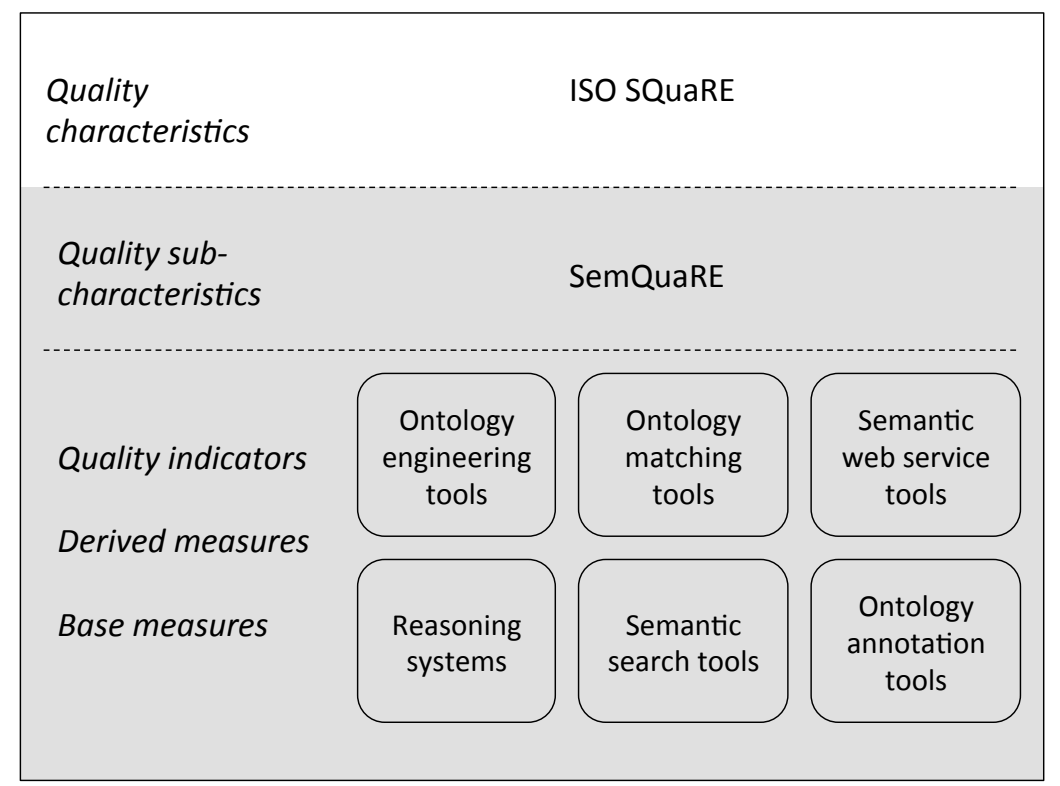

Figure 5.1: Structure of the quality model for semantic technologies.

can be useful to base the definition of a quality model on already available evaluation results, no bottom-up approach has been developed to this date. Therefore, this chapter first presents a bottom-up method for extending software quality models and, afterwards, a detailed description of how this method has been applied for the construction of the quality model for semantic technologies.

\subsection{Bottom-up Method for Extending Software Qual- ity Models}

As discussed in Section 2.5, all methods for extending software quality models follow the top-down approach and, although in some scenarios a bottom-up approach would be more effective to apply, no bottom-up approach for extending software quality models has been described in the literature.

This section introduces a method for extending software quality models which is based on a bottom-up approach. In this method, test data and evaluation results are used as the starting point from which the measures, sub-characteristics and characteristics are specified.

The bottom-up method for extending a software quality model consists in performing six consecutive steps (Figure 5.2). Next, each step is described, including examples for the case of ontology matching tool evaluation: 


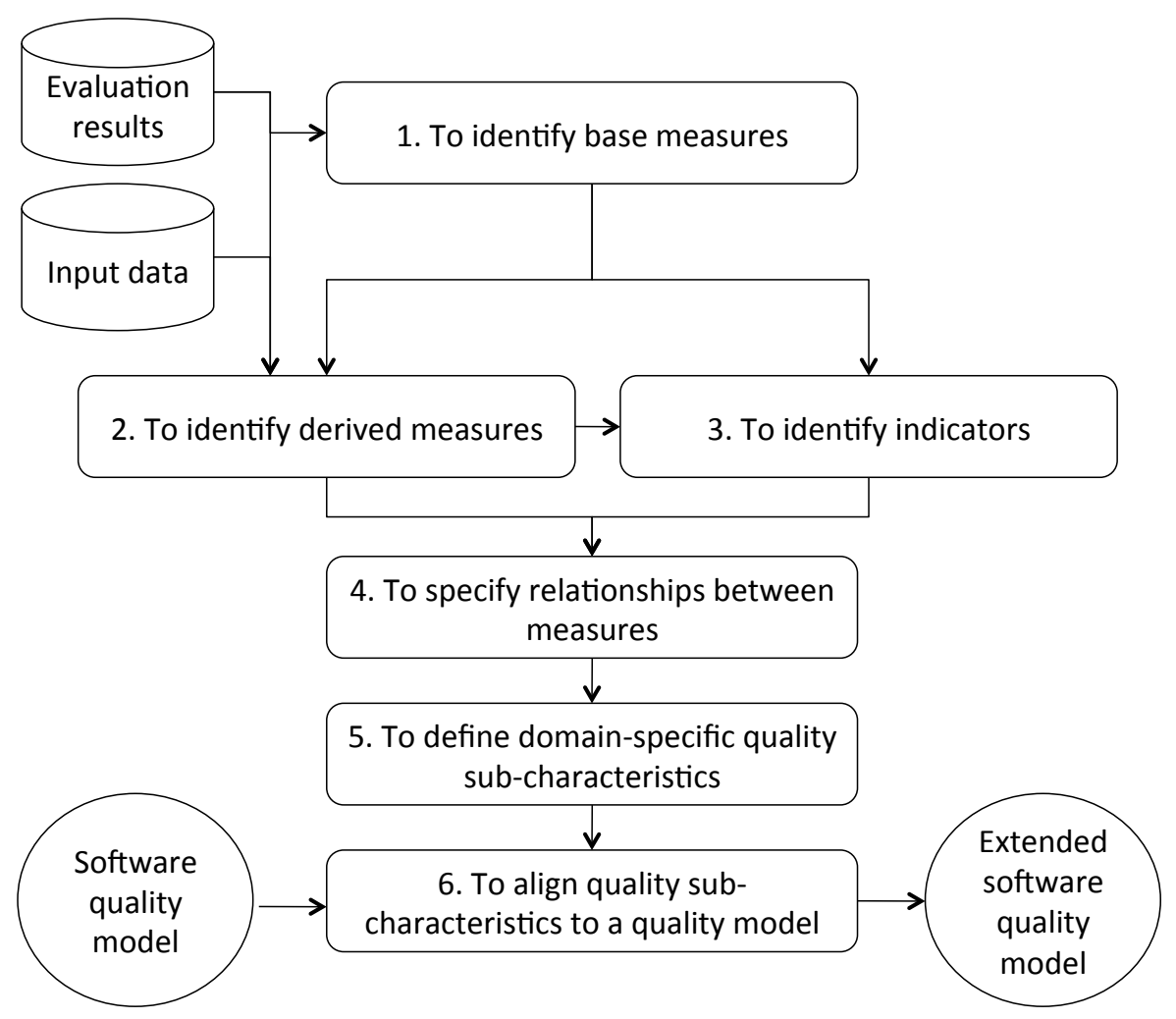

Figure 5.2: The method for extending software quality models based on a bottom-up approach.

1. To identify base measures. The output of evaluating a software product in a single test with some input data allows identifying the base measures.

For example, in ontology matching tool evaluation, base measures could be output alignment, representing the alignment produced by the tool in a task, or memory consumption in a task.

2. To identify derived measures. Base measures can be combined among them and/or with input data in order to obtain derived measures. Derived measures are defined in a way that they bring additional and meaningful information not provided by the base measures themselves; it is also possible to use one base measure in order to obtain more than one derived measure. In some cases it is possible to combine different derived measures to obtain other derived measures.

Derived measures are usually related to one test. However, in some cases a derived measure can be obtained by combining base measures from a number 
of tests.

A derived measure for ontology matching tools could be alignment precision.

3. To identify indicators. Indicators are measures related to a whole evaluation (i.e., a number of tests) and are obtained by the aggregation of base measures and/or derived measures. As in the previous case, a base or derived measure can be used in more than one indicator. For each indicator, a scale and a unit of measurement should be specified.

In some cases, it is possible to perform several evaluations using different types of input data (e.g., different ontology languages for each evaluation). In these cases, values for indicators can be obtained as a combination (e.g., by averaging) of values produced in each evaluation.

In the case of ontology matching tools, an indicator could be the average alignment precision, which belongs to a ratio scale with 0 as minimum and 1 as maximum values, or average memory consumption, which belongs to a ratio scale with 0 as minimum and no predefined maximum value.

4. To specify relationships between measures. In this step, which can be performed in parallel with the previous ones, relationships between measures are expressed either in an informal way (e.g., the collaboration, damage and dependency categories proposed by Franch and Carvallo [36]) or more formally (e.g., with the formulas used for obtaining the measures, as proposed by Bombardieri and Fontana [12]). For every derived measure defined, it is recommendable to specify the formula (or set of formulas) that allows obtaining such derived measure from the base measures. Similarly, it is also advisable for any indicator to identify the formula that defines such an indicator based on other measures. Also, it is important to note that one indicator can be obtained by means of different formulas and that, in order to have consistency in the quality model, all indicators have to be obtained by means of derived or base measures that are already defined in the model.

In the ontology matching tools example, the formula for average alignment precision and average memory consumption could be the average of alignment precisions and memory consumptions measured in the evaluation, respectively.

5. To define domain-specific quality sub-characteristics. Every software product from a particular domain has some sub-characteristics that are different from other software products; those sub-characteristics, together with more generic ones, should be identified and precisely defined. Every indicator provides some information about one or several software sub-characteristics; therefore, based on the indicators defined in the previous step, software quality sub-characteristics 
are specified. It is not necessary that every quality sub-characteristic has only one indicator but rather a set of indicators that determines it. Thus, quality sub-characteristics can be examined through several different indicators which can be combined to measure certain sub-characteristics. Finally, if needed, some quality sub-characteristics can be combined into more general ones.

In order to have consistency in the quality model, all indicators should be used for quality sub-characteristics, and no quality sub-characteristic should be measured with indicators different to those already specified, nor should they be measured without any of the indicators assigned.

A quality sub-characteristic of an ontology matching tool measured using average alignment precision could be the ontology alignment accuracy, which can further be a sub-characteristic of ontology processing accuracy, and a quality subcharacteristic measured using average memory consumption could be ontology alignment resource utilization.

6. To align quality sub-characteristics to a quality model. In this step, the alignment with an existing quality model is established, i.e., the software quality subcharacteristics previously defined are related to others already specified in the existing model. Depending on the domain and nature of the software product, some new quality characteristics can be specified or existing ones can be modified or excluded.

The sub-characteristic defined for ontology matching tools in the previous step can be aligned to the SQuaRE's functional correctness and resource utilization sub-characteristic.

Next, the construction of SemQuaRE is described through each step of the presented method.

\section{$5.2 \quad$ Identifying Base Measures}

The starting point for defining software quality measures in SemQuaRE has been the set of evaluations performed in the international evaluation campaigns organized in the SEALS project (Section 2.3.2). In these campaigns, more than 40 different tools from organizations in more than 10 countries have participated producing evaluation results for different types of semantic technologies (ontology engineering tools [42, 43], reasoning systems [140, 142], ontology matching tools [88, 132], semantic search tools [137, 138], and semantic web service tools [18, 78]).

For each type of technology, different evaluation scenarios were defined, using in each of them different test data as input. In this step, the base measures of each 
evaluation scenario (i.e., those outputs directly produced by the software during the evaluation) have been identified.

In this section, only the outcomes of each step for two concrete scenarios are presented, those of evaluating the conformance of ontology engineering tools and of evaluating the accuracy of ontology matching tools (a complete description of SemQuaRE is provided in Appendix B, as well as in the SemQuaRE wiki ${ }^{1}$ ).

In the SEALS project, the evaluation process is based on the IEEE 829-2008 standard [58] and the evaluation data consists of different test suites, each containing a number of different test cases to be executed. These test suites are used for evaluating the conformance of ontology engineering tools and the accuracy of ontology matching tools.

\section{Conformance Scenario of Ontology Engineering Tools}

Conformance evaluations of ontology engineering tools assess the degree in which the knowledge representation model of a tool adheres to the knowledge representation model of an ontology language according to the different ontology components (e.g., classes, properties) of such knowledge representation models (see [38] for a complete description and use of these evaluations).

In the case of the conformance evaluation of ontology engineering tools, each of the test cases in a test suite contains:

- Origin ontology. The ontology to be used as input.

An execution of each of these test cases in a test suite consists in importing the file that contains an origin ontology $\left(O_{i}\right)$ into the tool and then exporting the imported origin ontology to another ontology file $\left(O_{i}^{I I}\right)$, as shown in Figure 5.3. The ontology $\left(O_{i}^{I}\right)$ represents the ontology inside the tool, for which the state is unknown. Therefore, the comparison of two ontologies $\left(O_{i}\right.$ and $\left.O_{i}^{I I}\right)$ gives an insight of the extent to which the tool observed conforms to the ontology model.

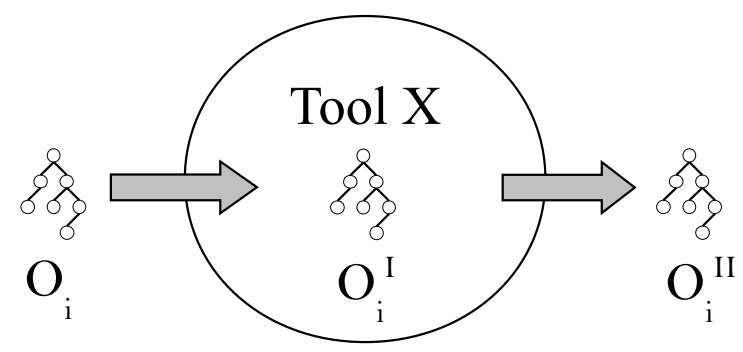

Figure 5.3: Conformance test execution of ontology engineering tools [38].

\footnotetext{
${ }^{1}$ http://semquare.oeg-upm.net
} 
The base measures obtained after a test case execution of the conformance scenario of ontology engineering tools are:

- Final ontology. The ontology produced by the tool when importing and exporting the origin ontology.

- Execution problem. Whether there were any execution problems in the tool when importing and exporting the origin ontology. Possible values are true, and false.

\section{Accuracy Scenario of Ontology Matching Tools}

Accuracy evaluations of ontology matching tools assess the degree to which a product or system provides the correct results with the needed degree of precision when performing a matching task.

In the accuracy scenario of ontology matching tools, each test case contains:

- Ontology 1. One ontology to be used as input for an alignment task.

- Ontology 2. Another ontology to be used as input for an alignment task.

- Expected alignments. The expected alignments to be obtained when aligning the two input ontologies.

A test case execution consists in importing two ontologies into the tool $\left(O_{i}\right.$ and $O_{i i}$ ), and performing the matching (alignment) task over the two ontologies (Figure 5.4). The output of the test is the alignment produced by the tool $(A)$, which is then compared to the expected alignment $(E a)$.

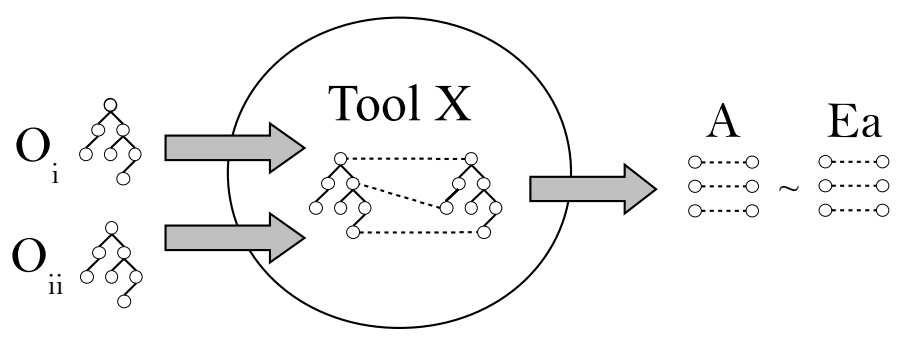

Figure 5.4: Accuracy test execution of ontology matching tools.

The base measures obtained after a test case execution of the accuracy scenario of ontology matching tools are:

- Output alignments. The alignments that are produced by the tool when aligning ontology 1 and ontology 2. 
- Alignment duration. The amount of time needed for the alignment task.

- Execution problem. Whether there were any execution problems in the tool when performing an alignment task. Possible values are true, and false.

\subsection{Identifying Derived Measures}

In this step, the base measures identified in the previous step were combined together with the test data in order to obtain derived measures.

\section{Conformance Scenario of Ontology Engineering Tools}

In the conformance scenario of ontology engineering tools, based on the test data (origin ontology) and on the base measures of one test execution (final ontology), the following derived measures were specified:

- Information added. The information added to the origin ontology after importing and exporting it.

- Information lost. The information lost from the origin ontology after importing and exporting it.

- Structurally equivalent. Whether the origin ontology and the final one are structurally equivalent.

- Semantically equivalent. Whether the origin ontology and the final one are semantically equivalent.

- Conformance. Whether the origin ontology has been imported and exported correctly with no addition or loss of information.

The derived measures previously defined give insight into additions or losses of information that are possible due to the tool's internal ontology model and to whether the semantics is preserved.

\section{Accuracy Scenario of Ontology Matching Tools}

In the accuracy scenario of ontology matching tools, based on the test data (expected alignment) and on the base measures of one test execution (output alignment), the following derived measures were specified:

- Alignment precision. The ratio between the output alignments returned by the tool that match expected alignments and all output alignments returned by the tool when performing an ontology matching task. 
- Alignment recall. The ratio between the output alignments returned by the tool that match expected alignments returned by the tool when performing an ontology matching task and the total number of expected alignments.

- Alignment F-measure. The aggregation measure of alignment precision and alignment recall.

- Alignment H-measure. The aggregation measure of alignment precision and alignment recall (special case of F-measure).

Previously defined derived measures give insight into the extent of accuracy of the alignment produced by the ontology alignment tool.

\subsection{Identifying Indicators}

In this step, base and/or derived measures were aggregated in order to obtain quality indicators, i.e., measures related to the whole evaluation. Table 5.1 summarizes the results obtained for each measure and each type of tool ${ }^{2}$.

Table 5.1: Number of measures obtained for semantic technologies based on the SEALS evaluation results.

\begin{tabular}{|l|l|l|l|}
\hline Tool type $\backslash$ Measure & Base & Derived & Indicators \\
\hline Ontology engineering tools & 7 & 20 & 9 \\
\hline Reasoning systems & 11 & 0 & 16 \\
\hline Ontology matching tools & 3 & 4 & 6 \\
\hline Semantic search tools & 10 & 10 & 17 \\
\hline Semantic web service tools & 4 & 7 & 7 \\
\hline Total & $\mathbf{3 4}$ & $\mathbf{4 1}$ & $\mathbf{5 4}$ \\
\hline
\end{tabular}

\section{Conformance Scenario of Ontology Engineering Tools}

From the derived measures in the conformance scenario of ontology engineering tools, the following indicators were obtained:

- Ontology language component support. Whether the tool fully supports an ontology language component.

\footnotetext{
${ }^{2}$ As some measures are repeated across the tools, the total number of measures can be different than the sum.
} 
- Ontology language component coverage. The ratio of ontology components that are shared by a tool internal model and an ontology language model.

- Ontology information change. The ratio of information additions or losses when importing and exporting ontologies.

Using only the base measures, the following indicator is obtained:

- Import/Export errors. The ratio of tool execution errors when importing and exporting ontologies.

\section{Accuracy Scenario of Ontology Matching Tools}

From the base and derived measures in the accuracy scenario of ontology matching tools, the following quality indicators were obtained:

- Average alignment precision. The average precision of the ontology matching tool.

- Average alignment recall. The average recall of the ontology matching tool.

- Average alignment F-measure. The average F-measure of the ontology matching tool.

- Average alignment H-measure. The average harmonic measure of the ontology matching tool.

- Ontology alignment time. The average amount of time needed for the alignment task.

- Ontology alignment errors. The ratio of tool execution errors when performing an ontology alignment task.

\subsection{Specifying Relationships Between Measures}

Relationships between measures have been identified in a formal way by defining the formulas used for obtaining all derived measures and indicators. All the formulas that are defined are completely consistent, i.e., all the formulas contain measures that are already specified. Finally, these formulas formally define the existing dependencies between the quality measures in the quality model. 


\section{Conformance Scenario of Ontology Engineering Tools}

The formulas for the Information added (5.1) and Information lost (5.2) derived measures calculate the structural differences between the origin ontology and final ontology in terms of triples, i.e., in terms of all the components of the two ontologies. Possible values for these two measures are null and concrete information in terms of triples:

$$
\text { Information added }= \begin{cases}\text { null } & \text { if final ontology }=\text { origin ontology } \\ \text { final ontology - origin ontology } & \text { if final ontology } \neq \text { origin ontology }\end{cases}
$$

$$
\text { Information lost }= \begin{cases}\text { null } & \text { if final ontology }=\text { origin ontology } \\ \text { origin ontology - final ontology } & \text { if final ontology } \neq \text { origin ontology }\end{cases}
$$

The formula for the Structurally equivalent (5.3) derived measure is a propositional logic formula and it uses the previously defined measures to determine whether there is a difference in the structure of the origin and final ontology. Possible values for this measure are true and false:

Structurally equivalent $=($ information added $=$ null $) \wedge($ information lost $=$ null $)$

The formula for the Semantically equivalent (5.4) derived measure calculates if the origin ontology entails the final ontology, and vice versa. Possible values for this measure are true and false:

Semantically equivalent $=$

$$
=(\text { final ontology } \models \text { origin ontology }) \wedge(\text { origin ontology } \models \text { final ontology })
$$

Finally, the propositional logic formula for the Conformance (5.5) derived measure observes the conformance of the process of importing and exporting an ontology, with true and false as possible values:

$$
\text { Conformance }=\text { structurally equivalent } \wedge \neg(\text { execution problem })
$$

Similarly, formulas for the rest of indicators obtained in the conformance scenario of ontology engineering tools have been defined: Ontology language component support (5.6) with possible values true and false, Ontology language component coverage (5.7) with possible values in the range from 0 to 100, Ontology information change (5.8) with possible values in the range from 0 to 100, and Import/Export errors (5.9) with 
possible values in the range from 0 to 100 .

Ontology language component support $=$

$$
=\left(\frac{\# \text { tests that contain the component } \mid(\text { conformance }=\text { true })}{\# \text { tests that contain the component }}=1\right)
$$

Ontology language component coverage $=$

$$
=\frac{\# \text { components in the ontology language } \mid(\text { component support }=\text { true })}{\# \text { components in the ontology language }} \times 100
$$

Ontology information change $=$

$$
\begin{gathered}
=\frac{\# \text { tests } \mid(\text { information added } \neq \text { null } \vee \text { information lost } \neq \text { null })}{\# \text { tests }} \times 100 \\
\text { Import } / \text { Export errors }=\frac{\# \text { tests } \mid(\text { execution problem }=\text { true })}{\# \text { tests }} \times 100
\end{gathered}
$$

In some cases, a measure can be obtained using more than one formula. For example, the Ontology information change measure could be also obtained using formula 5.10.

$$
\text { Ontology information change }=\frac{\# \text { tests } \mid(\text { structurally equivalent }=\text { false })}{\# \text { tests }} \times 100
$$

\section{Accuracy Scenario of Ontology Matching Tools}

Formulas were also specified for each derived measure of the accuracy scenario of ontology matching tools: Alignment precision (5.11), Alignment recall (5.12), Alignment F-measure (5.13), and Alignment H-measure (5.14). Possible values for all these measures are in the range from 0 to 1.

Alignment precision $=$

$$
\frac{\# \text { output alignments } \mid \text { (output alignment } \in \text { expected alignments) }}{\text { \# output alignments }}
$$


Alignment recall $=$

$$
\frac{\# \text { output alignments } \mid \text { (output alignment } \in \text { expected alignments) }}{\# \text { expected alignments }}
$$

Alignment F-measure $=$

$$
\begin{gathered}
\frac{\text { alignment precision } \times \text { alignment recall }}{(1-\alpha) \times \text { alignment precision }+\alpha \times \text { alignment recall }}, \alpha=[0 . .1] \\
\text { Alignment H-measure }=\frac{\text { alignment precision } \times \text { alignment recall }}{\text { alignment precision }+ \text { alignment recall }} \times 2
\end{gathered}
$$

Finally, equation 5.15 shows the formula for the Average alignment precision quality indicator of the accuracy scenario of ontology matching tools; the other indicators in this evaluation scenario are defined in an analogous manner. Possible values for this measure are in the range from 0 to 1 .

$$
\text { Average alignment precision }=\frac{\sum_{n} \text { alignment precision of the } n^{\text {th }} \text { test }}{\# \text { tests }}
$$

\subsection{Defining Domain-Specific Quality Sub-character- istics}

In this step, from the indicators previously identified, quality sub-characteristics that are affected by those indicators are defined. In some cases it was possible to reuse existing quality sub-characteristics but, in others, domain-specific ones had to be

defined. All the definitions for quality sub-characteristics in SemQuaRE are adapted from the ISO SQuaRE standard.

\section{Conformance Scenario of Ontology Engineering Tools}

In the conformance scenario of ontology engineering tools, based on the measures and analyses presented above, three quality sub-characteristics have been identified:

- Ontology language model conformance. Degree to which the knowledge representation model of the software product adheres to the knowledge representation model of an ontology language. It can be measured using: 
- Ontology language component coverage.

- Ontology language component support.

- Ontology processing accuracy. Degree to which a product or system provides the correct results with the needed degree of precision when processing ontologies. It can be measured using:

- Ontology information change.

- Ontology processing maturity. Degree to which a system, product or component meets needs for reliability while processing ontologies under normal operation. It can be measured using:

- Import/Export errors.

Figure 5.5 presents the base measures, derived measures, indicators, and quality sub-characteristics of the conformance evaluation for ontology engineering tools.

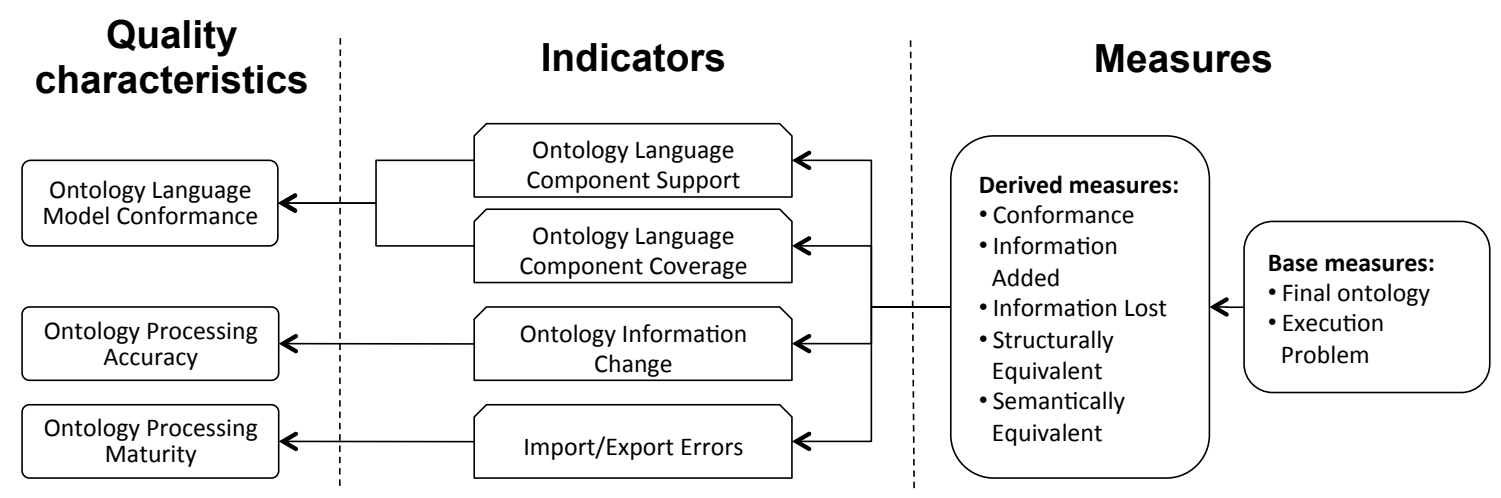

Figure 5.5: Entities in the conformance scenario of ontology engineering tools.

\section{Accuracy Scenario of Ontology Matching Tools}

In the accuracy scenario of ontology matching tools, three quality sub-characteristics have been identified:

- Ontology alignment accuracy. Degree to which a product or system provides the correct results with the needed degree of precision when performing an ontology alignment task. Ontology alignment accuracy is a sub-characteristic of Ontology processing accuracy and can be measured using:

- Average alignment precision. 
- Average alignment recall.

- Average alignment F-measure.

- Average alignment H-measure.

- Ontology alignment time behavior. Degree to which the response, processing times and throughput rates of a product or system meet requirements when performing an ontology alignment task. It can be measured using:

- Ontology alignment time.

- Ontology alignment maturity. Degree to which a system, product or component meets needs for reliability when performing an ontology alignment task under normal operation. Ontology alignment maturity is a sub-characteristic of Ontology processing maturity and can be measured using:

- Ontology alignment errors.

Figure 5.6 presents the base measures, derived measures, indicators, and quality sub-characteristics of the accuracy evaluation for ontology matching tools.

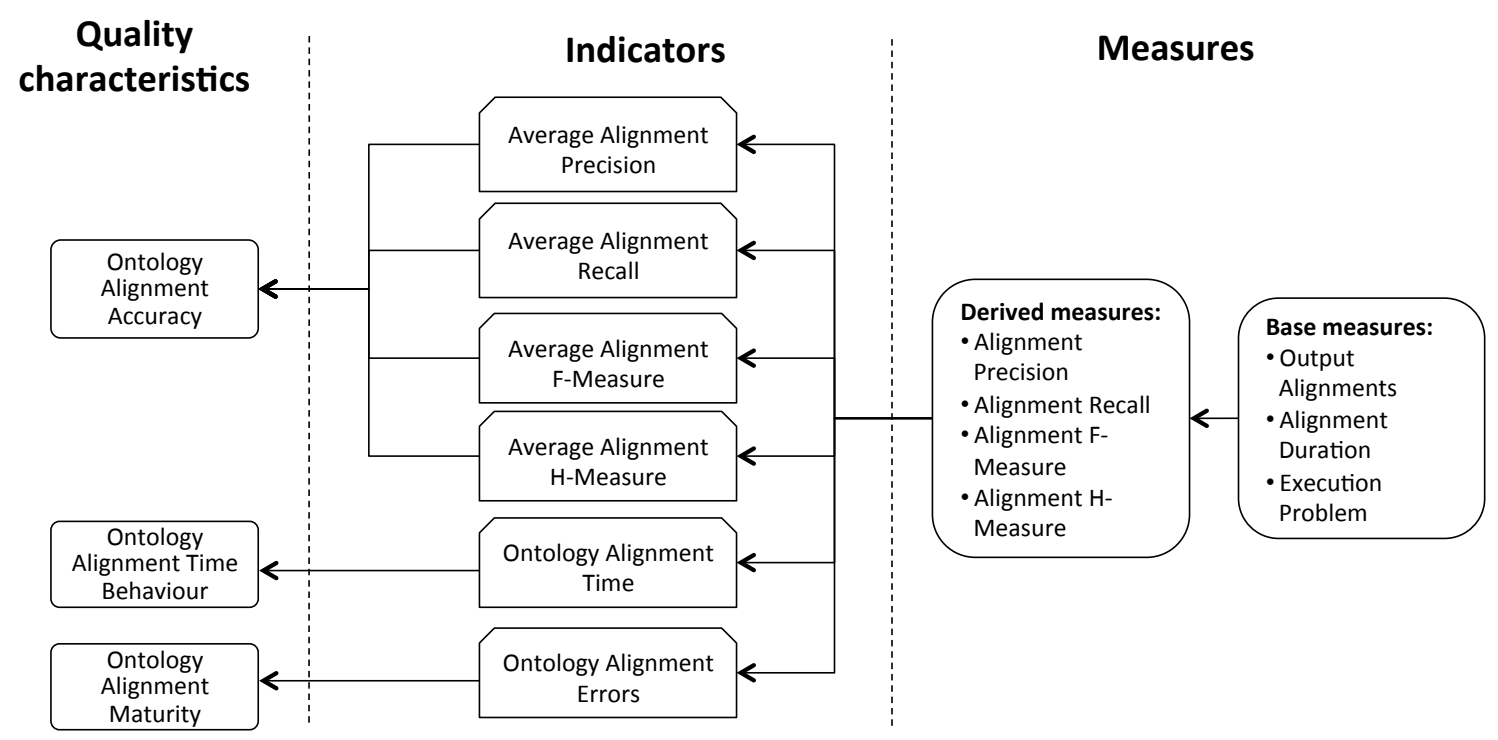

Figure 5.6: Entities in the accuracy scenario of ontology matching tools.

\section{SemQuaRE Sub-characteristics Overview}

In total, sixteen semantic quality sub-characteristics have been identified. Six of them are those described for the conformance and accuracy evaluation of ontology engineering tools and ontology matching tools, and the others are the following: 
- Ontology interchange accuracy. Degree to which a product or system provides the correct results with the needed degree of precision when interchanging ontologies (importing and exporting an ontology using two different tools).

- Ontology interchange maturity. Degree to which a system, product or component meets needs for reliability when interchanging ontologies (importing and exporting an ontology using two different tools) under normal operation.

- Ontology processing time behavior. Degree to which the response, processing times and throughput rates of a product or system meet requirements when working with ontologies.

- Ontology language model interoperability. Degree to which the knowledge representation model of the software product adheres to the knowledge representation model of an ontology language after interchanging ontologies (importing and exporting an ontology using two different tools).

- Reasoning accuracy. Degree to which a product or system provides the correct results with the needed degree of precision when performing a reasoning task.

- Reasoning time behavior. Degree to which the response, processing times and throughput rates of a product or system meet requirements when performing a reasoning task.

- Reasoning maturity. Degree to which a system, product or component meets needs for reliability when performing a reasoning task under normal operation.

- Semantic search accuracy. Degree to which a product or system provides the correct results with the needed degree of precision when performing a semantic search task.

- Semantic search time behavior. Degree to which the response, processing times and throughput rates of a product or system meet requirements when performing a semantic search task.

- Semantic web service discovery accuracy. Degree to which a product or system provides the correct results with the needed degree of precision when finding services that can be used to fulfill a given requirement from a service requester.

Besides these domain-specific quality sub-characteristics, the following general one can also be defined for semantic technologies:

- Ease of use. Degree to which a product or system is easy to operate and control by users. The indicator used to measure this sub-characteristic is Average usability value. 
Finally, those sub-characteristics that are contained into others have also been identified (e.g., Ontology alignment maturity is a sub-characteristic of Ontology processing maturity).

Table 5.2 shows all the semantic quality characteristics and the indicators that are defined for measuring them. For some quality indicators, such as those that measure semantic web service discovery accuracy, additional indicators related to a given number of retrieved documents (cutoff-point) can be obtained.

\subsection{Aligning Quality Sub-Characteristics with a Qual- ity Model}

As SQuaRE is a widely adopted and used quality model that has been by various authors extended to fit a particular domain [1, 12], it has been adopted for constructing the quality model for semantic technologies.

In the previous step, the set of quality sub-characteristics that are specific for semantic technologies has been identified. In this step, all the identified subcharacteristics were properly assigned to those sub-characteristics that already exist in the SQuaRE quality model, which are highly comprehensive as those in the ISO 9126 [8] one. For example, Reasoning time behavior is aligned to the Time behavior sub-characteristic, which is a sub-characteristic from SQuaRE that, by its definition, is highly related to Reasoning time behavior. Where needed, as suggested in [36], new sub-characteristics have been introduced to align existing ones with. An example of this is the Functional compliance sub-characteristic, which is introduced as a sub-characteristic of SQuaRE's Functional suitability characteristic.

Finally, some quality characteristics from SQuaRE related to quality in use are directly reused and are measured with indicators specified in one of the previous steps. These characteristics are Efficiency (which can be measured with Average answer found rate, Average number of attempts, and Average experiment time), and Satisfaction (which can be measured with Average satisfaction value).

Figure 5.7 shows a complete overview of SemQuaRE obtained from the SEALS evaluation results.

\subsection{Summary}

This chapter has presented the fourth contribution of this thesis, C4: SemQuaRE, a quality model for semantic technologies. SemQuaRE has been built using the bottom-up method described in Section 5.1, starting from the semantic technology evaluation results produced in the SEALS project. 
Table 5.2: Semantic quality sub-characteristics and indicators defined based on the SEALS evaluation results.

\begin{tabular}{|c|c|}
\hline $\begin{array}{l}\text { Semantic quality sub- } \\
\text { characteristic }\end{array}$ & Indicator \\
\hline $\begin{array}{l}\text { Ontology language model con- } \\
\text { formance }\end{array}$ & $\begin{array}{l}\text { Ontology language component support, Ontology language } \\
\text { component coverage }\end{array}$ \\
\hline $\begin{array}{l}\text { Ontology language model inter- } \\
\text { operability }\end{array}$ & $\begin{array}{l}\text { Ontology language component interoperability support, On- } \\
\text { tology language component interoperability coverage }\end{array}$ \\
\hline Ontology interchange accuracy & Interchange information change \\
\hline Ontology processing accuracy & Ontology information change \\
\hline $\begin{array}{l}\text { Ontology processing time behav- } \\
\text { ior }\end{array}$ & Ontology processing time, Average loading time \\
\hline Ontology processing maturity & Import/Export errors, Average successful loads \\
\hline Ontology interchange maturity & Ontology interchange errors \\
\hline Ontology alignment accuracy & $\begin{array}{l}\text { Average alignment precision, Average alignment recall, Aver- } \\
\text { age alignment F-measure, Average alignment H-measure }\end{array}$ \\
\hline $\begin{array}{l}\text { Ontology alignment time behav- } \\
\text { ior }\end{array}$ & Ontology alignment time \\
\hline Ontology alignment maturity & Ontology alignment errors \\
\hline Reasoning accuracy & $\begin{array}{l}\text { Classification correctness, Class satisfiability correctness, On- } \\
\text { tology satisfiability correctness, Entailment correctness, Non- } \\
\text { entailment correctness }\end{array}$ \\
\hline Reasoning time behavior & $\begin{array}{l}\text { Average classification time, Average class satisfiability time, } \\
\text { Average ontology satisfiability time, Average entailment time, } \\
\text { Average non-entailment time }\end{array}$ \\
\hline Reasoning maturity & $\begin{array}{l}\text { Classification errors, Class satisfiability errors, Ontology sat- } \\
\text { isfiability errors, Entailment errors, Non-entailment errors }\end{array}$ \\
\hline Semantic search accuracy & $\begin{array}{l}\text { Average search precision, Average search recall, Average } \\
\text { search F-measure, Number of completed queries, Average } \\
\text { number of results }\end{array}$ \\
\hline Semantic search time behavior & $\begin{array}{l}\text { Average query time, Average time per search result, Average } \\
\text { query input time, Maximum query input time, Average overall } \\
\text { question time }\end{array}$ \\
\hline $\begin{array}{l}\text { Semantic web service discovery } \\
\text { accuracy }\end{array}$ & $\begin{array}{l}\text { Average binary preference, Average discovery precision, Aver- } \\
\text { age discovery recall, Average discovery normalized discounted } \\
\text { cumulative gain, Average number of retrieved documents, } \\
\text { Average number of relevant documents retrieved, Average } \\
\text { reciprocal rank }\end{array}$ \\
\hline
\end{tabular}




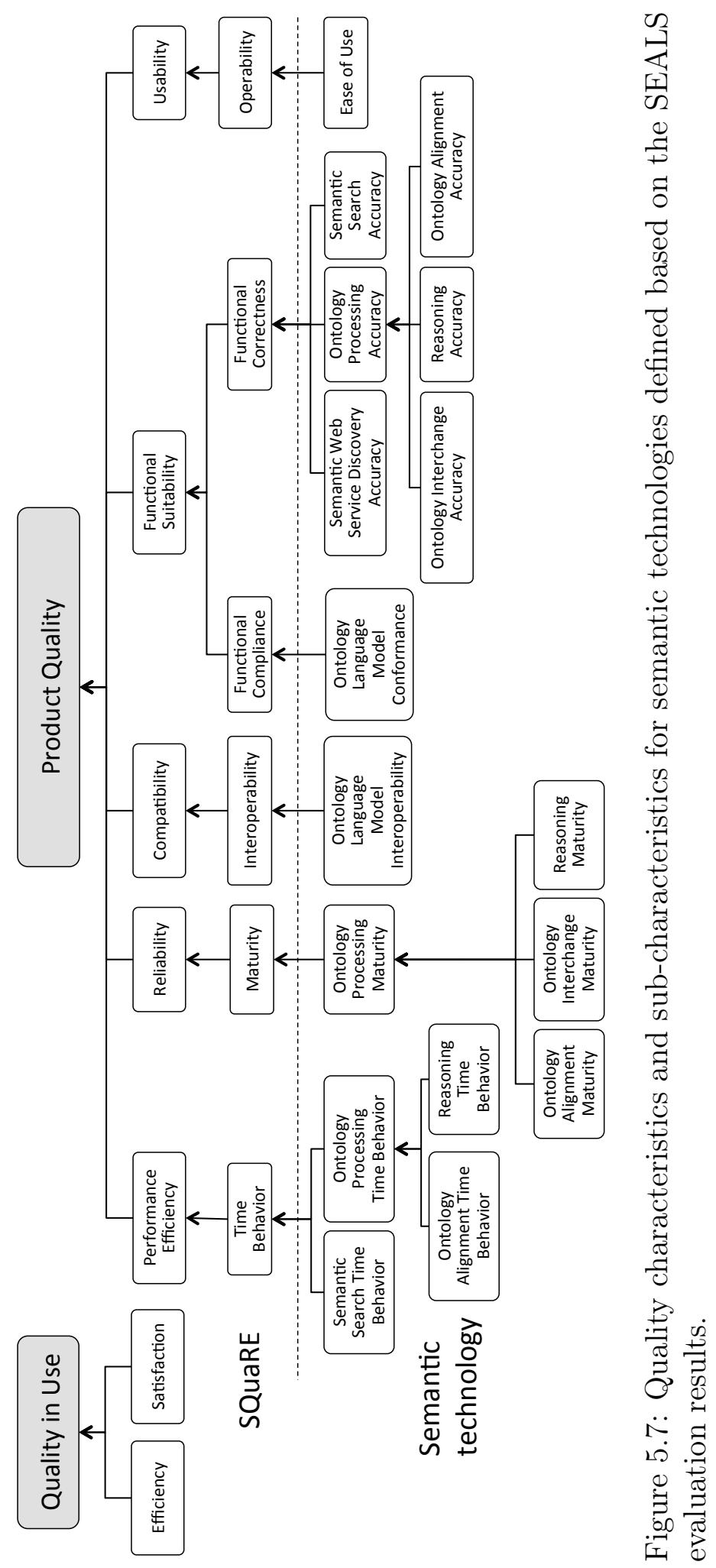


SemQuaRE describes a set of quality measures, which include base measures, derived measures and quality indicators related to semantic technologies. Where possible, for each of these measures a formula for its calculation was defined. Also, SemQuaRE describes a set of quality sub-characteristics that can be measured using previously mentioned quality measures, and a set of quality characteristics adopted from the SQuaRE standard.

To this extent, this chapter has presented the work carried out in this thesis in order to achieve the second objective, to apply the RIDER framework in the Semantic Web field for semantic technology recommendation using evaluation results. Although SemQuaRE is an important part, together with the work presented in the following chapter, of achieving this second objective, it is also an independent artefact related to quality specification of semantic technologies. 


\section{Chapter 6}

\section{RIDER for Semantic Technologies}

RIDER is a general recommendation framework that can be applied to any domain providing that the necessary artefacts are available, as described in detail in Section 4.7. Chapter 5 has presented one of such artefacts, which is the quality model for semantic technologies. This chapter presents the rest of the artefacts needed for the instantiation of RIDER in the semantic technology field which, together with the quality model for semantic technologies, form the constituent part of the RIDER recommendation framework in such field. In particular, the chapter describes:

- The semantic technology evaluation results that are used (Section 6.1).

- The ANP network in the semantic technology domain (Section 6.2).

- Expert pairwise comparisons of the criteria from the ANP network (Section $6.3)$.

Furthermore, this chapter also presents an illustrative example of using RIDER for semantic technology recommendation (Section 6.4) as well as the web application that supports such recommendation (Section 6.5).

\subsection{Semantic Technology Evaluation Results}

Evaluation results are an important part of the RIDER framework (Section 4.2) because the comparison of alternatives is performed automatically using the evaluation results and the comparison algorithms described in Section 4.3 and Section 4.4.

A corpus of semantic technology evaluation results has been produced in the SEALS project (Section 2.3.2), in which semantic technologies were automatically evaluated in a number of evaluation campaigns. Each evaluation campaign used predefined test data as input, and evaluated a number of tools, hence producing 
results for these tools. Evaluation results that were produced and stored in each evaluation contain base measures and derived measures (Section 2.2.5) and, in order to use these results in RIDER, several partners in the SEALS project were contacted with a request to provide them.

Since the evaluation results that were obtained from the SEALS project partners contained only base measures and derived measures, and since quality criteria in the RIDER recommendation process are related to quality indicators from a quality model (Section 4.1), these indicators had to be calculated from the obtained SEALS base and derived measures.

Figure 6.1 shows an overview of the process and data used for the calculation of quality indicators for semantic technologies. Every quality indicator was calculated from the SEALS evaluation results using the formulas in the SemQuaRE quality model (Appendix B), being each quality indicator related to one particular tool evaluated in a particular SEALS evaluation.

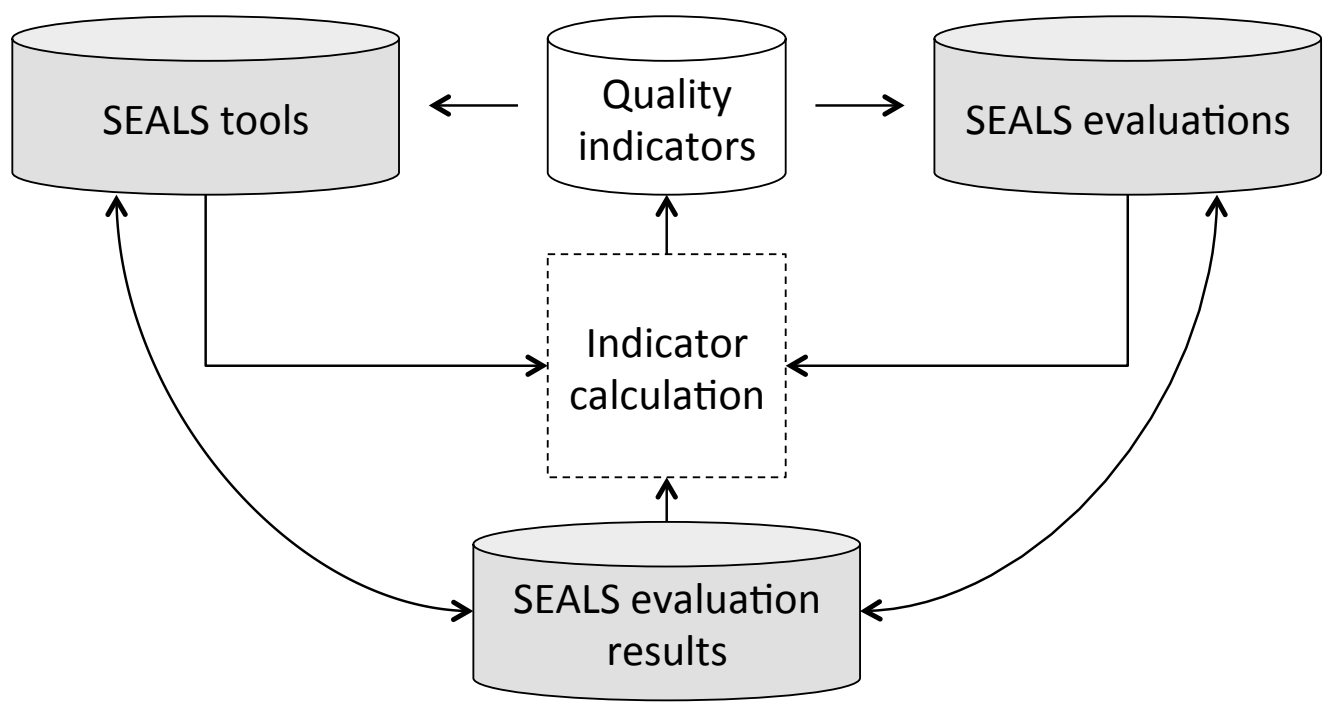

Figure 6.1: Calculation of quality indicators from the SEALS evaluation results.

Table 6.1 shows, for each type of tool, the quality indicators for which evaluation results were obtained from the second evaluation campaign in SEALS by combining the results presented in the project deliverables with raw results provided by the project partners. These indicators can be specified in user quality requirements and, as well as SemQuaRE quality model, are described using the Evaluation Result ontology (Section 4.2.1) and the Quality Model ontology (Section 4.1.1). Tables 6.2, $6.3,6.4,6.5$ and 6.6 show the tools and evaluation results for each quality indicator. 
Table 6.1: Quality indicators calculated from the SEALS evaluation results.

\begin{tabular}{|l|l|}
\hline Tool type & Indicators \\
\hline Ontology engineering tools [43] & Ontology language component coverage, Ontol- \\
ogy information change, Import/export errors, \\
Ontology language component interoperability \\
coverage, Interchange information change, On- \\
tology interchange errors, Ontology processing \\
time
\end{tabular}




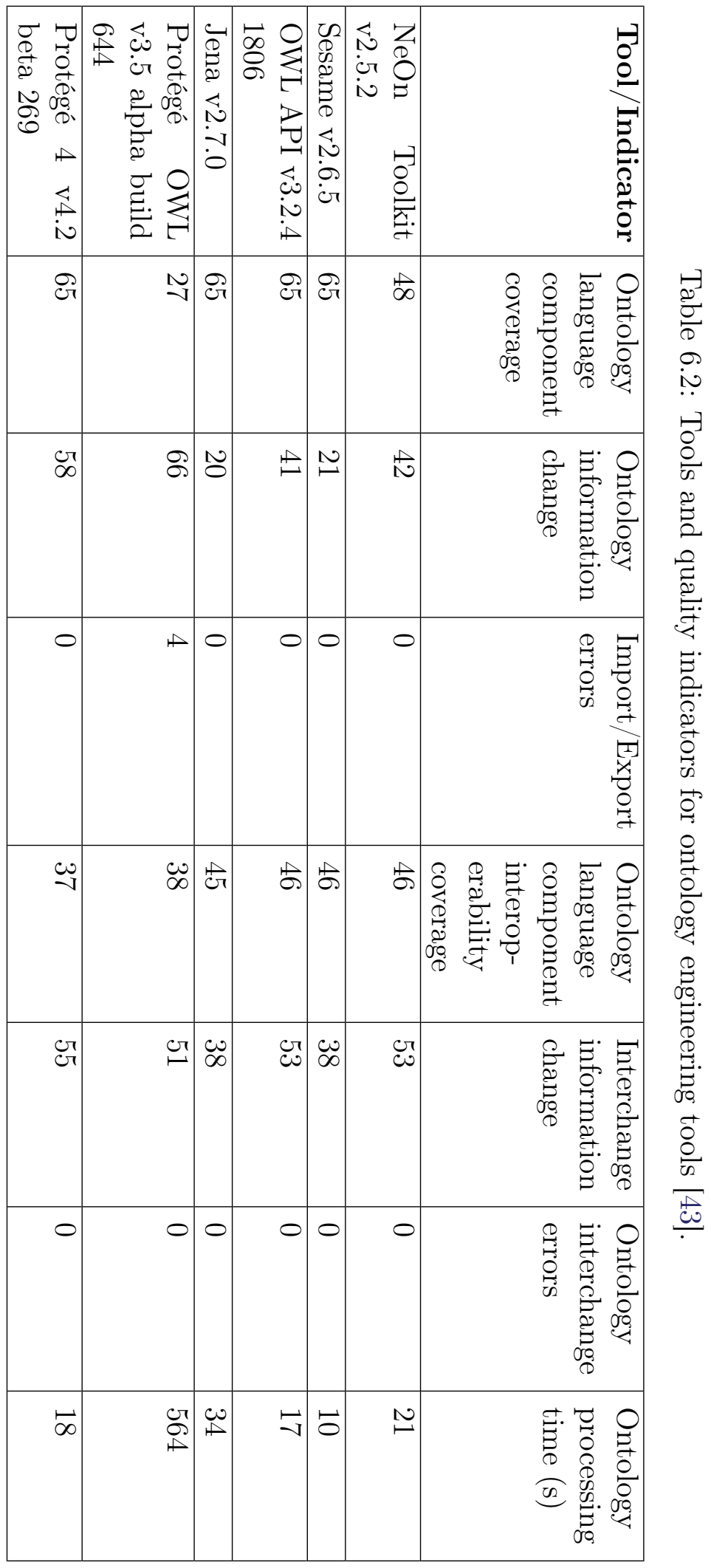


Table 6.3: Tools and quality indicators for reasoning systems [142].

\begin{tabular}{|l|l|l|}
\hline Indicator/Tool & Fact++ & Hermit \\
\hline Classification correctness & 64.5 & 66.5 \\
\hline Average classification time (ms) & 1708 & 950 \\
\hline Classification errors & 3 & 1 \\
\hline Class satisfiability correctness & 97 & 99 \\
\hline Average class satisfiability time (ms) & 296 & 200 \\
\hline Class satisfiability errors & 3 & 1 \\
\hline Ontology satisfiability correctness & 97 & 99 \\
\hline Average ontology satisfiability time (ms) & 298 & 204 \\
\hline Ontology satisfiability errors & 3 & 1 \\
\hline Entailment correctness & 4 & 98 \\
\hline Average entailment time (ms) & 4 & 77 \\
\hline Entailment errors & 15 & 0 \\
\hline Non-entailment correctness & 86 & 86 \\
\hline Average non-entailment time (ms) & 15 & 216 \\
\hline Non-entailment errors & 14 & 0 \\
\hline Average loading time (ms) & 215 & 207 \\
\hline
\end{tabular}

Table 6.4: Tools and quality indicators for ontology matching tools $[88]^{1}$.

\begin{tabular}{|l|l|l|l|l|l|}
\hline Tool/Indicator & $\begin{array}{l}\text { Average } \\
\text { alignment } \\
\text { precision }\end{array}$ & $\begin{array}{l}\text { Average } \\
\text { alignment } \\
\text { recall }\end{array}$ & $\begin{array}{l}\text { Average } \\
\text { alignment } \\
\text { F-measure }\end{array}$ & $\begin{array}{l}\text { Ontology } \\
\text { alignment } \\
\text { errors }\end{array}$ & $\begin{array}{l}\text { Ontology } \\
\text { alignment } \\
\text { time (s) }\end{array}$ \\
\hline LogMapLt & 0.89 & 0.5 & 0.64 & 0 & 13.5 \\
\hline Aroma & 0.91 & 0.66 & 0.76 & 0 & 27.25 \\
\hline GOMMA & 0.69 & 0.55 & 0.55 & 0 & 26.25 \\
\hline LogMap & 0.91 & 0.45 & 0.6 & 0 & 26.25 \\
\hline MapSSS & 0.98 & 0.68 & 0.8 & 0 & 66670 \\
\hline AUTOMS v2 & 0.97 & 0.54 & 0.69 & 0 & 63 \\
\hline YAM++ & 0.99 & 0.64 & 0.77 & 25 & 1750 \\
\hline WeSeE & 0.95 & 0.51 & 0.67 & 0 & 3968 \\
\hline Hertuda & 0.82 & 0.5 & 0.62 & 0 & 50.75 \\
\hline MaasMitch & 0.5 & 0.51 & 0.51 & 0 & 991 \\
\hline Lily & 0.93 & 0.58 & 0.71 & 25 & 4682 \\
\hline MapEVO & 0.14 & 0.1 & 0.11 & 0 & 16504 \\
\hline MapPSO & 0.25 & 0.08 & 0.12 & 25 & 554 \\
\hline
\end{tabular}


Table 6.5: Tools and quality indicators for semantic search tools [138].

\begin{tabular}{|l|l|l|l|l|l|l|}
\hline $\begin{array}{l}\text { Tool/ Indi- } \\
\text { cator }\end{array}$ & $\begin{array}{l}\text { Average } \\
\text { search } \\
\text { precision }\end{array}$ & $\begin{array}{l}\text { Average } \\
\text { search } \\
\text { recall }\end{array}$ & $\begin{array}{l}\text { Average } \\
\text { search } \\
\text { F-measure }\end{array}$ & $\begin{array}{l}\text { Average } \\
\text { loading } \\
\text { time } \\
\text { (ms) }\end{array}$ & $\begin{array}{l}\text { Average } \\
\text { number } \\
\text { of results }\end{array}$ & $\begin{array}{l}\text { Average } \\
\text { query } \\
\text { time } \\
\text { (ms) }\end{array}$ \\
\hline Arq v2.8.2 & 1 & 1 & 1 & 36415 & 324 & 369 \\
\hline Arq v2.9.0 & 1 & 1 & 1 & 37018 & 294 & 377 \\
\hline $\begin{array}{l}\text { rdfQuery } \\
\text { v0.5.1b- } \\
\text { FINAL }\end{array}$ & 0.99 & 0.99 & 0.99 & 247 & 236 & 37669 \\
\hline
\end{tabular}

Table 6.6: Tools and quality indicators for semantic web service tools [78].

\begin{tabular}{|l|l|l|}
\hline Indicator/Tool & $\begin{array}{l}\text { SAWSDL-OU } \\
\text { v.SAWSDL-OU }\end{array}$ & $\begin{array}{l}\text { WSMOLITE- } \\
\text { OU v.LITE-OU }\end{array}$ \\
\hline Average discovery precision & 0.75 & 0.7 \\
\hline Average discovery recall & 0.55 & 0.58 \\
\hline $\begin{array}{l}\text { Average discovery normalized dis- } \\
\text { counted cumulative gain }\end{array}$ & 0.61 & 0.57 \\
\hline $\begin{array}{l}\text { Average number of retrieved doc- } \\
\text { uments }\end{array}$ & 69 & 81 \\
\hline $\begin{array}{l}\text { Average number of relevant docu- } \\
\text { ments retrieved }\end{array}$ & 33 & 36 \\
\hline Average binary preference & 0.24 & 0.2 \\
\hline Average reciprocal rank & 0.95 & 0.87 \\
\hline
\end{tabular}

\subsection{ANP Network for Semantic Technologies}

The starting point in a recommendation process in RIDER is a model of a domain (Section 4.5), which can be obtained from the quality model by following the set of steps described in Section 4.1.

Starting from SemQuaRE (Chapter 5) and following the specified steps, the ANP network for semantic technologies has been constructed:

- Every quality indicator described in SemQuaRE has been introduced into the ANP network for semantic technologies as a network element. For example,

\footnotetext{
${ }^{1}$ Values for the Average alignment $H$-measure of the ontology matching tools obtained in the evaluation are the same as those for the Average alignment F-measure.
} 
Average alignment F-measure is an indicator and, therefore, it become an element in the network.

- Every quality sub-characteristic described in SemQuaRE has been introduced into the ANP network for semantic technologies as a network cluster. Then, the elements of the ANP network have been grouped into these clusters in such a way that an element is inside one particular cluster if the quality indicator represented by that element is used for measuring a quality sub-characteristic represented by that particular cluster. For example, Average alignment Fmeasure is an indicator that is used to measure the Ontology alignment accuracy sub-characteristic. Because of this, Average alignment F-measure has been defined as an element in the Ontology alignment accuracy cluster.

- Dependencies between the elements in the ANP network for semantic technologies have been deduced based on the formulas for obtaining the related quality indicators in SemQuaRE. For example, F-measure is calculated using the Precision and Recall derived measures and, therefore, the Average alignment F-measure indicator depends on the Average alignment precision and Average alignment recall. Furthermore, relying on expert opinions, a set of dependencies between ANP network elements has been completed.

- Dependencies between the clusters in the ANP network for semantic technologies have been deduced based on the dependencies between the elements. For example, since Average time per search result indicator from Semantic search time behaviour depends on Average number of results indicator from Semantic search accuracy, Semantic search time behaviour cluster depends on Semantic search accuracy.

Figure 6.2 presents the ANP network for semantic technologies with all the elements including clusters; dependencies between the elements are represented with arcs. The elements in the network represent criteria in a recommendation process, and these elements can be taken into account by the users when defining user quality requirements. Most of the elements and clusters belong to one semantic technology type; however, some elements and clusters are related to more than one type since related quality indicators and sub-characteristics from SemQuaRE also belong to more than one type. An example is the Average loading time element which is related to three types (reasoning systems, semantic search tools and semantic web service tools) and Ontology processing maturity which is related to two types (ontology engineering tools and semantic search tools). 


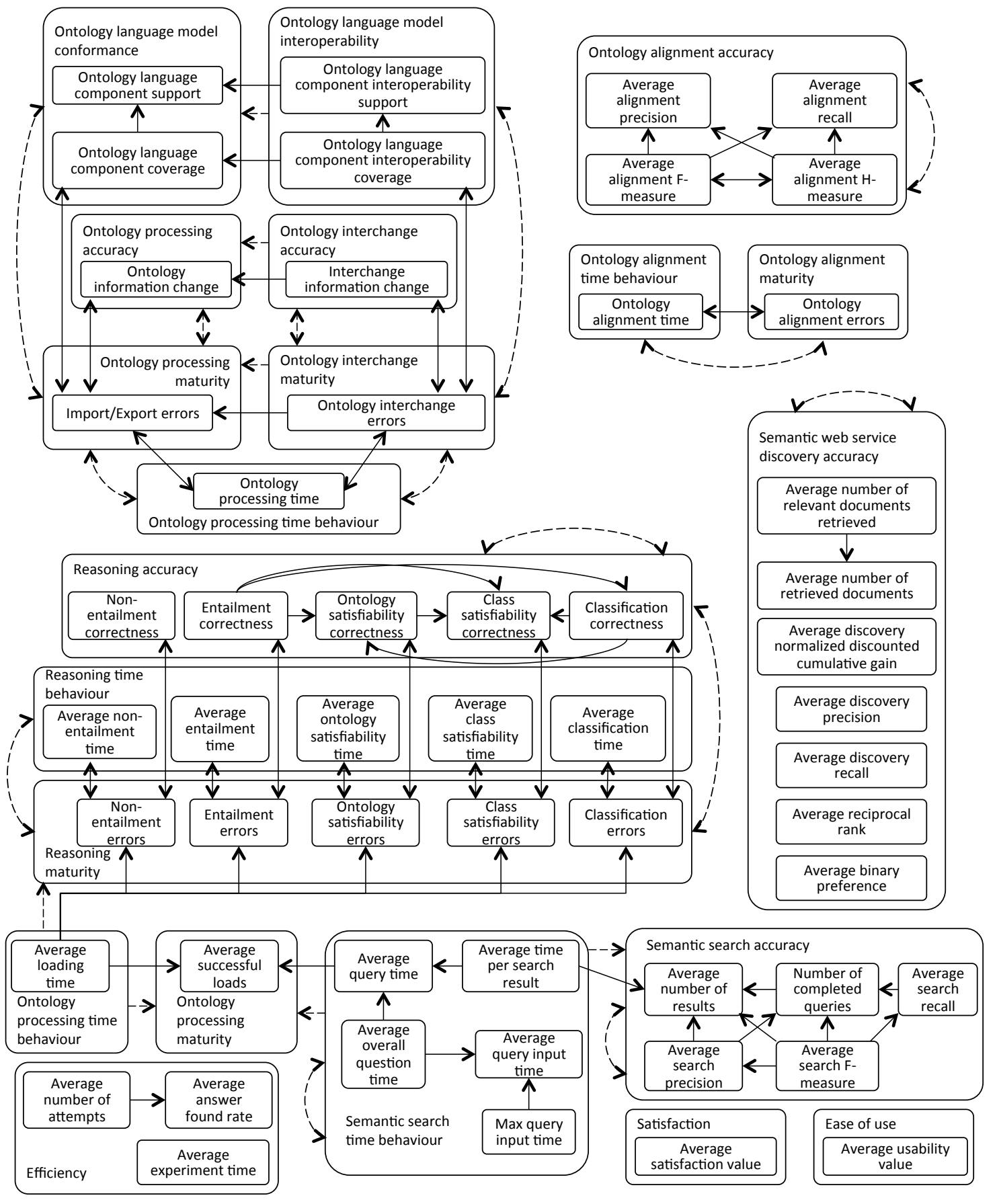

Figure 6.2: Semantic technology ANP network. 


\subsection{Pairwise Comparisons of Criteria}

Criteria pairwise comparisons are important because they are used for constructing the ANP supermatrix, as described in Section 4.6.

In the ANP network, if there is more than one element that belongs to the same cluster and that influences some other element in the network, it is necessary to calculate the influence priorities of these elements over the element they have influence on. This is done using the pairwise comparison technique (Section 2.1.4) and, based on the ANP network for semantic technologies, influence priorities for all the criteria were calculated.

This task, unlike the comparison of alternatives, was performed manually. Every two elements within a certain cluster that have influence on some other element have been compared by experts in a pairwise comparison with the following question: "given a dependent third element, which of the two elements has more influence?". These comparisons have been performed for all such elements in the ANP network for semantic technologies, as well as for all the clusters, and all comparisons are consensual. Next, all the pairwise comparisons of the criteria in the ANP network for semantic technologies are presented.

Table 6.7 shows the results of the pairwise comparison in which the priorities of indicators with respect to Average alignment F-measure (AAF) have been calculated. It can be seen from the network (Figure 6.2) that Average alignment F-measure depends on Average alignment H-measure (AAH), Average alignment precision (AAP), and Average alignment recall (AAR). Therefore, those three indicators have been compared through pairwise comparisons to determine their importance. For example, Average alignment precision has a strong plus over Average alignment H-measure which, according to the pairwise comparison scale (Section 2.1.4), implies the value 6 when Average alignment precision is compared to Average alignment $H$-measure, and the value $1 / 6$ when Average alignment $H$-measure is compared to Average alignment precision. Average alignment precision and Average alignment recall have equal importance with respect to Average alignment F-measure, which implies the value 1 in the comparison of these two indicators.

Table 6.7: Pairwise comparisons of indicators with respect to Average alignment F-measure

\begin{tabular}{|c|c|c|c|c|}
\hline $\begin{array}{c}\text { Average } \\
\text { alignment } \\
\text { F-measure }\end{array}$ & $\begin{array}{c}\text { Average } \\
\text { alignment } \\
\text { precision }\end{array}$ & $\begin{array}{c}\text { Average } \\
\text { alignment } \\
\text { recall }\end{array}$ & $\begin{array}{c}\text { Average } \\
\text { alignment } \\
\text { H-measure }\end{array}$ & Importance \\
\hline AAP & 1 & 1 & 6 & 0.462 \\
\hline AAR & 1 & 1 & 6 & 0.462 \\
\hline AAH & $1 / 6$ & $1 / 6$ & 1 & 0.076 \\
\hline
\end{tabular}


Column Importance gives the overall importance for each indicator. This comparison suggests that, e.g., Average alignment precision influences Average alignment F-measure with a 0.462 importance degree.

Table 6.8 shows the pairwise comparisons performed with respect to Average alignment H-measure.

Table 6.8: Pairwise comparisons of indicators with respect to Average alignment $H$-measure ${ }^{2}$.

\begin{tabular}{|c|c|c|c|c|}
\hline $\begin{array}{c}\text { Average } \\
\text { alignment } \\
\text { H-measure }\end{array}$ & $\begin{array}{c}\text { Average } \\
\text { alignment } \\
\text { precision }\end{array}$ & $\begin{array}{c}\text { Average } \\
\text { alignment } \\
\text { recall }\end{array}$ & $\begin{array}{c}\text { Average } \\
\text { alignment } \\
\text { F-measure }\end{array}$ & Importance \\
\hline AAP & 1 & 1 & 6 & 0.462 \\
\hline AAR & 1 & 1 & 6 & 0.462 \\
\hline AAF & $1 / 6$ & $1 / 6$ & 1 & 0.076 \\
\hline
\end{tabular}

Tables 6.9 and 6.10 show the pairwise comparisons performed with respect to Average search F-measure and Entailment correctness respectively.

Table 6.9: Pairwise comparisons of measures with respect to Average search Fmeasure ${ }^{3}$.

\begin{tabular}{|c|c|c|c|c|c|}
\hline $\begin{array}{c}\text { Average } \\
\text { search } \\
\text { F- } \\
\text { measure }\end{array}$ & $\begin{array}{c}\text { Average } \\
\text { number of } \\
\text { results }\end{array}$ & $\begin{array}{c}\text { Average } \\
\text { number of } \\
\text { completed } \\
\text { queries }\end{array}$ & $\begin{array}{c}\text { Average } \\
\text { search } \\
\text { precision }\end{array}$ & $\begin{array}{c}\text { Average } \\
\text { search } \\
\text { recall }\end{array}$ & Importance \\
\hline Av \# R & 1 & 1 & 6 & 6 & 0.429 \\
\hline Av \# CC & 1 & 1 & 6 & 6 & 0.429 \\
\hline ASP & $1 / 6$ & $1 / 6$ & 1 & 1 & 0.071 \\
\hline $\mathrm{ASR}$ & $1 / 6$ & $1 / 6$ & 1 & 1 & 0.071 \\
\hline
\end{tabular}

For some elements in the network, which depend on more than one other element from the same cluster, influence priorities are equal between all influencing elements because these influencing elements have equal importance. Those are:

- Average search precision, with 0.5 influence priority from Average number of results and Average number of completed queries.

\footnotetext{
${ }^{2} \mathrm{AAF}$ - Average alignment F-measure.

${ }^{3} \mathrm{Av} \# \mathrm{R}$ - Average number of results, AV \# CC - Average number of completed queries, ASP - Average search precision, ASR - Average search recall.
} 
Table 6.10: Pairwise comparisons of measures with respect to Entailment correctness ${ }^{4}$.

\begin{tabular}{|c|c|c|c|c|}
\hline $\begin{array}{c}\text { Entailment } \\
\text { correct- } \\
\text { ness }\end{array}$ & $\begin{array}{c}\text { Class satis- } \\
\text { fiability } \\
\text { correctness }\end{array}$ & $\begin{array}{c}\text { Ontology } \\
\text { satisfiability } \\
\text { correctness }\end{array}$ & $\begin{array}{c}\text { Classification } \\
\text { correctness }\end{array}$ & Importance \\
\hline CSC & 1 & 1 & 3 & 0.429 \\
\hline OSC & 1 & 1 & 3 & 0.429 \\
\hline CC & $1 / 3$ & $1 / 3$ & 1 & 0.143 \\
\hline
\end{tabular}

- Average loading time, with 0.2 influence priority from Classification errors, Ontology satisfiability errors, Class satisfiability errors, Entailment errors, and Non-entailment errors.

- Average overall question time, with 0.5 influence priority from Average query time and Average query input time.

- Classification correctness, with 0.5 influence priority from Class satisfiability correctness and Ontology satisfiability correctness.

Similar as for the quality indicators, a team of experts in semantic technologies performed the pairwise comparisons for the clusters in the semantic technology ANP network.

Tables 6.11 and 6.12 show the pairwise comparisons performed with respect to Ontology language model interoperability and Ontology interchange accuracy respectively.

Table 6.11: Pairwise comparisons of clusters with respect to Ontology language model interoperability ${ }^{5}$.

\begin{tabular}{|c|c|c|c|}
\hline $\begin{array}{c}\text { Ontology language } \\
\text { model } \\
\text { interoperability }\end{array}$ & $\begin{array}{c}\text { Ontology language } \\
\text { model conformance }\end{array}$ & $\begin{array}{c}\text { Ontology } \\
\text { interchange } \\
\text { maturity }\end{array}$ & Importance \\
\hline OLMC & 1 & 6 & 0.86 \\
\hline OIM & 0.166 & 1 & 0.14 \\
\hline
\end{tabular}

Tables 6.13 and 6.14 show the pairwise comparisons performed with respect to Semantic search time behavior and Ontology processing maturity respectively.

\footnotetext{
${ }^{4} \mathrm{CSC}$ - Class satisfiability correctness, OSC - Ontology satisfiability correctness, CC - Classification correctness.

${ }^{5}$ OLMC - Ontology language model conformance, OIM - Ontology interchange maturity.
} 
Table 6.12: Pairwise comparisons of clusters with respect to Ontology interchange accuracy ${ }^{6}$.

\begin{tabular}{|c|c|c|c|}
\hline $\begin{array}{c}\text { Ontology } \\
\text { interchange } \\
\text { accuracy }\end{array}$ & $\begin{array}{c}\text { Ontology processing } \\
\text { accuracy }\end{array}$ & $\begin{array}{c}\text { Ontology } \\
\text { interchange } \\
\text { maturity }\end{array}$ & Importance \\
\hline OPA & 1 & 6 & 0.86 \\
\hline OIM & 0.166 & 1 & 0.14 \\
\hline
\end{tabular}

Table 6.13: Pairwise comparisons of clusters with respect to Semantic search time behavior ${ }^{7}$.

\begin{tabular}{|c|c|c|c|c|}
\hline $\begin{array}{c}\text { Semantic } \\
\text { search time } \\
\text { behavior }\end{array}$ & $\begin{array}{c}\text { Ontology } \\
\text { processing } \\
\text { maturity }\end{array}$ & $\begin{array}{c}\text { Semantic } \\
\text { search } \\
\text { accuracy }\end{array}$ & $\begin{array}{c}\text { Semantic } \\
\text { search time } \\
\text { behavior }\end{array}$ & Importance \\
\hline OPM & 1 & 1 & 0.33 & 0.2 \\
\hline SSA & 1 & 1 & 0.33 & 0.2 \\
\hline SSTB & 3 & 3 & 1 & 0.6 \\
\hline
\end{tabular}

Table 6.14: Pairwise comparisons of measures with respect to Ontology processing maturity ${ }^{8}$.

\begin{tabular}{|c|c|c|c|c|}
\hline $\begin{array}{c}\text { Ontology } \\
\text { processing } \\
\text { maturity }\end{array}$ & $\begin{array}{c}\text { Ontology } \\
\text { language } \\
\text { model con- } \\
\text { formance }\end{array}$ & $\begin{array}{c}\text { Ontology } \\
\text { processing } \\
\text { accuracy }\end{array}$ & $\begin{array}{c}\text { Ontology } \\
\text { processing } \\
\text { time } \\
\text { behavior }\end{array}$ & Importance \\
\hline OLMC & 1 & 1 & 3 & 0.3 \\
\hline OPA & 1 & 1 & 6 & 0.6 \\
\hline OPTB & 0.33 & 0.16 & 1 & 0.1 \\
\hline
\end{tabular}

Tables 6.15 and 6.16 show the pairwise comparisons performed with respect to Reasoning accuracy and Ontology interchange maturity respectively.

Finally, Table 6.17 shows the pairwise comparisons performed with respect to Reasoning maturity.

${ }^{6} \mathrm{OPA}$ - Ontology processing accuracy.

${ }^{7}$ OPM - Ontology processing maturity, SSA - Semantic search accuracy, SSTB - Semantic search time behavior.

${ }^{8}$ OPTB - Ontology processing time behavior. 
Table 6.15: Pairwise comparisons of clusters with respect to Reasoning accuracy ${ }^{9}$.

\begin{tabular}{|c|c|c|c|}
\hline $\begin{array}{c}\text { Reasoning } \\
\text { accuracy }\end{array}$ & $\begin{array}{c}\text { Reasoning } \\
\text { accuracy }\end{array}$ & $\begin{array}{c}\text { Reasoning } \\
\text { maturity }\end{array}$ & Importance \\
\hline RA & 1 & 6 & 0.86 \\
\hline RM & 0.166 & 1 & 0.14 \\
\hline
\end{tabular}

Table 6.16: Pairwise comparisons of measures with respect to Ontology interchange maturity ${ }^{10}$.

\begin{tabular}{|c|c|c|c|c|c|}
\hline $\begin{array}{c}\text { Ontology } \\
\text { inter- } \\
\text { change } \\
\text { maturity }\end{array}$ & $\begin{array}{c}\text { Ontology } \\
\text { language } \\
\text { model } \\
\text { interoper- } \\
\text { ability }\end{array}$ & $\begin{array}{c}\text { Ontology } \\
\text { inter- } \\
\text { change } \\
\text { accuracy }\end{array}$ & $\begin{array}{c}\text { Ontology } \\
\text { processing } \\
\text { time } \\
\text { behavior }\end{array}$ & $\begin{array}{c}\text { Ontology } \\
\text { processing } \\
\text { maturity }\end{array}$ & Importance \\
\hline OLMI & 1 & 1 & 3 & 3 & 0.36 \\
\hline OIA & 1 & 1 & 6 & 3 & 0.41 \\
\hline OPTB & 0.33 & 0.16 & 1 & 0.33 & 0.08 \\
\hline OPM & 0.33 & 0.33 & 3 & 1 & 0.16 \\
\hline
\end{tabular}

Table 6.17: Pairwise comparisons of clusters with respect to Reasoning maturity ${ }^{11}$.

\begin{tabular}{|c|c|c|c|}
\hline $\begin{array}{c}\text { Reasoning } \\
\text { maturity }\end{array}$ & $\begin{array}{c}\text { Reasoning } \\
\text { accuracy }\end{array}$ & $\begin{array}{c}\text { Reasoning } \\
\text { time } \\
\text { behavior }\end{array}$ & Importance \\
\hline RA & 1 & 6 & 0.86 \\
\hline RTB & 0.166 & 1 & 0.14 \\
\hline
\end{tabular}

For some clusters in the network, which depend on more than one other cluster, influence priorities are equal between all influencing clusters because these influencing clusters have equal importance. This is the case for the Ontology processing time behaviour cluster, with 0.33 influence priority from Ontology processing maturity, Ontology interchange maturity, and Reasoning maturity. Finally, when comparing a cluster related to criteria to a cluster related to alternatives, with respect to a cluster related to criteria, equal importance is always assigned. Also, when comparing a

\footnotetext{
${ }^{9} \mathrm{RA}$ - Reasoning accuracy, RM - Reasoning maturity.

${ }^{10}$ OLMI - Ontology language model interoperability, OIA - Ontology interchange accuracy, OPTB - Ontology processing time behavior, OPM - Ontology processing maturity.

${ }^{11} \mathrm{RA}$ - Reasoning accuracy, RTB - Reasoning time behavior.
} 
cluster related to criteria with another cluster related to criteria, with respect to a cluster related to alternatives, equal importance is always assigned as well.

Using the method provided by Saaty for the calculation of consistency ratio of comparison matrix (Section 2.1.4), the consistency of every pairwise comparison in the ANP network for semantic technologies has been verified, including cluster comparisons. The consistency ratios were calculated for every pairwise comparison matrix, and the limit of 0.1 is satisfied in all the pairwise comparisons performed; the consistency ratio is zero in all the pairwise comparisons except to the ones related to Average search F-measure (Table 6.9), in which the consistency ratio is 0.02, Ontology processing maturity (Table 6.14), in which the consistency ratio is 0.05, and Ontology interchange maturity (Table 6.16), in which the consistency ratio is 0.04 .

\subsection{Using RIDER for Semantic Technology Recom- mendation}

This section describes a typical use case of RIDER for semantic technology recommendation.

Let us assume that a user needs to perform a task which consists in matching the concepts in some ontologies to other ontologies and, therefore, needs an ontology matching tool. Let us also assume that:

i) The user has two quality requirements: he or she wants a tool with at most 20 s runtime and with at most $20 \%$ error rate. These user quality requirements are specified in terms of a specific criterion (related to an element in the ANP network for semantic technologies presented in Figure 6.2) and a threshold. Specified user quality requirements are the only inputs required by the user in the whole recommendation process (Threshold column in Table 6.18).

ii) There are four possible ontology matching tools for performing this task $\left(A_{1}-A_{4}\right)$ for which evaluation results are described in Table 6.18.

Table 6.18: Evaluation results for the ontology matching tools use case.

\begin{tabular}{|c|c|c|c|c|c|}
\hline Criteria & $\mathbf{A}_{\mathbf{1}}$ & $\mathbf{A}_{\mathbf{2}}$ & $\mathbf{A}_{\mathbf{3}}$ & $\mathbf{A}_{\mathbf{4}}$ & Threshold \\
\hline Ontology alignment time & 14 & 32 & 10 & 28 & $20 \mathrm{~s}$ \\
\hline Ontology alignment errors & 30 & 25 & 13 & 9 & $20 \%$ \\
\hline
\end{tabular}

Based on these user quality requirements, the recommendations suggested by RIDER are obtained as follows: 
- Based on the specified user quality requirements and on the available evaluated alternatives, the ANP network for this specific use case is created based on the initial domain network (Figure 6.2), using one of the network extraction methods described in Section 4.5.

In this use case, the chosen extraction method of the ANP network is the one that produces a network that consists only of user quality requirements (Requirements network). The network produced in this example is presented in Figure 6.3, and it can be obtained automatically without the need for expert or user intervention.

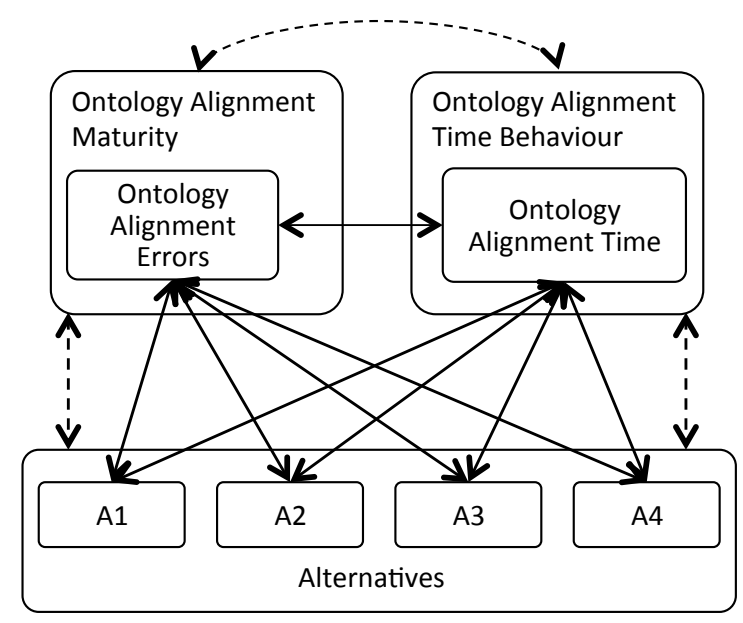

Figure 6.3: The ANP network for the ontology matching tools use case.

- Based on the extracted network, a supermatrix related to the particular use case is constructed. Table 6.19 shows the structure of a supermatrix related to the ontology matching tools use case.

Table 6.19: Supermatrix structure for the ontology matching tools use case ${ }^{12}$.

\begin{tabular}{|c|c|c|c|c|c|c|}
\hline $\begin{array}{c}\text { Network } \\
\text { elements }\end{array}$ & $\begin{array}{c}\text { Ontology } \\
\text { alignment } \\
\text { time }\end{array}$ & $\begin{array}{c}\text { Ontology } \\
\text { alignment } \\
\text { errors }\end{array}$ & A1 & A2 & A3 & A4 \\
\hline OAT & & & & & & \\
\hline OAE & & & & & & \\
\hline A1 & & & & & & \\
\hline A2 & & & & & & \\
\hline A3 & & & & & & \\
\hline A4 & & & & & & \\
\hline
\end{tabular}


- The supermatrix is filled with the comparison of alternatives, which is performed automatically using the comparison algorithms described in Section 4.4 and the evaluation results presented in Table 6.18; no additional input by users or experts is required for this task. In this use case, the chosen comparison algorithm is the Maximum distance algorithm with thresholds.

Table 6.20 and Table 6.21 show the comparison of alternatives with respect to Ontology alignment time and Ontology alignment errors, respectively.

Table 6.20: Comparison of the alternatives with respect to Average alignment precision using the Maximum distance algorithm with threshold.

\begin{tabular}{|c|c|c|c|c|c|}
\hline Alternatives & $\mathrm{A}_{1}$ & $\mathrm{~A}_{2}$ & $\mathrm{~A}_{3}$ & $\mathrm{~A}_{4}$ & Importance \\
\hline $\mathrm{A}_{1}$ & 1 & 9 & $1 / 2$ & 9 & 0.37 \\
\hline $\mathrm{A}_{2}$ & $1 / 9$ & 1 & $1 / 9$ & $1 / 2$ & 0.04 \\
\hline $\mathrm{A}_{3}$ & 2 & 9 & 1 & 9 & 0.53 \\
\hline $\mathrm{A}_{4}$ & $1 / 9$ & 2 & $1 / 9$ & 1 & 0.06 \\
\hline
\end{tabular}

Table 6.21: Comparison of the alternatives with respect to Ontology alignment errors using the Maximum distance algorithm with threshold.

\begin{tabular}{|c|c|c|c|c|c|}
\hline Alternatives & $\mathrm{A}_{1}$ & $\mathrm{~A}_{2}$ & $\mathrm{~A}_{3}$ & $\mathrm{~A}_{4}$ & Importance \\
\hline $\mathrm{A}_{1}$ & 1 & $1 / 3$ & $1 / 9$ & $1 / 9$ & 0.04 \\
\hline $\mathrm{A}_{2}$ & 3 & 1 & $1 / 9$ & $1 / 9$ & 0.07 \\
\hline $\mathrm{A}_{3}$ & 9 & 9 & 1 & $1 / 2$ & 0.37 \\
\hline $\mathrm{A}_{4}$ & 9 & 9 & 2 & 1 & 0.52 \\
\hline
\end{tabular}

Table 6.22 shows the supermatrix for the ontology matching tools use case, which is filled with the previously described pairwise comparison of alternatives.

- The supermatrix is filled with the criteria pairwise comparisons performed by experts when instantiating the RIDER framework, as well as with comparisons of criteria with respect to alternatives (Section 6.3). In this use case, since there are no elements in the ANP network that depend on more than one other element that belong to the same cluster (Figure 6.3), there is no need for criteria pairwise comparisons.

Table 6.23 shows the supermatrix filled with criteria dependencies.

\footnotetext{
${ }^{12}$ OAT - Ontology alignment time, OAE - Ontology alignment errors.
} 
Table 6.22: Supermatrix for the ontology matching tools use case filled with example alternatives comparison.

\begin{tabular}{|c|c|c|l|l|l|l|}
\hline $\begin{array}{c}\text { Network } \\
\text { elements }\end{array}$ & $\begin{array}{c}\text { Ontology } \\
\text { alignment } \\
\text { time }\end{array}$ & $\begin{array}{c}\text { Ontology } \\
\text { alignment } \\
\text { errors }\end{array}$ & A1 & A2 & A3 & A4 \\
\hline OAT & & & & & & \\
\hline OAE & & & & & & \\
\hline A1 & 0.37 & 0.04 & & & & \\
\hline $\mathrm{A} 2$ & 0.04 & 0.07 & & & & \\
\hline A3 & 0.53 & 0.37 & & & & \\
\hline A4 & 0.06 & 0.52 & & & & \\
\hline
\end{tabular}

Table 6.23: Supermatrix for the ontology matching tools use case filled with criteria dependencies.

\begin{tabular}{|c|c|c|c|c|c|c|}
\hline $\begin{array}{c}\text { Network } \\
\text { elements }\end{array}$ & $\begin{array}{c}\text { Ontology } \\
\text { alignment } \\
\text { time }\end{array}$ & $\begin{array}{c}\text { Ontology } \\
\text { alignment } \\
\text { errors }\end{array}$ & A1 & A2 & A3 & A4 \\
\hline OAT & $\mathbf{0}$ & $\mathbf{1}$ & $\mathbf{1}$ & $\mathbf{1}$ & $\mathbf{1}$ & $\mathbf{1}$ \\
\hline $\mathrm{OAE}$ & $\mathbf{1}$ & $\mathbf{0}$ & $\mathbf{1}$ & $\mathbf{1}$ & $\mathbf{1}$ & $\mathbf{1}$ \\
\hline $\mathrm{A} 1$ & 0.37 & 0.04 & $\mathbf{0}$ & $\mathbf{0}$ & $\mathbf{0}$ & $\mathbf{0}$ \\
\hline $\mathrm{A} 2$ & 0.04 & 0.07 & $\mathbf{0}$ & $\mathbf{0}$ & $\mathbf{0}$ & $\mathbf{0}$ \\
\hline $\mathrm{A} 3$ & 0.53 & 0.37 & $\mathbf{0}$ & $\mathbf{0}$ & $\mathbf{0}$ & $\mathbf{0}$ \\
\hline $\mathrm{A} 4$ & 0.06 & 0.52 & $\mathbf{0}$ & $\mathbf{0}$ & $\mathbf{0}$ & $\mathbf{0}$ \\
\hline
\end{tabular}

- Once the supermatrix is filled with alternatives and criteria pairwise comparisons, the cluster matrix is formed in an analogous way as the supermatrix. In this use case, there are three necessary comparisons: between Alternatives cluster and Ontology alignment time behaviour cluster with respect to Ontology alignment maturity cluster, between Alternatives cluster and Ontology alignment maturity cluster with respect to Ontology alignment time behavior cluster, and between Ontology alignment maturity cluster and Ontology alignment time behaviour cluster with respect to Alternatives cluster. In all three comparisons it is assumed that the two compared clusters are of equal importance (0.5). Table 6.24 shows the obtained cluster matrix.

- Finally, the limit supermatrix can be obtained using the supermatrix and the cluster matrix as described by the ANP method. The limit supermatrix is then

\footnotetext{
${ }^{13}$ OAM - Ontology alignment maturity, OATB - Ontology alignment time behavior.
} 
Table 6.24: Cluster matrix for the ontology matching tools use case ${ }^{13}$.

\begin{tabular}{|c|c|c|c|}
\hline Clusters & Alternatives & $\begin{array}{c}\text { Ontology } \\
\text { alignment } \\
\text { maturity }\end{array}$ & $\begin{array}{c}\text { Ontology } \\
\text { alignment } \\
\text { time } \\
\text { behavior }\end{array}$ \\
\hline Alternatives & 0 & 0.5 & 0.5 \\
\hline OAM & 0.5 & 0 & 0.5 \\
\hline OATB & 0.5 & 0.5 & 0 \\
\hline
\end{tabular}

used to rank the alternatives and provide a recommendation to the user for the specific use case.

Table 6.25 shows the limit supermatrix obtained in the use case. It can be observed that alternative $A 3$ is ranked as the first with the score of 0.15 , which is the only alternative that satisfies both user quality requirements.

Table 6.25: Limit supermatrix for the ontology matching tools use case.

\begin{tabular}{|c|c|c|c|c|c|c|}
\hline $\begin{array}{c}\text { Network } \\
\text { elements }\end{array}$ & $\begin{array}{c}\text { Ontology } \\
\text { alignment } \\
\text { time }\end{array}$ & $\begin{array}{c}\text { Ontology } \\
\text { alignment } \\
\text { errors }\end{array}$ & A1 & A2 & A3 & A4 \\
\hline OAT & 0.33 & 0.33 & 0.33 & 0.33 & 0.33 & 0.33 \\
\hline OAE & 0.33 & 0.33 & 0.33 & 0.33 & 0.33 & 0.33 \\
\hline A1 & 0.07 & 0.07 & 0.07 & 0.07 & 0.07 & 0.07 \\
\hline A2 & 0.02 & 0.02 & 0.02 & 0.02 & 0.02 & 0.02 \\
\hline A3 & 0.15 & 0.15 & 0.15 & 0.15 & 0.15 & 0.15 \\
\hline A4 & 0.1 & 0.1 & 0.1 & 0.1 & 0.1 & 0.1 \\
\hline
\end{tabular}

\subsection{Semantic Technology Recommendation System}

The generic RIDER web application (Section 4.8.2) has been instantiated in the semantic technology domain. It allows users to specify their quality requirements and get recommendations of the semantic technologies that best suite their needs.

The architecture of the semantic technology recommendation system as well as its dependencies are the same as those described in Section 4.8.2. The system is deployed online under http://www.development.seals-project.eu/seals-recommendation/.

The first page of the semantic technology recommendation system allows users to specify their desired quality requirements with respect to specific semantic technology types; these quality requirements are consistent with the indicators described in the 
quality model for semantic technologies (Section 5.4). Users can choose which quality indicators they want to take into account and for every desired quality indicator it is possible to specify a desired threshold. Also, by pointing the mouse on an icon additional information about the quality indicator is shown (Figure 6.4).

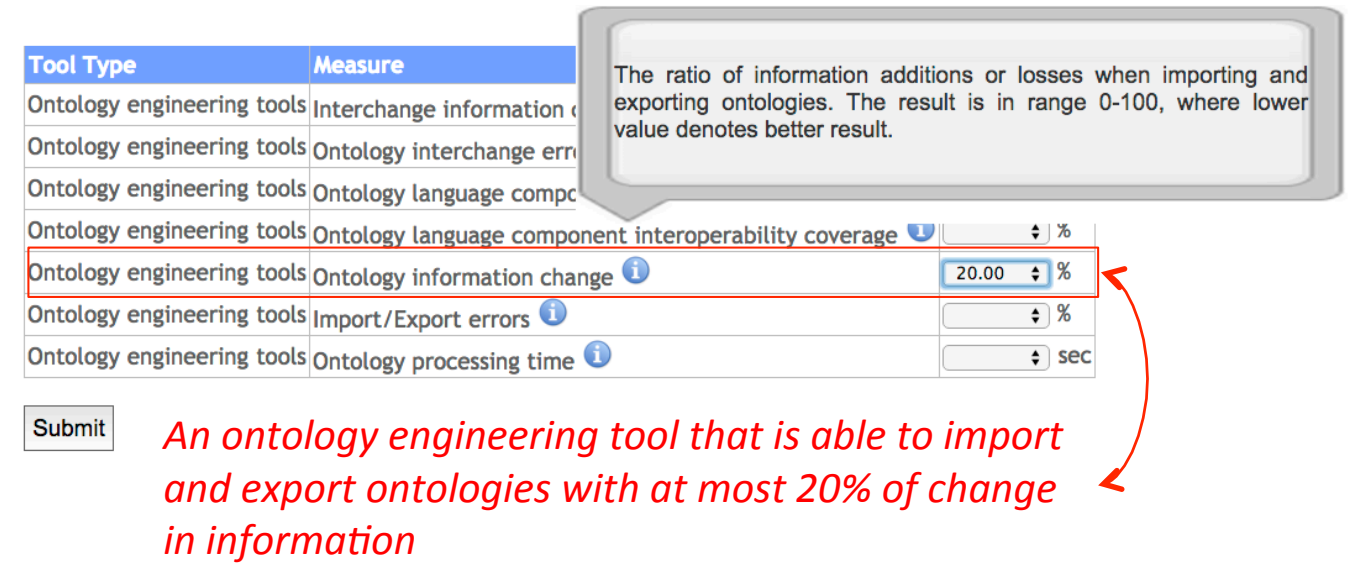

Figure 6.4: The first page of the semantic technology recommendation system.

Once the quality requirements are filled and the form is submitted, the results of the recommendation will be shown in the order of how well each of the alternatives (in this case semantic technologies) meet user quality requirements. For each alternative a tool is shown, together with the overall score obtained in the recommendation process.

Figure 6.5 shows and example of the results of the recommendation with respect to two quality requirements:

- Ontology language component coverage, with a threshold of 50.

- Ontology information change, with a threshold of 20.

In this example, each alternative consists of one ontology engineering tool, and these tools are listed for each alternative in the Tools column. For example, the alternative that is marked as Alternative 1 consists of the Jena 2.7.0 ontology engineering tool. Column Result shows the overall result that an alternative has obtained in a recommendation process, which is the result obtained in the limit supermatrix that is used for the final ranking of alternatives (Section 2.1.5). It can be observed that the first alternative has obtained the highest result in the recommendation process $(0.3874)$.

In order to give the user more details on the recommendation process and on the obtained ranking of alternatives, a separate table shows values for every quality 


\begin{tabular}{|l|l|l|}
\hline Order & Tools & Result \\
\hline Alternative 1 & Jena 2.7.0 & 0.3874 \\
\hline Alternative 2 & Sesame 2.6.5 & 0.227 \\
\hline Alternative 3 & OWL API 3.2.4 & 0.1613 \\
\hline Alternative 4 & Protege 4 & 0.135 \\
\hline Alternative 5 & NeOn Toolkit 2.5.2 & 0.0649 \\
\hline Alternative 6 & Protege OWL 3.5 & 0.0244 \\
\hline
\end{tabular}

Figure 6.5: Results for the recommendation.

indicator that was taken into account in the recommendation process as a quality requirement, according to each alternative. Indicators are sorted according to their importance, and values for those alternatives that do not satisfy the quality requirement with respect to a specific indicator are colored in red, while values for those alternatives that satisfy the quality requirement with respect to a specific indicator are colored in green. Furthermore, importance of each quality indicator that is specified as a quality requirement is shown in the Importance column.

Figure 6.6 shows evaluation results for alternatives with respect to the quality indicators that are specified as quality requirements in the previous example (Figure 6.5). It can be observed that the first alternative is the only one that satisfies all two quality requirements. Alternatives 2-4 satisfy one quality requirement (Ontology language component coverage) and also have the same result for that requirement. Furthermore, alternative 2 is better that alternative 3, which is better than alternative 4 with respect to the second requirement (Ontology information change), and this is the order in which they are ranked. Alternatives 5 and 6 satisfy none of the requirements, and alternative 5 is better with respect to both requirements.

\begin{tabular}{|l|l|l|l|l|l|l|l|l|}
\hline Quality Measure Importance Alternative 1 Alternative 2 & Alternative 3 & Alternative 4 & Alternative 5 & Alternative 6 \\
\hline $\begin{array}{l}\text { Ontology } \\
\text { language } \\
\text { component } \\
\text { coverage }\end{array}$ & 0.500 & 65 & 65 & 65 & 65 & 48 & 27 \\
$\begin{array}{l}\text { Ontology } \\
\text { information } \\
\text { change }\end{array}$ & 0.500 & 20 & 21 & 41 & 58 & 42 & 66 \\
\hline
\end{tabular}

Figure 6.6: Results for all the tools with respect to the quality requirements.

By clicking on a particular tool in a ranking of the alternatives (column Tools, Figure 6.5), a page with the tool description appears, listing all the quality indicators (Measure Name column) and the evaluation results obtained for these indicators (Value column). Figure 6.7 shows an example of all evaluation results for one 
Evaluation results obtained for Jena 2.7.0

\begin{tabular}{|l|l|}
\hline Measure Name & Value \\
\hline Ontology information change & 20 \\
\hline Import/Export errors & 0 \\
\hline Ontology interchange errors & 0 \\
\hline Ontology language component interoperability coverage & 45 \\
\hline Ontology processing time & 34 \\
\hline Interchange information change & 38 \\
\hline Ontology language component coverage & 65 \\
\hline
\end{tabular}

Figure 6.7: Results for a particular tool.

particular tool (Jena 2.7.0). It can be seen that, apart from the results related to the indicators specified as quality requirements (Ontology language component coverage and Ontology information change), results for all quality indicators related to Jena 2.7.0 can be observed.

Finally, a user has a possibility to observe in a separate page the part of the weighted supermatrix (Section 2.1.5) related to all the quality indicators (criteria) in a recommendation process, including those that are specified as quality requirements. The page shows the influence priorities among all the quality requirements that were previously defined, as well as their importance obtained in a recommendation process, and it can help experienced users with the knowledge of the ANP method to get more insight into influence priorities and dependencies between the indicators.

Figure 6.8 shows the part of the weighted supermatrix related to quality indicators in the previous example. In this example, it can be observed that specified user quality requirements are independent (zeroes in the table). Because of this, both requirements are of equal importance (0.5, column Importance, Figure 6.6).

\begin{tabular}{|c|c|c|c|}
\hline Influence/Dependence & Importance & Ontology language component coverage & Ontology information change \\
\hline $\begin{array}{l}\text { Ontology language } \\
\text { component coverage }\end{array}$ & 0.500 & 0 & 0 \\
\hline $\begin{array}{l}\text { Ontology information } \\
\text { change }\end{array}$ & 0.500 & 0 & 0 \\
\hline
\end{tabular}

Figure 6.8: Part of the weighted supermatrix related to quality indicators (criteria).

\subsection{Summary}

This chapter has presented an instantiation of the RIDER framework for the recommendation of semantic technologies. To this extent, this chapter has presented the 
work carried out in this thesis in order to achieve the second objective, to apply the RIDER framework in the Semantic Web field for semantic technology recommendation using evaluation results.

In the context of achieving the second objective, this chapter has presented the fifth contribution of this thesis, C5: The application of the RIDER framework in the semantic technology domain. The RIDER artefacts related to semantic technologies, including evaluation results, ANP network for semantic technologies and pairwise comparisons of criteria have been described, together with an illustrative example and a web application.

The web application for the recommendation of semantic technologies using RIDER relies on SEALS evaluation results, and provides recommendations of semantic technologies based on user quality requirements as inputs. 


\section{Chapter 7}

\section{Evaluation}

The main objective of this thesis is to contribute to the state of the art in the Multiple Criteria Decision Making field by providing a Multiple Criteria Decision Making framework that takes advantage of evaluation results of alternatives and of user quality requirements over such alternatives, and by applying this MCDM framework in the Semantic Web field for semantic technology recommendation.

Since evaluation is an important phase in every research or engineering process [31], this chapter presents the evaluation of the main contributions of this thesis (Section 3.2). In particular, this chapter presents:

- The evaluation of the comparison algorithm without threshold (Section 7.1).

- The evaluation of the comparison algorithm with threshold (Section 7.2).

- The evaluation of the model extraction methods (Section 7.3).

- The evaluation of the SemQuaRE quality model (Section 7.4).

- The evaluation of RIDER framework relying on user feedback (Section 7.5).

\subsection{Evaluation of the Comparison Algorithm with- out Thresholds}

Section 4.3 presented different variations of the comparison algorithm without the thresholds imposed by user quality requirements. In order to determine which of these variations would be the most appropriate to be used, an evaluation addressing the functional appropriateness of the algorithm according to $\mathrm{SQuaRE}^{1}$ has been performed.

\footnotetext{
${ }^{1}$ The degree to which the functions facilitate the accomplishment of specified tasks and objectives.
} 
The goal of this evaluation is to determine how each algorithm variation behaves with different distributions of evaluation results. To this end, this evaluation covers the part of the RIDER framework related to the comparison algorithm without user quality requirements (Figure 7.1), with a set of evaluation results as input and a set of comparisons as output.

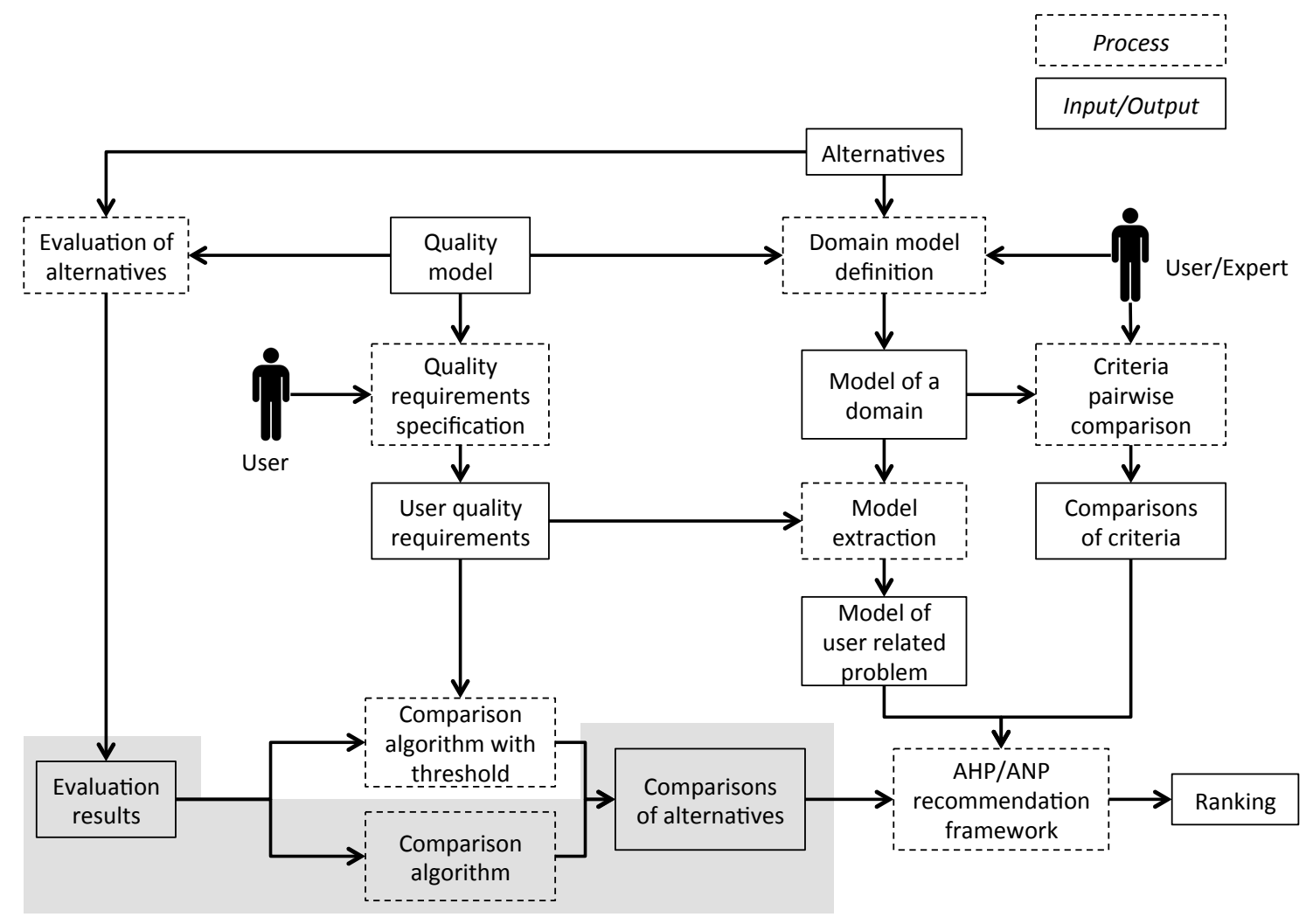

Figure 7.1: Evaluation of the comparison algorithm without thresholds.

For the purpose of this evaluation, the quality of a comparison algorithm is defined as its ability to produce a set of outputs that, for a given set of evaluation results, define the difference among the alternatives with a degree equivalent to the difference in the evaluation results. This means that the higher the difference between the two evaluation results, the higher the output of the algorithm has to be. Furthermore, in order to allow differentiating between different alternatives, it is expected from a good algorithm to produce outputs that cover all values on Saaty's scale.

Therefore, the quality of a comparison algorithm is determined by the following metrics:

- The number of different outputs $\left(N_{o}\right)$. There are nine possible outputs (from 1 to 9); the higher the number of outputs over a set of evaluation results denotes 
a better algorithm.

- The distance between the maximum and minimum output $\left(D_{o}\right)$. The maximum value for this indicator is eight; the higher the distance denotes a better algorithm.

The following hypotheses are made for this evaluation (Section 3.3):

- H1. The outputs of the algorithms for the comparison of alternatives that exploit evaluation results (in terms of $N_{o}$ and $D_{o}$ ) do not depend either on the distribution or the range (distance between the minimum and maximum values in the results) of such evaluation results.

- H2. The quality of the algorithms for the comparison of alternatives that exploit evaluation results (in terms of $N_{o}$ and $D_{o}$ ) does not improve when all the evaluation results are available.

In this evaluation, various datasets are used as inputs. Each of the datasets is used to produce the outputs of this evaluation, i.e., a set of numbers on Saaty's scale (Section 2.1.4) that represent comparisons in which every possible combination between any two results from a given dataset is compared in such a way that a better result is always compared to a worse one. The reason for this constraint is to avoid inconsistency in outputs and to ensure that all outputs are in the range 1-9, without reciprocal values.

\subsubsection{Evaluation Datasets}

Different use scenarios correspond to different sets of evaluation results. Therefore, several datasets containing integer numbers with different distributions within the range from zero to one hundred, in which a higher value denotes a better result, have been defined. The distributions used in the evaluation are chosen by taking into account well-known statistical distributions that can be expected to appear in evaluations as follows: i) the values can be found with equal probability along the range; ii) the values are clustered around a central value and are symmetrical; and iii) the values are clustered around a central value and are asymmetrical. These expectations can be satisfied by a uniform, normal, and Erlang distributions, respectively [23]. Therefore, for every dataset, one thousand results have been randomly generated with a Java program:

- Uniform dataset. This dataset was generated following a uniform distribution (i.e., results are equally distributed from lower to higher scale values). The minimum result generated was 0, the maximum 100 (Figure 7.2a) and the maximum distance between any two results is 100 while the minimum distance is 0 (i.e., two identical results). 


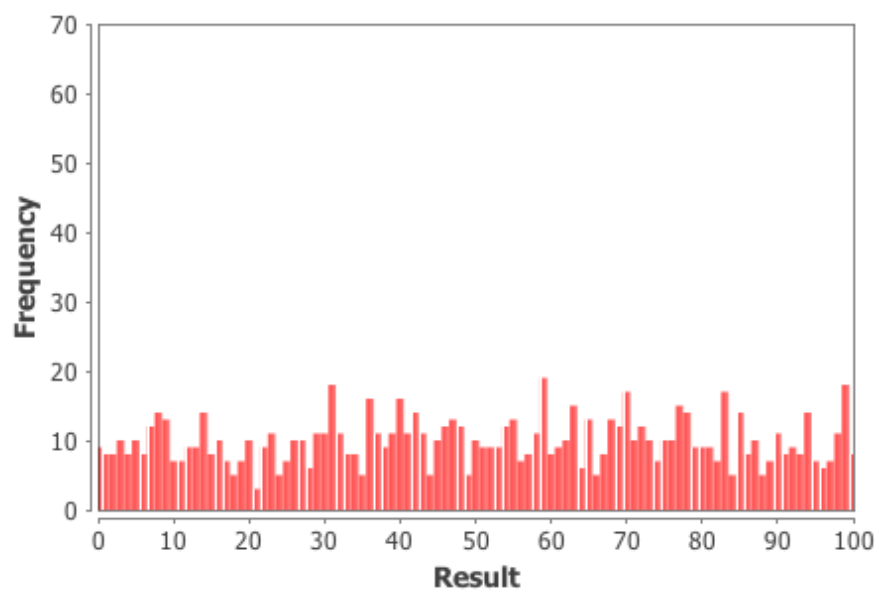

(a) Uniform dataset.

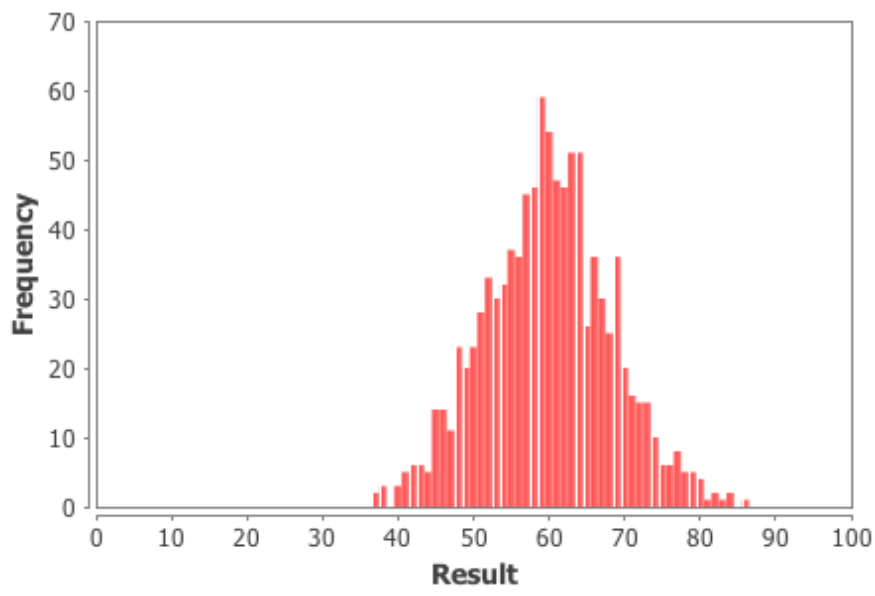

(b) Normal dataset.

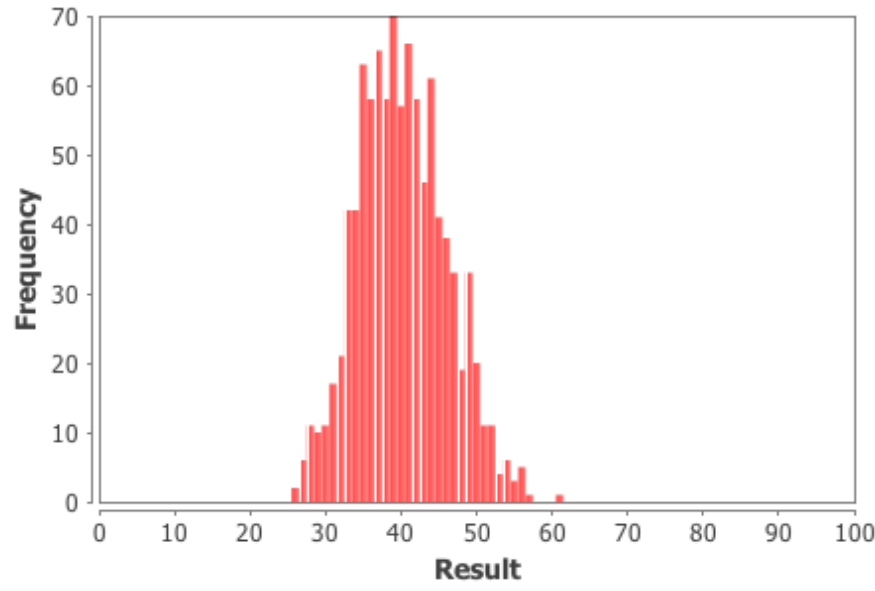

(c) Erlang dataset.

Figure 7.2: Evaluation datasets. 
- Normal dataset. This dataset was generated following a normal (Gaussian) distribution with a mean of 60 and a standard deviation of 8 . The minimum result generated was 37 , the maximum 86 (Figure $7.2 \mathrm{~b}$ ) and the maximum distance between any two results is 49 while the minimum distance is 0 .

- Erlang dataset. This dataset was generated following an Erlang distribution with a mean of 40 and a variance of 35 . The minimum result generated was 24 , the maximum 61 (Figure 7.2c) and the maximum distance between any two results is 37 while the minimum distance is 0 .

The number of results (in this case, one thousand) is chosen in order to cover all the values over the interval to which the results belong. This is to ensure that as less gaps as possible can be found in the results distribution.

Next, the results of the evaluation of each variation of the comparison algorithm for each evaluation dataset are presented.

\subsubsection{Uniform Dataset without Thresholds}

Figure 7.3a shows the behavior of the three variations of the comparison algorithm in the case of the Uniform dataset, where the horizontal axis represents the distance between the two results that are compared and the vertical axis represents the output in the comparison. Table 7.1 shows the quality metrics for each algorithm variation.

Table 7.1: Quality metrics for the different datasets without threshold.

\begin{tabular}{|c|c|c|l|}
\cline { 2 - 4 } \multicolumn{1}{c|}{} & Simple & Scale distance & Maximum distance \\
\cline { 2 - 4 } \multicolumn{1}{c|}{} & \multicolumn{3}{c|}{ Uniform dataset } \\
\hline $\mathrm{N}_{o}$ & 2 & 9 & 9 \\
\hline $\mathrm{D}_{o}$ & 8 & 8 & 8 \\
\hline \multicolumn{1}{|c|}{} & \multicolumn{3}{|c|}{ Normal dataset } \\
\hline $\mathrm{N}_{o}$ & 2 & 5 & 9 \\
\hline $\mathrm{D}_{o}$ & 8 & 4 & 8 \\
\hline & \multicolumn{3}{|c|}{ Erlang dataset } \\
\hline $\mathrm{N}_{o}$ & 2 & 4 & 9 \\
\hline $\mathrm{D}_{o}$ & 8 & 3 & 8 \\
\hline
\end{tabular}

The Simple algorithm produces only two outputs, which are the values of 1 and 9. This is the expected behavior for this algorithm because it distinguishes only the cases when two results are equal or when one result is better than the other.

The Scale distance algorithm and the Maximum distance algorithm behave identically (the figure shows how the lines of both algorithms coincide) and have the 


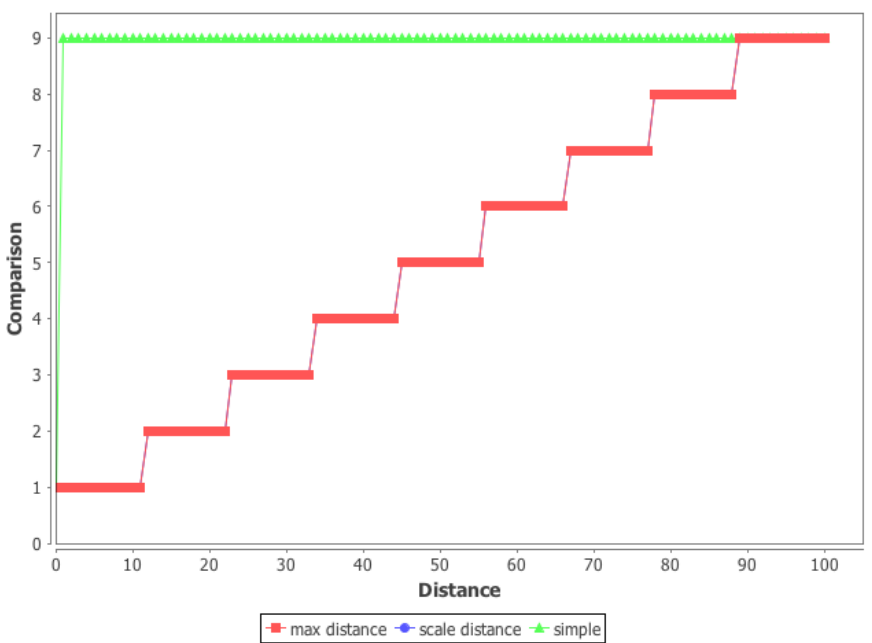

(a) Uniform dataset.

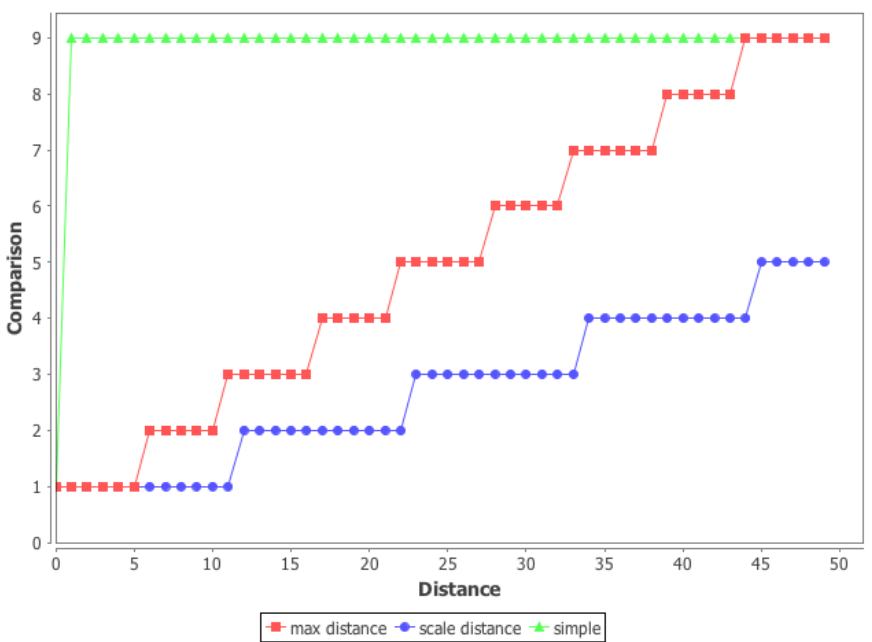

(b) Normal dataset.

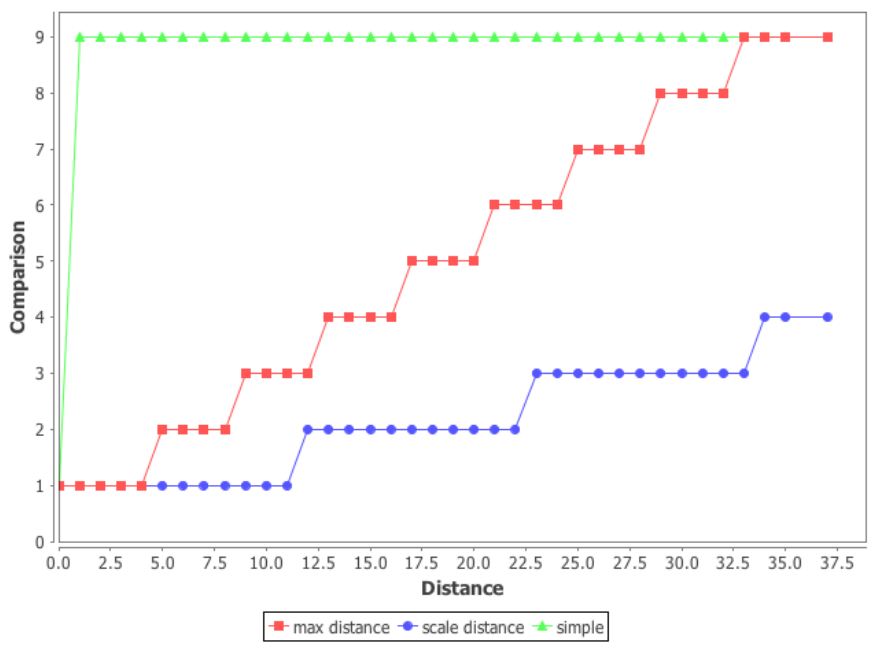

(c) Erlang dataset.

Figure 7.3: Comparisons with respect to result distances for different datasets without threshold. 
highest results with respect to both quality metrics. This is because the minimum and the maximum results in this dataset are equal to the minimum and the maximum values on the scale.

\subsubsection{Normal Dataset without Thresholds}

Figure $7.3 \mathrm{~b}$ shows the behavior of the three variations of the comparison algorithm in the case of the Normal dataset, where the horizontal axis represents the distance between the two results that are compared and the vertical axis represents the output in the comparison. Table 7.1 shows the quality metrics for each algorithm variation.

It can be observed that the Simple algorithm behaves the same as with the Uniform dataset. This is because both datasets contain results that are the same.

The Scale distance algorithm, unlike in the previous case, does not produce the outputs that are of higher values (i.e., 6, 7, 8, and 9). This is because the output intervals are calculated based on the complete scale, while the dataset contains only results that cover part of the scale (i.e., from 37 to 86).

The Maximum distance algorithm produces every possible output over the Saaty's scale, and has the best results with respect to both quality metrics. This is because the output intervals are calculated based on the results in a dataset, and not on the complete scale.

\subsubsection{Erlang Dataset without Thresholds}

Figure 7.3c shows the behavior of the three variations of the comparison algorithm in the case of the Erlang dataset, where the horizontal axis represents the distance between the two results that are compared and the vertical axis represents the output in the comparison. Table 7.1 shows the quality metrics for each algorithm variation.

It can be observed that the behavior of the three algorithms is similar to the previous case because of the same reasons. Besides, since the Erlang dataset covers a smaller range of results than the Normal one, the outputs of the Scale distance algorithm also cover a smaller range (i.e., $D_{o}$ is smaller).

\subsubsection{Discussion on the Evaluation without Thresholds}

From the analysis of the results of this evaluation, several conclusions can be outlined.

According to the defined quality metrics, the Maximum distance algorithm provides the best quality for both metrics in all the evaluations when compared to the two other algorithms.

The Maximum distance algorithm produces every output in the Saaty's scale, so all possible outputs are assigned in comparisons, which can be directly observed 
in the values of $N_{o}$ across evaluations (which are always the maximum number of outputs). This is because the output intervals in this algorithm are calculated based on the results in a dataset and not on the complete scale. However, the algorithm can only be applied when an access to all results is available. If it is not the case, another algorithm has to be applied.

Related to this, the Maximum distance algorithm always produces both the highest and the lowest output on the Saaty's scale, providing the maximum distance between the outputs in all the cases. This can be directly observed in the values of $D_{o}$ across evaluations.

The Scale distance algorithm differentiates less between two results if the dataset is more concentrated on one interval of the given scale. This can be observed in the lower distance between the maximum and the minimum output of this algorithm when comparing the Erlang dataset to the Normal dataset, where the Erlang dataset contains the results that cover a smaller scale interval than in the case of the Normal dataset.

The Simple algorithm always produces the maximum value for the distance between the maximum and the minimum output $\left(D_{o}\right)$ and always produces the minimum value for the number of outputs $\left(N_{o}\right)$. This is because it only distinguishes whether two results are the same or different.

In order to choose the algorithm to be applied in some specific case it is important to know whether the required inputs are available as well as to know the distribution and the range of the evaluation results. In the cases when the distribution and the range of the evaluation results are not known, the Maximum distance algorithm would be the most suitable to apply since it is independent on those characteristics and has better values for the quality metrics when compared to other algorithms. Furthermore, even when the distribution and the range of the evaluation results are known, the Maximum distance algorithm can be used before other algorithms since it showed the best values for quality metrics in all results distributions. However, while the Maximum distance algorithm tends to produce the output value of 1 for results that are very close, in these cases the Simple algorithm will give more importance to a better result.

With respect to the hypotheses formulated, the following conclusions can be drawn:

- H1. The outputs of the algorithms for the comparison of alternatives that exploit evaluation results (in terms of $N_{o}$ and $D_{o}$ ) do not depend either on the distribution or the range (distance between the minimum and maximum values in the results) of such evaluation results.

In the case of the Simple and the Maximum distance algorithms, the number of different outputs of the comparison algorithm $\left(N_{o}\right)$ and the distance between the 
maximum and minimum output $\left(D_{o}\right)$ are independent either of the distribution or the range of the evaluation results. However, in the case of the Scale distance algorithm, the number of outputs and the distance between the maximum and minimum output depend either on the distribution or the range of the evaluation results, which can be directly observed in the $N_{o}$ metric across the different evaluations. Therefore, this hypothesis is false and the number of different outputs of the comparison algorithm and the distance between the maximum and minimum output depend either on the distribution or the range of the evaluation results.

- H2. The quality of the algorithms for the comparison of alternatives that exploit evaluation results (in terms of $N_{o}$ and $D_{o}$ ) does not improve when all the evaluation results are available.

The results show how the quality of the comparison algorithm depends on its ability of distributing its outputs along every value in the Saaty's scale, and the best way to do so is when information about all the evaluation results is available (or at least the highest and the lowest evaluation results). The Maximum distance algorithm is the one that takes advantage of this information and, therefore, provides the best results for the quality metrics defined (with the maximum values for $N_{o}$ and $D_{o}$ ). Therefore, this hypothesis is false, and the quality of the comparison algorithm improves when all the evaluation results are available.

Furthermore, based on the results of the evaluation presented in this section and on the analysis of stated hypotheses, the overall conclusion is that the higher amount of evaluation results improves the quality of the comparison algorithms.

\subsection{Evaluation of the Comparison Algorithm with Thresholds}

Section 4.4 presented different variations of the comparison algorithm with thresholds (which are defined by user quality requirements) and, similarly as in the previous section, an evaluation addressing the functional appropriateness of the algorithm has been performed.

The goal of this evaluation is to determine the influence of thresholds on comparisons. To this end, this evaluation covers the part related to the comparison algorithm with the user quality requirements (Figure 7.4), with a set of evaluation results and a threshold extracted from the user quality requirements as inputs, and a set of comparisons as output.

The following hypotheses are made for this evaluation (Section 3.3): 


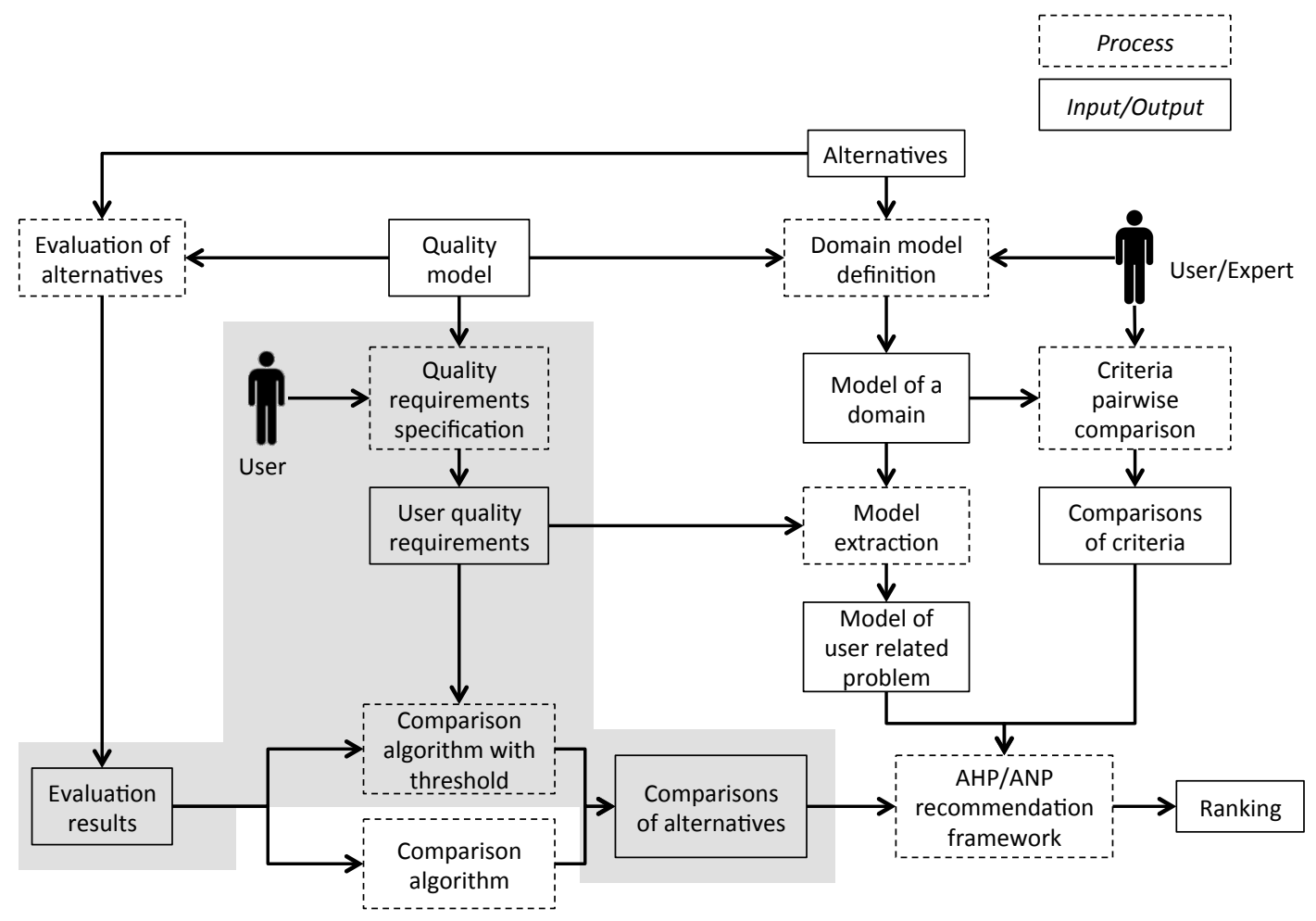

Figure 7.4: Evaluation of the comparison algorithm with thresholds.

- H3. User quality requirements (in terms of thresholds) do not influence the comparisons of alternatives performed by the algorithm.

- H4. User quality requirements (in terms of thresholds) do not help discriminating between alternatives.

The datasets used in this evaluation and the metrics for algorithm quality are the ones used in the evaluation of the comparison algorithm without thresholds (described in Section 7.1). The outputs are calculated identically as in the previous evaluation and, in all the cases, the threshold that is used in each dataset is determined as an arithmetic mean of the maximum and minimum result in a dataset.

Next, the results of the evaluation of each variation of the comparison algorithm for each evaluation dataset used are presented.

\subsubsection{Uniform Dataset with Thresholds}

Figure 7.5a shows the behavior of the three variations of the comparison algorithm in the case of the Uniform dataset when a threshold of 50 is introduced and Table 7.2 shows the quality metrics for each algorithm variation. 


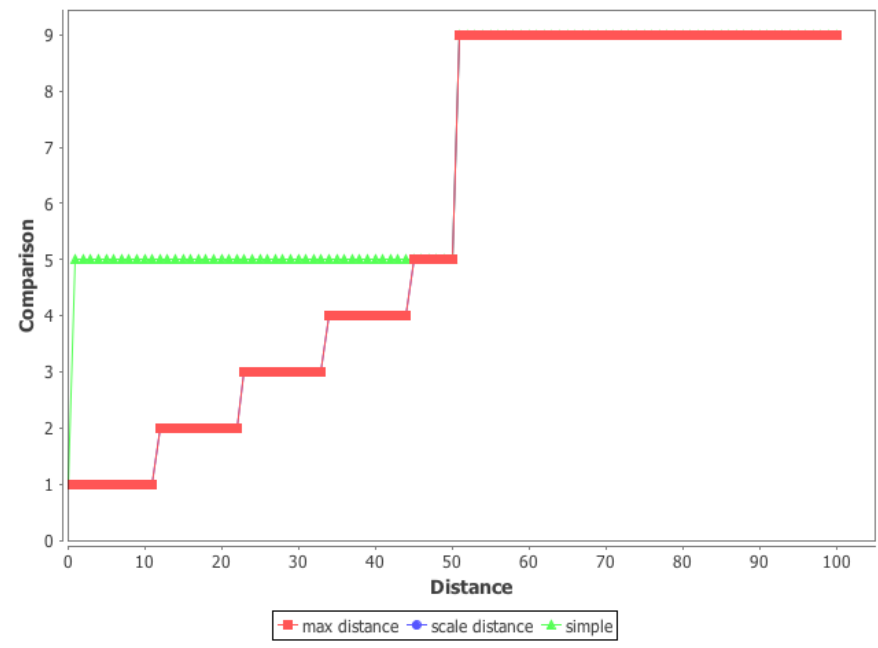

(a) Uniform dataset with threshold.

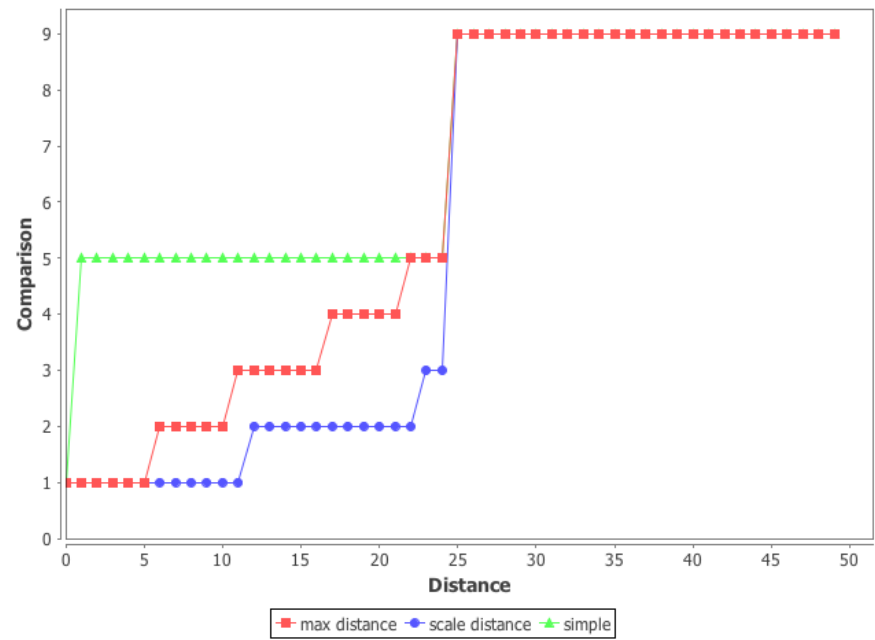

(b) Normal dataset with threshold.

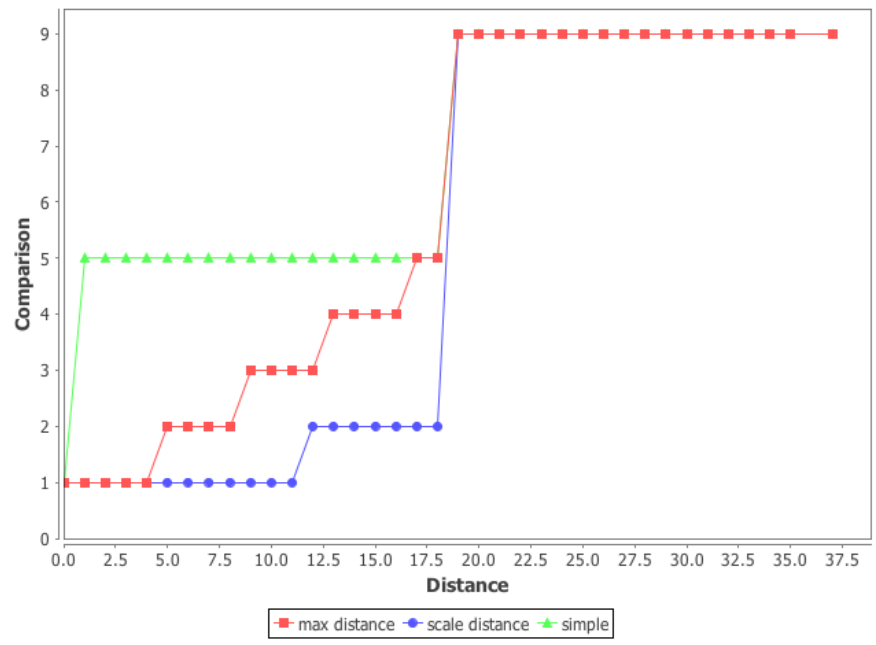

(c) Erlang dataset with threshold.

Figure 7.5: Comparisons with respect to result distances for different datasets with threshold. 
Table 7.2: Quality metrics for the different datasets with threshold.

\begin{tabular}{|c|c|c|c|}
\hline & Simple & Scale distance & Maximum distance \\
\hline & \multicolumn{3}{|c|}{ Uniform dataset } \\
\hline $\mathrm{N}_{o}$ & 3 & 6 & 6 \\
\hline \multirow{2}{*}{$\mathrm{D}_{o}$} & 8 & 8 & 8 \\
\hline & \multicolumn{3}{|c|}{ Normal dataset } \\
\hline $\mathrm{N}_{o}$ & 3 & 4 & 6 \\
\hline $\mathrm{D}_{o}$ & 8 & 8 & 8 \\
\hline & \multicolumn{3}{|c|}{ Erlang dataset } \\
\hline $\mathrm{N}_{o}$ & 3 & 3 & 6 \\
\hline $\mathrm{D}_{o}$ & 8 & 8 & 8 \\
\hline
\end{tabular}

It can be observed that, because of the threshold, the outputs of all three algorithms change significantly compared to the case without thresholds.

The Simple algorithm produces three outputs, and it is the maximum number of outputs that it is able to produce.

The Scale distance and the Maximum distance algorithms have identical behavior, as in the case of the Uniform dataset without threshold, because of the same maximum and minimum values in the results and in the scale. Furthermore, both algorithms produce six different outputs, which is less than in the case without threshold, and it can also be seen that the highest output values are 5 and 9 . This is because a threshold is introduced, which makes the higher distances in the comparison neglected, producing an output value of 9 when one result satisfies the threshold while the other does not.

\subsubsection{Normal Dataset with Thresholds}

Figure 7.5b shows the behavior of the three variations of the comparison algorithm in the case of the Normal dataset when a threshold of 62 is introduced and Table 7.2 shows the quality metrics for each algorithm variation.

Similarly as in the previous case, the outputs of all three algorithms change significantly because of the threshold. The Simple algorithm produces three outputs, as in the previous case; the highest outputs in the case of the Maximum distance algorithm are 5 and 9 and the highest outputs of the Scale distance algorithm are 3 and 9 because the results in this dataset do not include every possible value in the scale. 


\subsubsection{Erlang Dataset with Thresholds}

Figure 7.5c shows the behavior of the three variations of the comparison algorithm in the case of the Erlang dataset when a threshold of 43 is introduced and Table 7.2 shows the quality metrics for each algorithm variation.

The three algorithms behave the same as in the previous cases because of the introduced threshold. The Scale distance algorithm produces a fewer number of outputs $(1,2$, and 9); this is because the range of the Erlang results is smaller, as happened in the evaluation without thresholds.

\subsubsection{Discussion on the Evaluation with Thresholds}

From the analysis of the results of this evaluation, several conclusions can be outlined.

All the algorithms behave the same in the cases when only one result satisfies the threshold, assigning a value of 9 in the comparison of the better value to the worst one, and the behavior among algorithms is different only in the cases when both results satisfy the threshold or none of them satisfies it. Furthermore, when a threshold is introduced, until a certain distance between the two results from which all the outputs of each algorithm are 9 , for a specific distance all the algorithms produce two different outputs ${ }^{2}$, one being a value of 9 and another being a lower value. This is because the output depends not only on the distance, but on the introduced threshold and, while in some cases certain distance is defined by the two results of which only one satisfies the threshold (in this case a value of 9 is assigned as an output), in some cases the same distance is defined by the two results of which both satisfy the threshold (in this case a value lower than 9 is assigned as an output).

In the cases when a threshold is introduced, with respect to the $D_{o}$ metric all the algorithms behave the same (i.e., $D_{o}$ is 8 ). This is because all the algorithms recognize the cases when two identical results appear (with an output of 1) and when only one result satisfies the threshold (with an output of 9).

Similarly as in the case without a threshold, according to the defined quality metrics for the comparison algorithm (Section 7.1) the Maximum distance algorithm provides the best quality in all the evaluations when compared to the two other algorithms.

In the cases when two results do not satisfy a threshold or when two results do satisfy it, the algorithms behave the same as in the case without a threshold, and the Simple algorithm in most of the cases gives more importance to a better result than the two other algorithms. Furthermore, as in the case without threshold, while the Maximum distance algorithm tends to produce the output value of 1 for results that

\footnotetext{
${ }^{2}$ For the sake of better readability of the graphs, for those distances in Figure 7.5 that produce two output values, only values lower than 9 are shown.
} 
are very close, in these cases the Simple algorithm will give more importance to a better result.

With respect to the hypotheses, the following conclusions can be outlined:

- H3. User quality requirements (in terms of thresholds) do not influence the comparisons of alternatives performed by the algorithm.

Thresholds coming from user quality requirements influence the comparisons performed by the algorithm; this can be directly observed in the figures and by comparing the values of $N_{o}$ in the cases with and without threshold. Therefore, the hypothesis is false, and user quality requirements (in terms of thresholds) influence the comparisons performed by the algorithm, leading to differences in the number of outputs $\left(N_{o}\right)$.

- H4. User quality requirements (in terms of thresholds) do not help discriminating between alternatives.

Thresholds coming from user quality requirements help discriminating between results; by introducing a threshold two clusters of outputs are created (one with the lower outputs in the scale and another with outputs of 9), which can be directly observed in all three evaluations results. Therefore, the hypothesis is false.

\subsection{Evaluation of the Model Extraction Methods}

Section 4.5 presented several possible model extraction methods. In order to find the most suitable extraction method for recommending alternatives in terms of user quality requirements an evaluation has been performed.

This evaluation focuses on the ANP method, i.e., on a network as a model in a decision problem, and covers the part of the framework related to model extractions (Figure 7.6). It uses the ANP network, user quality requirements, evaluation results, comparison algorithm with threshold, and pairwise comparisons as inputs; the different alternative rankings that are obtained using different network extraction methods are the outputs. For this evaluation the Maximum distance algorithm is chosen because, as shown in the previous evaluation, it has the best behavior with respect to quality metrics and is independent of the dataset characteristics.

The evaluation of the network extraction methods addresses their functional appropriateness with the main goal of determining the most suitable extraction method for recommending alternatives in terms of user quality requirements. The following hypothesis is made with respect to this goal: 


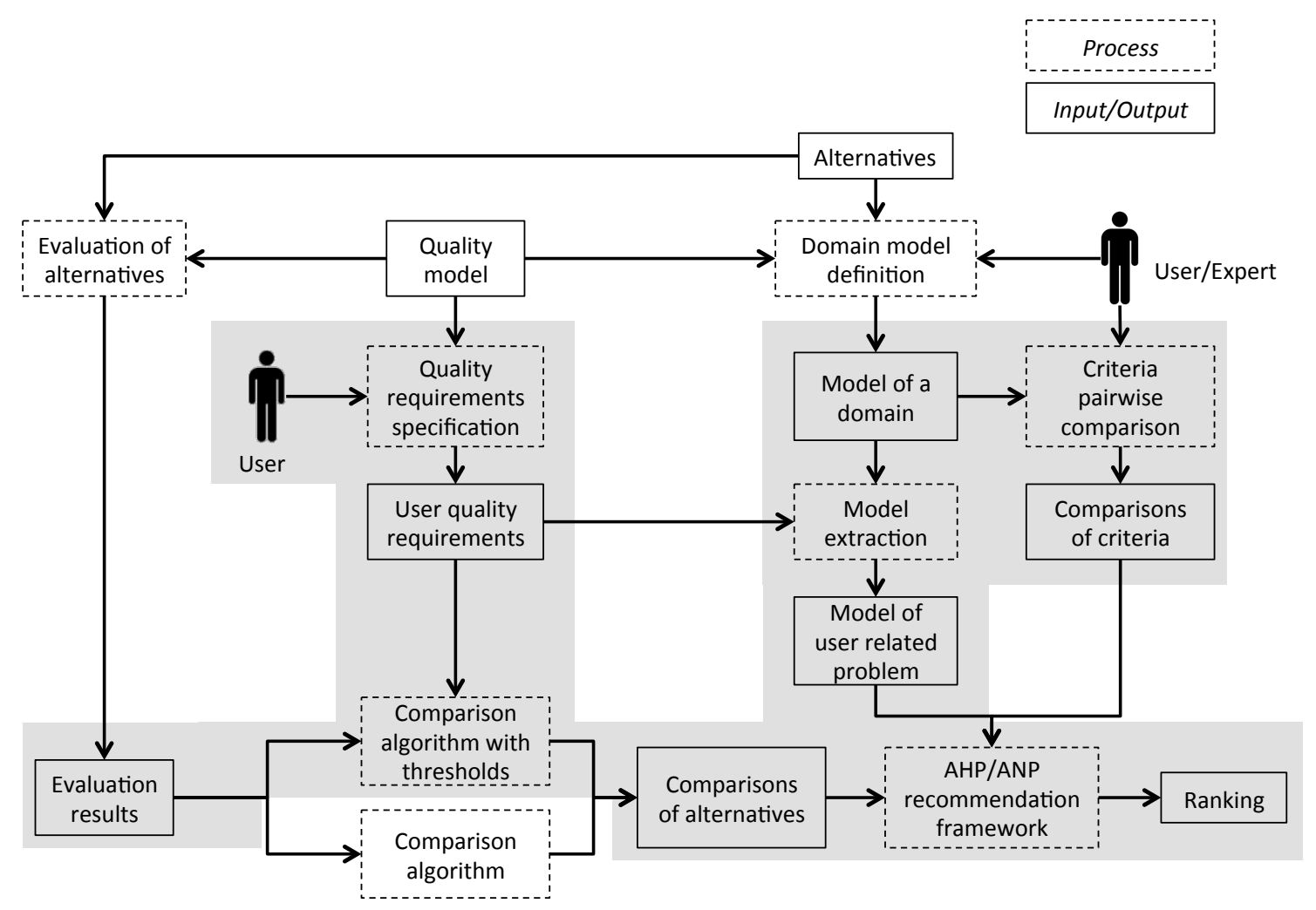

Figure 7.6: Evaluation of the model extraction methods.

- H5. Those alternatives that satisfy a higher number of specified user quality requirements are ranked better in an extracted model which only contains those criteria appearing in specified user quality requirements.

For the purpose of this evaluation, evaluation results that include seven criteria $\left(C_{1}-C_{7}\right)$ have been generated for six alternatives $(A-F)$ and have been used as inputs. These results have been generated randomly and are shown in Table 7.3; for the sake of simplicity, in all criteria a lower value denotes a better result. The ANP network for those criteria is shown on Figure 7.7 and the user quality requirements appear in Table 7.4, together with the number of quality requirements satisfied by each alternative for each set of quality requirements.

Table 7.5 shows the rankings obtained for each network extraction method and for each set of user quality requirements. For each set of user quality requirements the table shows the recommendations obtained using the Partial, Complete, and Requirements network methods (Section 4.5), differentiating between the cases where alternatives are compared only to criteria that appear in user quality requirements or to every criteria in the network (Comparisons column). Besides, the alternatives 
Table 7.3: Evaluation results used in the evaluation.

\begin{tabular}{|c|c|c|c|c|c|c|}
\cline { 2 - 7 } \multicolumn{1}{c|}{} & \multicolumn{6}{c|}{ Alternatives } \\
\hline Criterion & A & B & C & D & E & F \\
\hline $\mathrm{C}_{1}$ & 15 & 17 & 2 & 25 & 50 & 20 \\
\hline $\mathrm{C}_{2}$ & 15 & 12 & 25 & 35 & 65 & 35 \\
\hline $\mathrm{C}_{3}$ & 80 & 75 & 87 & 70 & 95 & 87 \\
\hline $\mathrm{C}_{4}$ & 10 & 10 & 2 & 15 & 30 & 15 \\
\hline $\mathrm{C}_{5}$ & 12 & 15 & 20 & 30 & 60 & 5 \\
\hline $\mathrm{C}_{6}$ & 75 & 75 & 85 & 70 & 95 & 90 \\
\hline $\mathrm{C}_{7}$ & 25 & 12 & 32 & 10 & 15 & 30 \\
\hline
\end{tabular}

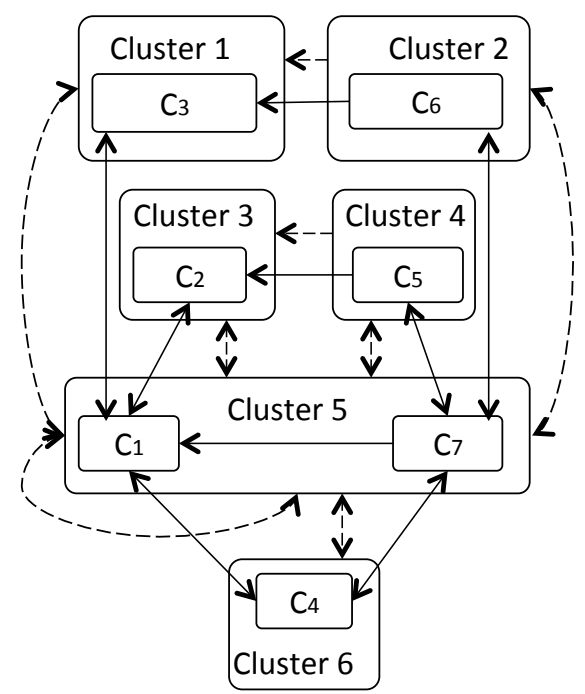

Figure 7.7: ANP network used in the evaluation.

that satisfy the highest number of quality requirements are marked in bold.

For illustration purposes, Figure 7.8 shows the Requirements (Figure 7.8a) and Partial (Figure 7.8b) networks for the first example in Table 7.5; the Complete network is that shown in Figure 7.7.

The baseline for the evaluation is the case where no user quality requirements are taken into account and the best alternative with respect to every criteria is searched; in this case the ranking is $B A D C F E$. From the analysis of Table 7.5 it can be seen that, comparing to the baseline, the ranking of alternatives changes when user quality requirements are introduced.

The extraction of an ANP network which consists only of the criteria specified as user quality requirements (Requirements network) tends to rank better those 
Table 7.4: User quality requirements and number of satisfying quality requirements.

\begin{tabular}{|c|c|c|c|c|c|c|c|}
\cline { 2 - 7 } \multicolumn{1}{c|}{} & $\begin{array}{c}\text { Quality } \\
\text { requirements }\end{array}$ & \multicolumn{5}{c|}{$\begin{array}{c}\text { Satisfied } \\
\text { requirements }\end{array}$} \\
\cline { 3 - 8 } \multicolumn{1}{c|}{} & $\mathbf{A}$ & $\mathbf{B}$ & $\mathbf{C}$ & $\mathbf{D}$ & $\mathbf{E}$ & $\mathbf{F}$ \\
\hline 1 & $\mathrm{C}_{1} \leq 20 ; \mathrm{C}_{5} \leq 10$ & 1 & 1 & 1 & 0 & 0 & 2 \\
\hline 2 & $\mathrm{C}_{3} \leq 85 ; \mathrm{C}_{5} \leq 10$ & 1 & 1 & 0 & 1 & 0 & 1 \\
\hline 3 & $\mathrm{C}_{4} \leq 10 ; \mathrm{C}_{6} \leq 80$ & 2 & 2 & 1 & 1 & 0 & 0 \\
\hline 4 & $\mathrm{C}_{6} \leq 80 ; \mathrm{C}_{7} \leq 20$ & 1 & 2 & 0 & 2 & 1 & 0 \\
\hline 5 & $\mathrm{C}_{1} \leq 15 ; \mathrm{C}_{3} \leq 85 ; \mathrm{C}_{5} \leq 10$ & 2 & 1 & 1 & 1 & 0 & 1 \\
\hline 6 & $\mathrm{C}_{1} \leq 15 ; \mathrm{C}_{2} \leq 15 ; \mathrm{C}_{3} \leq 80$ & 3 & 1 & 1 & 1 & 0 & 0 \\
\hline 7 & $\mathrm{C}_{4} \leq 5 ; \mathrm{C}_{6} \leq 80 ; \mathrm{C}_{7} \leq 10$ & 1 & 1 & 1 & 2 & 0 & 0 \\
\hline 8 & $\mathrm{C}_{1} \leq 10 ; \mathrm{C}_{3} \leq 75 ; \mathrm{C}_{7} \leq 13$ & 0 & 2 & 1 & 2 & 0 & 0 \\
\hline
\end{tabular}

Table 7.5: Evaluation of network extraction methods for the Maximum distance algorithm.

\begin{tabular}{|c|c|c|c|c|c|}
\hline & \multirow[b]{2}{*}{ User quality requirements } & \multirow[b]{2}{*}{ Comparisons } & \multicolumn{3}{|c|}{ ANP network extraction method } \\
\hline & & & Complete & Partial & Requirements \\
\hline \multirow[b]{2}{*}{1} & \multirow{2}{*}{$\mathrm{C}_{1} \leq 20 ; \mathrm{C}_{5} \leq 10$} & user criteria & CFABDE & CFABDE & \multirow{2}{*}{ FCABDE } \\
\hline & & all criteria & BACDFE & BACDFE & \\
\hline \multirow{2}{*}{2} & \multirow{2}{*}{$\mathrm{C}_{3} \leq 85 ; \mathrm{C}_{5} \leq 10$} & user criteria & DBAFCE & DBAFCE & \multirow{2}{*}{ FDBACE } \\
\hline & & all criteria & BADCFE & BADCFE & \\
\hline \multirow{2}{*}{3} & \multirow{2}{*}{$\mathrm{C}_{4} \leq 10 ; \mathrm{C}_{6} \leq 80$} & user criteria & CABDFE & CABDFE & \multirow{2}{*}{$\mathrm{ABCDFE}$} \\
\hline & & all criteria & BADCFE & DBCAFE & \\
\hline \multirow[b]{2}{*}{4} & \multirow{2}{*}{$\mathrm{C}_{6} \leq 80 ; \mathrm{C}_{7} \leq 20$} & user criteria & DBEACF & DBEACF & \multirow{2}{*}{ DBAECF } \\
\hline & & all criteria & BADCFE & DBCAFE & \\
\hline \multirow{2}{*}{5} & \multirow{2}{*}{$\mathrm{C}_{1} \leq 15 ; \mathrm{C}_{3} \leq 85 ; \mathrm{C}_{5} \leq 10$} & user criteria & ACDBFE & ACDBFE & \multirow{2}{*}{ FACDBE } \\
\hline & & all c & ABCDFE & ABCDFE & \\
\hline \multirow{2}{*}{6} & \multirow{2}{*}{$\mathrm{C}_{1} \leq 15 ; \mathrm{C}_{2} \leq 15 ; \mathrm{C}_{3} \leq 80$} & user criteria & ABDCFE & ABDCFE & \multirow{2}{*}{$\mathrm{ABCDFE}$} \\
\hline & & all criteria & ABDCFE & ABCDFE & \\
\hline \multirow{2}{*}{7} & \multirow{2}{*}{$\mathrm{C}_{4} \leq 5 ; \mathrm{C}_{6} \leq 80 ; \mathrm{C}_{7} \leq 10$} & user criteria & CDBAFE & CDBAFE & \multirow{2}{*}{ DBCAEF } \\
\hline & & all criteria & BCDAFE & DCBAFE & \\
\hline \multirow{2}{*}{8} & \multirow{2}{*}{$\mathrm{C}_{1} \leq 10 ; \mathrm{C}_{6} \leq 75 ; \mathrm{C}_{7} \leq 13$} & user criteria & DBCAFE & DBCAFE & \multirow{2}{*}{ DBCAFE } \\
\hline & & all criteria & BCDAFE & BCDAFE & \\
\hline
\end{tabular}



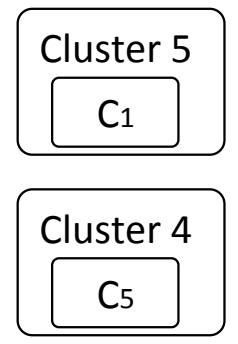

(a) Requirements network.

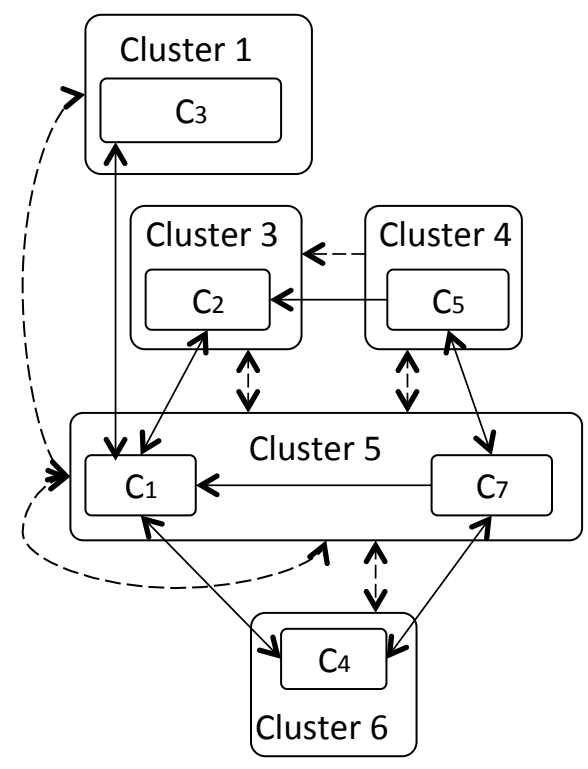

(b) Partial network.

Figure 7.8: ANP network for the first quality requirements example obtained using the Requirements network and the Partial network extraction methods.

alternatives that satisfy more user quality requirements; this can be directly observed in Table 7.5. However, some exceptions can be expected, as for example in the cases where one alternative is much better than all the other alternatives with respect to one or few criteria (e.g., it is the only alternative that satisfies one of the requirements), but which does not satisfy all quality requirements. This is the case of the fifth example in Table 7.5 (with $C_{1}, C_{3}$, and $C_{5}$ as user quality requirements), in which alternative $F$ is ranked first even if alternative $A$ satisfies more quality requirements. In this case, alternative $F$ is the only alternative that satisfies the requirement with respect to the criterion $C_{5}$; in the decision process, this alternative gets much more importance than the others with respect to this criterion, which is why it is ranked as the best one. However, this does not happen in all the cases, as can be seen from 
the eighth example (with $C_{1}, C_{3}$, and $C_{7}$ as user quality requirements) in which alternative $C$, which satisfies one requirement and is the only one that satisfies the requirement with respect to the criterion $C_{1}$, is ranked after alternatives $D$ and $B$, which satisfy two requirements.

\subsubsection{Discussion on the Model Extraction Methods Evalua- tion}

In the analysis of the results, alternative rankings obtained with the different network extraction methods are compared in order to determine which network extraction is the most suitable for producing recommendations in terms of user quality requirements. Such analysis leads to several conclusions.

In the case when alternatives are compared only with respect to the criteria that appear in user quality requirements, rankings of the extraction methods that produce complete and partial networks are identical in a large majority of the cases. This is because the part of the network related to alternatives is the same in both cases, which results in the same comparisons and entries in the corresponding part of the supermatrix.

In some cases, when alternatives are compared with respect to all the criteria in the network, the rankings of the extraction methods that produce complete and partial networks are also identical. This is because the selection of certain quality requirements can result in an identical or very similar networks in both extraction methods.

In some cases, alternatives that satisfy the highest number of user quality requirements are better ranked when alternatives are compared with respect to all the criteria in the network than when alternatives are compared only with respect to the criteria that appear in user quality requirements, which can be seen in the seventh example with the Partial network. This is because the importance of the criteria in these two cases are different, which leads to different rankings.

In the case when a decision maker is strictly interested in his or her quality requirements, the network that consists only of those quality requirements is the most suitable extraction method to be applied, because in most of the cases it is able to rank best those alternatives that satisfy more quality requirements, as shown in Table 7.5. In other cases, the extraction method that produces a partial network can be applied because it is computationally more efficient than the extraction method that produces a complete network.

Finally, with respect to the hypothesis formulated, the following conclusions can be drawn:

- H5. Those alternatives that satisfy a higher number of specified user quality requirements are ranked better in an extracted model which only contains those 
criteria appearing in specified user quality requirements.

It can be stated that using ANP networks that only contain quality criteria appearing in user quality requirements does not always ensure ranking better alternatives that satisfy a higher number of quality requirements. Even if this is an expected behavior for most cases, as it was previously discussed, if an alternative has the best result with respect to one or several criteria, it can be ranked better, regardless the number of satisfying quality requirements. Therefore, the hypothesis is false.

\subsection{Evaluation of the SemQuaRE Quality Model}

Chapter 5 presented SemQuaRE, a quality model for semantic technologies, and this section presents the evaluation of such quality model.

As there are no exact criteria to be referred to when evaluating quality models, the evaluation of SemQuaRE is based on similar evaluations found in the literature [8] and on criteria that are seen as being the most important for the semantic technology domain. Therefore, SemQuaRE has been evaluated along two aspects: representativeness and flexibility. The goal of this evaluation is to determine whether SemQuaRE is representative with respect to the current practice in semantic technology evaluation, and whether it can be easily extended with new tool types and quality characteristics.

The following hypotheses are made for this evaluation (Section 3.3):

- H6. The SemQuaRE quality model for semantic technologies is representative, which means that it covers the majority of the quality characteristics that are evaluated in practice.

- H7. The SemQuaRE quality model for semantic technologies is flexible, which means that it can be extended with new quality characteristics or new types of tools.

\subsubsection{Representativeness}

The purpose of the representativeness evaluation is to observe the inclusion of all the quality characteristics in the area of study [8]. This evaluation has been performed because SemQuaRE was initially built by taking into account only the evaluation results obtained in the SEALS project. Therefore, the goal of this evaluation is twofold: first, to determine whether the quality model proposed describes those quality characteristics and indicators that are evaluated in practice when assessing the quality of semantic technologies and, second, to identify whether there are any 
quality characteristics evaluated that are not included in SemQuaRE (which could be used to extend the quality model proposed).

In this evaluation, SemQuaRE is compared to the findings of the literature review presented in Section 2.3.1. From the literature review it can be observed that almost all the measures described in the publications conform to the quality characteristics that SemQuaRE describes, and only three domain-specific quality characteristics are not described in SemQuaRE: time behavior of semantic web services (found in five publications), resource utilization of ontology matching tools (found in one publication), and resource utilization of reasoning systems (found in two publications). Besides these, only one general quality characteristic found in the literature review is not described in SemQuaRE model, which is learnability.

\subsubsection{Flexibility}

In the semantic technology domain, due to the existence of tools that share functionalities of different types, it is important to have a unique quality model for all types of semantic technologies. As currently SemQuaRE does not include every type of technology nor every quality characteristic for the technology types it covers, the purpose of this evaluation is to observe how flexible the quality model proposed is in terms of its extension with new types of semantic technologies and with new domain-specific characteristics.

As mentioned in the previous section, some measures found in the literature review were not observed by the initial version of SemQuaRE; therefore, new domain-specific quality sub-characteristics have been defined for them. These are:

- Semantic web service time behavior. Degree to which the response, processing times and throughput rates of a product or system meet requirements when performing a semantic web service discovery task.

- Ontology alignment resource utilization. Degree to which the amount and types of resources used by a product or system meet the requirements when performing an ontology alignment task.

- Reasoning resource utilization. Degree to which the amount and types of resources used by a product or system meet the requirements when performing a reasoning task.

These sub-characteristics have been completely included into SemQuaRE together with their related set of quality measures and their formulas.

In order to further evaluate the flexibility of SemQuaRE, the evaluations of a new type of semantic technology, ontology annotation tools, that was not initially included in SemQuaRE have been analysed. The goal was to determine whether 
SemQuaRE is flexible enough so that evaluation results for other types of semantic technologies could be included.

The analysis of the ontology annotation tool evaluations included the publications of the proceedings of the International Semantic Web Conference and of the European/Extended Semantic Web Conference, which in total included five publications related to ontology annotation tool evaluation. In four publications the authors evaluate precision, three publications report results for recall, one publication provides results for normalized discounted cumulative gain and F-measure, and one publication provides results for correctness. All measures found are used to measure accuracy and, therefore, a new sub-characteristic, namely Ontology annotation accuracy, has been introduced into SemQuaRE. This sub-characteristic has been aligned with SQuaRE's Functional correctness quality sub-characteristic.

Table 7.6 shows the quality sub-characteristics and quality measures added to SemQuaRE during the flexibility evaluation. After this evaluation, one new type of semantic technology (i.e., ontology annotation tools) has been added to the model, with one quality sub-characteristic, four indicators, four derived measures, and one base measure. Furthermore, with respect to the semantic technologies that were already described in SemQuaRE, three new quality characteristics have been added, together with seven indicators and seven base measures.

Figure 7.9 shows the product quality characteristics and sub-characteristics (Section 2.2.4) in SemQuaRE after the flexibility evaluation. The quality in use part was not affected.

\subsubsection{Discussion on the SemQuaRE Quality Model Evalua- tion}

The evaluation of the SemQuaRE quality model consisted in comparing the semantic technology types, quality characteristics, and quality measures with the current practice in semantic technology evaluation, as well as in extending SemQuaRE with new tool types and characteristics. From the results of this evaluation, several conclusions can be outlined.

The analysis of the literature review presented in Section 2.3.1 and of SemQuaRE shows that the quality model proposed is quite representative with respect to the current practice in semantic technology evaluation and to the types of semantic technologies that it describes. Furthermore, a similar conclusion can be outlined with respect to the quality measures and quality characteristics.

One important aspect of the representativeness evaluation of SemQuaRE would consist in comparing SemQuaRE to other semantic technology quality models. However, to the best of the knowledge of the author of this thesis, SemQuaRE is the only example of a quality model for semantic technologies. 


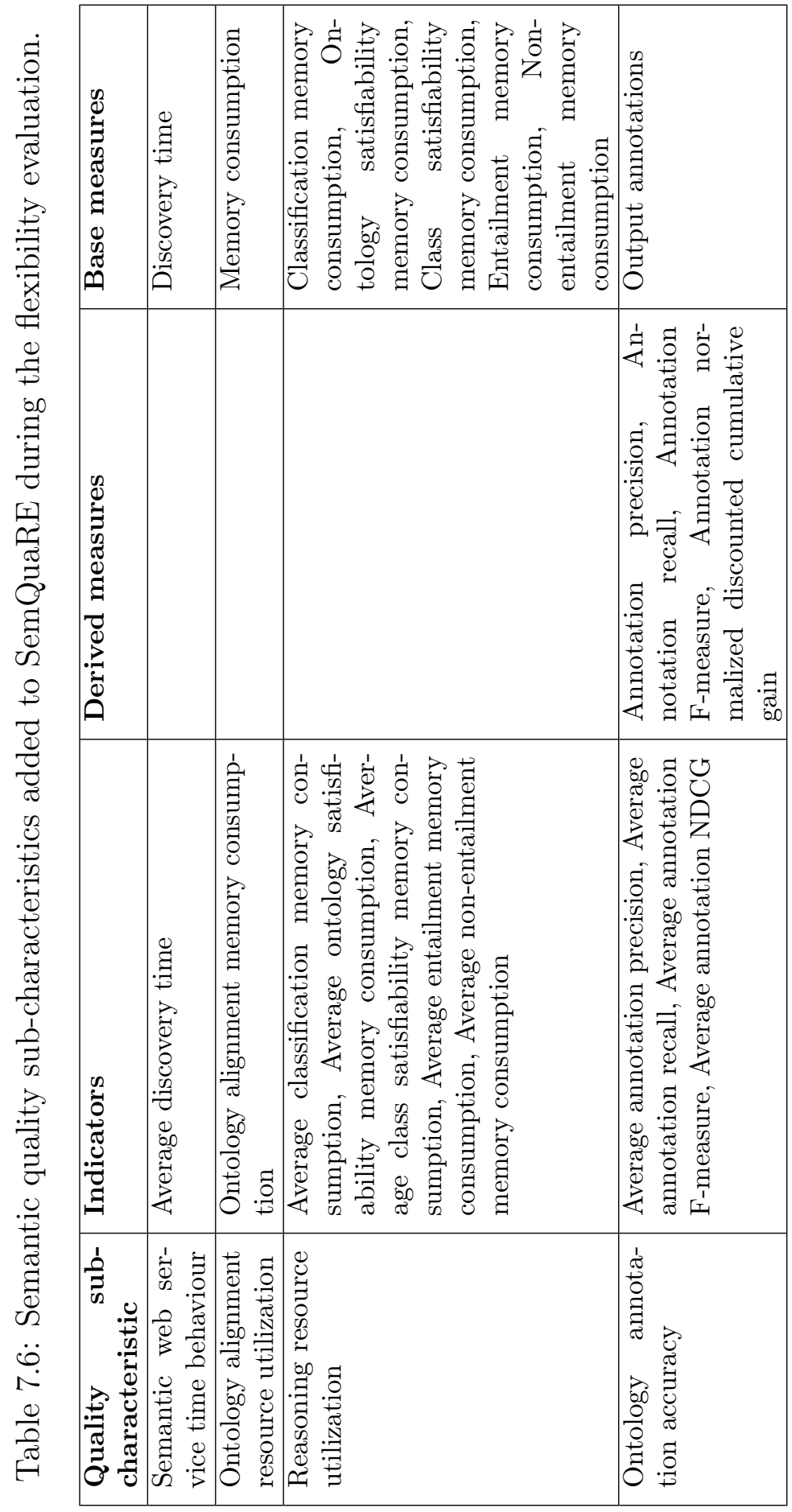




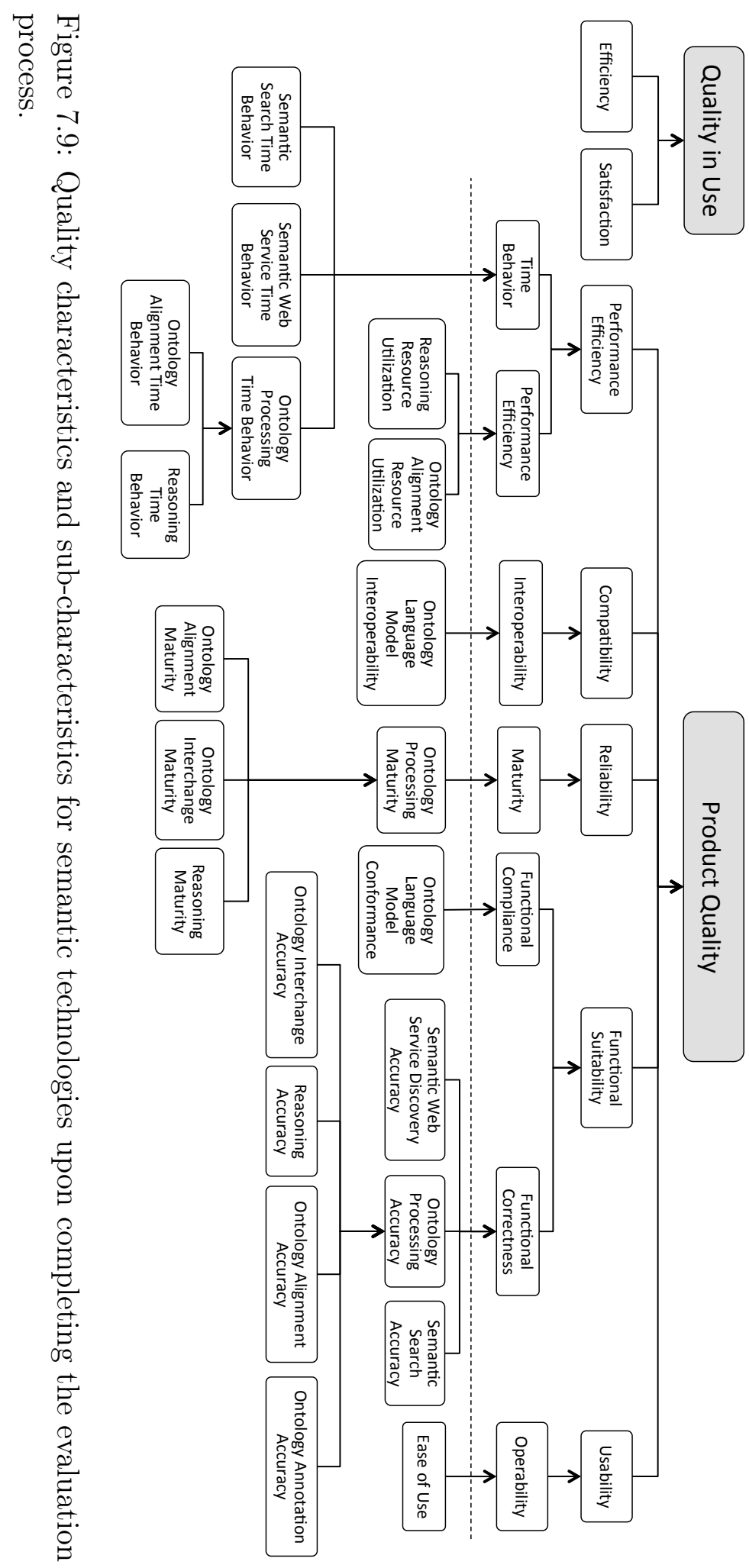


In the flexibility evaluation three new quality characteristics for the already described tool types were added to SemQuaRE, including the complete hierarchy of quality measures and related formulas. Furthermore, one new tool type with its corresponding quality characteristics, quality measures and formulas has been added to SemQuaRE in this evaluation. The inclusion of mentioned characteristics and tool types shows that the quality model can be easily extended.

With respect to the hypotheses stated in this section, the following conclusions can be outlined:

- H6. The SemQuaRE quality model for semantic technologies is representative. Since a large majority of the quality characteristics described in SemQuaRE is the one that is usually evaluated in the literature, it can be stated that SemQuaRE is representative with respect to the current practice. Therefore, this hypothesis is true.

- H7. The SemQuaRE quality model for semantic technologies is flexible.

The SemQuaRE quality model has been extended to include new tool types and new quality characteristics. Therefore, this hypothesis is true.

\subsection{Evaluation of the RIDER Framework with User Feedback}

Chapter 6 presented an instantiation of the RIDER framework in the semantic technology domain and one particular type of technology included in this instantiation, which is ontology matching tools. Since user quality requirements are an important part of the RIDER framework, feedback from users on RIDER recommendations is necessary in order to further evaluate the framework. Therefore, an evaluation relying on user feedback about recommendations in the ontology matching domain has been performed, which addresses the usefulness ${ }^{3}$ and effectiveness ${ }^{4}$ of RIDER according to SQuaRE. The ontology matching domain has been chosen for this evaluation in order to provide to users a simple scenario of use that will also serve for the purpose of evaluation (see evaluation plan 8, Section 3.4).

The goal of this evaluation is to examine user feedback about RIDER recommendations and to investigate whether users find the RIDER framework useful and effective in practice.

\footnotetext{
${ }^{3}$ Degree to which users are satisfied with their perceived achievement of pragmatic goals, including the results of use and the consequences of use.

${ }^{4}$ Accuracy and completeness with which users achieve specified goals.
} 
This evaluation covers the RIDER framework as a whole (Figure 7.10) in the domain of ontology matching tools, with the user quality requirements, ANP network, evaluation results and pairwise comparisons as inputs, and the rankings of alternatives that are obtained as outputs.

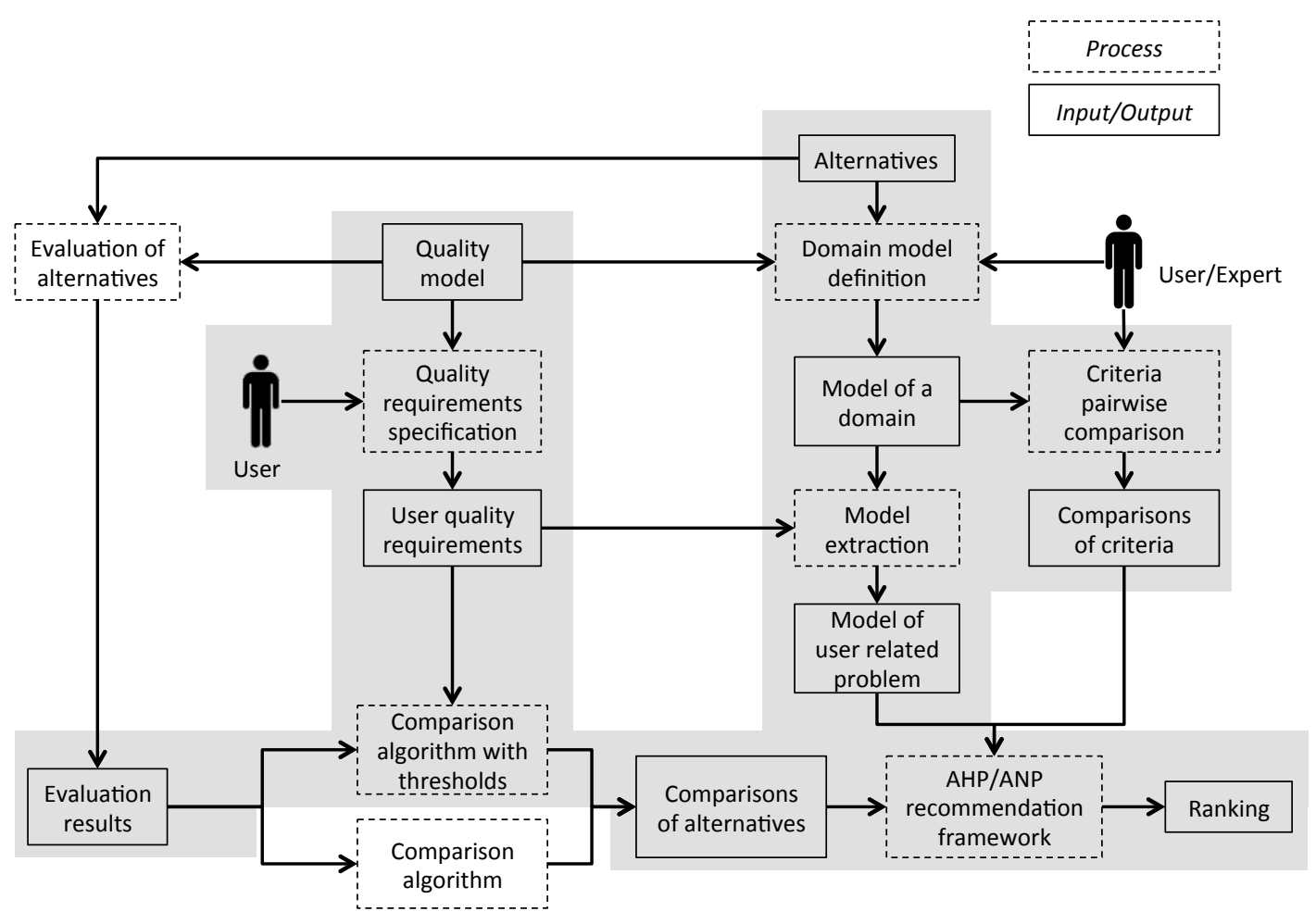

Figure 7.10: Evaluation of RIDER framework with user feedback.

The following hypotheses are made for this evaluation (Section 3.3):

- H8. The RIDER framework in the ontology matching domain produces recommendations that are accurate in the context of users' needs.

- H9. The RIDER framework in the ontology matching domain produces recommendations that satisfy users.

The evaluation of the usefulness and effectiveness of the RIDER framework has been performed by conducting individual interviews with users with an approximate duration of one hour per user. The following procedure has been applied during these interviews:

1. First, the user is presented with a set of tools, the ANP network, a set of evaluation results, and a set of use cases in which different quality requirements are taken into account. 
2. After the user is familiar with the previous inputs, he or she is asked, for each use case, to rank the tools from best to worst by taking into account the evaluation results and quality requirements related to the specific use case.

3. For each use case, the ranking provided by the user is compared to the ranking provided by the RIDER framework. In those cases when the two rankings are different, the user is presented with an explanation of the ranking provided by the RIDER framework. After this, the user is allowed to change his or her initial ranking.

4. Finally, after all the use cases have been examined, the user is asked to express his or her satisfaction with the RIDER rankings with a single note on a 5-point Likert scale [81], which is commonly used to measure user satisfaction, and where note 5 denotes the highest satisfaction.

The evaluation with user feedback allows to extract several parameters that can help in the evaluation of the hypotheses:

- The percentage of cases in which the initial user ranking agrees with the RIDER ranking and the percentage of cases in which the ranking after a user was allowed to change his or her initial ranking agrees with the RIDER ranking help in evaluating $H 8$. Furthermore, an additional parameter for evaluating $H 8$ includes the agreement of the user selection of the best tool with the RIDER recommendation of the best tool.

- The satisfaction note allows determining user satisfaction with the RIDER framework, and thus helps in evaluating $H 9$.

\subsubsection{Setup of the Evaluation with User Feedback}

The inputs in this evaluation, i.e., the ANP network of ontology matching tools, pairwise comparisons and evaluation results, are those that are described in Chapter 6 , with the difference that only a subset of the original evaluation results has been used in order to meet the interview time constraint. Furthermore, eight use cases to be used in this evaluation have been defined, where each use case is characterised by a different set of quality requirements.

The evaluation results to be used in this evaluation have been selected in such a way that, for each of the six characteristics for which the results are compiled, the best and the worst results are taken into account. This selection resulted in five different tools out of the initial nineteen, which have been assigned different names in the evaluation in order to avoid biases in those cases where an interviewed user is familiar with ontology matching tools. 
Table 7.7 shows the results for the five tools and the six quality characteristics used in this evaluation and Table 7.8 shows the eight use cases used in the evaluation. For each use case, a set of quality requirements is shown, together with the rankings obtained by the RIDER framework by using the Simple algorithm (Section 4.4) and Requirements network (Section 4.5). The importance of each quality requirement is shown in brackets; in those cases where all quality requirements are of equal importance, their importances are omitted.

Table 7.7: Evaluation results used in the evaluation with user feedback.

\begin{tabular}{|c|c|c|c|c|c|}
\cline { 2 - 6 } \multicolumn{1}{c|}{} & \multicolumn{5}{c|}{ Tools } \\
\hline Characteristics & A & B & C & D & E \\
\hline Precision & 0.89 & 0.98 & 0.99 & 0.14 & 0.25 \\
\hline Recall & 0.5 & 0.68 & 0.64 & 0.1 & 0.08 \\
\hline F-measure & 0.64 & 0.8 & 0.77 & 0.11 & 0.12 \\
\hline H-measure & 0.64 & 0.8 & 0.77 & 0.11 & 0.12 \\
\hline Time (s) & 13.5 & 66670 & 1750 & 16504 & 554 \\
\hline Errors (\%) & 0 & 0 & 25 & 0 & 25 \\
\hline
\end{tabular}

\subsubsection{Results of the Evaluation with User Feedback}

In total, 30 users have been interviewed. Table 7.9 presents the results for each of the eight use cases showing user rankings and the number of users that defined each ranking. The initial user rankings are separated from the user rankings obtained after having the possibility to change the initial ranking (final ranking).

It can be observed that, although the agreement of user initial rankings with the RIDER rankings is not very high, the user selection of the best tools agrees with the

Table 7.8: Quality requirements and RIDER rankings for each use case.

\begin{tabular}{|c|c|c|}
\hline $\begin{array}{c}\text { Use } \\
\text { case }\end{array}$ & Quality requirements & Ranking \\
\hline 1 & F-measure $>0.75$ & BCAED \\
\hline 2 & Errors $<20$ & ADBCE \\
\hline 3 & Precision $>0.8 ;$ Time $<20$ & ACBED \\
\hline 4 & Recall $>0.6 ;$ Errors $<20$ & BACDE \\
\hline 5 & Time $<20(0.4) ;$ Errors $<10(0.4) ;$ F-measure $>0.75(0.2)$ & ABCDE \\
\hline 6 & Errors $<5(0.5) ;$ Precision $>0.8(0.35) ;$ Recall $>0.6(0.15)$ & BACDE \\
\hline 7 & Precision $>0.95(0.65) ;$ H-measure $>0.75(0.35)$ & CBAED \\
\hline 8 & Time $<20(0.45) ;$ Precision $>0.95(0.35) ;$ H-measure $>0.75(0.2)$ & ACBED \\
\hline
\end{tabular}


Table 7.9: Rankings obtained by RIDER and by the users.

\begin{tabular}{|c|c|c|c|c|c|}
\hline Use case & RIDER & \multicolumn{2}{|c|}{ Initial ranking } & \multicolumn{2}{|c|}{ Final ranking } \\
\hline \multirow{4}{*}{1} & \multirow{4}{*}{ BCAED } & 24 & BCAED & 28 & BCAED \\
\hline & & 4 & BCADE & 2 & BCADE \\
\hline & & 1 & CBAED & 0 & CBAED \\
\hline & & 1 & CAEDB & 0 & CAEDB \\
\hline \multirow{4}{*}{2} & \multirow{4}{*}{ ADBCE } & 0 & ADBCE & 11 & ADBCE \\
\hline & & 15 & BADCE & 10 & BADCE \\
\hline & & 14 & ABDCE & 9 & ABDCE \\
\hline & & 1 & ADBEC & 0 & ADBEC \\
\hline \multirow{5}{*}{3} & \multirow{5}{*}{ ACBED } & 25 & ACBED & 29 & ACBED \\
\hline & & 2 & ACEBD & 1 & ACEBD \\
\hline & & 1 & ABCED & 0 & ABCED \\
\hline & & 1 & ACDBE & 0 & ACDBE \\
\hline & & 1 & ACEDB & 0 & ACEDB \\
\hline \multirow{3}{*}{4} & \multirow{3}{*}{ BACDE } & 19 & BACDE & 26 & BACDE \\
\hline & & 8 & BCADE & 3 & BCADE \\
\hline & & 3 & BADCE & 1 & BADCE \\
\hline \multirow{12}{*}{5} & \multirow{12}{*}{$\mathrm{ABCDE}$} & 1 & ABCDE & 21 & ABCDE \\
\hline & & 16 & ABDCE & 6 & ABDCE \\
\hline & & 1 & ADCBE & 1 & ADCBE \\
\hline & & 1 & ACBDE & 1 & ACBDE \\
\hline & & 1 & ACEBD & 1 & ACEBD \\
\hline & & 3 & ADBCE & 0 & ADBCE \\
\hline & & 2 & ABCED & 0 & ABCED \\
\hline & & 1 & ADCEB & 0 & ADCEB \\
\hline & & 1 & AECDB & 0 & AECDB \\
\hline & & 1 & ACDBE & 0 & ACDBE \\
\hline & & 1 & ADBEC & 0 & ADBEC \\
\hline & & 1 & CABDE & 0 & CABDE \\
\hline \multirow{5}{*}{6} & \multirow{5}{*}{ BACDE } & 18 & BACDE & 27 & BACDE \\
\hline & & 8 & BADCE & 2 & BADCE \\
\hline & & 1 & CBADE & 1 & CBADE \\
\hline & & 2 & BCADE & 0 & BCADE \\
\hline & & 1 & ABCDE & 0 & ABCDE \\
\hline \multirow{4}{*}{7} & \multirow{4}{*}{ CBAED } & 17 & CBAED & 22 & CBAED \\
\hline & & 11 & BCAED & 6 & BCAED \\
\hline & & 1 & CBADE & 1 & CBADE \\
\hline & & 1 & BCADE & 1 & BCADE \\
\hline \multirow{7}{*}{8} & \multirow{7}{*}{ ACBED } & 11 & ACBED & 23 & ACBED \\
\hline & & 11 & CBAED & 5 & CBAED \\
\hline & & 3 & ABCED & 1 & ABCED \\
\hline & & 1 & BCAED & 1 & BCAED \\
\hline & & 2 & AECDB & 0 & AECDB \\
\hline & & 1 & ACEBD & 0 & ACEBD \\
\hline & & 1 & ACBDE & 0 & ACBDE \\
\hline
\end{tabular}


RIDER one in most of the use cases. This is especially the case in the first five use cases where almost all users selected as the best tools those tools that are selected as the best in RIDER.

When it comes to the user final rankings, the agreement of the user rankings with the RIDER rankings is significantly higher than that in the initial rankings. Also, the user final selection of the best tools is slightly higher than the initial selection.

The average satisfaction note of the 30 users in this evaluation is 4.45 , which implies a high satisfaction of users with the RIDER rankings. To a certain extent, the high satisfaction can be expected having in mind the results of the evaluation and the high agreement of the user final rankings with the RIDER rankings, which can be directly observed in Table 7.9.

\subsubsection{Discussion on the Evaluation with User Feedback}

The evaluation of the RIDER framework with user feedback consisted in interviewing 30 users about 8 use cases, which resulted in 240 different cases. From the analysis of these cases, several conclusions can be outlined.

It has been observed that the initial user ranking did not agree with the RIDER ranking in all the cases. This is because:

a) The users have made errors when processing the evaluation results, which happened in $15 \%$ of the cases with different rankings. For example, in use case 5 one user changed the ranking of alternatives $C$ and $D$ because he overlooked the results for all three criteria in user quality requirements. These errors were corrected in the final ranking.

b) The users had different criteria priorities because of their personal preferences, which happened in $45 \%$ of the cases with different rankings. For example, in use case 6 one user provided a different ranking because she took into account the time criterion more than other criteria (even if such criterion did not appear in the quality requirements).

With the exception of those cases where users had different preferences that were not fed into the system, RIDER accuracy can be considered as high, since the user rankings agreed with the RIDER rankings in $78 \%$ of the cases. Furthermore, if focused on the selection of the best tools, the user selections agreed with the RIDER ones in $90 \%$ of the cases. This means that the differences in the user rankings in the large majority of the cases are due to disagreement in the ranking of the tools in the middle or in the end of the ranking list.

By observing the number of cases in which the user has made an error and the number of quality requirements in these cases, it can be concluded that the number of quality requirements does not influence the user errors. 
In the majority of the cases, the difference between user rankings and RIDER rankings is due to the difference in the ranking of only two tools. For example, in use case 4 some users ranked tool $C$ to be better than tool $A$, while RIDER ranked tool $A$ to be better than tool $C$.

With respect to the hypotheses, the following conclusions an be outlined:

- H8. The RIDER framework in the ontology matching domain produces recommendations that are accurate in the context of users' needs.

Although the accuracy of the RIDER recommendations taking into account the initial user recommendations is not very high, this accuracy becomes significantly higher after user errors are taken into account. Furthermore, the accuracy in terms of the best tools is high in both the initial rankings and in the rankings after taking into account user errors. Therefore, this hypothesis is true.

- H9. The RIDER framework in the ontology matching domain produces recommendations that satisfy users.

Since the average satisfaction of users with the RIDER rankings can be considered as high (4.45 of 5), this hypothesis is true. 



\section{Chapter 8}

\section{Conclusions and Future Work}

As stated in Chapter 3, one of the objectives of this thesis is to advance the current state of the art in the Multiple Criteria Decision Making field by providing a Multiple Criteria Decision Making framework that extends the Analytic Network Process and that takes advantage of evaluation results of alternatives and of user quality requirements over such alternatives. Another objective is to apply this MCDM framework in the Semantic Web field for semantic technology recommendation.

The objectives of this thesis are fulfilled by the main contributions of the thesis:

- Domain-independent algorithms based on Saaty's pairwise comparison scale, for the automatic comparison of alternatives according to alternatives' evaluation results.

The algorithms for the automatic comparison of alternatives are the core of the RIDER framework, which is designed to rely on evaluation results instead of on expert or user opinions. These algorithms are domain-independent and can also be easily applied for any comparison that is based on Saaty's pairwise comparison scale. When evaluation results are available, the algorithms provide the possibility for the automatic comparison of alternatives and support the inclusion of new results and alternatives without the need for expert comparisons.

Since several comparison algorithms have been developed, different algorithms can be applied in different cases, depending on the availability of evaluation results and on the specification of user quality requirements. This means that comparison algorithms cover a broad range of use cases, leaving decision makers several possibilities for exploitation of these algorithms.

The algorithms for the automatic comparison of alternatives are designed to produce outputs on the Saaty's scale, i.e., to produce an output which is a 
natural number between 1 and 9 . When it comes to the number of outputs of a comparison algorithm and to the distance between the maximum and the minimum output, as shown in the evaluation these depend on the distribution and on the range of evaluation results (H1, Section 7.1). Furthermore, as shown in the same evaluation, the quality of the comparison algorithm, as defined in Section 7.1, improves when all evaluation results are available (H2, Section 7.1). Additionally, since the comparison algorithms are also designed to take into account user quality requirements, the evaluation has shown that these quality requirements influence the comparisons performed by the algorithm and that they help in discriminating between results ( $H_{3}$ and $H_{4}$, Section 7.2).

- A set of methods for the dynamic construction of the AHP and ANP models that are based on user quality requirements.

The methods for the dynamic construction of the ANP and AHP models help to tie the recommendation process to user quality requirements. By starting from the complete model of any particular field, these methods exploit user quality requirements for the automatic adaptation of the model to these quality requirements leading to recommendations that satisfy the higher number of them, as shown in the evaluation process (H5, Section 7.3). Such methods also help in reducing the computational complexity of a decision problem in the cases of large and complex fields.

Model extraction methods, together with the comparison algorithms, enable the automatisation of the recommendation process with the ANP or AHP. In RIDER, experts or users are only needed for the construction of the initial model of a particular domain, while the construction of the model that is suitable for specific user quality requirements, as well as the comparison of alternatives, can be performed automatically. This means that less effort from users or experts is required, hence making the whole recommendation process less expensive.

- Software that supports the proposed MCDM framework.

RIDER has been implemented in a generic Java API and in a generic web application. The web application can be instantiated in any field, giving experts in a particular field a valuable asset for delivering recommendations to their clients, as well as bringing benefits to users who can directly use the instantiated web application.

- SemQuaRE, the quality model for semantic technologies, which extends the ISO SQuaRE software quality model.

SemQuaRE, a quality model for the evaluation of semantic technologies developed in this thesis as an artefact in the instantiation of RIDER in the semantic 
technology field, is independent from the instantiation and can be a valuable asset that provides a framework for the evaluation and comparison of semantic technologies. SemQuaRE is particularly significant for the community in several aspects:

- It provides a consistent terminology for semantic technology quality, even if it is limited to its current scope.

- Within such scope, it gives comprehensive definitions of all its elements, i.e., quality characteristics and measures.

- Although some problems with the ISO quality models have been identified (as discussed in Section 2.2.6), SemQuaRE has introduced quality measures specific to semantic technologies and it has also specified formulas for all derived measures and indicators, which result in reducing ambiguities in the model and providing guidance of how to evaluate and measure the quality of semantic technologies.

- It serves developers of new semantic tools as a checklist for semantic technology quality requirements.

Furthermore, it can be noted that SemQuaRE is representative and extensible, and that new quality measures or characteristics can be introduced and categorized, as shown during the evaluation process ( $H 6$ and $H^{\gamma}$, Section 7.4). Also, new types of semantic technologies can be included into the model, as presented for the case of ontology annotation tools.

- The application of the RIDER framework in the semantic technology field, including a web application for the recommendation of semantic technologies that takes into account users quality requirements and existing semantic technology evaluation results.

Relying on SemQuaRE and in agreement with expert opinions, the ANP network for semantic technologies has been developed, and the necessary pairwise comparisons have been performed. The experience in developing the ANP network for semantic technologies shows that this task is easier to perform when a reference such as a quality model exists.

The semantic technology recommendation use case has been implemented using the RIDER generic web application and it allows users to specify their quality requirements and to get recommendations of the semantic technologies that better suit their needs. The web application supports Semantic Web developers in choosing the semantic technologies that are the best for solving their problems.

The semantic technology use case suggests that RIDER can be successfully applied in any field. Furthermore, the web application that is available can be 
a valuable resource for experts who can use it and provide users with a tool for recommendations without the knowledge in the MCDM field, and also for users that get fast recommendations according to their quality requirements.

Even though this thesis presents advances to the fields of Multiple Criteria Decision Making and Semantic Web technologies, there are still some open problems that have not been addressed in this thesis, or that have come out as a consequence of the advances proposed in it. These problems encompass the lines of future work:

- This thesis has provided a set of comparison algorithms and it can be expected that better comparison algorithms appear in the future; therefore, this thesis has also provided an evaluation framework to assess their quality. One line of future work consists in defining new comparison algorithms and comparing them to the existing ones, as well as defining better quality metrics for assessing comparison algorithms.

- One of the advantages of the Saaty's pairwise comparison method is that it allows the calculation of the consistency of pairwise comparisons. The comparison algorithms developed in this thesis enable the automatic comparison of alternatives based on their evaluation results and user quality requirements; however, these algorithms have not been evaluated in terms of the consistency of their comparisons and, therefore, one line of future work includes an additional evaluation of the comparison algorithms in the context of pairwise comparison consistency.

- The evaluation of the comparison algorithms only takes into account the interval scale. However, as the ratio and interval scales behave identically for most statistical analyses, the conclusions obtained for the interval scale can be applied to ratio scale as well. Future work includes performing evaluations that take into account other types of scales.

- With respect to the RIDER framework, one potential line of work consists in applying it and providing recommendations in other fields (e.g., ontologies, Linked Data). This will help in further validating the applicability of RIDER framework.

- SemQuaRE has been built using the bottom-up methodology described in Section 5.1. Although the author of this thesis has not found any bottom-up approach for extending quality models in the literature, some authors suggest that a bottom-up approach is important when building quality models [29]. The bottom-up approach that was used is not validated, so one line of future work consists of performing an evaluation and providing details about the validation and usefulness of such approach. 
- The SemQuaRE evaluation process relied only on a literature review, i.e., limited venues that include the most relevant conferences and workshops in the semantic field, and limited tool types. Although some venues were implicitly included in the study (some editions of the Ontology Alignment Evaluation Initiative $^{1}$ and the Semantic Web Service Challenge ${ }^{2}$ have been addressed in the SEALS project and in the IWEST workshop series), in order to get a more complete quality model the future work will consist of extending the analysis to other types of tools and to other evaluation campaigns (e.g., the Semantic Web Challenge ${ }^{3}$, the Triplification Challenge ${ }^{4}$, or the Linked Data $\mathrm{Cup}^{5}$ ), as well as on relevant journals.

- SemQuaRE is a hierarchical quality model, as those of the ISO 9126 and the SQuaRE standards are, and this is regarded as an important factor for clear and unambiguous quality models [8]. Furthermore, Bertoa et al. [10] argue that the structure and organization of the model influence its understandability and, in this sense, it can be assumed that SemQuaRE provides a high degree of understandability to its users; however, the evaluation of understandability of SemQuaRE is planned for future work.

- Although SemQuaRE is quite complete, it is reasonable to expect some advances in the future, most notably in including additional types of semantic technologies. Therefore, one line of future work consists of extending the SemQuaRE, as well as extending the ANP network for semantic technologies and, consequently, the coverage of the semantic technology recommendation system.

- The ANP network for semantic technologies consists of a number of dependent criteria, and in every particular use case (i.e., for every set of user quality requirements) it is used in the network extraction process (Section 4.5) for extracting a subnetwork related to a use case; in this way, dependencies in the model are preserved. One alternative solution is to apply multiattribute analysis in order to reduce the complexity of the ANP network for semantic technologies, and possibly to obtain a model with no dependencies between the criteria (i.e., a hierarchy). Such approach is left to be investigated in future work, as well as a comparison of the two mentioned approaches.

- More advanced scenarios in the RIDER web application are planned for future work. For example, one scenario could provide feedback in the process of quality

\footnotetext{
${ }^{1}$ http://oaei.ontologymatching.org/

${ }^{2}$ http://sws-challenge.org/wiki/

${ }^{3}$ http://challenge.semanticweb.org/

${ }^{4}$ http://triplify.org/Challenge

${ }^{5}$ http://i-semantics.tugraz.at/i-challenge/call-for-submissions
} 
requirements specification, such that if a user chooses a quality requirement according to which all the tools are highly similar, a suggestion for omitting such quality requirement from the recommendation process can be given.

- Since some experienced users might be interested in providing their own inputs in the recommendation process, one line of future work consists of enabling the users of the RIDER web application the possibility to provide inputs in terms of modifications of the pairwise comparisons or model extractions, which are otherwise performed automatically.

- The results of the evaluation of the instantiation of the RIDER framework in the semantic technology domain rely on ontology matching tools; one line of future work consists of extending the evaluation by taking into account other tool types and other domains. 


\section{Bibliography}

[1] W. Abramowicz, R. Hofman, W. Suryn, and D. Zyskowski. SQuaRE based Web services quality model. In Proceedings of The International MultiConference of Engineers and Computer Scientists. Hong Kong, pages 827-835, 2008.

[2] N. Ahmad and P. A. Laplante. Software project management tools: Making a practical decision using AHP. In Proceedings of the 30th IEEE Software Engineering Workshop (SEW06). Columbia, Maryland, USA, pages 76-84, 2006.

[3] H. Al-Kilidar, K. Cox, and B. Kitchenham. The use and usefulness of the ISO/IEC 9126 quality standard. In Proceedings of the 2005 IEEE International Symposium on Empirical Software Engineering. Noosa Heads, Australia, pages 126-132, 2005.

[4] J. A. Alonso and M. T. Lamata. Consistency in the Analytic Hierarchy Process: A new approach. International Journal of Uncertainty, Fuzziness and Knowledge-Based Systems, 14(04):445-459, 2006.

[5] M. A. Azadeh and S. N. Shirkouhi. Evaluating simulation software using Fuzzy Analytical Hierarchy Process. In Proceedings of the 2009 Spring Simulation MultiConference. San Diego, California, 2009.

[6] M. Azuma. SQuaRE: The next generation of the ISO/IEC 9126 and 14598 international standards series on software product quality. In Proceedings of the European Software Control and Metrics Conference (ESCOM). London, UK, pages 337-346, 2001.

[7] F. Baader, M. Knechtel, and R. Peñaloza. A generic approach for large-scale ontological reasoning in the presence of access restrictions to the ontology's axioms. In Proceedings of the 8th International Semantic Web Conference (ISWC2009). Chantilly, Virginia, USA, pages 49-64. Springer-Verlag, 2009.

[8] B. Behkamal, M. Kahani, and K. A. Akbari. Customizing ISO 9126 quality model for evaluation of B2B applications. Information and Software Technology, 51(3):599-609, 2009. 
Bibliography

[9] T. Berners-Lee, J. Hendler, and O. Lassila. The Semantic Web. Scientific American, 284(5):34-43, 2001.

[10] M. F. Bertoa, J. M. Troya, and A. Vallecillo. Measuring the usability of software components. Journal of Systems and Software, 79(3):427-439, 2006.

[11] B. W. Boehm, J R. Brown, and M. Lipow. Quantitative evaluation of software quality. In Proceedings of the 2nd International Conference on Software Engineering. San Francisco, California, USA, pages 592-605, 1976.

[12] M. Bombardieri and F. A. Fontana. A specialisation of the SQuaRE quality model for the evaluation of the software evolution and maintenance activity. In Proceedings of the Automated Software Engineering Workshops (ASE2008). L'Aquila, Italy, pages 110-113, 2008.

[13] P. Botella, X. Burgués, J. P. Carvallo, X. Franch, J. A. Pastor, and C. Quer. Towards a quality model for the selection of ERP systems. Component-Based Software Quality, 2693:225-245, 2003.

[14] P. Botella, X. Burgués, J. P. Carvallo, X. Franch, G. Grau, J. Marco, and C. Quer. ISO/IEC 9126 in practice: What do we need to know? In Proceedings of the 1st Software Measurement European Forum. Rome, Italy, pages 297-306, 2004.

[15] J. P. Brans and B. Mareschal. PROMETHEE methods. In Multiple criteria decision analysis: State of the Art surveys, pages 163-186. Springer, 2005.

[16] J. P. Brans and P. Vincke. A preference ranking organization method. Management Science, 31(6):647-656, 1985.

[17] J. Brooke. Usability evaluation in industry, chapter SUS: A quick and dirty usability scale, pages 189-194. Taylor and Francis, 1996.

[18] L. Cabral, S. Tymaniuk, D. Winkler, and I. Toma. D14.3: Results of the first evaluation of Semantic Web service tools. Technical report, SEALS Consortium, November 2010.

[19] J. P. Carvallo, X. Franch, and C. Quer. Defining a quality model for mail servers. In Proceedings of the 2nd International Conference on COTS-based Software Systems (ICCBSS 2003). Ottawa, Canada, pages 51-61, 2003.

[20] J. P. Cavano and J. A. McCall. A framework for the measurement of software quality. ACM SIGMETRICS Performance Evaluation Review, 7(3-4):133-139, 1978. 
[21] A. N. Chitnis, A. Qasem, and J. Heflin. Benchmarking reasoners for multiontology applications. In Proceedings of the 5th International Workshop on Evaluation of Ontologies and Ontology-based Tools. Busan, Korea, pages 21-30, 2007.

[22] E. Colombo and C. Francalanci. Selecting CRM packages based on architectural, functional, and cost requirements: Empirical validation of a hierarchical ranking model. Requirements Engineering, 9(3):186-203, 2004.

[23] A. Damodaran. Strategic risk taking: A framework for risk management. Wharton School Publishing, 2007.

[24] K. Delhi Babu, P. Govindarajulu, A. Ramamohana Reddy, and A. N. Aruna Kumari. An integrated approach of AHP-GP and visualization for selection of software architecture: A framework. In Proceedings of the 2010 International Conference on Advances in Computer Engineering (ACE2010). Bangalore, Karnataka, India, pages 334-338, 2010.

[25] D. Diakoulaki, C. Henggeler Antunes, and A. Gomes Martins. MCDM and energy planning. In Multiple criteria decision analysis: State of the Art surveys, pages 859-897. Springer, 2005.

[26] V. A. Dimitrova. Application of the Analytic Network Process (ANP) in a framework of ERP systems implementation success. In Proceedings of the 4 th International IEEE Conference on Intelligent Systems. Varna, Bulgaria, volume 3, pages 2019-2024, 2008.

[27] F. J. Domínguez-Mayo, M. J. Escalona, M. Mejías, and A. H. Torres. A quality model in a quality evaluation framework for MDWE methodologies. In Proceedings of the 4th International Conference on Research Challenges in Information Science (RCIS2010). Nice, France, pages 495-506, 2010.

[28] F. J. Domínguez-Mayo, M. J. Escalona, M. Mejías, M. Ross, and G. Staples. Quality evaluation for model-driven web engineering methodologies. Information and Software Technology, 54(11):1265-1282, 2012.

[29] R. G. Dromey. Software product quality: theory, model, and practice. Technical report, Software Quality Institute, Brisbane, Australia, 1998.

[30] O. Durán. Computer-aided maintenance management systems selection based on a fuzzy AHP approach. Advances in Engineering Software, 42(10):821-829, 2011. 
[31] E. Dustin, J. Rashka, and D. McDiarmid. Quality web systems: performance, security, and usability. Addison-Wesley, 2002.

[32] J. Euzenat and P. Shvaiko. Ontology matching. Springer-Verlag, 2nd edition, 2013.

[33] J. Euzenat, A. Ferrara, W. R. van Hage, L. Hollink, C. Meilicke, A. Nikolov, D. Ritze, F. Scharffe, P. Shvaiko, H. Stuckenschmidt, O. Sváb-Zamazal, and C. Trojahn dos Santos. Results of the ontology alignment evaluation initiative 2011. In Proceedings of the 6th International Workshop on Ontology Matching. Bonn, Germany, 2011.

[34] J. Figueira, S. Greco, and M. Ehrgott. Multiple criteria decision analysis: State of the art surveys. Springer, 2005.

[35] J. Figueira, V. Mousseau, and B. Roy. ELECTRE methods. In Multiple criteria decision analysis: State of the art surveys, pages 133-153. Springer, 2005.

[36] X. Franch and J. P. Carvallo. Using quality models in software package selection. IEEE Software, 20(1):34-41, 2003.

[37] F. Garcia, M. F. Bertoa, C. Calero, A. Vallecillo, F. Ruiz, M. Piattini, and M. Genero. Towards a consistent terminology for software measurement. Information and Software Technology, 48(8):631-644, 2006.

[38] R. García-Castro. Benchmarking Semantic Web technology. Studies on the Semantic Web vol. 3. AKA Verlag - IOS Press, 2010.

[39] R. García-Castro and A. Gómez-Pérez. Interoperability results for Semantic Web technologies using OWL as the interchange language. Web Semantics: Science, Services and Agents on the World Wide Web, 8:278-291, 2010.

[40] R. García-Castro, A. Gómez-Pérez, O. Muñoz-García, and L Nixon. Towards a component-based framework for developing semantic web applications. In Proceedings of the 3rd Asian Semantic Web Conference (ASWC2008). Bangkok, Thailand, pages 197-211. Springer, 2008.

[41] R. García-Castro, M. Esteban-Gutiérrez, and A. Gómez-Pérez. Towards an infrastructure for the evaluation of semantic technologies. In Proceedings of the eChallenges Conference (e-2010). Warsaw, Poland, pages 1-8. IEEE, 2010.

[42] R. García-Castro, S. Grimm, I. Toma, M. Schneider, A. Marte, and S. Tymaniuk. D10.3: Results of the first evaluation of ontology engineering tools. Technical report, SEALS Consortium, November 2010. 
[43] R. García-Castro, S. Tymaniuk, and M. Schneider. D10.6: Results of the second evaluation of ontology engineering tools. Technical report, SEALS Consortium, June 2012.

[44] B. Glimm, I. Horrocks, B. Motik, and G. Stoilos. Optimising ontology classification. In Proceedings of the 9th International Semantic Web Conference (ISWC20010). Shanghai, China, pages 225-240. Springer-Verlag, 2010.

[45] M. Godse, R. Sonar, and S. Mulik. Web service selection based on Analytical Network Process approach. In Proceedings of the IEEE Asia-Pacific Services Computing Conference (APSCC08). Yilan, Taiwan, pages 1103-1108, 2008.

[46] A. Gómez-Pérez, M. Fernández-López, and O. Corcho. Ontological Engineering: With examples from the areas of Knowledge Management, e-Commerce and the Semantic Web. Springer-Verlag New York, 2004.

[47] J. Gonzáez-Pachón and C. Romero. A method for dealing with inconsistencies in pairwise comparisons. European Journal of Operational Research, 158(2): 351-361, 2004.

[48] J. González-Pachón and C. Romero. Inferring consensus weights from pairwise comparison matrices without suitable properties. Annals of Operations Research, 154(1):123-132, 2007.

[49] A. Guitouni and J. M. Martel. Tentative guidelines to help choosing an appropriate MCDA method. European Journal of Operational Research, 109 (2):501-521, 1998.

[50] Y. Guo, Z. Pan, and J. Heflin. LUBM: A benchmark for OWL knowledge base systems. Web Semantics: Science, Services and Agents on the World Wide Web, 3(2-3):158-182, 2005.

[51] P. T. Harker. Derivatives of the Perron root of a positive reciprocal matrix: With application to the Analytic Hierarchy Process. Applied Mathematics and Computation, 22(2):217-232, 1987.

[52] B. Hecht. Choose the right ERP software. Datamation, 43(3):56-61, 1997.

[53] C. Herssens, I. J. Jureta, and S. Faulkner. Dealing with quality tradeoffs during service selection. In Proceedings of the International Conference on Autonomic Computing (ICAC2008). Chicago, Illinois, USA, pages 77-86, 2008.

[54] V. Hlupic. Simulation software selection using SimSelect. Simulation, 69(4): 231-239, 1997. 
[55] W. Ho. Integrated Analytic Hierarchy Process and its applications - A literature review. European Journal of Operational Research, 186(1):211-228, 2008.

[56] H. Hou, J. Yang, Q. B. Song, and K. G. Hao. The research of BPM software trustworthy evaluation model. In Proceedings of the 1st International Workshop on Education Technology and Computer Science. Wuhan, Hubei, China, volume 3, pages 815-823, 2009.

[57] C. H. Hsu, C. M. Yang, T. S. Chen, and C. Y. Chen. Applying AHP method select online shopping platform. In Proceedings of the 7th International Conference on Service Systems and Service Management (ICSSSM). Tokyo, Japan, pages $1-5,2010$.

[58] IEEE. IEEE 829 - 2008, Standard for Software and System Test Documentation. Technical report, Institute of Electrical and Electronics Engineers, 2008.

[59] A. Ishizaka and M. Lusti. How to derive priorities in AHP: A comparative study. Central European Journal of Operations Research, 14(4):387-400, 2006.

[60] ISO. ISO/IEC 14598 series:1999, Software engineering - Product evaluation. Technical report, International Organization for Standardization, 1999.

[61] ISO. ISO/IEC 9126-1:2001, Software engineering - Product quality - Part 1: Quality model. Technical report, International Organization for Standardization, 2001.

[62] ISO. ISO/IEC 9126-2:2003, Software engineering - Product quality - Part 2: External metrics. Technical report, International Organization for Standardization, 2003.

[63] ISO. ISO/IEC 9126-3:2003, Software engineering - Product quality - Part 3: Internal metrics. Technical report, International Organization for Standardization, 2003.

[64] ISO. ISO/IEC 15939:2007, Systems and software engineering - Measurement process. Technical report, International Organization for Standardization, 2007.

[65] ISO. ISO/IEC 25010:2011, Systems and software engineering - Systems and software Quality Requirements and Evaluation (SQuaRE) - System and software quality models. Technical report, International Organization for Standardization, 2011.

[66] A. S. Jadhav and R. M. Sonar. Evaluating and selecting software packages: A review. Information and Software Technology, 51(3):555-563, 2009. 
[67] A. S. Jadhav and R. M. Sonar. Analytic Hierarchy Process (AHP), Weighted Scoring Method (WSM), and Hybrid Knowledge Based System (HKBS) for software selection: A comparative study. In Prooceedings of the 2nd International Conference on Emerging Trends in Engineering and Technology (ICETET). Washington DC, USA, pages 991-997, 2009.

[68] H. W. Jung and B. Choi. Optimization models for quality and cost of modular software systems. European Journal of Operational Research, 112(3):613-619, 1999.

[69] R. L. Keeney and H. Raiffa. Decisions with multiple objectives: Preferences and value tradeoffs. Cambridge University Press, 1993.

[70] W. De Keyser and P. Peeters. A note on the use of PROMETHEE multicriteria methods. European Journal of Operational Research, 89:457-461, 1996.

[71] B. Kitchenham. Procedures for performing systematic reviews. Technical report, Software Engineering Group, Department of Computer Science Keele University (UK) and Empirical Software Engineering, National ICT Australia, 2004.

[72] B. Kitchenham and S. L. Pfleeger. Software quality: The elusive target. IEEE Software, 13(1):12-21, 1996.

[73] J. Kontio. A case study in applying a systematic method for COTS selection. In Proceedings of the 18th International Conference on Software Engineering (ICSE96). Berlin, Germany, pages 201-209, 1996.

[74] U. Küster and B. König-Ries. Measures for benchmarking semantic web service matchmaking correctness. In Proceedings of the 7th Extended Semantic Web Conference (ESWC2010). Heraklion, Crete, Greece, pages 45-59. Springer, 2010.

[75] V. S. Lai, R. P. Trueblood, and B. K. Wong. Software selection: A case study of the application of the Analytical Hierarchical Process to the selection of a multimedia authoring system. Information \& Management, 36:221-232, 1999.

[76] V. S. Lai, B. K. Wong, and W. Cheung. Group decision making in a multiple criteria environment: A case using the AHP in software selection. European Journal of Operational Research, 137(1):134-144, 2002.

[77] P. Lambrix, M. Habbouche, and M. Pérez. Evaluation of ontology development tools for bioinformatics. Bioinformatics, 19(12):1564, 2003.

[78] N. Li, L. Cabral, and S. Tymaniuk. D14.6: Results of the second evaluation of semantic Web service tools. Technical report, SEALS Consortium, June 2012. 
[79] S. K. Liang and C. T. Lien. Selecting the optimal ERP software by combining the ISO 9126 standard and fuzzy AHP approach. Contemporary Management Research, 3(1):23, 2006.

[80] C. T. Lien and S. K. Liang. An ERP system selection model with project management viewpoint - A fuzzy multi-criteria decision-making approach. International Journal of the Information Systems for Logistics and Management, 1(1):39-46, 2005.

[81] R. Likert. A technique for the measurement of attitudes. Archives of Psychology, 140:1-55, 1932.

[82] H. Y. Lin and P. Y. Hsu. Application of the Analytic Hierarchy Process on data warehouse system selection decisions for small and large enterprises in Taiwan. International Journal of Computer, the Internet and Management, 15 (3):73-93, 2007.

[83] A. Lozano-Tello and A. Gómez-Pérez. BAREMO: How to choose the appropriate software component using the Analytic Hierarchy Process. In Proceedings of the 14th International Conference on Software Engineering and Knowledge Engineering (SEKE2002). Ischia, Italy, pages 781-788, 2002.

[84] C. Macharis, J. Springael, K. De Brucker, and A. Verbeke. PROMETHEE and AHP: The design of operational synergies in multicriteria analysis. Strengthening PROMETHEE with ideas of AHP. European Journal of Operational Research, 153(2):307-317, 2004.

[85] J. M. Martel and B. Matarazzo. Other outranking approaches. In Multiple criteria decision analysis: State of the art surveys, pages 197-262. Springer, 2005.

[86] M. W. Mastalerz. ELECTRE method for choosing an e-learning platform. In Proceedings of 6th International Conference on Perspective Technologies and Methods in MEMS Design (MEMSTECH). Polyana, Ukraine, pages 168-171, 2010 .

[87] G. Meditskos and N. Bassiliades. Combining a DL reasoner and a rule engine for improving entailment-based OWL reasoning. In Proceedings of the rth International Semantic Web Conference (ISWC2008). Karlsruhe, Germany, pages 277-292. 2008.

[88] C. Meilicke, J. L. Aguirre-Cervantes, J. Euzenat, O. Šváb Zamazal, E. JiménezRuiz, I. Horrocks, and C. Trojahn. D12.6: Results of the second evaluation of matching tools. Technical report, SEALS Consortium, June 2012. 
[89] M. Mochól. The methodology for finding suitable ontology matching approaches. PhD thesis, Freie Universität Berlin, Germany, 2009.

[90] D. Morera. COTS evaluation using Desmet methodology \& Analytic Hierarchy Process (AHP). In Proceedings of the 4th International Conference on Product Focused Software Process Improvement. Rovaniemi, Finland, pages 485-493, 2002.

[91] M. Morisio and A. Tsoukias. IusWare: A methodology for the evaluation and selection of software products. IEEE Proceedings - Software Engineering., 144 (3):162-174, 1997.

[92] J. A. W. Mulebeke and L. Zheng. Analytical Network Process for software selection in product development: A case study. Journal of Engineering and Technology Management, 23(4):337-352, 2006.

[93] E. W. T. Ngai and E. W. C. Chan. Evaluation of knowledge management tools using AHP. Expert Systems with Applications, 29:889-899, 2005.

[94] X. Niu, H. Wang, G. Wu, G. Qi, and Y. Yu. Evaluating the stability and credibility of ontology matching methods. In Proceedings of the 8th Extended Semantic Web Conference (ESWC2011). Heraklion, Crete, Greece, pages 275289. Springer, 2011.

[95] OntoWeb. OntoWeb Deliverable 1.3: A survey on ontology tools. Technical report, IST OntoWeb Thematic Network, May 2002.

[96] Inc. O'Reilly Media and MediaLive International, editors. The Web 2.0 Conference. San Francisco, California, USA. October 5-7, 2004.

[97] W. Ossadnik and O. Lange. AHP-based evaluation of AHP-software. European Journal of Operational Research, 118(3):578-588, 1999.

[98] J. Otamendi, J. M. Pastor, and A. García. Selection of the simulation software for the management of the operations at an international airport. Simulation Modelling Practice and Theory, 16(8):1103-1112, 2008.

[99] I. Padayachee, P. Kotzé, and A. van Der Merwe. ISO 9126 external systems quality characteristics, sub-characteristics and domain specific criteria for evaluating e-Learning systems. In Proceedings of The Southern African Computer Lecturers' Association. University of Pretoria, South Africa, 2010.

[100] G. E. Phillips-Wren, E. D. Hahn, and G. A. Forgionne. A multiple-criteria framework for evaluation of decision support systems. Omega, 32:323-332, 2004. 
[101] M. Poveda-Villalón. Ontology evaluation: A pitfall-based approach to ontology diagnosis. PhD thesis, Universidad Politécnica de Madrid, Madrid, 2016.

[102] M. Poveda-Villalón, A. Gómez-Pérez, and M. C. Suárez-Figueroa. OOPS! (OntOlogy Pitfall Scanner!): An on-line tool for ontology evaluation. International Journal on Semantic Web and Information Systems (IJSWIS), 10(2): 7-34, 2014.

[103] K. Raed, C. Ding, and C. H. Chi. An enhanced PROMETHEE model for QoS-based web service selection. In Proceedings of the IEEE International Conference on Services Computing (SCC2011). Washington DC, USA, pages 536-543, 2011.

[104] V. Ravi, R. Shankar, and M. K. Tiwari. Analyzing alternatives in reverse logistics for end-of-life computers: ANP and Balanced Scorecard approach. Computers and Industrial Engineering, 48(2):327-356, 2005.

[105] J. Razmi and M. S. Sangari. A hybrid multi-criteria decision making model for ERP system selection. In Proceedings of the 4 th International Conference on Information and Automation for Sustainability. Colombo, Sri Lanka, pages 489-495, 2008.

[106] H. Rijgersberg, M. van Assem, and J. Top. Ontology of units of measure and related concepts. Semantic Web Journal, 4(1):3-13, 2013.

[107] G. C. Roper-Lowe and J. A. Sharp. The Analytic Hierarchy Process and its application to an information technology decision. Journal of the Operational Research Society, 41(1):49-59, 1990.

[108] B. Roy. Classement et choix en présence de points de vue multiples. RAIRO Operations Research - Recherche Opérationnelle, 2:57-75, 1968.

[109] B. Roy. From optimisation to multicriteria decision aid: Three main operational attitudes. In Multiple Criteria Decision Making, pages 1-34. Springer Berlin Heidelberg, 1976.

[110] B. Roy and P. Bertier. La méthode ELECTRE II: une méthode de classement en prédence de critères multiples. Metra international, 1971.

[111] B. Roy and J. C. Hugonnard. Ranking of suburban line extension projects on the Paris metro system by a multicriteria method. Transportation Research Part A: General, 16(4):301-312, 1982. 
[112] T. L. Saaty. A scaling method for priorities in hierarchical structures. Journal of Mathematical Psychology, 15(3):234-281, 1977.

[113] T. L. Saaty. The Analytic Hierarchy Process: Planning, priority setting, resource allocation. McGraw-Hill, 1980.

[114] T. L. Saaty. Decision making with dependence and feedback: The Analytic Network Process. RWS Publications, Pitsburg, 1996.

[115] T. L. Saaty. Decision making - The Analytic Hierarchy and Network Processes (AHP/ANP). Journal of Systems Science and Systems Engineering, 13(1):1-35, 2004.

[116] T. L. Saaty. Fundamentals of the Analytic Network Process - Dependence and feedback in decision-making with a single network. Journal of Systems Science and Systems Engineering, 13(2):129-157, 2004.

[117] T. L. Saaty. Fundamentals of the Analytic Network Process - Multiple networks with benefits, costs, opportunities and risks. Journal of Systems Science and Systems Engineering, 13(3):348-379, 2004.

[118] T. L. Saaty. The Analytic Hierarchy and Analytic Network Processes for the measurement of intangible criteria and for decision-making. In Multiple Criteria Decision Analysis: State of the Art Surveys, pages 345-405. Springer New York, 2005.

[119] J. Sarkis and S. Talluri. Evaluating and selecting e-commerce software and communication systems for a supply chain. European Journal of Operational Research, 159(2):318-329, 2004.

[120] A. Seidmann and A. Arbel. An analytic approach for planning computerized office systems. Omega, 11(6):607-617, 1983.

[121] A. Seidmann and A. Arbel. Microcomputer selection process for organizational information management. Information \&3 Management, 7(6):317-329, 1984.

[122] Y. J. Seo, H. Y. Jeong, and Y. J. Song. Best web service selection based on the decision making between QoS criteria of service. In Proceedings of the 2nd international conference on Embedded Software and Systems. Xi'an, China, pages 408-419. Springer-Verlag, 2005.

[123] Y. Y. Shih. A study of ERP systems selection via Fuzzy AHP method. In Proceedings of the 2nd International Symposium on Information Engineering and Electronic Commerce (IEEC). Ternopil, Ukraine, pages 1-4, 2010. 
[124] J. Spronk, R. E. Steuer, and C. Zopounidis. Multicriteria decision aid/analysis in finance. In Multiple criteria decision analysis: State of the Art surveys, pages 799-857. Springer, 2005.

[125] S. S. Stevens. On the theory of scales of measurement. Science, 103(2684): 677-680, 1946.

[126] X. Sun, V. Gollnick, Y. Li, and E. Stumpf. Intelligent multicriteria decision support system for systems design. Journal of Aircraft, 51:216-225, 2014.

[127] C. Tempich and R. Volz. Towards a benchmark for Semantic Web reasoners - An analysis of the DAML ontology library. In Proceedings of the 2nd International Workshop on Evaluation of Ontology-based Tools. Sanibel Island, Florida, USA, pages 1-12, 2003.

[128] M. Thirumaran, P. Dhavachelvan, S. Abarna, and S. Sheela. Parallel Analytic Hierarchy Process for Web service discovery and composition. In Proceedings of the 2011 International Conference on Recent Trends in Information Technology (ICRTIT). Chennai, India, pages 456-461, 2011.

[129] M. Toshtzar. Multi-criteria decision making approach to computer software evaluation: Application of the Analytical Hierarchy Process. Mathematical and Computer Modelling, 11:276-281, 1988.

[130] V. X. Tran, H. Tsuji, and R. Masuda. A new QoS ontology and its QoS-based ranking algorithm for Web services. Simulation Modelling Practice and Theory, 17(8):1378-1398, 2009.

[131] C. Trojahn, C. Meilicke, J. Euzenat, and H. Stuckenschmidt. Automating OAEI campaigns (first report). In Proceedings of the International Workshop on Evaluation of Semantic Technologies (IWEST2010). Shanghai, China, 2010.

[132] C. Trojahn, C. Meilicke, J. Euzenat, and O. Šváb Zamazal. D12.3: Results of the first evaluation of matching tools. Technical report, SEALS Consortium, November 2010.

[133] J. Urbani, S. Kotoulas, E. Oren, and F. van Harmelen. Scalable distributed reasoning using MapReduce. In Proceedings of the 8th International Semantic Web Conference (ISWC2009). Chantilly, Virginia, USA, pages 634-649. 2009.

[134] P. Vincke. L'Aide multicritère à la décision. Editions de l'Université de Bruxelles - Editions Ellispses, Bruxelles, 1989. English translation: Multicriteria decisionaid, Wiley, 1992. 
[135] I. Vlahavas, I. Stamelos, I. Refanidis, and A. Tsoukiàs. ESSE: An expert system for software evaluation. Knowledge-Based Systems, 12(4):183-197, 1999.

[136] C. C. Wei, C. F. Chien, and M. J. Wang. An AHP-based approach to ERP system selection. International Journal of Production Economics, 96(1):47-62, 2005 .

[137] S. N. Wrigley, K. Elbedweihy, D. Reinhard, A. Bernstein, and F. Ciravegna. D13.3: Results of the first evaluation of semantic search tools. Technical report, SEALS Consortium, November 2010.

[138] S. N. Wrigley, K. Elbedweihy, A. L. Gentile, V. Lanfranchi, and A. S. Dadzie. D13.6: Results of the second evaluation of semantic search tools. Technical report, SEALS Consortium, June 2012.

[139] S. N. Wrigley, R. García-Castro, and L. Nixon. Semantic Evaluation at Large Scale (SEALS). In Proceedings of the 21st International World Wide Web Conference (WWW2012), European projects track. Lyon, France, pages 299302. ACM, 2012.

[140] M. Yatskevich and F. Martín-Recuerda. D11.3: Results of the first evaluation of advanced reasoning systems. Technical report, SEALS Consortium, November 2010 .

[141] M. Yatskevich, I. Horrocks, F. Martín-Recuerda, and G. Stoilos. Storage and reasoning systems evaluation campaign 2010. In Proceedings of the International Workshop on Evaluation of Semantic Technologies (IWEST2010). Shanghai, China, 2010.

[142] M. Yatskevich, F. Martín-Recuerda, E. Jiménez-Ruiz, and M. Shneider. D11.6: Results of the second evaluation of storage and advanced reasoning systems. Technical report, SEALS Consortium, July 2012.

[143] E. K. Zavadskas and Z. Turskis. Multiple criteria decision making (MCDM) methods in economics: An overview. Technological and Economic Development of Economy, 17(2):397-427, 2011.

[144] H. Zulzalil, A. A. A. Ghani, M. H. Selamat, and R. Mahmod. A case study to identify quality attributes relationships for Web-based applications. International Journal of Computer Science and Network Security, 8(11):215-220, 2008 . 
[145] M. Zuo, S. Wang, and B. Wu. Research on Web services selection model based on AHP. In Proceedings of the IEEE International Conference on Service Operations and Logistics, and Informatics. Beijing, China, volume 2, pages 2763-2768, 2008. 


\section{Appendix A}

\section{List of Publications Analysed in the Semantic Technology Quality Specification Review}

Section 2.3.1 has presented a literature review on semantic technology quality specification in which one hundred publications were analysed; the details of this analysis have been made available in an online document ${ }^{1}$. Next, a list of all the publications from the literature review is provided in the same order as in the previously mentioned analysis document:

A1 S. Bail, B. Parsia, and U. Sattler. JustBench: A Framework for OWL Benchmarking. In Proceedings of the 9th International Semantic Web Conference, ISWC 2010, Shanghai, China. November 7-11, 2010, pp. 32-47

A2 B. Glimm, I. Horrocks, B. Motik, and G. Stoilos. Optimising Ontology Classification. In Proceedings of the 9th International Semantic Web Conference, ISWC 2010, Shanghai, China. November 7-11, 2010, pp. 225-240

A3 J. Du, G. Qi, J. Z. Pan, and Y. D. Shen. A Decomposition-Based Approach to Optimizing Conjunctive Query Answering in OWL DL. In Proceedings of the 8th International Semantic Web Conference, ISWC 2009, Chantilly, VA, USA, October 25-29, 2009, pp. 146-162

A4 F. Baader, M. Knechtel, and R. Peñaloza. A Generic Approach for Large-Scale Ontological Reasoning in the Presence of Access Restrictions to the Ontology's Axioms. In Proceedings of the 8th International Semantic Web Conference, ISWC 2009, Chantilly, VA, USA, October 25-29, 2009, pp. 49-64

\footnotetext{
${ }^{1}$ http://goo.gl/LzWuOT
} 
Appendix A. List of Publications Analysed in the Semantic Technology Quality 206

Specification Review

A5 F. Shi, J. Li, J. Tang, G. Xie, and H. Li. Actively Learning Ontology Matching via User Interaction. In Proceedings of the 8th International Semantic Web Conference, ISWC 2009, Chantilly, VA, USA, October 25-29, 2009, pp. 585-600

A6 J. L. Ambite, S. Darbha, A. Goel, C. A. Knoblock, K. Lerman, R. Parundekar, and T. Russ. Automatically Constructing Semantic Web Services from Online Sources. In Proceedings of the 8th International Semantic Web Conference, ISWC 2009, Chantilly, VA, USA, October 25-29, 2009, pp. 17-32

A7 A. Averbakh, D. Krause, and D. Skoutas. Exploiting User Feedback to Improve Semantic Web Service Discovery. In Proceedings of the 8th International Semantic Web Conference, ISWC 2009, Chantilly, VA, USA, October 25-29, 2009, pp. 33-48

A8 J. Urbani, S. Kotoulas, E. Oren, and F. van Harmelen. Scalable Distributed Reasoning Using MapReduce. In Proceedings of the 8th International Semantic Web Conference, ISWC 2009, Chantilly, VA, USA, October 25-29, 2009, pp. 634-649

A9 G. Meditskos and N. Bassiliades. Combining a DL Reasoner and a Rule Engine for Improving Entailment-Based OWL Reasoning. In Proceedings of the 7th International Semantic Web Conference, ISWC 2008, Karlsruhe, Germany, October 26-30, 2008, pp. 277-292

A10 D. Skoutas, D. Sacharidis, V. Kantere, and T. K. Sellis. Efficient Semantic Web Service Discovery in Centralized and P2P Environments. In Proceedings of the 7th International Semantic Web Conference, ISWC 2008, Karlsruhe, Germany, October 26-30, 2008, pp. 583-598

A11 D. Wei, T. Wang, J. Wang, and Y. Chen. Extracting Semantic Constraint from Description Text for Semantic Web Service Discovery. In Proceedings of the 7th International Semantic Web Conference, ISWC 2008, Karlsruhe, Germany, October 26-30, 2008, pp. 146-161

A12 M. Stollberg, M. Hepp, and J. Hoffmann. A Caching Mechanism for Semantic Web Service Discovery. In Proceedings of the 6th International Semantic Web Conference, ISWC 2007, Busan, Korea, November 11-15, 2007, pp. 480-493

A13 H. Tan and P. Lambrix. A Method for Recommending Ontology Alignment Strategies. In Proceedings of the 6th International Semantic Web Conference, ISWC 2007, Busan, Korea, November 11-15, 2007, pp. 494-507 
A14 A. Kalyanpur, B. Parsia, M. Horridge, and E. Sirin. Finding All Justifications of OWL DL Entailments. In Proceedings of the 6th International Semantic Web Conference, ISWC 2007, Busan, Korea, November 11-15, 2007, pp. 267-280

A15 B Cuenca Grau, C. Halaschek-Wiener, and Y. Kazakov. History Matters: Incremental Ontology Reasoning Using Modules. In Proceedings of the 6th International Semantic Web Conference, ISWC 2007, Busan, Korea, November 11-15, 2007, pp. 183-196

A16 L. Zhang, Q. L. Liu, J. Zhang, H. F. Wang, Y. Pan, and Y. Yu. Semplore: An IR Approach to Scalable Hybrid Query of Semantic Web Data. In Proceedings of the 6th International Semantic Web Conference, ISWC 2007, Busan, Korea, November 11-15, 2007, pp. 652-665

A17 Q. Zhou, C. Wang, M. Xiong, H. Wang, and Y. Yu. SPARK: Adapting Keyword Query to Semantic Search. In Proceedings of the 6th International Semantic Web Conference, ISWC 2007, Busan, Korea, November 11-15, 2007, pp. 694-707

A18 T. Gardiner, D. Tsarkov, and I. Horrocks. Framework for an Automated Comparison of Description Logic Reasoners. In Proceedings of the 5th International Semantic Web Conference, ISWC 2006, Athens, GA, USA, November 5-9, 2006, pp. 654-667

A19 U. Basters and M. Klusch. RS2D: Fast Adaptive Search for Semantic Web Services in Unstructured P2P Networks. In Proceedings of the 5th International Semantic Web Conference, ISWC 2006, Athens, GA, USA, November 5-9, 2006, pp. $87-100$

A20 G. Stoilos, G. B. Stamou, and S. D. Kollias. A String Metric for Ontology Alignment. In Proceedings of the 4th International Semantic Web Conference, ISWC 2005, Galway, Ireland, November 6-10, 2005, pp. 624-637

A21 M. Ehrig, S. Staab, and Y. Sure. Bootstrapping Ontology Alignment Methods with APFEL. In Proceedings of the 4th International Semantic Web Conference, ISWC 2005, Galway, Ireland, November 6-10, 2005, pp. 186-200

A22 R. García-Castro and A. Gómez-Pérez. Guidelines for Benchmarking the Performance of Ontology Management APIs. In Proceedings of the 4th International Semantic Web Conference, ISWC 2005, Galway, Ireland, November 6-10, 2005, pp. 277-292

A23 P. Mitra, N. F. Noy, and A. R. Jaiswal. OMEN: A Probabilistic Ontology Mapping Tool. In Proceedings of the 4th International Semantic Web Conference, ISWC 2005, Galway, Ireland, November 6-10, 2005, pp. 537-547 
Appendix A. List of Publications Analysed in the Semantic Technology Quality

A24 A. Bernstein, E. Kaufmann, A. Göhring, and C. Kiefer. Querying Ontologies: A Controlled English Interface for End-Users. In Proceedings of the 4th International Semantic Web Conference, ISWC 2005, Galway, Ireland, November 6-10, 2005, pp. 112-126

A25 U. Küster and B. König-Ries. Measures for Benchmarking Semantic Web Service Matchmaking Correctness. In Proceedings of the 7th Extended Semantic Web Conference, ESWC 2010, Heraklion, Crete, Greece, May 30 - June 3, 2010, pp. 45-59

A26 M. Junghans, S. Agarwal, and R. Studer. Towards Practical Semantic Web Service Discovery. In Proceedings of the 7th Extended Semantic Web Conference, ESWC 2010, Heraklion, Crete, Greece, May 30 - June 3, 2010, pp. 15-29

A27 M. Klusch, P. Kapahnke, and I. Zinnikus. Hybrid Adaptive Web Service Selection with SAWSDL-MX and WSDL-Analyzer. In Proceedings of the 6th European Semantic Web Conference, ESWC 2009, Heraklion, Crete, Greece, May 31 - June 4, 2009, pp. 550-564

A28 K. Eckert, C. Meilicke, and H. Stuckenschmidt. Improving Ontology Matching Using Meta-level Learning. In Proceedings of the 6th European Semantic Web Conference, ESWC 2009, Heraklion, Crete, Greece, May 31 - June 4, 2009, pp. 158-172

A29 P. Lambrix and Q. Liu. Using Partial Reference Alignments to Align Ontologies. In Proceedings of the 6th European Semantic Web Conference, ESWC 2009, Heraklion, Crete, Greece, May 31 - June 4, 2009, pp. 188-202

A30 R. Bhagdev, S. Chapman, F. Ciravegna, V. Lanfranchi, and D. Petrelli. Hybrid Search: Effectively Combining Keywords and Semantic Searches. In Proceedings of the 5th European Semantic Web Conference, ESWC 2008, Tenerife, Canary Islands, Spain, June 1-5, 2008, pp. 554-568

A31 L. Hollink, M. van Assem, S. Wang, A. Isaac, and G. Schreiber. Two Variations on Ontology Alignment Evaluation: Methodological Issues. In Proceedings of the 5th European Semantic Web Conference, ESWC 2008, Tenerife, Canary Islands, Spain, June 1-5, 2008, pp. 388-401

A32 A. Heß. An Iterative Algorithm for Ontology Mapping Capable of Using Training Data. In Proceedings of the 3rd European Semantic Web Conference, ESWC 2006, Budva, Montenegro, June 11-14, 2006, pp. 19-3 
A33 R. García-Castro and A. Gómez-Pérez. Benchmark Suites for Improving the RDF(S) Importers and Exporters of Ontology Development Tools. In Proceedings of the 3rd European Semantic Web Conference, ESWC 2006, Budva, Montenegro, June 11-14, 2006, pp. 155-169

A34 U. Straccia and R. Troncy. Towards Distributed Information Retrieval in the Semantic Web: Query Reformulation Using the oMAP Framework. In Proceedings of the 3rd European Semantic Web Conference, ESWC 2006, Budva, Montenegro, June 11-14, 2006, pp. 378-392

A35 D. Vallet, M. Fernández, and P. Castells. An Ontology-Based Information Retrieval Model. In Proceedings of the 2nd European Semantic Web Conference, ESWC 2005, Heraklion, Crete, Greece, May 29 - June 1, 2005, pp. 455-470

A36 P. Groot, H. Stuckenschmidt, and H. Wache. Approximating Description Logic Classification for SemanticWeb Reasoning. In Proceedings of the 2nd European Semantic Web Conference, ESWC 2005, Heraklion, Crete, Greece, May 29 June 1, 2005, pp. 318-332

A37 F. Giunchiglia, M. Yatskevich, and E. Giunchiglia. Efficient Semantic Matching. In Proceedings of the 2nd European Semantic Web Conference, ESWC 2005, Heraklion, Crete, Greece, May 29 - June 1, 2005, pp. 272-289

A38 M. Ehrig and Y. Sure. Ontology Mapping - An Integrated Approach. In Proceedings of the 1st European Semantic Web Symposium, ESWS 2004, Heraklion, Crete, Greece, May 10-12, 2004, pp. 76-91

A39 F. Giunchiglia, P. Shvaiko, and M. Yatskevich. S-Match: An Algorithm and an Implementation of Semantic Matching. In Proceedings of the 1st European Semantic Web Symposium, ESWS 2004, Heraklion, Crete, Greece, May 10-12, 2004, pp. 61-75

A40 A. Isaac, D. Kramer, L. van der Meij, S. Wang, S. Schlobach, and J. Stapel. Vocabulary Matching for Book Indexing Suggestion in Linked Libraries - A Prototype Implementation and Evaluation. In Proceedings of the 8th International Semantic Web Conference, ISWC 2009, Chantilly, VA, USA, October 25-29, 2009, pp. 843-859

A41 P. Klinov and B. Parsia. Optimization and Evaluation of Reasoning in Probabilistic Description Logic: Towards a Systematic Approach. In Proceedings of the 7th International Semantic Web Conference, ISWC 2008, Karlsruhe, Germany, October 26-30, 2008, pp. 213-228 
Appendix A. List of Publications Analysed in the Semantic Technology Quality

A42 S. Wang, G. Englebienne, and S. Schlobach. Learning Concept Mappings from Instance Similarity. In Proceedings of the 7th International Semantic Web Conference, ISWC 2008, Karlsruhe, Germany, October 26-30, 2008, pp. 339-355

A43 A. Isaac, L. van der Meij, S. Schlobach, and S. Wang. An Empirical Study of Instance-Based Ontology Matching. In Proceedings of the 6th International Semantic Web Conference, ISWC 2007, Busan, Korea, November 11-15, 2007, pp. $253-266$

A44 C. Patel, J. Cimino, J. Dolby, A. Fokoue, A. Kalyanpur, A. Kershenbaum, L. Ma, E. Schonberg, and K. Srinivas. Matching Patient Records to Clinical Trials Using Ontologies. In Proceedings of the 6th International Semantic Web Conference, ISWC 2007, Busan, Korea, November 11-15, 2007, pp. 816-829

A45 P. Avesani, F. Giunchiglia, and M. Yatskevich. A Large Scale Taxonomy Mapping Evaluation. In Proceedings of the 4th International Semantic Web Conference, ISWC 2005, Galway, Ireland, November 6-10, 2005, pp. 67-81

A46 W. R. van Hage, S. Katrenko, and G. Schreiber. A Method to Combine Linguistic Ontology-Mapping Techniques. In Proceedings of the 4th International Semantic Web Conference, ISWC 2005, Galway, Ireland, November 6-10, 2005, pp. $732-744$

A47 B. Benatallah, M. S. Hacid, C. Rey, and F. Toumani. Request Rewriting-Based Web Service Discovery. In Proceedings of the 2nd International Semantic Web Conference, ISWC 2003, Sanibel Island, FL, USA, October 20-23, 2003, pp. $242-257$

A48 D. Tsarkov, A. Riazanov, S. Bechhofer, and I. Horrocks. Using Vampire to Reason with OWL. In Proceedings of the 3rd International Semantic Web Conference, ISWC 2004, Hiroshima, Japan, November 7-11, 2004, pp. 471-485

A49 J. Euzenat. An API for Ontology Alignment. In Proceedings of the 3rd International Semantic Web Conference, ISWC 2004, Hiroshima, Japan, November 7-11, 2004, pp. 698-712

A50 A. Tordai, J. van Ossenbruggen, G. Schreiber, and B. Wielinga. Aligning Large SKOS-Like Vocabularies: Two Case Studies. In Proceedings of the 7th Extended Semantic Web Conference, ESWC 2010, Heraklion, Crete, Greece, May 30 - June 3, 2010, pp. 198-212

A51 M. Luther, T. Liebig, S. Böhm, and O. Noppens. Who the Heck Is the Father of Bob? A Survey of the OWL Reasoning Infrastructure for Expressive Real-World 
Applications. In Proceedings of the 6th European Semantic Web Conference, ESWC 2009, Heraklion, Crete, Greece, May 31 - June 4, 2009, pp. 66-80

A52 V. Spiliopoulos, A. G. Valarakos, and G. A. Vouros. CSR: Discovering Subsumption Relations for the Alignment of Ontologies. In Proceedings of the 5th European Semantic Web Conference, ESWC 2008, Tenerife, Canary Islands, Spain, June 1-5, 2008, pp. 418-431

A53 T. Weithöner, T. Liebig, M. Luther, S. Böhm, F. Von Henke, and O. Noppens. Real-World Reasoning with OWL. In Proceedings of the 4th European Semantic Web Conference, ESWC 2007, Innsbruck, Austria, June 3-7, 2007, pp. 296-310

A54 L. Ma, Y. Yang, Z. Qiu, G. Xie, Y. Pan, and S. Liu. Towards a Complete OWL Ontology Benchmark. In Proceedings of the 3rd European Semantic Web Conference, ESWC 2006, Budva, Montenegro, June 11-14, 2006, pp. 125-139

A55 M. Klusch and P. Kapahnke. iSeM: Approximated Reasoning for Adaptive Hybrid Selection of Semantic Services. In Proceedings of the 7th Extended Semantic Web Conference, ESWC 2010, Heraklion, Crete, Greece, May 30 June 3, 2010, pp. 30-44

A56 P. Jain, P. Z. Yeh, K. Verma, R. G. Vasquez, M. Damova, P. Hitzler, and A. P. Sheth. Contextual Ontology Alignment of LOD with an Upper Ontology: A Case Study with Proton. In Proceedings of the 8th Extended Semantic Web Conference, ESWC 2011, Heraklion, Crete, Greece, May 29 - June 2, 2011, pp. 80-92

A57 X. Niu, H. Wang, G. Wu, G. Qi, and Y. Yu. Evaluating the Stability and Credibility of Ontology Matching Methods. In Proceedings of the 8th Extended Semantic Web Conference, ESWC 2011, Heraklion, Crete, Greece, May 29 June 2, 2011, pp. 275-289

A58 I. Kollia, B. Glimm, and I. Horrocks. SPARQL Query Answering over OWL Ontologies. In Proceedings of the 8th Extended Semantic Web Conference, ESWC 2011, Heraklion, Crete, Greece, May 29 - June 2, 2011, pp. 382-396

A59 A. Mehdi, S. Rudolph, and S. Grimm. Epistemic Querying of OWL Knowledge Bases. In Proceedings of the 8th Extended Semantic Web Conference, ESWC 2011, Heraklion, Crete, Greece, May 29 - June 2, 2011, pp. 397-409

A60 A. Ferrara, S. Montanelli, J. Noessner, and H. Stuckenschmidt. Benchmarking Matching Applications on the Semantic Web. In Proceedings of the 8th Extended Semantic Web Conference, ESWC 2011, Heraklion, Crete, Greece, May 29 - June 2, 2011, pp. 108-122 
Appendix A. List of Publications Analysed in the Semantic Technology Quality 212

Specification Review

A61 M. Salvadores, G. Correndo, S. Harris, N. Gibbins, and N. Shadbolt. The Design and Implementation of Minimal RDFS Backward Reasoning in 4store. In Proceedings of the 8th Extended Semantic Web Conference, ESWC 2011, Heraklion, Crete, Greece, May 29 - June 2, 2011, pp. 139-153

A62 M. Sah and V. Wade. A Novel Concept-Based Search for the Web of Data Using UMBEL and a Fuzzy Retrieval Model. In Proceedings of the 9th Extended Semantic Web Conference, ESWC 2012, Heraklion, Crete, Greece, May 27-31, 2012, pp. 103-118

A63 T. Ruotsalo. Domain Specific Data Retrieval on the Semantic Web. In Proceedings of the 9th Extended Semantic Web Conference, ESWC 2012, Heraklion, Crete, Greece, May 27-31, 2012, pp. 422-436

A64 C. Stab, K. Nazemi, M. Breyer, D. Burkhardt, and J. Kohlhammer. Semantics Visualization for Fostering Search Result Comprehension. In Proceedings of the 9th Extended Semantic Web Conference, ESWC 2012, Heraklion, Crete, Greece, May 27-31, 2012, pp. 633-646

A65 R. Blanco, P. Mika, and S. Vigna. Effective and Efficient Entity Search in RDF Data. In Proceedings of the 10th International Semantic Web Conference, ISWC 2011, Bonn, Germany, October 23-27, 2011, pp. 83-97

A66 E. Jiménez-Ruiz and B. Cuenca Grau. LogMap Logic-Based and Scalable Ontology Matching. In Proceedings of the 10th International Semantic Web Conference, ISWC 2011, Bonn, Germany, October 23-27, 2011, pp. 273-288

A67 C. Seitz and R. Schönfelder. Rule-Based OWL Reasoning for Specific Embedded Devices. In Proceedings of the 10th International Semantic Web Conference, ISWC 2011, Bonn, Germany, October 23-27, 2011, pp. 237-252

A68 S. Ferré and A. Hermann. Semantic Search Reconciling Expressive Querying and Exploratory Search. In Proceedings of the 10th International Semantic Web Conference, ISWC 2011, Bonn, Germany, October 23-27, 2011, pp. 177-192

A69 S. Rong, X. Niu, E. W. Xiang, H. Wang, Q. Yang, and Y. Yu. A Machine Learning Approach for Instance Matching Based on Similarity Metrics. In Proceedings of the 11th International Semantic Web Conference, ISWC 2012, Boston, MA, USA, November 11-15, 2012, pp. 460-475

A70 Y. Tzitzikas, C. Lantzaki, and D. Zeginis. Blank Node Matching and RDFS Comparison Functions. In Proceedings of the 11th International Semantic Web Conference, ISWC 2012, Boston, MA, USA, November 11-15, 2012, pp. 591-607 
A71 N. Aggarwal. Cross Lingual Semantic Search by Improving Semantic Similarity and Relatedness Measures. In Proceedings of the 11th International Semantic Web Conference, ISWC 2012, Boston, MA, USA, November 11-15, 2012, pp. 375-382

A72 K. Slabbekoorn, L. Hollink, and G. J. Houben. Domain-Aware Ontology Matching. In Proceedings of the 11th International Semantic Web Conference, ISWC 2012, Boston, MA, USA, November 11-15, 2012, pp. 542-558

A73 S. Duan, A. Fokoue, O. Hassanzadeh, A. Kementsietsidis, K. Srinivas, and M. J. Ward. Instance-Based Matching of Large Ontologies Using Locality-Sensitive Hashing. In Proceedings of the 11th International Semantic Web Conference, ISWC 2012, Boston, MA, USA, November 11-15, 2012, pp. 49-64

A74 A. Armas Romero, B. Cuenca Grau, and I. Horrocks. MORe: Modular Combination of OWL Reasoners for Ontology Classification. In Proceedings of the 11th International Semantic Web Conference, ISWC 2012, Boston, MA, USA, November 11-15, 2012, pp. 1-16

A75 R. S. Gonçalves, B. Parsia, and U. Sattler. Performance Heterogeneity and Approximate Reasoning in Description Logic Ontologies. In Proceedings of the 11th International Semantic Web Conference, ISWC 2012, Boston, MA, USA, November 11-15, 2012, pp. 82-98

A76 N. Heino and J. Z. Pan. RDFS Reasoning on Massively Parallel Hardware. In Proceedings of the 11th International Semantic Web Conference, ISWC 2012, Boston, MA, USA, November 11-15, 2012, pp. 133-148

A77 N. F. Noy and M. A. Musen. Evaluating Ontology-Mapping Tools: Requirements and Experience. In Proceedings of the 1st Workshop on Evaluation of Ontology-based Tools, EON 2002, held within the 13th International Conference on Knowledge Engineering and Knowledge Management, Sigüenza, Spain, September 30, 2002, pp. 1-14

A78 N. Sugiura, M. Kurematsu, N. Fukuta, N. Izumi, and T. Yamaguchi. A Domain Ontology Engineering Tool With General Ontologies and Text Corpus. In Proceedings of the 2nd Workshop on Evaluation of Ontology-based Tools, EON 2003, held within the 2nd International Semantic Web Conference, Sanibel Island, Florida, USA, October 20, 2003

A79 O. Corcho, A. Gómez-Pérez, D. J. Guerrero-Rodríguez, D. Pérez-Rey, A. RuizCristina, T. Sastre-Toral, and M. C. Suárez-Figueroa. Evaluation Experiment of Ontology Tools' Interoperability with the WebODE Ontology Engineering 
Appendix A. List of Publications Analysed in the Semantic Technology Quality

Workbench. In Proceedings of the 2nd Workshop on Evaluation of Ontologybased Tools, EON 2003, held within the 2nd International Semantic Web Conference, Sanibel Island, Florida, USA, October 20, 2003

A80 C. Tempich and R. Volz. Towards a Benchmark for Semantic Web Reasoners - An Analysis of the DAML Ontology Library. In Proceedings of the 2nd Workshop on Evaluation of Ontology-based Tools, EON 2003, held within the 2nd International Semantic Web Conference, Sanibel Island, Florida, USA, October 20, 2003

A81 R. Troncy, A. Isaac, and V. Malaisé. Using XSLT for Interoperability DOE and The Travelling Domain Experiment. In Proceedings of the 2nd Workshop on Evaluation of Ontology-based Tools, EON 2003, held within the 2nd International Semantic Web Conference, Sanibel Island, Florida, USA, October 20, 2003

A82 R. García-Castro and A. Gómez-Pérez. A Benchmark Suite for Evaluating the Performance of the WebODE Ontology Engineering Platform. In Proceedings of the 3rd International Workshop on Evaluation of Ontology-based Tools, EON 2004, held within the 3rd International Semantic Web Conference, Hiroshima, Japan, November 8, 2004

A83 T. Morita, Y. Shigeta, N. Sugiura, N. Fukuta, N. Izumi, and T. Yamaguchi. DOODLE-OWL: OWL-based Semi-Automatic Ontology Development Environment. In Proceedings of the 3rd International Workshop on Evaluation of Ontology-based Tools, EON 2004, held within the 3rd International Semantic Web Conference, Hiroshima, Japan, November 8, 2004

A84 M. Ehrig and Y. Sure. Ontology Alignment - Karlsruhe. In Proceedings of the 3rd International Workshop on Evaluation of Ontology-based Tools, EON 2004, held within the 3rd International Semantic Web Conference, Hiroshima, Japan, November 8, 2004

A85 J. Euzenat, D. Loup, M. Touzani, and P. Valtchev. Ontology Alignment with OLA. In Proceedings of the 3rd International Workshop on Evaluation of Ontology-based Tools, EON 2004, held within the 3rd International Semantic Web Conference, Hiroshima, Japan, November 8, 2004

A86 A. N. Chitnis, A. Qasem, and J. Heflin. Benchmarking Reasoners for MultiOntology Applications. In Proceedings of the 5th International Workshop on Evaluation of Ontologies and Ontology-based Tools, EON 2007, held within the 6th International Semantic Web Conference, Busan, Korea, November 17, 2007 
A87 W. R. van Hage, A. Isaac, and Z. Aleksovski. Sample Evaluation of OntologyMatching Systems. In Proceedings of the 5th International Workshop on Evaluation of Ontologies and Ontology-based Tools, EON 2007, held within the 6th International Semantic Web Conference, Busan, Korea, November 17, 2007

A88 C. Trojahn, C. Meilicke, J. Euzenat, and H. Stuckenschmidt. Automating OAEI Campaigns (First Report). In Proceedings of the 1st International Workshop on Evaluation of Semantic Technologies, IWEST 2010, held within the 9th International Semantic Web Conference, Shanghai, China, November 8, 2010

A89 H. Halpin, D. M. Herzig, P. Mika, R. Blanco, J. Pound, H. S. Thompson, and T. T. Duc. Evaluating Ad-Hoc Object Retrieval. In Proceedings of the 1st International Workshop on Evaluation of Semantic Technologies, IWEST 2010, held within the 9th International Semantic Web Conference, Shanghai, China, November 8, 2010

A90 S. N. Wrigley, K. Elbedweihy, D. Reinhard, A. Bernstein, and F. Ciravegna. Evaluating Semantic Search Tools using the SEALS platform. In Proceedings of the 1st International Workshop on Evaluation of Semantic Technologies, IWEST 2010, held within the 9th International Semantic Web Conference, Shanghai, China, November 8, 2010

A91 M. Yatskevich, I. Horrocks, F. Martin-Recuerda, and G. Stoilos. Storage and Reasoning Systems Evaluation Campaign 2010. In Proceedings of the 1st International Workshop on Evaluation of Semantic Technologies, IWEST 2010, held within the 9th International Semantic Web Conference, Shanghai, China, November 8, 2010

A92 K. Elbedweihy, S. N. Wrigley, F. Ciravegna, D. Reinhard, and A. Bernstein. Evaluating Semantic Search Systems to Identify Future Directions of Research. In Proceedings of the 2nd International Workshop on Evaluation of Semantic Technologies, IWEST 2012, held within the 9th Extended Semantic Web Conference, Heraklion, Greece, May 28, 2012

A93 C. Meilicke, C. Trojahn, O. Šváb-Zamazal, and D. Ritze. Multilingual Ontology Matching Evaluation - A First Report on using MultiFarm. In Proceedings of the 2nd International Workshop on Evaluation of Semantic Technologies, IWEST 2012, held within the 9th Extended Semantic Web Conference, Heraklion, Greece, May 28, 2012

A94 R. García-Castro and A. Gómez-Pérez. A Method for Performing an Exhaustive Evaluation of $\mathrm{RDF}(\mathrm{S})$ Importers. In Proceedings of the 1st International Workshop on Scalable Semantic Web Knowledge Base Systems, SSWS 2005, 
Appendix A. List of Publications Analysed in the Semantic Technology Quality

held within the the 6th International Conference on Web Information Systems and Engineering, New York City, NY, USA, November 20, 2005

A95 J. Dolby, A. Fokoue, A. Kalyanpur, L. Ma, C. Patel, E. Schonberg, K. Srinivas, and X. Sun. Efficient Reasoning on Large SHIN Aboxes in Relational Databases. In Proceedings of the 5th International Workshop on Scalable Semantic Web Knowledge Base Systems, SSWS 2009, held within the 8th International Semantic Web Conference, Washington DC, USA, October 26, 2009, pp. 110-124

A96 I. Tachmazidis, G. Antoniou, G. Flouris, and S. Kotoulas. Scalable Nonmonotonic Reasoning over RDF Data Using MapReduce. In Proceedings of the Joint Workshop on Scalable and High-Performance Semantic Web Systems, SSWS + HPCSW 2012, held within the 11th International Semantic Web Conference, Boston, USA, November 11, 2012, pp. 75-90

A97 A. Fokoue, A. Kershenbaum, L. Ma, C. Patel, E. Schonberg, and K. Srinivas. Using Abstract Evaluation in ABox Reasoning. In Proceedings of the 2nd International Workshop on Scalable Semantic Web Knowledge Base Systems, SSWS 2006, held within the 5th International Semantic Web Conference, Athens, Georgia, USA, November 5, 2006, pp. 61-74

A98 D. M. McRae-Spencer and N. R. Shadbolt. Semiometrics: Applying Ontologies across Large-Scale Digital Libraries. In Proceedings of the 2nd International Workshop on Scalable Semantic Web Knowledge Base Systems, SSWS 2006, held within the 5th International Semantic Web Conference, Athens, Georgia, USA, November 5, 2006, pp. 75-88

A99 F. Fuchs and M. Berger. Towards Scalable Retrieval of Distributed and Dynamic Ontology Instances. In Proceedings of the 2nd International Workshop on Scalable Semantic Web Knowledge Base Systems, SSWS 2006, held within the 5th International Semantic Web Conference, Athens, Georgia, USA, November 5, 2006, pp. 89-100

A100 T. Weithöner, T. Liebig, M. Luther, and S. Böhm. What's Wrong with OWL Benchmarks?. In Proceedings of the 2nd International Workshop on Scalable Semantic Web Knowledge Base Systems, SSWS 2006, held within the 5th International Semantic Web Conference, Athens, Georgia, USA, November 5, 2006, pp. 101-114 


\section{Appendix B}

\section{Detailed Description of the SemQuaRE Quality Model}

This appendix presents in detail the quality model obtained for all the evaluation campaigns performed in the SEALS project (Section 2.3.2) which covered: ontology engineering tools (Section B.1), reasoning systems (Section B.2), ontology matching tools (Section B.3), semantic search tools (Section B.4), and semantic web service tools (Section B.5).

In the final version of the model, the analysis presented in the flexibility evaluation of SemQuaRE (Section 7.4.2) has also been taken into account. Several characteristics that were not obtained from the SEALS evaluation campaigns are fully included into SemQuaRE, together with a set of related quality measures. Also, a new type of tool that was not evaluated in the SEALS project has been included into SemQuaRE, which is ontology annotation tools (Section B.6).

\section{B.1 Ontology Engineering Tools}

The evaluation campaign for ontology engineering tools contained three evaluation scenarios to evaluate the conformance (Figure B.1), interoperability (Figure B.2), and scalability (Figure B.3) of these tools.

\section{B.1.1 Test Data}

The test data used in the evaluation scenarios had the same structure. Different test suites were used, each containing several tests with one origin ontology each.

Origin Ontology - The ontology to be used as input 


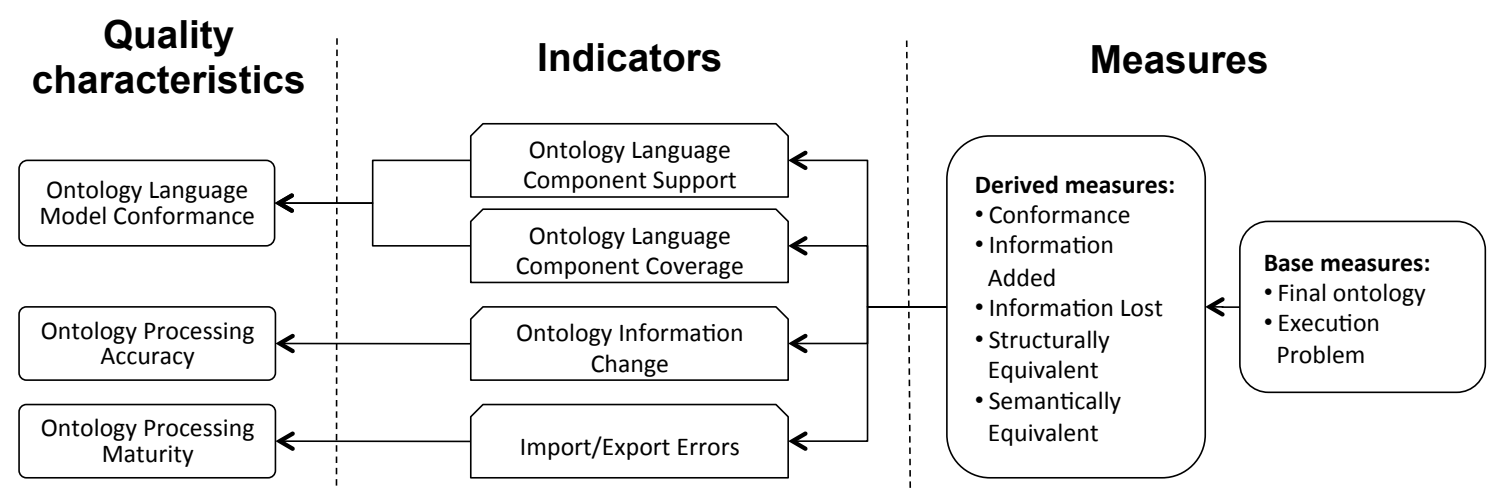

Figure B.1: Conformance scenario of ontology engineering tools.

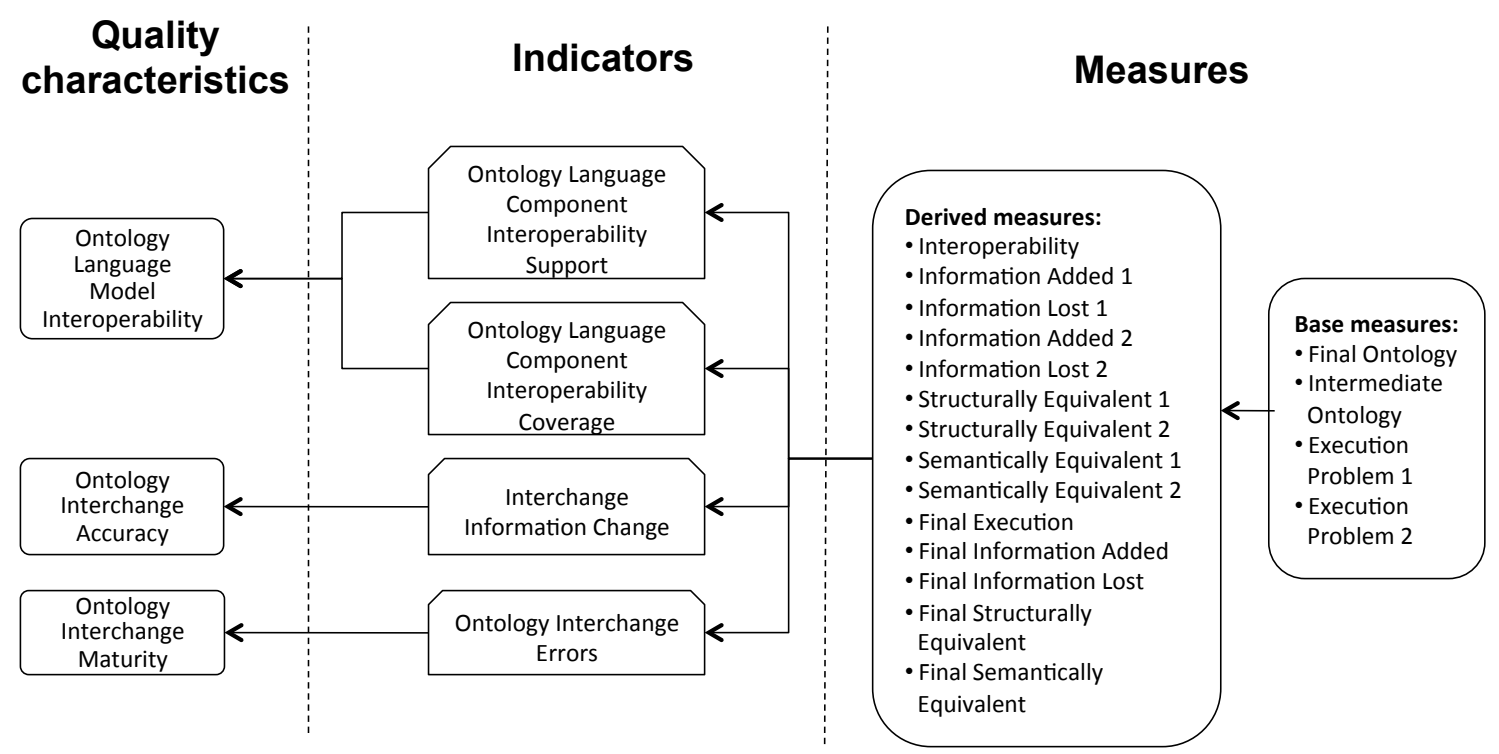

Figure B.2: Interoperability scenario of ontology engineering tools.

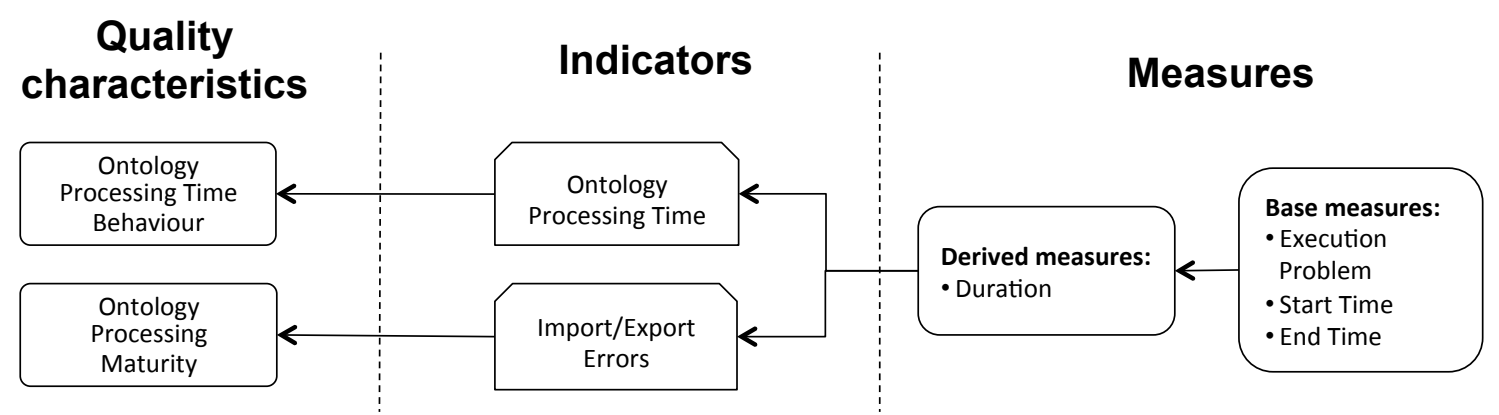

Figure B.3: Scalability scenario of ontology engineering tools. 


\section{B.1.2 Base Measures}

\section{Conformance Scenario}

Final Ontology - The ontology that is produced by the tool when importing and exporting the origin ontology

Execution Problem - Whether there was any execution problem in the tool when importing and exporting the origin ontology. Possible values are true, and false

\section{Interoperability Scenario}

Final Ontology - The ontology that is produced by the tool when importing and exporting the intermediate ontology

Intermediate Ontology - The ontology that is produced by the origin tool when importing and exporting the origin ontology

Execution Problem 1 - Whether there was any execution problem in the tool when importing and exporting the origin ontology. Possible values are true, and false

Execution Problem 2 - Whether there was any execution problem in the tool when importing and exporting the intermediate ontology. Possible values are true, and false

\section{Scalability Scenario}

Execution Problem - Whether there was any execution problem in the tool when importing and exporting the origin ontology. Possible values are true, and false

Start Time - The time when the operation of importing and exporting the origin ontology starts. Possible value is any number greater than 0

End Time - The time when the operation of importing and exporting the origin ontology ends. Possible value is any number greater than 0

\section{B.1.3 Derived Measures}

\section{Conformance Scenario}

Conformance - Whether the origin ontology has been imported and exported correctly with no addition or loss of information. Possible values are true, and false

$$
\text { Conformance }=\text { structurally equivalent } \wedge \neg(\text { execution problem })
$$

Information Added - The information added to the origin ontology (e.g., triples, axioms) after importing and exporting it. Possible values are null and concrete 
information in terms of triples

Information added $= \begin{cases}\text { null } & \text { if final ontology }=\text { origin ontology } \\ \text { final ontology - origin ontology } & \text { if final ontology } \neq \text { origin ontology }\end{cases}$

Information Lost - The information lost from the origin ontology (e.g., triples, axioms) after importing and exporting it. Possible values are null and concrete information in terms of triples

$$
\text { Information lost }= \begin{cases}\text { null } & \text { if final ontology }=\text { origin ontology } \\ \text { origin ontology }- \text { final ontology } & \text { if final ontology } \neq \text { origin ontology }\end{cases}
$$

Structurally Equivalent - Whether the origin ontology and the final one are structurally equivalent. Possible values are true, and false

Structurally equivalent $=($ information added $=$ null $) \wedge($ information lost $=$ null $)$

Semantically Equivalent - Whether the origin ontology and the final one are semantically equivalent. Possible values are true, and false

Semantically equivalent $=$

$$
=(\text { final ontology } \models \text { origin ontology }) \wedge(\text { origin ontology } \models \text { final ontology })
$$

\section{Interoperability Scenario}

Interoperability - Whether the origin ontology has been interchanged correctly with no addition or loss of information. Possible values are true, and false

$$
\text { Interoperability }=(\text { final structurally equivalent }) \wedge \neg(\text { final execution })
$$

Information Added 1 - The information added to the origin ontology after importing and exporting it. Possible values are null and concrete information in terms of triples

Information added $1= \begin{cases}\text { null } & \text { if intermediate ontology }=\text { origin ontology } \\ \text { intermediate ontology - origin ontology } & \text { if intermediate ontology } \neq \text { origin ontology }\end{cases}$

Information Lost 1 - The information lost from the origin ontology after importing 
and exporting it. Possible values are null and concrete information in terms of triples Information lost $1= \begin{cases}\text { null } & \text { if intermediate ontology }=\text { origin ontology } \\ \text { origin ontology - intermediate ontology } & \text { if intermediate ontology } \neq \text { origin ontology }\end{cases}$

Information Added 2 - The information added to the intermediate ontology after importing and exporting it. Possible values are null and concrete information in terms of triples

Information added $2= \begin{cases}\text { null } & \text { if intermediate ontology }=\text { final ontology } \\ \text { final ontology - intermediate ontology } & \text { if intermediate ontology } \neq \text { final ontology }\end{cases}$

Information Lost 2 - The information lost from the intermediate ontology after importing and exporting it. Possible values are null and concrete information in terms of triples

$$
\text { Information lost } 2= \begin{cases}\text { null } & \text { if intermediate ontology }=\text { final ontology } \\ \text { intermediate ontology }- \text { final ontology } & \text { if intermediate ontology } \neq \text { final ontology }\end{cases}
$$

Structurally Equivalent 1 - Whether the origin ontology and the intermediate one are structurally equivalent. Possible values are true, and false

Structurally equivalent $1=($ information added $1=$ null $) \wedge($ information lost $1=$ null $)$

Structurally Equivalent 2 - Whether the intermediate ontology and the final one are structurally equivalent. Possible values are true, and false

Structurally equivalent $2=($ information added $2=$ null $) \wedge($ information lost $2=$ null $)$

Semantically Equivalent 1 - Whether the origin ontology and the intermediate one are semantically equivalent. Possible values are true, and false

Semantically equivalent $=($ intermediate ontology $\models$ origin ontology $) \wedge$

$$
\wedge \text { (origin ontology } \models \text { intermediate ontology) }
$$

Semantically Equivalent 2 - Whether the intermediate ontology and the final one are semantically equivalent. Possible values are true, and false

$$
\begin{aligned}
\text { Semantically equivalent }= & (\text { intermediate ontology } \models \text { final ontology }) \wedge \\
& \wedge(\text { final ontology } \models \text { intermediate ontology })
\end{aligned}
$$

Final Execution - Whether there was any execution problem in the tool when 
importing and exporting the origin and intermediate ontology. Possible values are true, and false

Final execution $=($ execution problem 1$) \vee($ execution problem 2$)$

Final Information Added - The information added to the origin ontology after importing and exporting it by the first tool and then importing and exporting the intermediate ontology by the second tool. Possible values are null and concrete information in terms of triples

$$
\text { Final information added }= \begin{cases}\text { null } & \text { if final ontology }=\text { origin ontology } \\ \text { final ontology - origin ontology } & \text { if final ontology } \neq \text { origin ontology }\end{cases}
$$

Final Information Lost - The information lost from the origin ontology after importing and exporting it by the first tool and then importing and exporting the intermediate ontology by the second tool. Possible values are null and concrete information in terms of triples

$$
\text { Final information lost }= \begin{cases}\text { null } & \text { if final ontology }=\text { origin ontology } \\ \text { origin ontology }- \text { final ontology } & \text { if final ontology } \neq \text { origin ontology }\end{cases}
$$

Final Structurally Equivalent - Whether the origin ontology and the final one are structurally equivalent. Possible values are true, and false

Final structurally equivalent $=$

$=($ final information added $=$ null $) \wedge($ final information lost $=$ null $)=$

$=($ structurally equivalent 1$) \wedge($ structurally equivalent 2$)$

Final Semantically Equivalent - Whether the origin ontology and the final one are semantically equivalent. Possible values are true, and false

Final semantically equivalent $=$

$=($ semantically equivalent 1$) \wedge($ semantically equivalent 2$)$

\section{Scalability Scenario}

Duration - The amount of time needed for importing and exporting the origin ontology. Possible value is any number greater than 0

Duration $=$ end time - start time 


\section{B.1.4 Ontology Engineering Tools Indicators}

\section{Conformance Scenario}

Ontology Language Component Support - Whether the tool fully supports an ontology language component. Possible values true and false

Ontology language component support $=$

$$
=\left(\frac{\# \text { tests that contain the component } \mid(\text { conformance }=\text { true })}{\# \text { tests that contain the component }}=1\right)
$$

Ontology Language Component Coverage - The ratio of ontology components that are shared by a tool internal model and an ontology language model. Possible values are in the range from 0 to 100

Ontology language component coverage $=$

$$
=\frac{\# \text { components in the ontology language } \mid(\text { component support }=\text { true })}{\# \text { components in the ontology language }} \times 100
$$

Ontology Information Change - The ratio of information additions or losses when importing and exporting ontologies. Possible values are in the range from 0 to 100

Ontology information change $=$

$$
=\frac{\# \text { tests } \mid(\text { information added } \neq \text { null } \vee \text { information lost } \neq \text { null })}{\# \text { tests }} \times 100
$$

Import/Export Errors - The ratio of tool execution errors when importing and exporting ontologies. Possible values are in the range from 0 to 100

$$
\text { Import } / \text { Export errors }=\frac{\# \text { tests } \mid(\text { execution problem }=\text { true })}{\# \text { tests }} \times 100
$$

\section{Interoperability Scenario}

Ontology Language Component Interoperability Support - Whether the tool fully supports an ontology language component interchange. Possible values true and 
false

Ontology language component interoperability support $=$

$$
=\left(\frac{\# \text { tests that contain the component } \mid(\text { interoperability }=\text { true })}{\# \text { tests that contain the component }}=1\right)
$$

Ontology Language Component Interoperability Coverage - The ratio of ontology components that can be interchanged with other tools. Possible values are in the range from 0 to 100

Ontology language component interoperability coverage $=$

$$
=\frac{\# \text { components in the ontology language } \mid(\text { component interoperability support }=\text { true })}{\# \text { components in the ontology language }} \times 100
$$

Interchange Information Change - The ratio of information additions or losses when interchanging ontologies. Possible values are in the range from 0 to 100

Interchange information change $=$

$$
=\frac{\# \text { tests } \mid(\text { final information added } \neq \text { null } \vee \text { final information lost } \neq \text { null })}{\# \text { tests }} \times 100
$$

Ontology Interchange Errors - The ratio of tool execution errors when interchanging ontologies. Possible values are in the range from 0 to 100

$$
\text { Ontology interchange errors }=\frac{\# \text { tests } \mid(\text { final execution }=\text { true })}{\# \text { tests }} \times 100
$$

\section{Scalability Scenario}

Ontology Processing Time - The average amount of time needed for importing and exporting ontologies. Possible value is any number greater than 0

$$
\text { Ontology processing time }=\frac{\sum_{n} \text { duration in the } n^{\text {th }} \text { test }}{\# \text { tests }}
$$

Import/Export Errors - The ratio of tool execution errors when importing and 
exporting ontologies. Possible values are in the range from 0 to 100

$$
\text { Import } / \text { Export errors }=\frac{\# \text { tests } \mid(\text { execution problem }=\text { true })}{\# \text { tests }} \times 100
$$

\section{B.1.5 SquaRE Quality Characteristics}

Functional Suitability - Degree to which a product or system provides functions that meet stated and implied needs when used under specified conditions

Functional Compliance - Degree to which the software product adheres to standards, conventions or regulations in laws and similar prescriptions relating to functional suitability. Functional Compliance is introduced in SemQuaRE as a subcharacteristic of Functional Suitability

Functional Correctness - Degree to which a product or system provides the correct results with the needed degree of precision. Functional Correctness is a sub-characteristic of Functional Suitability

Compatibility - Degree to which a product, system or component can exchange information with other products, systems or components, and/or perform its required functions, while sharing the same hardware or software environment

Interoperability - Degree to which two or more systems, products or components can exchange information and use the information that has been exchanged. Interoperability is a sub-characteristic of Compatibility

Performance Efficiency - The performance relative to the amount of resources used under stated conditions

Time Behaviour - Degree to which the response, processing times and throughput rates of a product or system meet requirements when performing its functions. Time behaviour is a sub-characteristic of Performance Efficiency

Reliability - Degree to which a system, product or component performs specified functions under specified conditions for a specified period of time

Maturity - Degree to which a system, product or component meets needs for reliability under normal operation. Maturity is a sub-characteristic of Reliability

\section{B.1.6 Ontology Engineering Tools Quality Characteristics}

Ontology Language Model Conformance - Degree to which the knowledge representation model of the software product adheres to the knowledge representation model of an ontology language. Ontology language model conformance is a subcharacteristic of Functional Compliance and can be measured using:

- Ontology Language Component Support

- Ontology Language Component Coverage 
Ontology Processing Accuracy - Degree to which a product or system provides the correct results with the needed degree of precision when processing ontologies. Ontology Processing Accuracy is a sub-characteristic of Functional Correctness and can be measured using:

- Ontology Information Change

Ontology Processing Maturity - Degree to which a system, product or component meets needs for reliability when processing ontologies under normal operation. Ontology Processing Maturity is a sub-characteristic of Maturity and it can be measured using:

- Import/Export Errors

Ontology Language Model Interoperability - Degree to which the knowledge representation model of the software product adheres to the knowledge representation model of an ontology language after interchanging ontologies (importing and exporting an ontology using two different tools). Ontology language interoperability is a subcharacteristic of Interoperability and can be measured using:

- Ontology Language Component Interoperability Support

- Ontology Language Component Interoperability Coverage

Ontology Interchange Accuracy - Degree to which a product or system provides the correct results with the needed degree of precision when interchanging ontologies (importing and exporting an ontology using two different tools). Ontology Interchange Accuracy is the sub-characteristic of Ontology Processing Accuracy and can be measured using:

- Interchange Information Change

Ontology Interchange Maturity - Degree to which a system, product or component meets needs for reliability when interchanging ontologies (importing and exporting an ontology using two different tools) under normal operation. Ontology Interchange Maturity is a sub-characteristic of Ontology Processing Maturity and it can be measured using:

- Ontology Interchange Errors

Ontology Processing Time Behaviour - Degree to which the response, processing times and throughput rates of a product or system meet requirements when working with ontologies. Ontology Processing Time Behaviour is a sub-characteristic of Time Behaviour and can be measured using:

- Ontology Processing Time

Figure B.4 shows the part of the quality model obtained from the evaluation campaign for ontology engineering tools. 


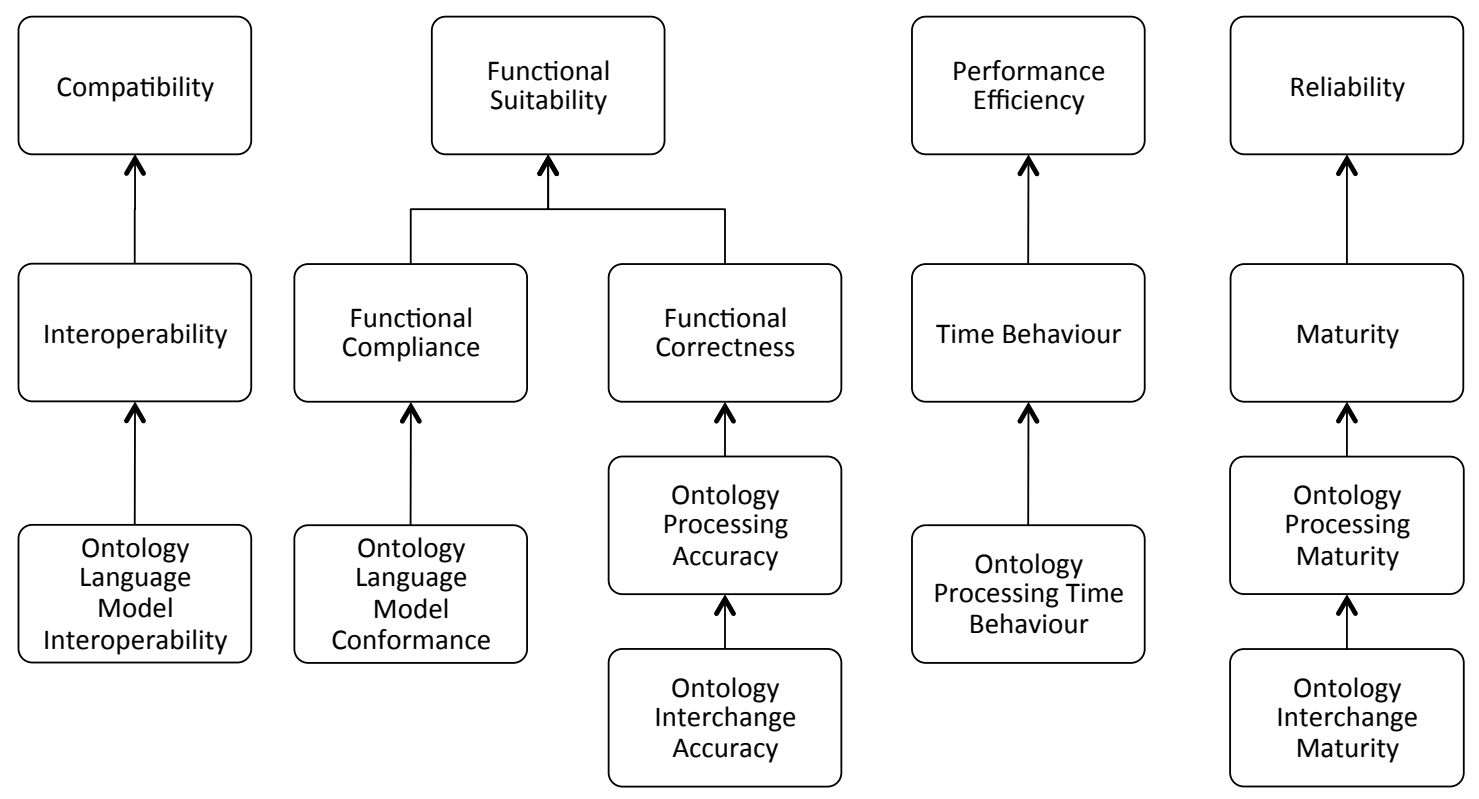

Figure B.4: Quality characteristics of ontology engineering tools.

\section{B.2 Reasoning Systems}

The evaluation campaign for reasoning systems contained evaluation scenarios to evaluate the accuracy, scalability, and maturity of these tools. Those scenarios include: classification scenario (Figure B.5), class satisfiability scenario (Figure B.6), ontology satisfiability scenario (Figure B.7), entailment scenario (Figure B.8), and non-entailment scenario (Figure B.9).

\section{B.2.1 Test Data}

\section{Classification Scenario}

Ontology - The ontology to be classified

\section{Class Satisfiability Scenario}

Ontology - The ontology to be used in a class satisfiability task

Class URIs - URIs of the classes to be checked for satisfiability

\section{Ontology Satisfiability Scenario}

Ontology - The ontology to be checked for satisfiability 


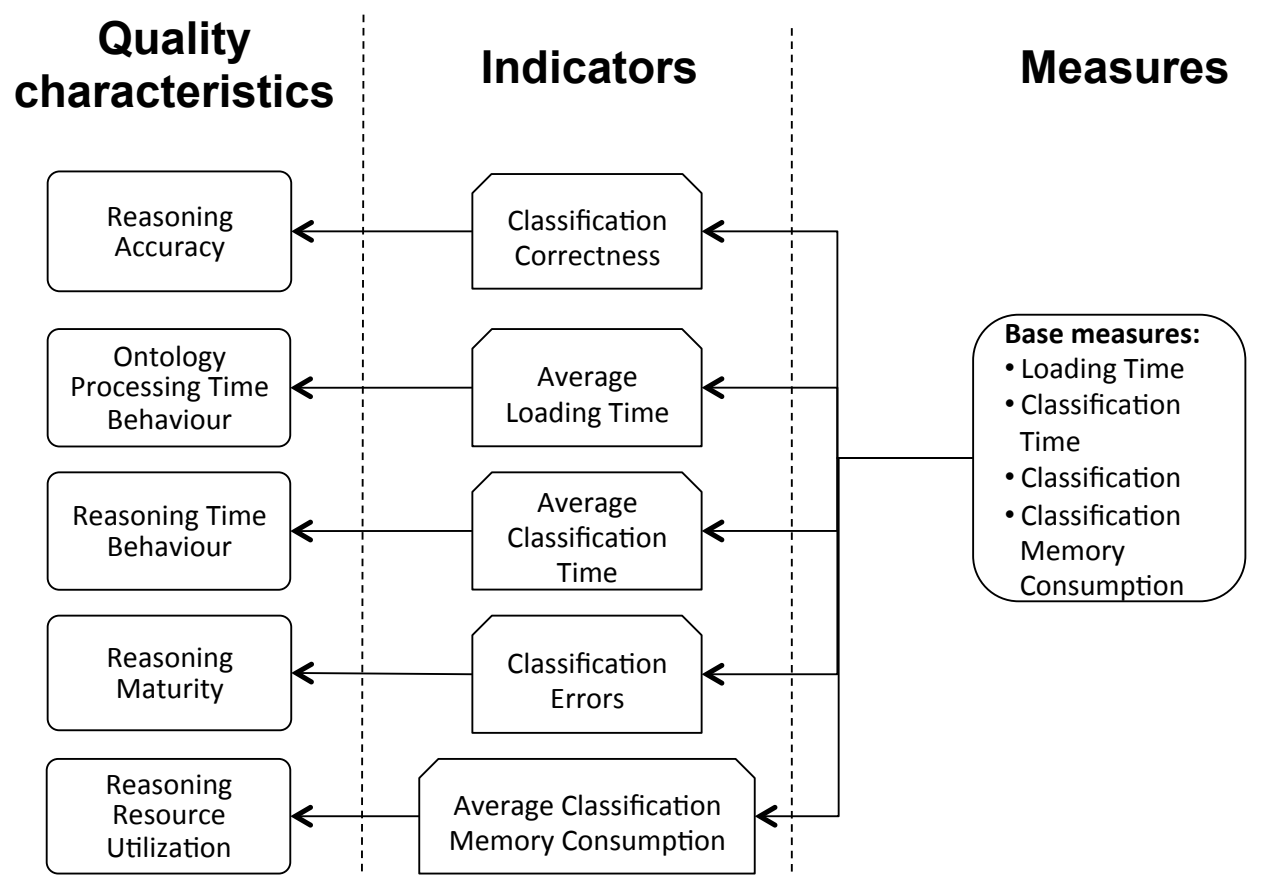

Figure B.5: Classification scenario of reasoning systems.

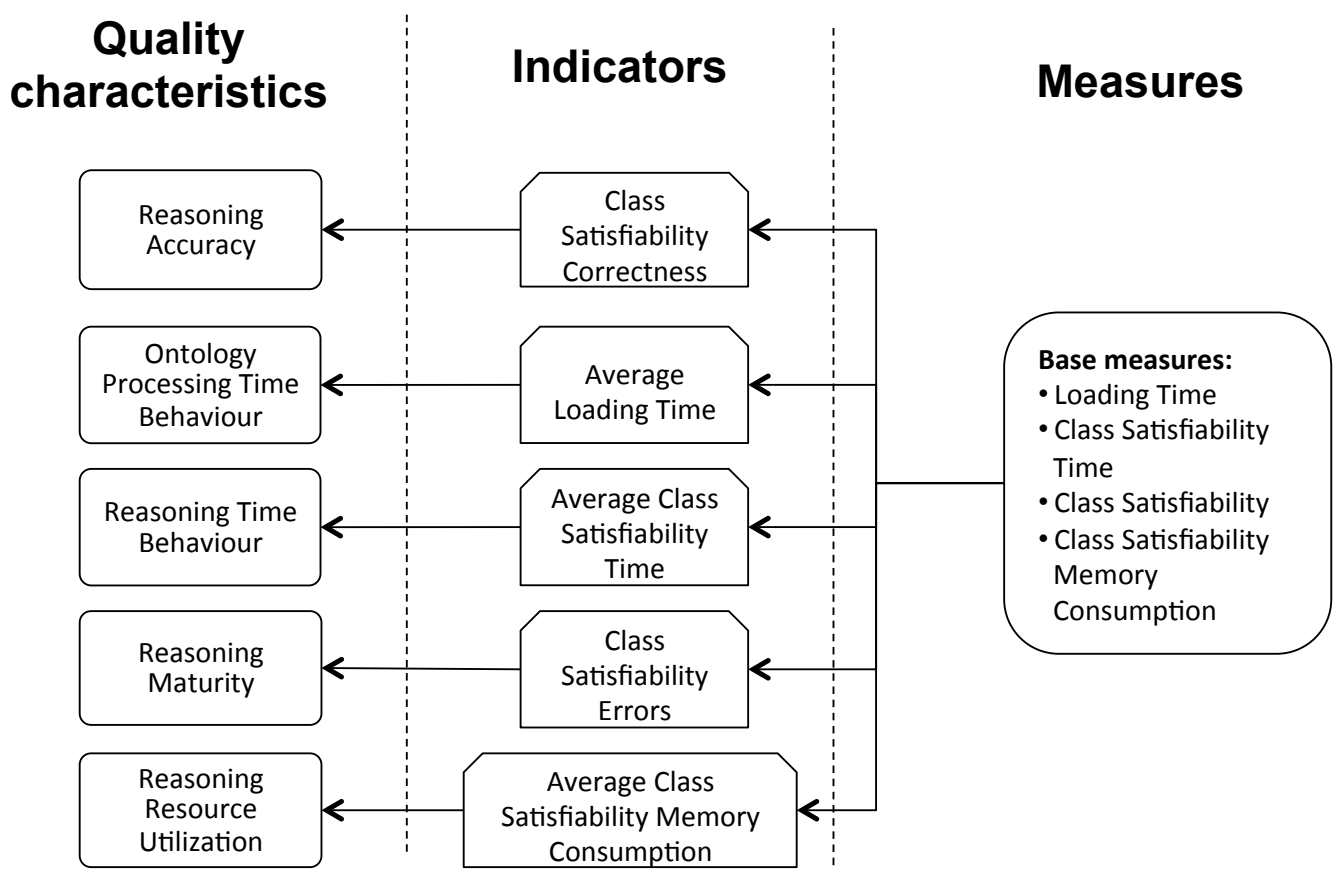

Figure B.6: Class satisfiability scenario of reasoning systems. 


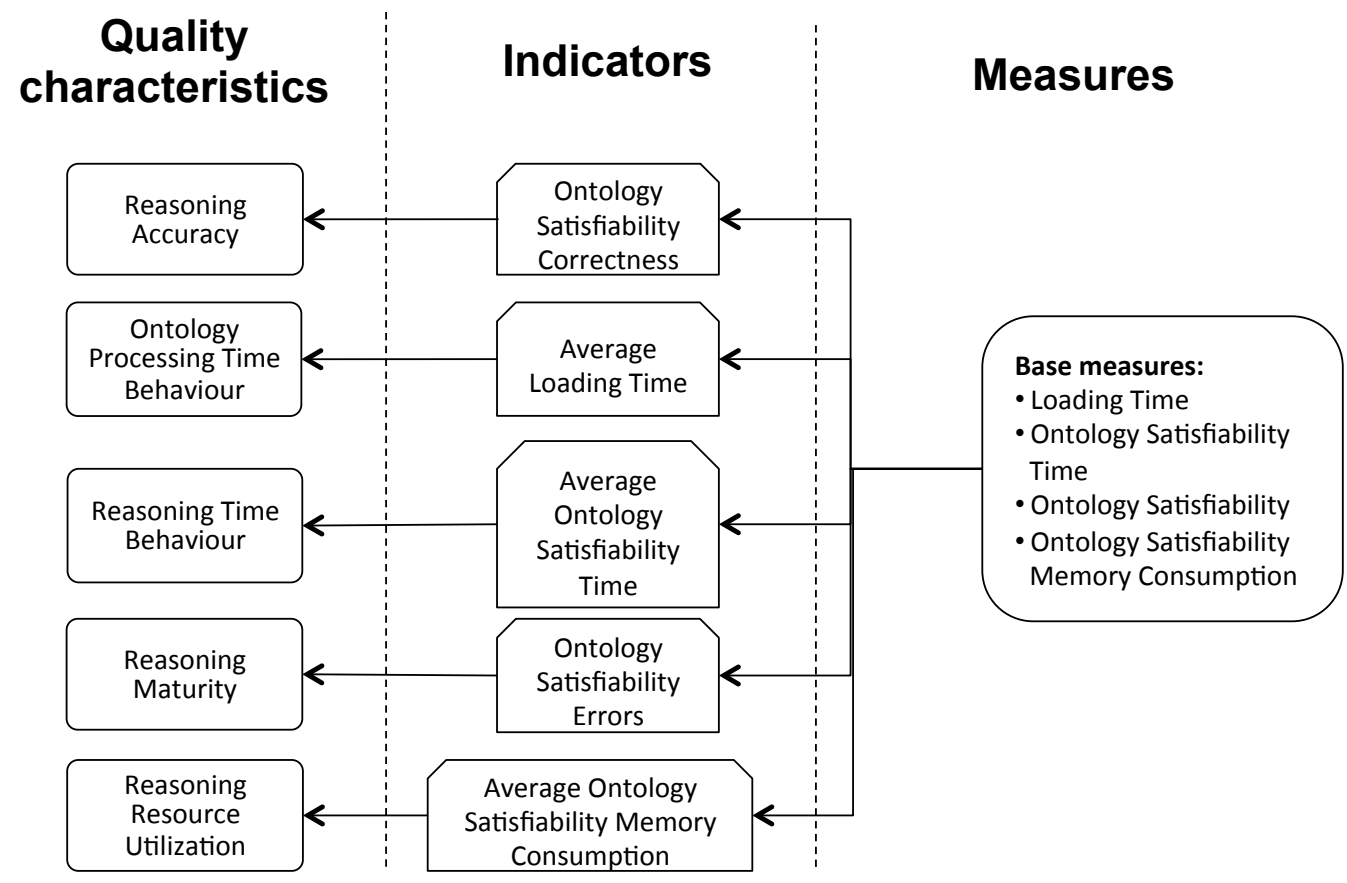

Figure B.7: Ontology satisfiability scenario of reasoning systems.

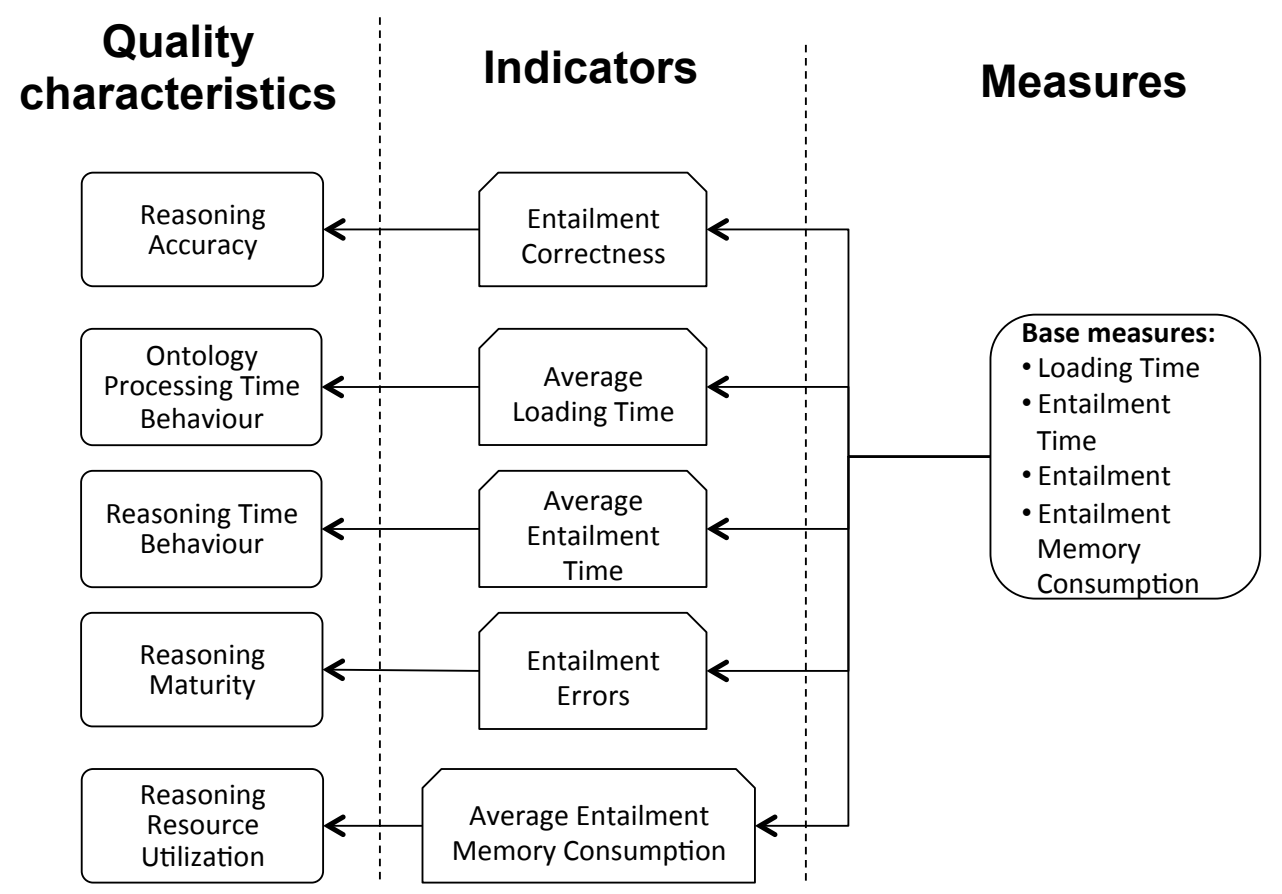

Figure B.8: Entailment scenario of reasoning systems. 


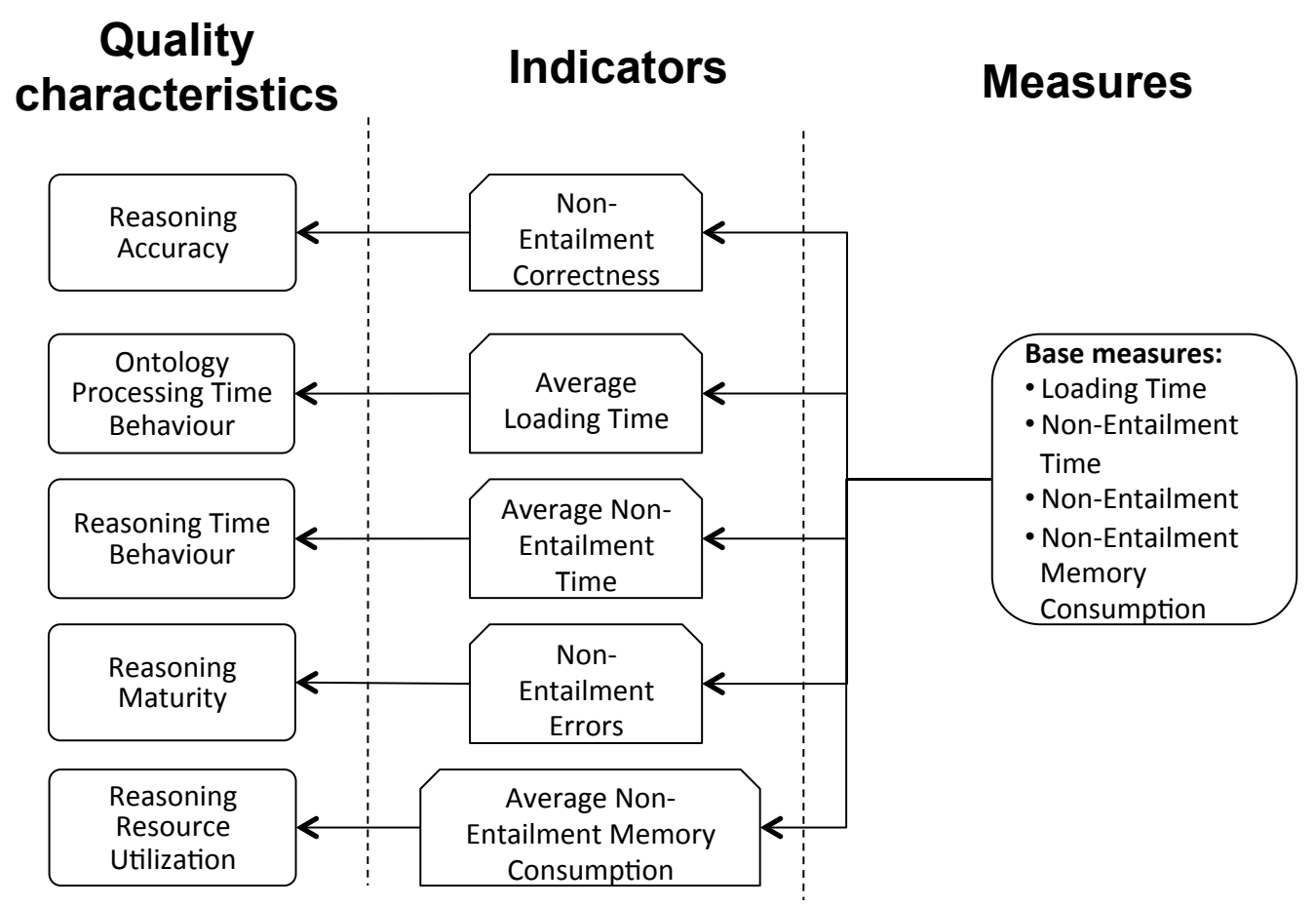

Figure B.9: Non-entailment scenario of reasoning systems.

\section{Entailment Scenario}

Premise Ontology - The ontology to be used in an entailment scenario Conclusion Ontology - The ontology to be checked for being logically entailed by the premise ontology

\section{Non-Entailment Scenario}

Premise Ontology - The ontology to be used in a non-entailment scenario Conclusion Ontology - The ontology to be checked for not being logically entailed by the premise ontology

\section{B.2.2 Base Measures}

\section{Common Base Measures}

Loading Time - The amount of time needed to load the ontology. Possible value is any number greater than 0 


\section{Classification Scenario}

Classification Time - The amount of time needed to perform a classification operation. Possible value is any number greater than 0

Classification - Whether the classification of the ontology is performed correctly. Possible values are true, false, unknown, and error

$$
\text { Classification }=\operatorname{classify}(\text { ontology })
$$

Classification Memory Consumption - The amount of memory consumed during a classification task. Possible value is any number greater than 0

\section{Class Satisfiability Scenario}

Class Satisfiability Time - The amount of time needed to perform a class satisfiability operation. Possible value is any number greater than 0

Class Satisfiability - Whether specific classes from the ontology are satisfiable. Possible values are true, false, unknown, and error

$$
\text { Class satisfiability }=\text { satisfiability (ontology, class URIs) }
$$

Class Satisfiability Memory Consumption - The amount of memory consumed during a class satisfiability task. Possible value is any number greater than 0

\section{Ontology Satisfiability Scenario}

Ontology Satisfiability Time - The amount of time needed to perform an ontology satisfiability operation. Possible value is any number greater than 0

Ontology Satisfiability - Whether the ontology is satisfiable. Possible values are true, false, unknown, and error

$$
\text { Ontology satisfiability }=\text { satisfiability (ontology) }
$$

Ontology Satisfiability Memory Consumption - The amount of memory consumed during an ontology satisfiability task. Possible value is any number greater than 0

\section{Entailment Scenario}

Entailment Time - The amount of time needed to perform entailment operation. Possible value is any number greater than 0 
Entailment - Whether the conclusion ontology is being logically entailed by the premise ontology. Possible values are true, false, unknown, and error

$$
\text { Entailment }=(\text { premise ontology } \mid=\text { conclusion ontology })
$$

Entailment Memory Consumption - The amount of memory consumed during an entailment task. Possible value is any number greater than 0

\section{Non-Entailment Scenario}

Non-Entailment Time - The amount of time needed to perform non-entailment operation. Possible value is any number greater than 0

Non-Entailment - Whether the conclusion ontology is not being logically entailed by the premise ontology. Possible values are true, false, unknown, and error

$$
\text { Non-entailment }=\neg(\text { premise ontology } \models \text { conclusion ontology })
$$

Non-Entailment Memory Consumption - The amount of memory consumed during a non-entailment task. Possible value is any number greater than 0

\section{B.2.3 Reasoning Systems Indicators}

\section{Common Indicators}

Average Loading Time - The average time needed for a tool to load an ontology. Possible value is any number greater than 0

$$
\text { Average loading time }=\frac{\sum_{n} \text { loading time for the } n^{\text {th }} \text { test }}{\# \text { tests }}
$$

\section{Classification Scenario}

Classification Correctness - The ratio of correctly performed classification tasks. Possible values are in range from 0 to 100

$$
\text { Classification correctness }=\frac{\# \text { tests } \mid(\text { classification }=\text { true })}{\# \text { tests }} \times 100
$$

Average Classification Time - The average time needed for a tool to perform a classification task. Possible value is any number greater than 0

$$
\text { Average classification time }=\frac{\sum_{n} \text { classification time for the } n^{\text {th }} \text { test }}{\# \text { tests }}
$$


Classification Errors - The ratio of tool execution errors when performing a classification task. Possible values are in range from 0 to 100

$$
\text { Classification errors }=\frac{\# \text { tests } \mid(\text { classification }=\text { error })}{\# \text { tests }} \times 100
$$

Average Classification Memory Consumption - The average amount of memory consumed during a classification task. Possible value is any number greater than 0

Average classification memory consumption $=$

$$
=\frac{\sum_{n} \text { classification memory consumption for the } n^{\text {th }} \text { test }}{\# \text { tests }}
$$

\section{Class Satisfiability Scenario}

Class Satisfiability Correctness - The ratio of correctly performed class satisfiability tasks. Possible values are in range from 0 to 100

$$
\text { Class satisfiability correctness }=\frac{\# \text { tests } \mid(\text { class satisfiability }=\text { true })}{\# \text { tests }} \times 100
$$

Average Class Satisfiability Time - The average time needed for a tool to perform a class satisfiability task. Possible value is any number greater than 0

$$
\text { Average class satisfiability time }=\frac{\sum_{n} \text { class satisfiability time for the } n^{\text {th }} \text { test }}{\# \text { tests }}
$$

Class Satisfiability Errors - The ratio of tool execution errors when performing a class satisfiability task. Possible values are in range from 0 to 100

$$
\text { Class satisfiability errors }=\frac{\# \text { tests where } \mid(\text { class satisfiability }=\text { error })}{\# \text { tests }} \times 100
$$

Average Class Satisfiability Memory Consumption - The average amount of memory consumed during a class satisfiability task. Possible value is any number greater than 0

Average class satisfiability memory consumption $=$

$$
=\frac{\sum_{n} \text { class satisfiability memory consumption for the } n^{\text {th }} \text { test }}{\# \text { tests }}
$$




\section{Ontology Satisfiability Scenario}

Ontology Satisfiability Correctness - The ratio of correctly performed ontology satisfiability tasks. Possible values are in range from 0 to 100

Ontology satisfiability correctness $=\frac{\# \text { tests } \mid(\text { ontology satisfiability }=\text { true })}{\# \text { tests }} \times 100$

Average Ontology Satisfiability Time - The average time needed for a tool to perform an ontology satisfiability task. Possible value is any number greater than 0

Average ontology satisfiability time $=\frac{\sum_{n} \text { ontology satisfiability time for the } n^{\text {th }} \text { test }}{\# \text { tests }}$

Ontology Satisfiability Errors - The ratio of tool execution errors when performing an ontology satisfiability task. Possible values are in range from 0 to 100

$$
\text { Ontology satisfiability errors }=\frac{\# \text { tests } \mid(\text { ontology satisfiability }=\text { error })}{\# \text { tests }} \times 100
$$

Average Ontology Satisfiability Memory Consumption - The average amount of memory consumed during an ontology satisfiability task. Possible value is any number greater than 0

Average ontology satisfiability memory consumption $=$

$$
=\frac{\sum_{n} \text { ontology satisfiability memory consumption for the } n^{\text {th }} \text { test }}{\# \text { tests }}
$$

\section{Entailment Scenario}

Entailment Correctness - The ratio of correctly performed entailment tasks. Possible values are in range from 0 to 100

$$
\text { Entailment correctness }=\frac{\# \text { tests } \mid(\text { entailment }=\text { true })}{\# \text { tests }} \times 100
$$

Average Entailment Time - The average time needed for a tool to perform an entailment task. Possible value is any number greater than 0

$$
\text { Average entailment time }=\frac{\sum_{n} \text { entailment time for the } n^{\text {th }} \text { test }}{\# \text { tests }}
$$

Entailment Errors - The ratio of tool execution errors when performing an entail- 
ment task. Possible values are in range from 0 to 100

$$
\text { Entailment errors }=\frac{\# \text { tests } \mid(\text { entailment }=\text { error })}{\# \text { tests }} \times 100
$$

Average Entailment Memory Consumption - The average amount of memory consumed during an entailment task. Possible value is any number greater than 0

Average entailment memory consumption $=$

$$
=\frac{\sum_{n} \text { entaiment memory consumption for the } n^{\text {th }} \text { test }}{\# \text { tests }}
$$

\section{Non-Entailment Scenario}

Non-Entailment Correctness - The ratio of correctly performed non-entailment tasks. Possible values are in range from 0 to 100

$$
\text { Non-entailment correctness }=\frac{\# \text { tests } \mid(\text { non-entailment }=\text { true })}{\# \text { tests }} \times 100
$$

Average Non-Entailment Time - The average time needed for a tool to perform a non-entailment task. Possible value is any number greater than 0

$$
\text { Average non-entailment time }=\frac{\sum_{n} \text { non-entailment time for the } n^{\text {th }} \text { test }}{\# \text { tests }}
$$

Non-Entailment Errors - The ratio of tool execution errors when performing a non-entailment task. Possible values are in range from 0 to 100

$$
\text { Non-entailment errors }=\frac{\# \text { tests } \mid(\text { non-entailment }=\text { error })}{\# \text { tests }} \times 100
$$

Average Non-Entailment Memory Consumption - The average amount of memory consumed during a non-entailment task. Possible value is any number greater than 0

Average non-entaiment memory consumption $=$

$$
=\frac{\sum_{n} \text { non-entailment memory consumption for the } n^{\text {th }} \text { test }}{\# \text { tests }}
$$




\section{B.2.4 SQuaRE Quality Characteristics}

A number of SQuaRE quality characteristics related to this case is already described in Section B.1.5. Here, only those SQuaRE quality characteristics that are new are listed.

Resource Utilization - Degree to which the amount and types of resources used by a product or system meet the requirements when performing its functions. Resource Utilization is a sub-characteristic of Performance Efficiency

\section{B.2.5 Reasoning Systems Quality Characteristics}

A number of semantic quality characteristics related to this case is already described in Section B.1.6. Here, only those semantic quality characteristics that are new are listed, together with already defined ones that have new quality indicators associated with it.

Reasoning Accuracy - Degree to which a product or system provides the correct results with the needed degree of precision when performing a reasoning task. Reasoning Accuracy is a sub-characteristic of Ontology Processing Accuracy and can be measured using:

- Classification Correctness

- Class Satisfiability Correctness

- Ontology Satisfiability Correctness

- Entailment Correctness

- Non-Entailment Correctness

Reasoning Time Behaviour - Degree to which the response, processing times and throughput rates of a product or system meet requirements when performing a reasoning task. Reasoning Time Behaviour is a sub-characteristic of Ontology Processing Time Behaviour and can be measured using:

- Average Classification Time

- Average Class Satisfiability Time

- Average Ontology Satisfiability Time

- Average Entailment Time

- Average Non-Entailment Time 
Reasoning Resource Utilization - Degree to which the amount and types of resources used by a product or system meet the requirements when performing a reasoning task. Reasoning Resource Utilization is a sub-characteristic of Resource Utilization and can be measured using:

- Average Classification Memory Consumption

- Average Class Satisfiability Memory Consumption

- Average Ontology Satisfiability Memory Consumption

- Average Entailment Memory Consumption

- Average Non-Entailment Memory Consumption

Reasoning Maturity - Degree to which a system, product or component meets needs for reliability when performing a reasoning task under normal operation. Reasoning Maturity is a sub-characteristic of Ontology Processing Maturity and it can be measured using:

- Classification Errors

- Class Satisfiability Errors

- Ontology Satisfiability Errors

- Entailment Errors

- Non-Entailment Errors

Ontology Processing Time Behaviour - Degree to which the response, processing times and throughput rates of a product or system meet requirements when working with ontologies. Ontology Processing Time Behaviour is a sub-characteristic of Time Behaviour and can be measured using:

- Average Loading time

Figure B.10 shows the part of the quality model obtained from the evaluation campaign for reasoning tools.

\section{B.3 Ontology Matching Tools}

The evaluation campaign for matching tools contained one evaluation scenario to evaluate accuracy (Figure B.11). 

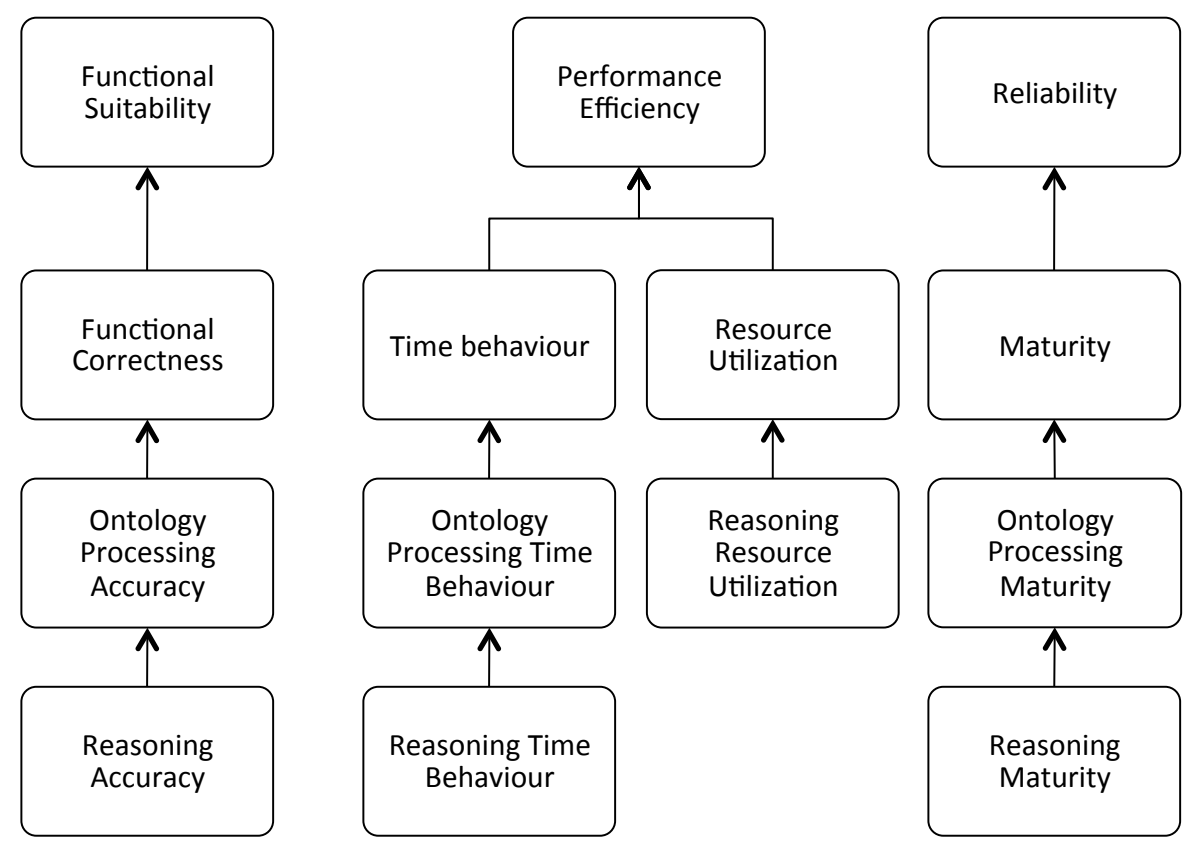

Figure B.10: Quality characteristics of reasoning systems.

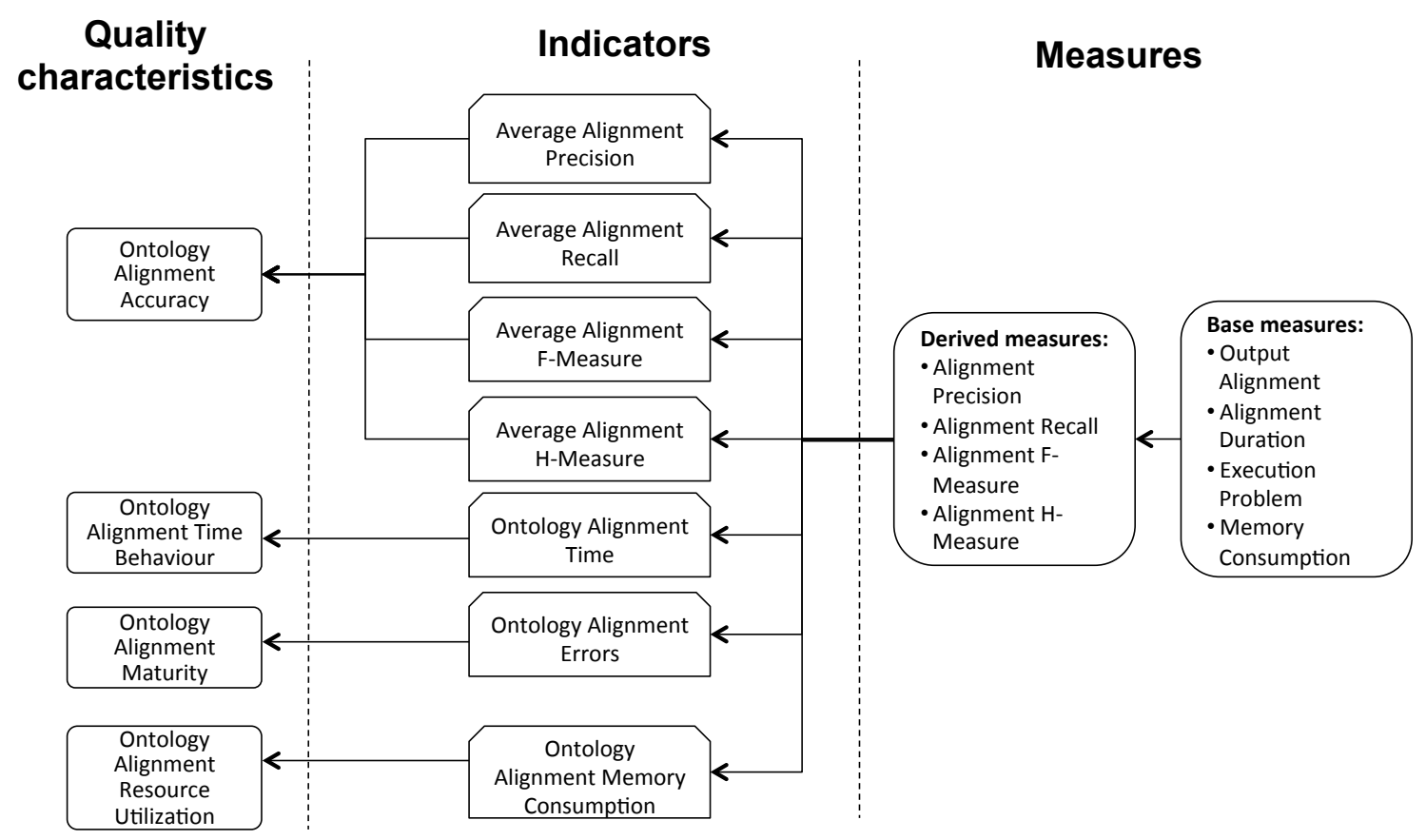

Figure B.11: Accuracy scenario of ontology matching tools. 


\section{B.3.1 Test Data}

Ontology 1 - One ontology to be used as input for an alignment task

Ontology 2 - Another ontology to be used as input for an alignment task

Expected Alignments - The expected alignments to be obtained when aligning the two input ontologies

\section{B.3.2 Base Measures}

Output Alignments - The alignments that are produced by the tool when aligning ontology 1 and ontology 2

$$
\text { Output alignment }=\operatorname{align}(\text { ontology } 1, \text { ontology } 2)
$$

Alignment Duration - The amount of time needed for an ontology alignment task. Possible value is any number greater than 0

Execution Problem - Whether there was any execution problem in the tool when performing an alignment task. Possible values are true, and false

Memory Consumption - The amount of memory consumed during an alignment task. Possible value is any number greater than 0

\section{B.3.3 Derived Measures}

Alignment Precision - The ratio between the output alignments returned by the tool that match expected alignments and all output alignments returned by the tool when performing an ontology matching task. Possible values are in range from 0 to 1

Alignment precision $=$

$$
=\frac{\# \text { output alignments } \mid \text { (output alignment } \in \text { expected alignments) }}{\text { \# output alignments }}
$$

Alignment Recall - The ratio between the output alignments returned by the tool that match expected alignments returned by the tool when performing an ontology matching task and the total number of expected alignments. Possible values are in range from 0 to 1

Alignment recall $=$

$$
=\frac{\# \text { output alignments } \mid \text { (output alignment } \in \text { expected alignments) }}{\# \text { expected alignments }}
$$

Alignment F-Measure - The aggregation measure of alignment precision and 
alignment recall. Possible values are in range from 0 to 1

Alignment F-measure $=$

$$
=\frac{\text { alignment precision } \times \text { alignment recall }}{(1-\alpha) \times \text { alignment precision }+\alpha \times \text { alignment recall }}, \alpha=[0 . .1]
$$

Alignment H-Measure - The aggregation measure of alignment precision and alignment recall (value of alignment F-Measure when $\alpha=0.5$ ). Possible values are in range from 0 to 1

$$
\text { Alignment H-measure }=\frac{\text { alignment precision } \times \text { alignment recall }}{\text { alignment precision }+ \text { alignment recall }} \times 2
$$

\section{B.3.4 Ontology Matching Tools Indicators}

Average Alignment Precision - The average precision of an ontology alignment task. Possible values are in range from 0 to 1

$$
\text { Average alignment precision }=\frac{\sum_{n} \text { alignment precision of the } n^{\text {th }} \text { test }}{\# \text { tests }}
$$

Average Alignment Recall - The average recall of an ontology alignment task. Possible values are in range from 0 to 1

$$
\text { Average alignment recall }=\frac{\sum_{n} \text { alignment recall of the } n^{\text {th }} \text { test }}{\# \text { tests }}
$$

Average Alignment F-Measure - The average F-Measure of an ontology alignment task. Possible values are in range from 0 to 1

$$
\text { Average alignment F-measure }=\frac{\sum_{n} \text { alignment F-Measure of the } n^{\text {th }} \text { test }}{\# \text { tests }}
$$

Average Alignment H-Measure - The average harmonic measure of an ontology alignment task. Possible values are in range from 0 to 1

$$
\text { Average alignment H-measure }=\frac{\sum_{n} \text { alignment H-measure of the } n^{\text {th }} \text { test }}{\# \text { tests }}
$$

Ontology Alignment Time - The average amount of time needed for an ontology alignment task. Possible value is any number greater than 0

$$
\text { Ontology alignment time }=\frac{\sum_{n} \text { alignment duration in the } n^{\text {th }} \text { test }}{\# \text { tests }}
$$


Ontology Alignment Errors - The ratio of tool execution errors when performing an ontology alignment task. Possible values are in range from 0 to 100

$$
\text { Ontology alignment errors }=\frac{\# \text { tests } \mid(\text { execution problem }=\text { true })}{\# \text { tests }} \times 100
$$

Ontology Alignment Memory Consumption - The average amount of memory consumed during an ontology alignment task. Possible value is any number greater than 0

Ontology alignment memory consumption $=$

$$
=\frac{\sum_{n} \text { memory consumption in the } n^{\text {th }} \text { test }}{\# \text { tests }}
$$

\section{B.3.5 SQuaRE Quality Characteristics}

All SQuaRE quality characteristics related to this case are already described in Section B.1.5 and Section B.2.4.

\section{B.3.6 Ontology Matching Tools Quality Characteristics}

A number of semantic quality characteristics related to this case is already described in Section B.1.6. Here, only those SQuaRE quality characteristics that are new are listed.

Ontology Alignment Accuracy - Degree to which a product or system provides the correct results with the needed degree of precision when performing an ontology alignment task. Ontology Alignment Accuracy is a sub-characteristic of Ontology Processing Accuracy and can be measured using:

- Average Alignment Precision

- Average Alignment Recall

- Average Alignment F-measure

- Average Alignment H-Measure

Ontology Alignment Maturity - Degree to which a system, product or component meets needs for reliability when performing an ontology alignment task under normal operation. Ontology Alignment Maturity is a sub-characteristic of Ontology Processing Maturity and can be measured using:

- Ontology Alignment Errors 
Ontology Alignment Time Behaviour - Degree to which the response, processing times and throughput rates of a product or system meet requirements when performing an ontology alignment task. Ontology Alignment Time Behaviour is a sub-characteristic of Ontology Processing Time Behaviour and can be measured using:

- Ontology Alignment Time

Ontology Alignment Resource Utilization - Degree to which the amount and types of resources used by a product or system meet the requirements when performing an ontology alignment task. Ontology Alignment Resource Utilization is a subcharacteristic of Resource Utilization and can be measured using:

- Ontology Alignment Memory Consumption

Figure B.12 shows the part of the quality model obtained from the evaluation campaign for matching tools.

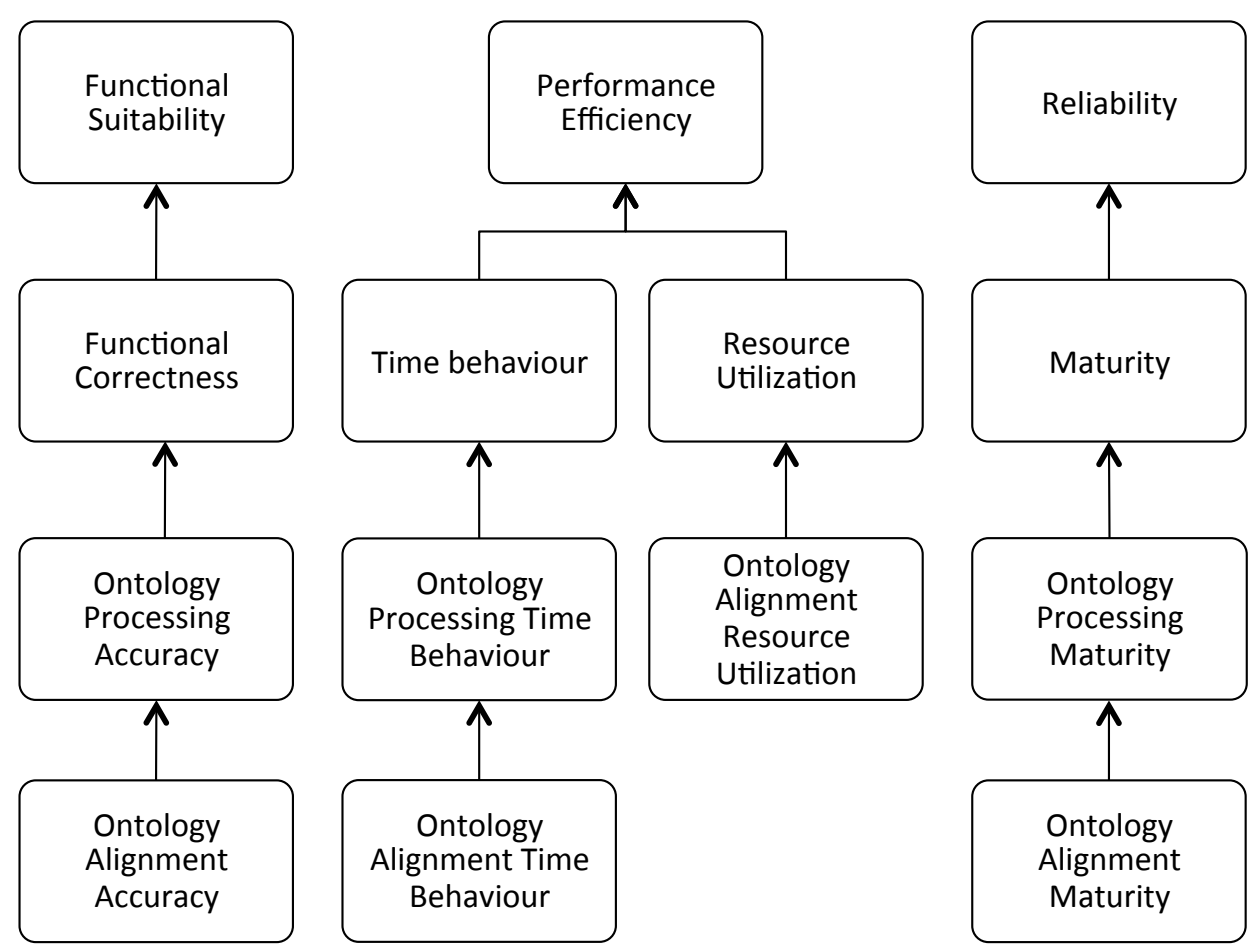

Figure B.12: Quality characteristics of ontology matching tools. 


\section{B.4 Semantic Search Tools}

The evaluation campaign for semantic search tools contained evaluation scenarios to evaluate accuracy, performance and usability. The evaluation was divided into two separate phases, an automated phase (Figure B.13 and Figure B.14) and a user-in-the-loop phase (Figure B.15 and Figure B.16).

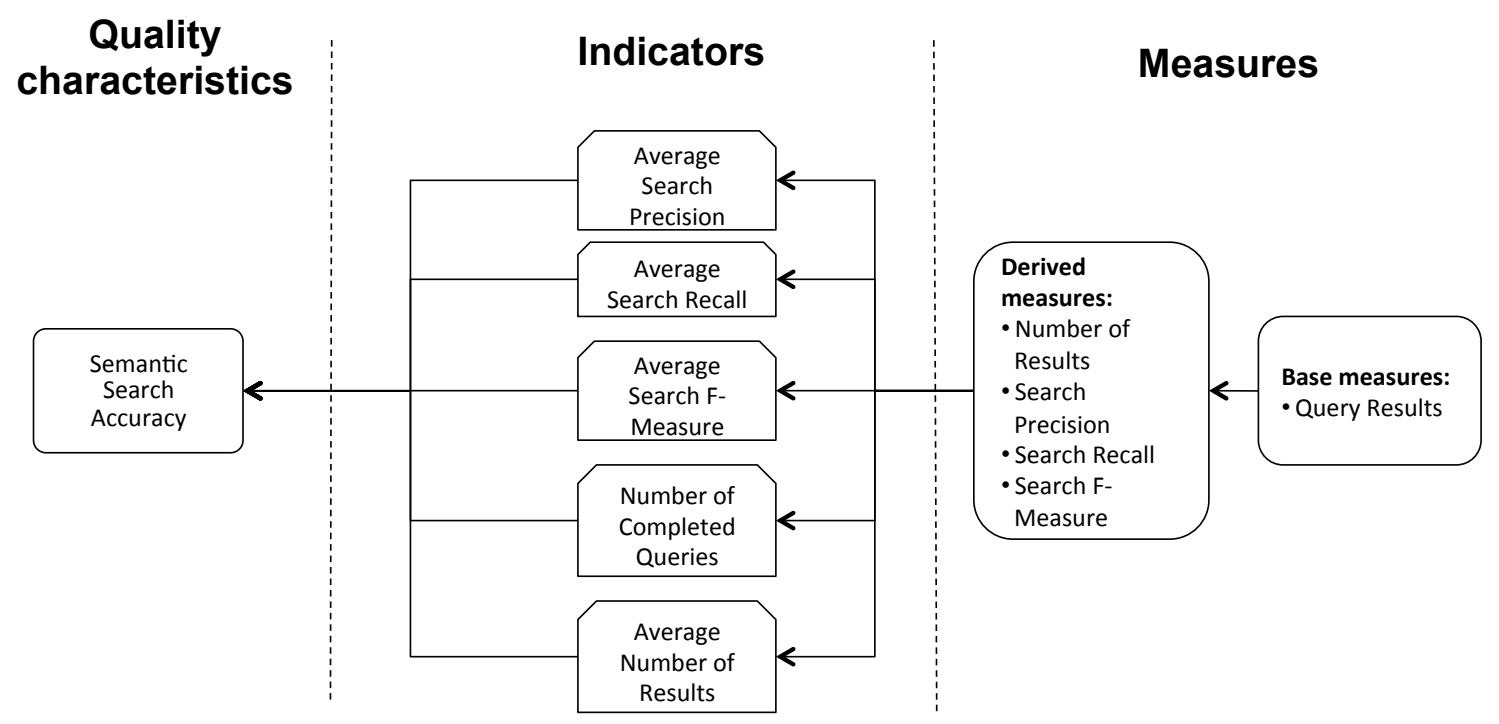

Figure B.13: Automated scenario (I) of semantic search tools.

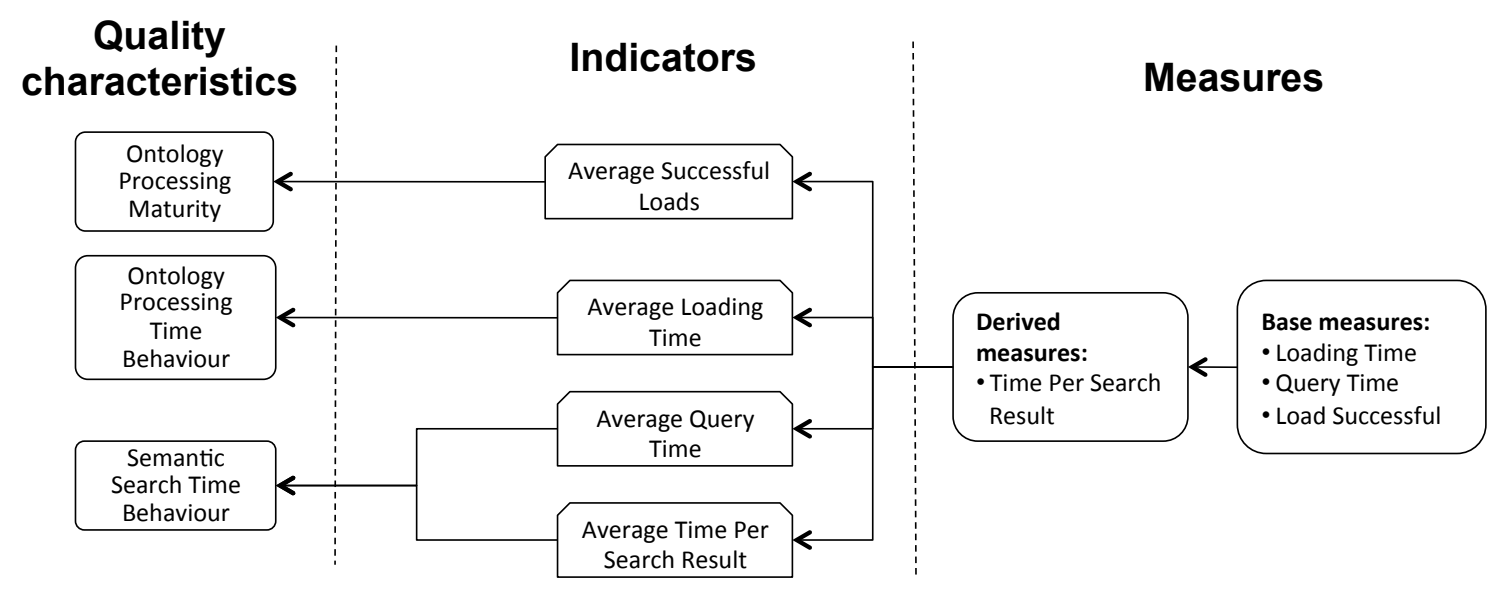

Figure B.14: Automated scenario (II) of semantic search tools. 


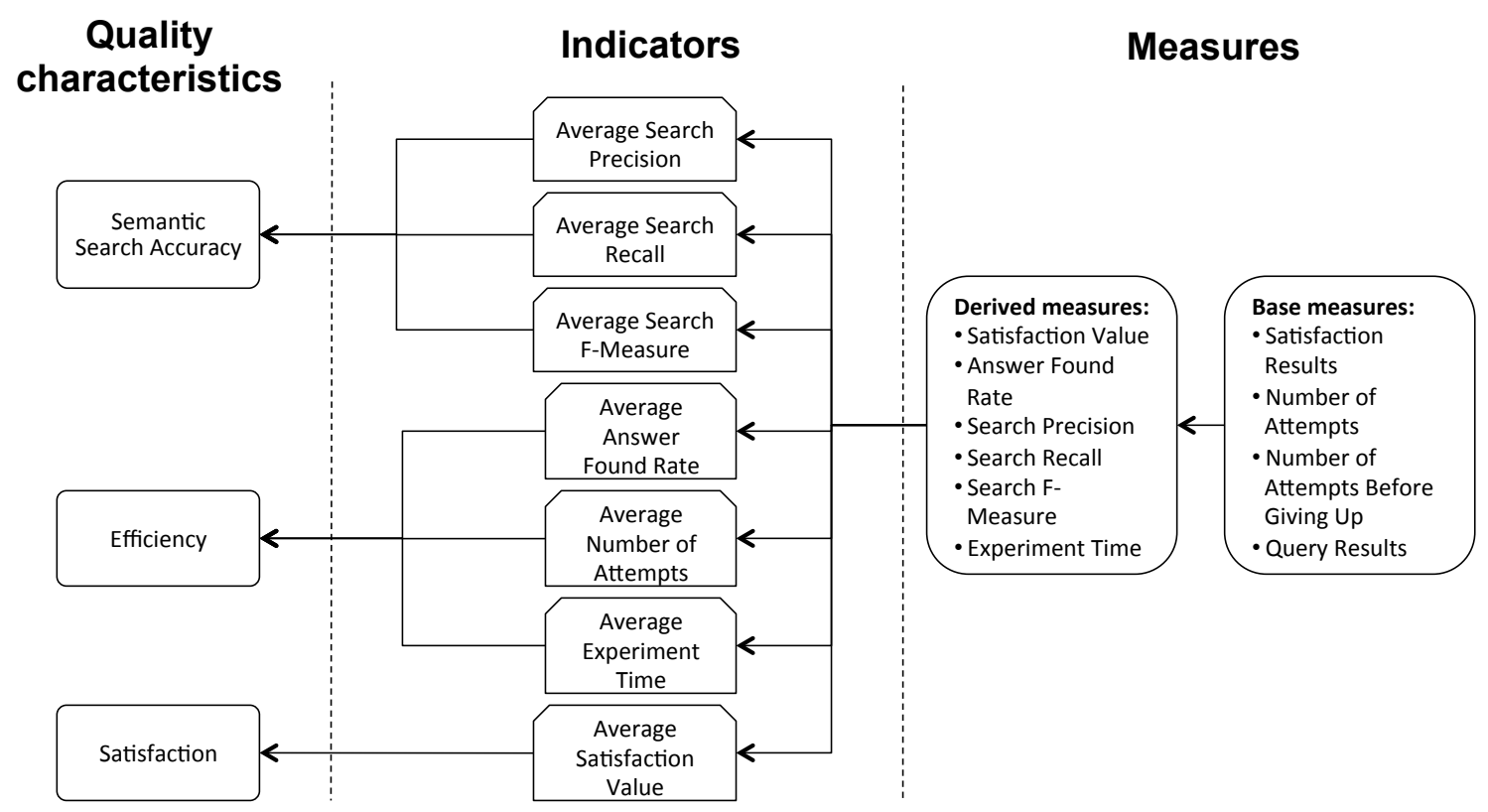

Figure B.15: User-in-the-loop scenario (I) of semantic search tools.

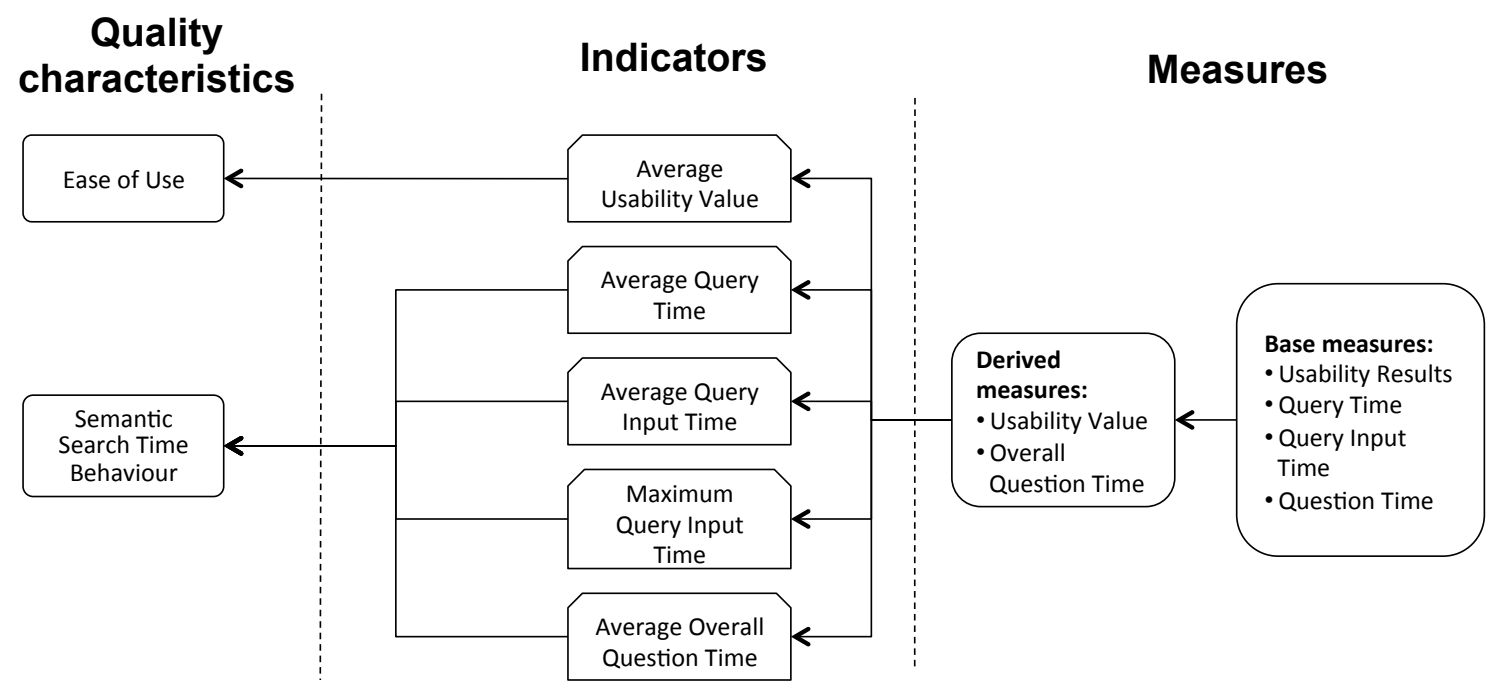

Figure B.16: User-in-the-loop scenario (II) of semantic search tools. 


\section{B.4.1 Test Data}

\section{Automated Scenario}

Origin Ontology - The ontology to be used as input for a search task

Input Question - The question to be used as input for a search task

Expected Answers - The expected answers to be obtained from the origin ontology for the input question

\section{User-In-The-Loop Scenario}

Origin Ontology - The ontology to be used as input for a search task

Input Question - The question to be used as input for a search task

Expected Answers - The expected answers to be obtained from the origin ontology for the input question

Usability Questionnaire - Standardized Usability Test by Brooke [17] containing ten standardized questions to be given to the users

Satisfaction Questionnaire - User satisfaction questionnaire to be given to the users

\section{B.4.2 Base Measures}

\section{Automated Scenario}

Query Results - The results returned by the tool when executing the query

$$
\text { Query results }=\operatorname{search}(\text { origin ontology, input question) }
$$

Loading Time - The amount of time needed to load the ontology. Possible value is any number greater than 0

Query Time - The amount of time needed to execute the query. Possible value is any number greater than 0

Load Successful - Whether the ontology has been successfully loaded. Possible values are true or false

\section{User-In-The-Loop Scenario}

Satisfaction Results - The results of the satisfaction questionnaire filled by the specific user

Number of Attempts - The number of times the user had to reformulate the query using the tool interface in order to obtain satisfactory answers for the input question. Possible value is any number greater than 0 
Number of Attempts Before Giving Up - The number of times the user had reformulated the query using the tool interface before giving up. Possible value is any number greater than 0

Query Results - The results returned by the tool when executing the query

Query results $=\operatorname{search}($ origin ontology, input question)

Usability Results - The results of the usability questionnaire filled by the specific user

Query Time - The amount of time needed to execute the query. Possible value is any number greater than 0

Query Input Time - The amount of time needed for the user to formulate a query using the tool's interface. Possible value is any number greater than 0

Question Time - The amount of time needed for one user to answer one question. Possible value is any number greater than 0

\section{B.4.3 Derived Measures}

\section{Automated Scenario}

Number of Results - The number of results obtained for the query. Possible value is any number greater or equal to 0

$$
\text { Number of results }=\text { count(query results) }
$$

Search Precision - The ratio between the relevant answers returned by the tool and all answers returned by the tool when performing a semantic search task. Possible values are in range from 0 to 1

$$
\text { Search precision }=\frac{\# \text { query results } \mid \text { (query result } \in \text { expected answers) }}{\# \text { query results }}
$$

Search Recall - The ratio between the relevant answers returned by the tool when performing a semantic search task and the total number of relevant answers. Possible values are in range from 0 to 1

$$
\text { Search recall }=\frac{\# \text { query results } \mid \text { (query result } \in \text { expected answers) }}{\# \text { expected answers }}
$$

Search F-Measure - The aggregation measure of semantic search precision and 
semantic search recall. Possible values are in range from 0 to 1

$$
\text { Search F-measure }=\frac{\text { search precision } \times \text { search recall }}{\text { search precision }+ \text { search recall }} \times 2
$$

Time Per Search Result - The amount of time needed to obtain one result. Possible value is any number greater than 0

$$
\text { Time per search result }=\frac{\text { query time }}{\text { number of results }}
$$

\section{User-In-The-Loop Scenario}

Satisfaction Value - The value obtained from the satisfaction questionnaire for a specific user. Possible values are in range from 0 to 100

$$
\text { Satisfaction value }=\text { interpret(satisfaction results) }
$$

Answer Found Rate - The ratio of finding the satisfactory answer for a specific question after a number of attempts and the users giving up after a number of attempts

$$
\text { Answer found rate }=\frac{\sum_{n} \text { number of attempts of the } n^{\text {th }} \text { user }}{\sum_{n} \text { number of attempts before giving up of the } n^{\text {th }} \text { user }}
$$

Search Precision - The ratio between the relevant answers and all answers returned by the tool when performing a semantic search task. Possible values are in range from 0 to 1

$$
\text { Search precision }=\frac{\# \text { query results } \mid \text { (query result } \in \text { expected answers) }}{\# \text { query results }}
$$

Search Recall - The ratio between the relevant answers returned by the tool when performing a semantic search task and the total number of relevant answers. Possible values are in range from 0 to 1

$$
\text { Search recall }=\frac{\# \text { query results } \mid \text { (query result } \in \text { expected answers })}{\# \text { expected answers }}
$$

Search F-Measure - The aggregation measure of semantic search precision and semantic search recall. Possible values are in range from 0 to 1

$$
\text { Search F-measure }=\frac{\text { search precision } \times \text { search recall }}{\text { search precision }+ \text { search recall }} \times 2
$$


Usability Value - The value obtained from the usability questionnaire for a specific user. Possible values are in range from 0 to 100

$$
\text { Usability value }=\text { interpret(usability results) }
$$

Experiment Time - The amount of time needed for a specific user to complete all input questions. Possible value is any number greater than 0

$$
\text { Experiment time }=\sum_{n} \text { question time of the } n^{\text {th }} \text { input question }
$$

Overall Question Time - The average time needed to answer a specific input question. Possible value is any number greater than 0

$$
\text { Overall question time }=\frac{\sum_{n} \text { question time of the } n^{\text {th }} \text { user }}{\# \text { users }}
$$

\section{B.4.4 Semantic Search Tools Indicators}

\section{Automated Scenario}

Average Search Precision - The average precision of a semantic search task. Possible values are in range from 0 to 1

$$
\text { Average search precision }=\frac{\sum_{n} \text { search precision of the } n^{\text {th }} \text { test }}{\# \text { tests }}
$$

Average Search Recall - The average recall of a semantic search task. Possible values are in range from 0 to 1

$$
\text { Average search recall }=\frac{\sum_{n} \text { search recall of the } n^{\text {th }} \text { test }}{\# \text { tests }}
$$

Average Search F-Measure - The average F-Measure of a semantic search task. Possible values are in range from 0 to 1

$$
\text { Average search F-measure }=\frac{\sum_{n} \text { search F-Measure of the } n^{\text {th }} \text { test }}{\# \text { tests }}
$$

Number of Completed Queries - The number of queries that produced expected answer. Possible value is any number greater or equal to 0

Number of completed queries $=\#$ tests $\mid$ (expected answers $\in$ query results) 
Average Number of Results - The average number of results obtained for a query. Possible value is any number greater or equal to 0

$$
\text { Average number of results }=\frac{\sum_{n} \text { number of results of the } n^{\text {th }} \text { test }}{\# \text { tests }}
$$

Average Successful Loads - The ratio of successful ontology loads. Possible values are in the range from 0 to 100

$$
\text { Average successful loads }=\frac{\# \text { tests } \mid(\text { load successful }=\text { true })}{\# \text { tests }} \times 100
$$

Average Loading Time - The average time needed for a tool to load an ontology. Possible value is any number greater than 0

$$
\text { Average loading time }=\frac{\sum_{n} \text { loading time of the } n^{\text {th }} \text { test }}{\# \text { tests }}
$$

Average Query Time - The average amount of needed to execute a query. Possible value is any number greater than 0

$$
\text { Average query time }=\frac{\sum_{n} \text { query time of the } n^{\text {th }} \text { test }}{\# \text { tests }}
$$

Average Time Per Search Result - The average amount of time needed to obtain one result. Possible value is any number greater than 0

$$
\text { Average time per search result }=\frac{\sum_{n} \text { time per search result of the } n^{\text {th }} \text { test }}{\# \text { tests }}
$$

\section{User-In-The-Loop Scenario}

Average Search Precision - The average precision of a semantic search task. Possible values are in range from 0 to 1

$$
\text { Average search precision }=\frac{\sum_{n} \text { search precision of the } n^{\text {th }} \text { test }}{\# \text { tests }}
$$

Average Search Recall - The average recall of a semantic search task. Possible values are in range from 0 to 1

$$
\text { Average search recall }=\frac{\sum_{n} \text { search recall of the } n^{\text {th }} \text { test }}{\# \text { tests }}
$$


Average Search F-Measure - The average F-Measure of a semantic search task. Possible values are in range from 0 to 1

$$
\text { Average search F-measure }=\frac{\sum_{n} \text { search F-Measure of the } n^{\text {th }} \text { test }}{\# \text { tests }}
$$

Average Answer Found Rate - The ratio of finding the appropriate answer after a number of attempts and the user giving up after a number of attempts for all questions. Possible value is any number greater than 0

$$
\text { Average answer found rate }=\frac{\sum_{n} \text { answer found rate of the } n^{\text {th }} \text { input question }}{\# \text { input questions }}
$$

Average Number of Attempts - The average number of attempts before the user is happy with the results. Possible value is any number greater than 0

$$
\text { Average number of attempts }=\frac{\sum_{n} \text { number of attempts of the } n^{\text {th }} \text { test }}{\# \text { tests }}
$$

Average Experiment Time - The average time needed for completing all input questions. Possible value is any number greater than 0

$$
\text { Average experiment time }=\frac{\sum_{n} \text { experiment time of the } n^{\text {th }} \text { user }}{\# \text { users }}
$$

Average Satisfaction Value - The average value obtained from the satisfaction questionnaires. Possible values are in range from 0 to 100

$$
\text { Average satisfaction value }=\frac{\sum_{n} \text { satisfaction value of the } n^{\text {th }} \text { user }}{\# \text { users }}
$$

Average Usability Value - The average value obtained from the usability questionnaires. Possible values are in range from 0 to 100

$$
\text { Average usability value }=\frac{\sum_{n} \text { usability value of the } n^{\text {th }} \text { user }}{\# \text { users }}
$$

Average Query Time - The average amount of needed to execute a query. Possible value is any number greater than 0

$$
\text { Average query time }=\frac{\sum_{n} \text { query time of the } n^{\text {th }} \text { test }}{\# \text { tests }}
$$


Average Query Input Time - The average time needed to formulate a query. Possible value is any number greater than 0

$$
\text { Average query input time }=\frac{\sum_{n} \text { query input time of the } n^{\text {th }} \text { test }}{\# \text { tests }}
$$

Maximum Query Input Time - The maximum time needed to formulate a query. Possible value is any number greater than 0

Maximum query input time $=$ maximum(query input time of the $n^{\text {th }}$ test)

Average Overall Question Time - The average amount of time needed to answer one question. Possible value is any number greater than 0

Average overall question time $=\frac{\sum_{n} \text { overall question time of the } n^{\text {th }} \text { input question }}{\# \text { input questions }}$

\section{B.4.5 SQuaRE Quality Characteristics}

A number of SQuaRE quality characteristics related to this case is already described in Section B.1.5. Here, only those SQuaRE quality characteristics that are new are listed.

Usability - Degree to which a product or system can be used by specified users to achieve specified goals with effectiveness, efficiency and satisfaction in a specified context of use

Operability - Degree to which a product or system has attributes that make it easy to operate and control. Operability is a sub-characteristic of Usability

Efficiency - Degree of resources expended in relation to the accuracy and completeness with which users achieve goals. Efficiency is a sub-characteristic of Quality in Use, and can be measured using:

- Average Number of Attempts

- Average Answer Found Rate

- Average Experiment Time

Satisfaction - Degree to which user needs are satisfied when a product or system is used in a specified context of use. Satisfaction is a sub-characteristic of Usability in Use, and can be measured using:

- Average Satisfaction Value 
Ease of Use - Degree to which the product or system makes it easy for users to operate and control it. Ease of Use is a sub-characteristic of Operability, and can be measured using:

- Average Usability Value

\section{B.4.6 Semantic Search Tools Quality Characteristics}

A number of semantic quality characteristics related to this case is already described in Section B.1.6. Here, only those semantic quality characteristics that are new are listed, together with already defined ones that have new quality indicators associated with it.

Semantic Search Accuracy - Degree to which a product or system provides the correct results with the needed degree of precision when performing a semantic search task. Semantic Search Accuracy is a sub-characteristic of Functional Correctness and can be measured using:

- Average Search Precision

- Average Search Recall

- Average Search F-measure

- Number of Completed Queries

- Average Number of Results

Semantic Search Time Behaviour - Degree to which the response, processing times and throughput rates of a product or system meet requirements when performing a semantic search task. Semantic Search Time Behaviour is a sub-characteristic of Time Behaviour and can be measured using:

- Average Query Time

- Average Time Per Search Result

- Average Execution Time

- Average Query Input Time

- Max Query Input Time

- Average Overall Question Time 
Ontology Processing Maturity - Degree to which a system, product or component meets needs for reliability when performing a semantic search task under normal operation. Ontology Processing Maturity is a sub-characteristic of Maturity and it can be measured using:

- Average Successful Loads

Ontology Processing Time Behaviour - Degree to which the response, processing times and throughput rates of a product or system meet requirements when working with ontologies. Ontology Processing Time Behaviour is a sub-characteristic of Time Behaviour and can be measured using:

- Average Loading Time

Figure B.17 shows the part of the quality model internal and external characteristics obtained from the evaluation campaign for semantic search tools, as well as quality in use.

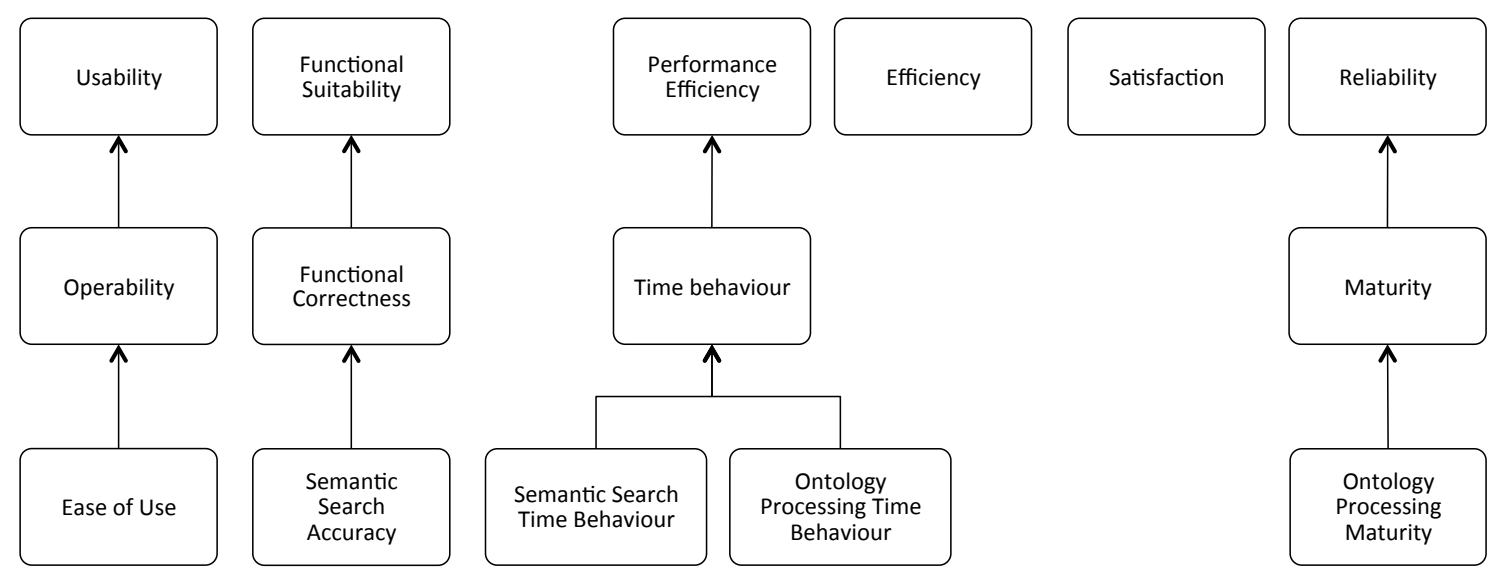

Figure B.17: Quality characteristics of semantic search tools.

\section{B.5 Semantic Web Service Tools}

The evaluation campaign for semantic web services contained one evaluation scenario to evaluate discovery (Figure B.18).

\section{B.5.1 Test Data}

Goal Document - The semantic web service document containing the users request description to be used in the discovery test 


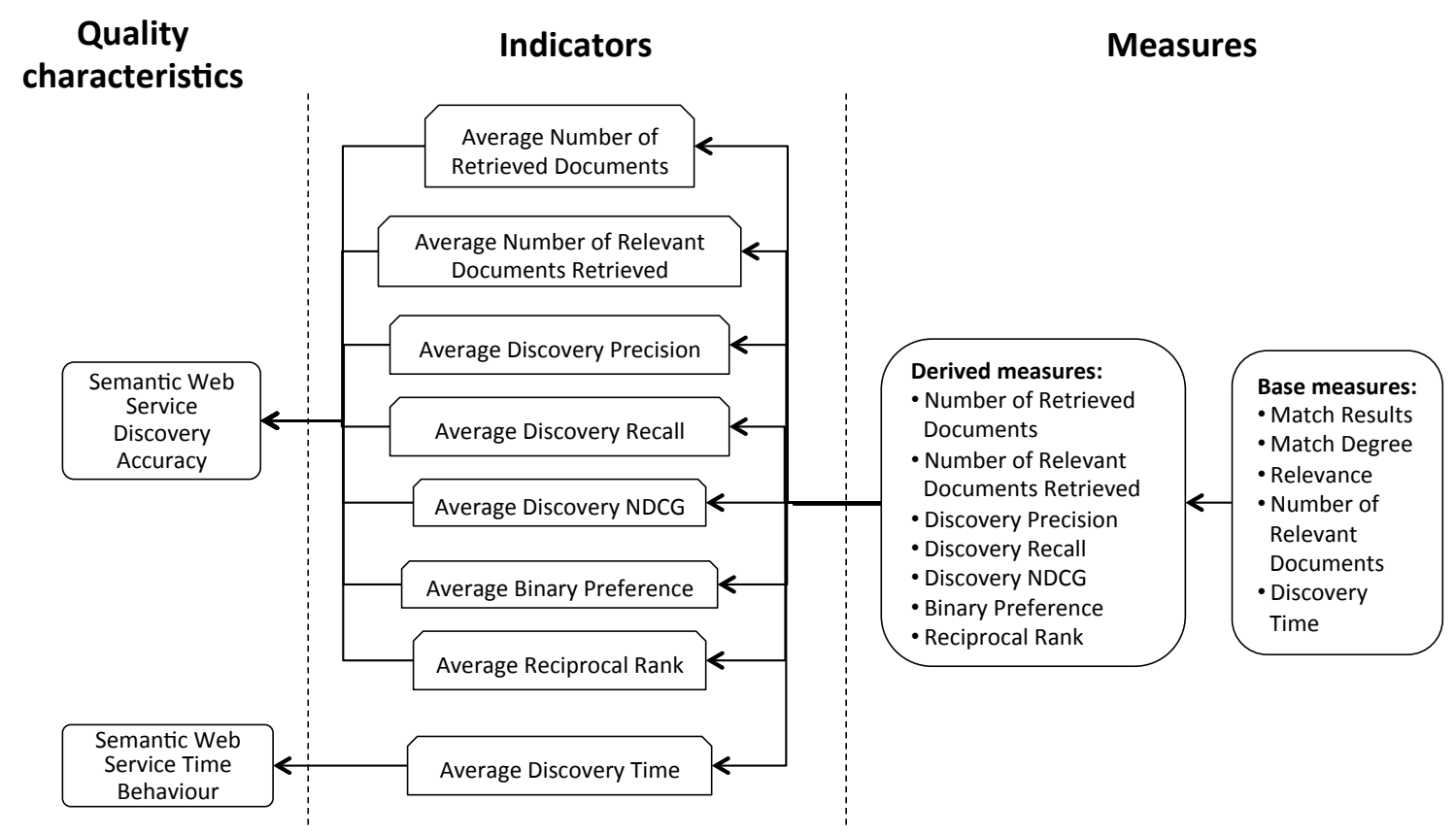

Figure B.18: Accuracy scenario of semantic web service tools.

Service Document - The semantic web service document containing all service offers to be used in the discovery test

Relevant Documents - A list of documents containing service offers relevant to the goal document and service document.

\section{B.5.2 Base Measures}

Match Results - The ranked list of documents containing service offers produced by the tool in the discovery task

$$
\text { Match results }=\text { discover (goal document, service document) }
$$

Match Degree - Match degree produced by the tool for each match result. Possible values are Non, Plugin, Exact, Subsumption

Relevance - The relevance of a result at particular position

$$
\text { Relevance }=\text { relevance }_{i}
$$

Number of Relevant Documents - The number of relevant documents. Possible 
value is any number greater than 0

Number of relevant documents $=$ count(relevant documents)

Discovery Time - The amount of time needed to perform the discovery task. Possible value is any number greater than 0

\section{B.5.3 Derived Measures}

Number of Retrieved Documents - The number of retrieved documents. Possible value is any number greater or equal to 0

Number of retrieved documents $\left(\mathrm{n}_{r}\right)=\operatorname{count}($ match results)

Number of Relevant Documents Retrieved - The number of retrieved documents that are relevant. Possible value is any number greater or equal to 0

$$
\begin{aligned}
& \text { Number of relevant documents retrieved }\left(\mathrm{n}_{\text {rel }}\right)= \\
& \quad \# \text { retrieved documents } \mid \text { (match degree }=\text { exact }) \wedge \\
& \text { (retrieved document } \in \text { relevant documents) }
\end{aligned}
$$

Discovery Precision - The ratio between the relevant documents retrieved by the tool and all documents retrieved by the tool when performing a semantic web service discovery task. Possible values are in range from 0 to 1

$$
\text { Discovery precision }=\frac{\text { number of relevant documents retrieved }}{\text { number of retrieved documents }}
$$

Discovery Recall - The ratio between the relevant documents retrieved by the tool when performing a semantic web service discovery task and the total number of relevant documents. Possible values are in range from 0 to 1

$$
\text { Discovery recall }=\frac{\text { number of relevant documents retrieved }}{\text { number of relevant documents }}
$$

Discovery Normalized Discounted Cumulative Gain (NDCG) - A normalized measure of the effectiveness of the discovery tool according to the relevance of tool's discoveries. Possible value is any number greater than 0

$$
\text { Discovery } \mathrm{NDCG}=\text { relevance }_{1}+\sum_{i=2}^{n_{r}} \frac{\text { relevance }_{i}}{\log _{2} i}
$$

Binary Preference - Measures the relevant documents retrieved $(r)$ when dealing 
with incomplete information

$$
\text { Binary preference }=\frac{1}{n_{r e l}} \sum_{r} 1-\frac{\mid \mathrm{n} \text { ranked higher than } \mathrm{r} \mid}{n_{r e l}},
$$

where $n$ is a member of the first $n_{\text {rel }}$ judged non relevant documents

Reciprocal Rank - Returns the reciprocal of the rank of the first relevant document retrieved, or zero if no relevant documents were retrieved. Possible values are in range from 0 to 1

$$
\text { Reciprocal rank }=\frac{1}{\text { rank of the first relevant document retrieved }}
$$

Based on these derived measures, other derived measures related to a particular given number of documents retrieved at cutoff-point or to a given number of relevant documents retrieved at cutoff-point can be calculated.

\section{B.5.4 Semantic Web Service Tools Indicators}

Average Number of Retrieved Documents - The average number of retrieved documents for all tests. Possible value is any number greater or equal to 0

Average number of retrieved documents $=$

$$
=\frac{\sum_{n} \text { number of retrieved documents of the } n^{\text {th }} \text { test }}{\# \text { tests }}
$$

Average Number of Relevant Documents Retrieved - The average number of retrieved documents that were judged relevant. Possible value is any number greater or equal to 0

Averagenumber of relevant documents retrieved $=$

$$
=\frac{\sum_{n} \text { number of retrieved relevant documents of the } n^{\text {th }} \text { test }}{\# \text { tests }}
$$

Average Discovery Precision - The average precision of a semantic web service discovery task. Possible values are in range from 0 to 1

Average discovery precision $=\frac{\sum_{n} \text { discovery precision of the } n^{\text {th }} \text { test }}{\# \text { tests }}$

Average Discovery Recall - The average recall of a semantic web service discovery 
task. Possible values are in range from 0 to 1

$$
\text { Average discovery recall }=\frac{\sum_{n} \text { discovery recall of the } n^{\text {th }} \text { test }}{\# \text { tests }}
$$

Average Discovery NDCG - The average NDCG of a semantic web service discovery task. Possible value is any number greater than 0

$$
\text { Average discovery NDCG }=\frac{\sum_{n} \text { discovery NDCG of the } n^{\text {th }} \text { test }}{\# \text { tests }}
$$

Average Binary Preference - The average binary preference of a semantic web service discovery task

$$
\text { Average binary preference }=\frac{\sum_{n} \text { binary preference of the } n^{\text {th }} \text { test }}{\# \text { tests }}
$$

Average Reciprocal Rank - The average reciprocal rank of a semantic web service discovery task. Possible values are in range from 0 to 1

$$
\text { Average reciprocal rank }=\frac{\sum_{n} \text { reciprocal rank of the } n^{\text {th }} \text { test }}{\# \text { tests }}
$$

Average Discovery Time - The average amount of time needed for a semantic web service discovery task. Possible value is any number greater than 0

$$
\text { Average discovery time }=\frac{\sum_{n} \text { discovery time of the } n^{\text {th }} \text { test }}{\# \text { tests }}
$$

Similar as in the case of derived measures, other indicators related to a particular given number of documents retrieved at cutoff-point or to a given number of relevant documents retrieved at cutoff-point can be calculated.

\section{B.5.5 SQuaRE Quality Characteristics}

All SQuaRE quality characteristics related to this case are already described in Section B.1.5.

\section{B.5.6 Semantic Web Service Tools Quality Characteristics}

Some semantic quality characteristics related to this case are already described in Section B.2.5. Here, only those SQuaRE quality characteristics that are new are listed. 
Semantic Web Service Discovery Accuracy - Degree to which a product or system provides the correct results with the needed degree of precision when finding services that can be used to fulfill a given requirement from the service requester. Semantic web service discovery accuracy is a sub-characteristic of Functional Correctness and can be measured using:

- Average Number Retrieved Documents

- Average Number of Relevant Documents Retrieved

- Average Precision

- Average Recall

- Average Discovery NDCG

- Average Binary Preference

- Average Reciprocal Rank

Furthermore, semantic web service discovery accuracy can be also measured using previous indicators calculated for a particular given number of documents retrieved at cutoff-point or for a given number of relevant documents retrieved at cutoff-point. Semantic Web Service Time Behaviour - Degree to which the response, processing times and throughput rates of a product or system meet requirements when performing a semantic web service discovery task. Semantic Web Service Time Behaviour is a sub-characteristic of Time Behaviour and can be measured using:

- Average Discovery Time

Figure B.19 shows the part of the quality model obtained from the evaluation campaign for semantic web services.

\section{B.6 Ontology Annotation Tools}

The evaluation scenario of ontology annotation tools contains one evaluation scenario to evaluate accuracy (Figure B.20).

\section{B.6.1 Test Data}

Input Document - A document to be annotated by the tool

User Annotations - Annotations of the input document provided by the users 


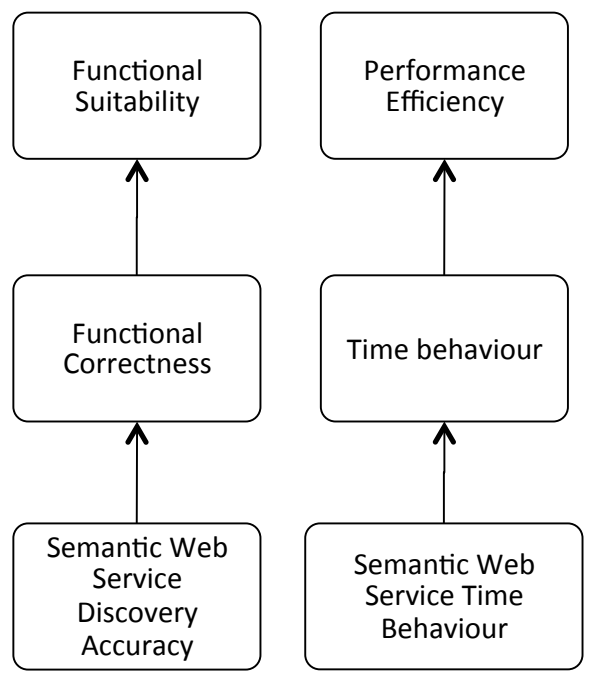

Figure B.19: Quality characteristics of semantic web service tools.

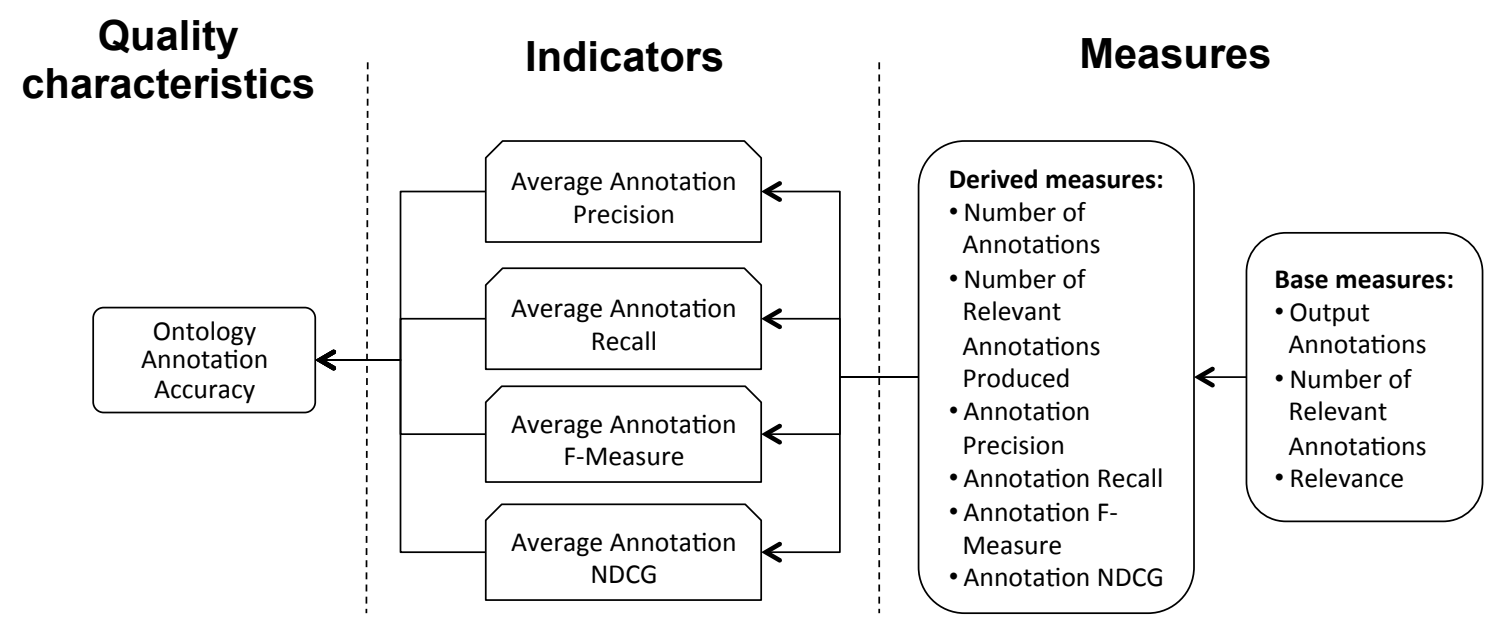

Figure B.20: Accuracy scenario of ontology annotation tools. 


\section{B.6.2 Base Measures}

Output Annotations - The annotations of the input document inferred by the tool

Output annotations $=$ annotate(input document)

Number of Relevant Annotations - The number of relevant annotations. Possible value is any number greater or equal to 0

Number of relevant annotations $=$ count(user annotations)

Relevance - The relevance of a particular annotation

$$
\text { Relevance }=\text { relevance }_{i}
$$

\section{B.6.3 Derived Measures}

Number of Annotations - The number of annotations produced by the tool. Possible value is any number greater or equal to 0

Number of annotations $\left(\mathrm{n}_{r}\right)=$ count(output annotations)

Number of Relevant Annotations Produced - Number of annotations produced by the tool that are relevant. Possible value is any number greater or equal to 0

Number of relevant annotations produced $=$

$$
=\# \text { output annotations } \mid \text { (output annotation } \in \text { user annotations) }
$$

Annotation Precision - The ratio between the relevant annotations produced by the tool and all annotations produced by the tool when performing an annotation task. Possible values are in range from 0 to 1

$$
\text { Annotation precision }=\frac{\text { number of relevant annotations produced }}{\text { number of annotations }}
$$

Annotation Recall - The ratio between the relevant annotations produced by the tool when performing an annotation task and the total number of relevant annotations. Possible values are in range from 0 to 1

$$
\text { Annotation recall }=\frac{\text { number of relevant annotations produced }}{\text { number of relevant annotations }}
$$

Annotation F-Measure - The aggregation measure of annotation precision and 
annotation recall. Possible values are in range from 0 to 1

Annotation F-measure $=$

$$
=\frac{\text { annotation precision } \times \text { annotation recall }}{(1-\alpha) \times \text { annotation precision }+\alpha \times \text { annotation recall }}, \alpha=[0 . .1]
$$

Annotation Normalized Discounted Cumulative Gain (NDCG) - A normalized measure of the effectiveness of the annotation tool according to the relevance of tool's annotations. Possible value is any number greater than 0

$$
\text { Annotation } \mathrm{NDCG}=\text { relevance }_{1}+\sum_{i=2}^{n_{r}} \frac{\text { relevance }_{i}}{\log _{2} i}
$$

\section{B.6.4 Ontology Annotation Tools Indicators}

Average Annotation Precision - The average precision of an ontology annotation task. Possible values are in range from 0 to 1

Average annotation precision $=\frac{\sum_{n} \text { annotation precision of the } n^{\text {th }} \text { test }}{\# \text { tests }}$

Average Annotation Recall - The average recall of an ontology annotation task. Possible values are in range from 0 to 1

$$
\text { Average annotation recall }=\frac{\sum_{n} \text { annotation recall of the } n^{\text {th }} \text { test }}{\# \text { tests }}
$$

Average Annotation F-Measure - The average F-Measure of an ontology annotation task. Possible values are in range from 0 to 1

$$
\text { Average annotation F-measure }=\frac{\sum_{n} \text { annotation F-Measure of the } n^{\text {th }} \text { test }}{\# \text { tests }}
$$

Average Annotation NDCG - The average NDCG of an ontology annotation task. Possible value is any number greater than 0

$$
\text { Average annotation NDCG }=\frac{\sum_{n} \text { annotation NDCG of the } n^{\text {th }} \text { test }}{\# \text { tests }}
$$

\section{B.6.5 SQuaRE Quality Characteristics}

All SQuaRE quality characteristics related to this case are already described in Section B.1.5. 


\section{B.6.6 Ontology Annotation Tools Quality Characteristics}

Ontology Annotation Accuracy - Degree to which a product or system provides the correct results with the needed degree of precision when performing an ontology annotation task. Ontology Annotation Accuracy is a sub-characteristic of Functional Correctness and can be measured using:

- Average Annotation Precision

- Average Annotation Recall

- Average Annotation F-measure

- Average Annotation NDCG

Figure B.21 shows the part of the quality model related to annotation tools.

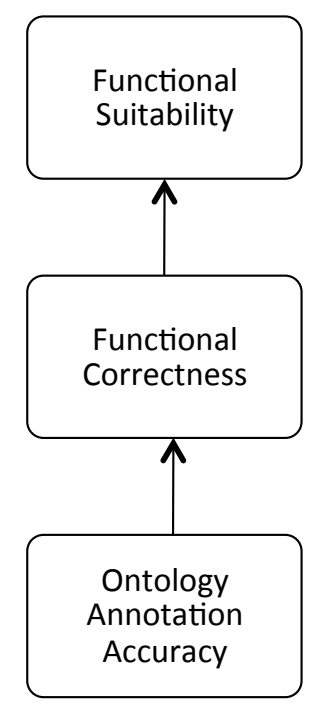

Figure B.21: Quality characteristics of ontology annotation tools.

\section{B.7 Complete Overview of the Quality Model}

Figure B.22 shows a complete overview of the quality model for semantic technologies that was obtained after analysing all the evaluation scenarios and results from the SEALS evaluation campaigns, and after the inclusion of the results of the SemQuaRE evaluation (Section 7.4). 


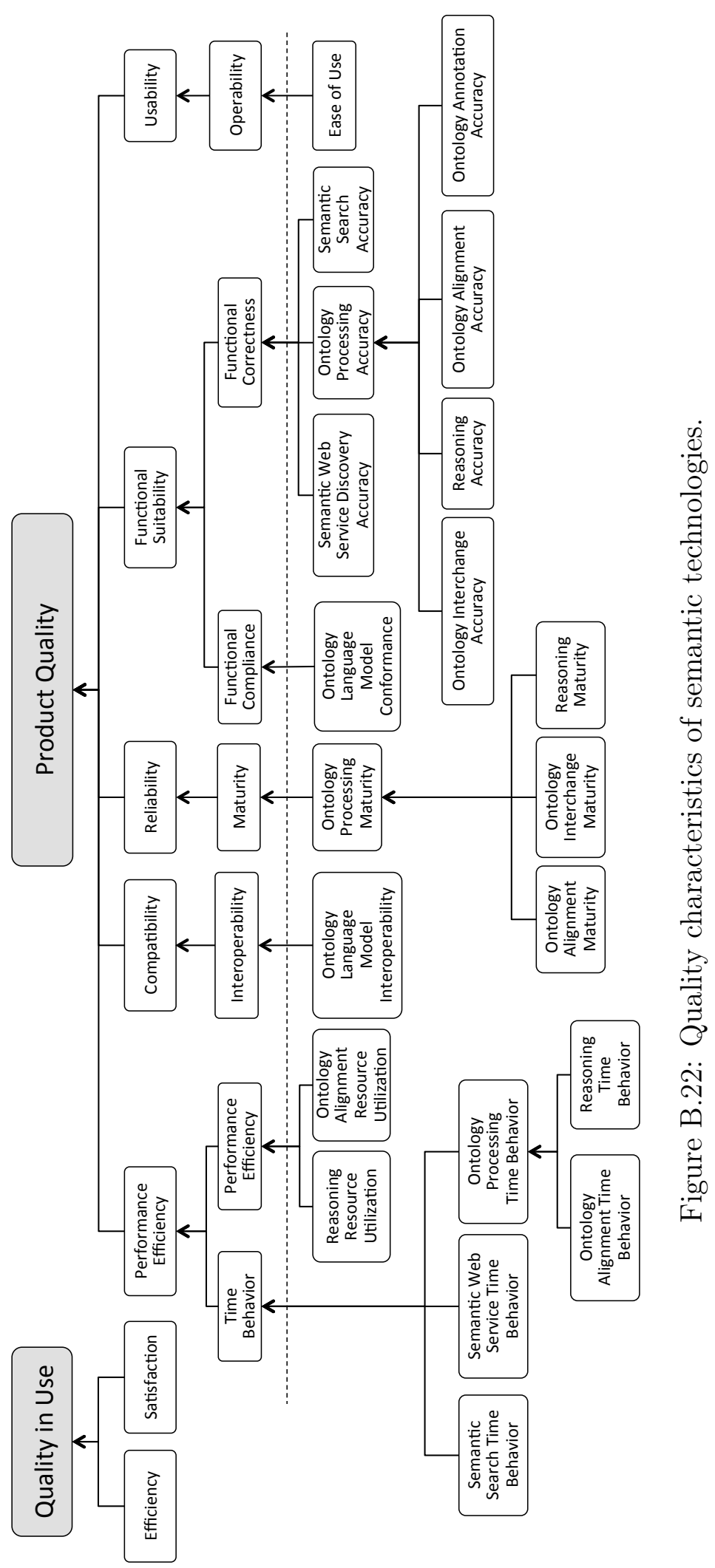

